\title{
Regional policies : convergence, trade, and the allocation of public capital
}

Citation for published version (APA):

Celbiş, M. G. (2015). Regional policies : convergence, trade, and the allocation of public capital. [Doctoral Thesis, Maastricht University]. Boekenplan. https://doi.org/10.26481/dis.20150625mc

Document status and date:

Published: 01/01/2015

DOI:

10.26481/dis.20150625mc

Document Version:

Publisher's PDF, also known as Version of record

\section{Please check the document version of this publication:}

- A submitted manuscript is the version of the article upon submission and before peer-review. There can be important differences between the submitted version and the official published version of record.

People interested in the research are advised to contact the author for the final version of the publication, or visit the DOI to the publisher's website.

- The final author version and the galley proof are versions of the publication after peer review.

- The final published version features the final layout of the paper including the volume, issue and page numbers.

Link to publication

\footnotetext{
General rights rights.

- You may freely distribute the URL identifying the publication in the public portal. please follow below link for the End User Agreement:

www.umlib.nl/taverne-license

Take down policy

If you believe that this document breaches copyright please contact us at:

repository@maastrichtuniversity.nl

providing details and we will investigate your claim.
}

Copyright and moral rights for the publications made accessible in the public portal are retained by the authors and/or other copyright owners and it is a condition of accessing publications that users recognise and abide by the legal requirements associated with these

- Users may download and print one copy of any publication from the public portal for the purpose of private study or research.

- You may not further distribute the material or use it for any profit-making activity or commercial gain

If the publication is distributed under the terms of Article $25 \mathrm{fa}$ of the Dutch Copyright Act, indicated by the "Taverne" license above, 


\section{Regional Policies}

Convergence, Trade, and the Allocation of Public Capital

Mehmet Güney Celbis 
ISBN 9789086663675

Copyright (c) Mehmet Güney Celbiş, 2015

All rights reserved. No part of this publication may be reproduced, stored in a retrieval system, or transmitted in any form, or by any means, electronic, mechanical, photocopying, recording or otherwise, without the prior permission in writing, from the author.

Publisher: Boekenplan, Maastricht 


\section{Regional Policies: Convergence, Trade, and the Allocation of Public Capital}

\section{DISSERTATION}

to obtain the degree of Doctor at Maastricht University, on the authority of Rector Magnificus, Prof. Dr. L.L.G. Soete, in accordance with the decision of the Board of Deans, to be defended in public on Thursday, 25 June 2015, at 12:00 hours

by

Mehmet Güney Celbiş 


\section{Supervisors}

Prof. Dr. Joan Muysken

Prof. Dr. Peter Nijkamp (VU University Amsterdam)

\section{Co-supervisor}

Dr. Denis de Crombrugghe

\section{Assessment Committee}

Prof. Dr. Franz Palm (Chair)

Prof. Dr. Jouke van Dijk (University of Groningen)

Prof. Dr. Pierre Mohnen

Prof. Dr. Bert van Wee (Delft University of Technology) 


\section{Acknowledgements}

There are many people I would like to thank. My family and friends have been the greatest support for me during this undertaking. I am very grateful to my supervisors Joan Muysken, Peter Nijkamp, and Denis de Crombrugghe, and to Jacques Poot - who was my "unofficial supervisor" - for their invaluable guidance.

I am very grateful to Mindel van de Laar, Fransizka Gassman, and Chris De Neubourg for admitting me to the $\mathrm{PhD}$ programme which allowed me to embark on this quest at the first place. I would like to thank Lutz Krebs for counting on me and helping me to earn vaulable experience in academia during my $\mathrm{PhD}$.

At UNU-MERIT/MGSoG, I have been in an extremely friendly environment and received a lot of support by both current and former members. In this regard, I would like to thank Eveline in de Braek, Ad Notten, Howard Hudson, Danny Beckers, Herman Pijpers, Susan Roggen, Janneke Knaapen, Eric Engelen, Sueli Brodin, Pui-hang Wong, Sachin Kumar Badkas, Serdar Turkeli, Richard Bluhm, Mahmut Kobal, Cheng Boon Ong, Nevena Zhelyazkova, Denisa Maria Sologon, Victor Cebotari, Manoj Kumar Dora, Katherine Kuschminder, Sonja Fransen, Silja Weyel, Ilire Agimi, Özge Bilgili, Irina Burlacu, Carlos Cadena Gaitan, Luciana Cingolani, Kristine Farla, Jojo Jacob, Zina Nimeh, Tatiana Skripka, Melissa Siegel, Florian Henning, Sonila Tomini, Florian Tomini, Andrea Franco-Correa, Paula Nagler, Daniel Opolot, Tatevik Poghosyan, Iman Rajabzadeh, Omar Rodriguez, Shuan SadreGhazi, Patricia Silva, Michiko Iizuka, Ayokunu Adedokun, Eleni Abraham Yitbarek, Yesuf Awel, Ibrahima Sory Kaba, Tobias Broich, Dorcas Mbuvi, Saba Afeworki, 
Samyukta Bhupatiraju, Alejandro Lavopa, Sepideh Yousefzadeh, Martin Rehm, and Margaret Rugadya.

I would also like to thank Henry Chappell, McKinley Blackburn, John McDermott, Janice Boucher Breuer, Paul Boyd, Scott Ranges, Cem Özgüzel, Daniela Dora, Selburn Christian, Srideep Ganguly, Faruk Bülbül, Nazmi Demir, Güçlü Özkök, and Masood Gheasi. 


\section{Contents}

1 Introduction 1

1.1 Background and Relevance . . . . . . . . . . . 1

1.2 Research Outline . . . . . . . . . . . . . . 7

1.2.1 Internet infrastructure and regional disparities (chapter 2$) \ldots \ldots \ldots \ldots \ldots \ldots$

1.2.2 A meta-analysis on infrastructure and trade (chapter 3$) \ldots \ldots \ldots \ldots$

1.2.3 Regional exports and infrastructure in Turkey (chapter 4$) \ldots \ldots \ldots \ldots \ldots$

1.2.4 Political influences (chapter 5) . . . . . . . 13

1.3 Generalizability and Policy Significance . . . . . . . 14

\section{Can Internet Infrastructure Help Reduce Regional} Disparities? Evidence from Turkey

2.1 Introduction . . . . . . . . . . . . . . 17

2.2 Theoretical Background . . . . . . . . . . . 20

2.2.1 The theory and analysis of convergence . . . 20 
2.2.2 $\sigma$-convergence and its relation to $\beta$-convergence 22

2.2.3 Communications, geography, and convergence . 24

2.3 Regional Patterns of Income Per Capita in Turkey . . 27

2.4 Empirical Approach ............... 36

2.5 Data................... . . . 41

2.6 Estimation Results ............... 42

2.6.1 Cross-sectional estimation ......... 42

2.6.2 Panel estimation ............. . . 44

2.7 Concluding Remarks ............... 52

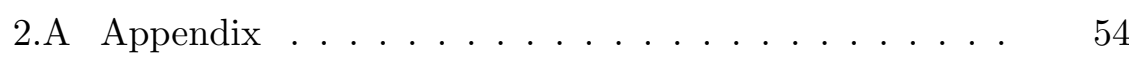

3 Infrastructure and Trade: A Meta-Analysis 59

3.1 Introduction ................. . . 59

3.2 Literature Review ............... . . 62

3.3 The Theory of Modeling Trade Flows . . . . . . . . 65

3.4 Methodology .................. 69

3.5 Data ................... . . . 71

3.6 Descriptive Analysis . . . . . . . . . . . . . . 79

3.7 Meta-regression Models .............. 86 
3.7 .1 Methodology . . . . . . . . . . . . . 92

3.7.2 The point at which the trade is measured . . . 93

$3.7 .3 \quad$ Infrastructure category . . . . . . . . . . . 95

3.7.4 Development level of the economy in which the infrastructure is located . . . . . . . . . 95

3.7.5 Sample structure . . . . . . . . . . . 95

3.7.6 Model specification . . . . . . . . . . . . 96

3.7.7 Nature of publication . . . . . . . . 97

3.7.8 Model prediction . . . . . . . . . . . . . 97

3.8 Concluding Remarks . . . . . . . . . . . . . . . . . 103

3.A Appendix .................... 106

4 Infrastructure and the International Export Performance of Turkish Regions

109

4.1 Introduction . . . . . . . . . . . . . . . 109

4.2 Theoretical Framework . . . . . . . . . . . . . . . . . 111

4.3 Data and Descriptive Statistics . . . . . . . . . 114

4.4 Estimation and Empirical Results . . . . . . . . . . 127

4.5 Concluding Remarks . . . . . . . . . . . . . 135

4.A Appendix .................... 137 
4.A.1 Additional estimation results . . . . . . . 137

4.A.2 Conversion to constant prices ......... 140

4.A.3 Land infrastructure index construction . . . . . 140

4.A.4 Exchange rates ............. 141

4.A.5 DSL data imputation . . . . . . . . . 141

5 Public Investment and Regional Politics: The Case of Turkey

5.1 Introduction . . . . . . . . . . . . . . 145

5.2 Research Motivation and Contribution . . . . . . . 147

5.2.1 Public capital and regional goals . . . . . . 147

5.2.2 Political influences and investment allocation . 149

5.2.3 Regional public infrastructure in Turkey . . . . 152

5.3 Theoretical Framework and Empirical Approach . . . 154

5.4 Data and Descriptive Statistics . . . . . . . . 160

5.4.1 Presenting a modified output and public investment series for Turkish regions . . . . . . 160

5.4.2 Infrastructure stock data . . . . . . . . 162

5.5 Empirical Results ................. 164

5.6 Concluding Discussion and Policy Implications . . . . 169 
5.A Appendix .................... 170

5.A.1 Gross Value Added Data Adjustments . . . . 170

5.A.2 Public Investments Data Adjustments . . . . 170

6 Conclusion

185

6.1 A Review of the Research Findings and Their Implications . . . . . . . . . . . . . . . 185

6.2 Remedies Provided for the Problems in Turkish Regional Data Sets . . . . . . . . . . . . . 189

6.3 Methodological Approaches, Challenges and Their Implications . . . . . . . . . . . . . . . . 191

6.4 Future Research Prospects . . . . . . . . . . . . . . 192 


\section{List of Tables}

2.1 Descriptive statistics . . . . . . . . . .

2.2 Cross-sectional estimation results . . . . . . . . 48

2.3 Panel estimation results . . . . . . . . . . . 49

2.4 Panel estimation results ............. 50

2.5 Convergence factors, speeds, and associated half-lives (Base model) ................ . . 51

2.6 Model comparison versus GSM ............ 51

2.A.1 Examples of variables used in the analysis of convergence................. . . . 54

2.A.1 Examples of variable used in the analysis of convergence

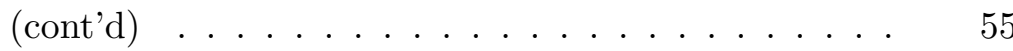

3.1 Primary studies included in the sample . . . . . . . . 74

3.1 Primary studies included in the sample (cont'd) . . . 75

3.1 Primary studies included in the sample (cont'd) . . . 76

3.2 Descriptive Statistics by primary study . . . . . . . 77 
3.2 Descriptive Statistics by primary study (cont'd) . . 78

$3.3 \quad$ Variable definitions . . . . . . . . . . . . . 82

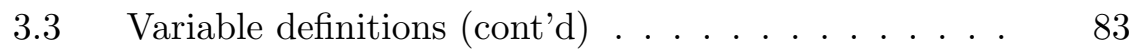

3.4 Effect sizes by direction of trade . . . . . . . . . 84

3.5 Effect sizes by methodology . . . . . . . . . . 84

3.6 Effect sizes by infrastructure category . . . . . . 84

3.7 Effect sizes by the development level of the economy in which the infrastructure is located . . . . . . . 84

3.8 Effect sizes by publication quality . . . . . . 85

3.9 Egger Tests . . . . . . . . . . . . 87

3.10 Hedges publication bias . . . . . . . . . . . 91

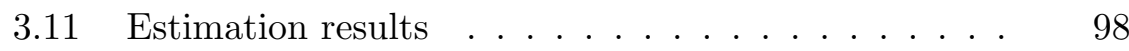

3.11 Estimation results (cont'd) . . . . . . . . . 99

3.11 Estimation results (cont'd) . . . . . . . . 100

3.12a Ranking of the studies by their mean squared errors: exporter infrastructure . . . . . . . . . . . . 101

3.12b Ranking of the studies by their mean squared errors: importer infrastructure . . . . . . . . . . . 102

3.A.1 Robustness analysis . . . . . . . . . . . 106

3.A.1 Robustness analysis (cont'd) . . . . . . . . 107 
3.A.1 Robustness analysis (cont'd) . . . . . . . . . . 108

4.1 Variable definitions . . . . . . . . . . . . . . 117

$4.1 \quad$ Variable definitions $($ cont'd) . . . . . . . . 118

4.1 Variable definitions (cont'd) . . . . . . . . 119

$4.2 \quad$ Summary statistics . . . . . . . . . . . 120

4.3 Estimation results for equation $(4.12) \ldots \ldots \ldots$

4.3 Estimation results for equation (4.12) (Cont'd) . . 133

4.4 Additional estimation results for equation (4.12) . . 134

4.4 Additional estimation results for equation (4.12) (Cont'd) 135

4.A.1 Pair RE and Heckman estimation results for equation $(4.12) \ldots \ldots \ldots \ldots \ldots \ldots \ldots \ldots$

4.A.1 Pair RE and Heckman estimation results for equation (4.12) (Cont'd) . . . . . . . . . . . . 139

4. A.2 Region codes and names . . . . . . . . . . . 142

5.1 Descriptive statistics . . . . . . . . . . 164

5.2 Estimation results for equation (10) . . . . . 167

5.2 Estimation Results for Equation (10) (Cont'd) . . . 168

5.A.1 Region codes and names . . . . . . . . . . 174

5.A.2 Measurement of political affiliation in other studies . 175 
5.A.2 Variable Measurement of political affiliation in other studies $($ cont'd $) \ldots \ldots \ldots \ldots$

5.A.3 Variable definitions . . . . . . . . . . . 176

5.A.3 Variable definitions (cont'd) . . . . . . . . . 177

5.A.3 Variable definitions (cont'd) . . . . . . . . 178

5.A.4 Correspondence of infrastructure investment and changes infrastructure stock, Istanbul . . . . . . . . . . 179

5.A.5 Correspondence of infrastructure investment and changes infrastructure stock, Izmir . . . . . . . . . . 180

5.A.6 Correspondence of infrastructure investment and changes infrastructure stock, Bursa . . . . . . . . . . . . 181

5.A.7 Correspondence of infrastructure investment and changes infrastructure stock, Ankara . . . . . . . . . . . 182

5.A.8 Correspondence of infrastructure investment and changes infrastructure stock, Kocaeli . . . . . . . . . . . . 183 


\section{List of Figures}

1.1 Night map, Turkey $(2012) \ldots \ldots \ldots \ldots$. . . . . 1

2.1 Sigma convergence represented by the coefficient of variation, and TPI (millions), 1990-2011 . . . . . . . 32

$2.2 \quad$ Moran significance map . . . . . . . . . . . . 33

2.3 GVA per capita and the growth rate of GVA per capita, $1999 \ldots \ldots \ldots \ldots \ldots \ldots$. . . . . . . . . . . 34

2.4 GVA per capita and the growth rate of GVA per capita,

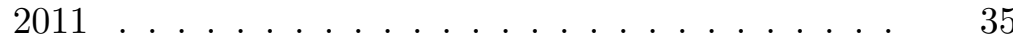

2.A.1 Moran scatterplot: Per capita GVA, 1990 (Moran's I: $0.249) \ldots \ldots \ldots \ldots \ldots \ldots \ldots$

2.A.2 Moran scatterplot: Per capita GVA, 1999 (Moran's I: $0.332) \ldots \ldots \ldots \ldots \ldots \ldots$

2.A.3 Moran scatterplot: Per capita GVA, 2011 (Moran's I: $0.353) \ldots \ldots \ldots \ldots \ldots \ldots \ldots$

3.5.1 Quantile Plots of the Infrastructure Elasticity of Trade. $\quad 79$

4.1 Exports, Turkey (constant 2005 millions of USD). . . 121 
4.2 Public investments in transportation and communication, Turkey (constant 2005 millions of USD). . . . . 121

4.3 Gross value added, Turkey (constant 2005 billions of USD). . . . . . . . . . . . . . . . . 122

4.4 Exports and public investment in transportation and communication, Turkey (constant 2005 millions USD).

4.5 The natural logarithm of regional exports for the five largest regions, Turkey (constant 2005 millions of USD).

4.6 The natural logarithm of public investments in transportation and communication for the five largest regions, Turkey (constant 2005 millions of USD). . . . . . . . 124

$4.7 \quad$ Regional exports . . . . . . . . . . . . . . 125

4.8 Public investment in transportation and communica-

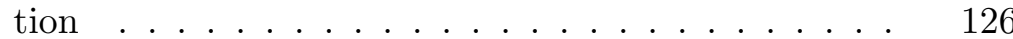

4.A.1 NUTS-2 level regional map of Turkey. . . . . . . . . 143

5.1 GVA and public investments in transportation and communication, constant 1998 national currency (billions), Turkey. . . . . . . . . . . . . . . . . 153

5.A.1 Comparison of original and modified data . . . . . 172 


\title{
CHAPTER 1
}

\author{
Introduction
}

\subsection{Background and Relevance}

This dissertation presents four research papers. While three of these studies focus on the Turkish regional economies, one of them presents a meta-analysis on primary studies which in turn use data from all over the world. Each of these papers focuses on one of the following topics: convergence, trade, and the spatial allocation of public capital. The common point of all presented studies is the concept of transportation and communication infrastructure as a key regional policy tool.

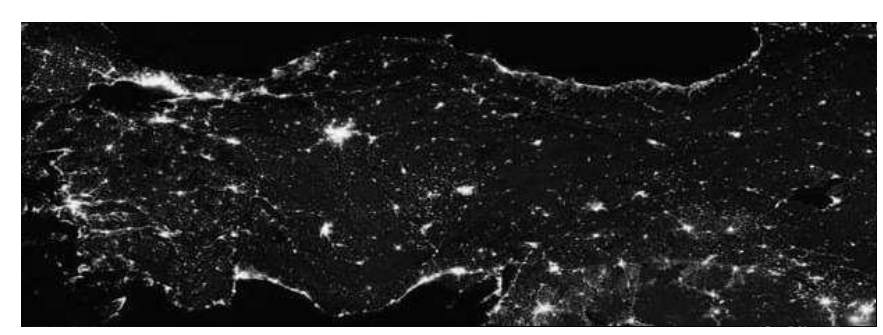

FIGURE 1.1

Night MAP, TURKey $(2012)^{1}$

${ }^{1}$ Retrieved from http://www.nasa.gov/mission_pages/NPP/news/earth-at-night. html\#.U4ozA_mSySq at 5 May, 2014. 
Pictures of the Earth's night lights taken from space are frequently used to visualize patterns relating to the economic geographies of countries and regions. Figure 1.1 shows the spatial pattern of the economic activity in Turkey by photographing the distribution of the usage of artificial light. The three largest economies in the country are noticeable in this figure: the capital in roughly the geographical center, the financial and commercial center in the northwest, and the city of Izmir in the west emit the most amount of light. Also, the coastal strips are mostly brighter than the inland areas. Occasional nodes of economic activity can be identified throughout the rest of the country. While some of these nodes fade out to darker areas, others with sharper ends to the lights patterns in their surroundings seem more isolated. This is especially the case for the activity centers in the eastern and central areas. This picture of the spatial distribution of economic activity hints to an interesting circumstance; most of the economic activity in Turkey, the world's seventeenth largest economy as of 2013 according to The World Bank (2013), takes part only in a few regions of the country.

As put by Capello and Nijkamp (2009) "economic activity arises, grows and develops in space" which in turn is "conceived as an economic resource, as an independent production factor" and a "key determinant of local production system's competitiveness" together by being "a source of increasing returns, and of positive externalities taking the form of agglomeration and localization economies" (Capello, 2009, p.33, 40, 41). Within this context, Figure 1.1 presents a meaningful thematic picture for this dissertation which looks at (1) to what extent specific policies enhance the connectivities of regional economies and contribute to positive economic outcomes in terms of trade and regional convergence, and (2) how these policies are undertaken.

The connectivities of regional economies through transportation and communication infrastructure, together with spatial disparities, regional trade, and the decision mechanisms behind the allocation of these infrastructures across regions are key components of this dissertation. Within these contexts, "connectivity" is defined in this thesis as an attribute that enhances the transportation speed of people, goods, information and ideas among economies. The strength of regional connectivity is 
measured by indicators such as the stocks, investments, and the relative locations of various types of transportation and communication infrastructures. ${ }^{2}$ More specifically, indicators such as the density of asymmetric digital subscriber lines (ADSL's), densities of land infrastructure (such as roads, highways, and railways), capacities of point infrastructures such as airports and ports, and the locations in terms of coordinates of these infrastructures relative to those of regional urban centers are used to construct the key variables used in descriptive and empirical analyses. The theoretical fundamentals which link such variables to the concepts of convergence, trade, and public capital allocation are reviewed when setting the background of each chapter.

Transportation and communication infrastructures, because of the various ways they affect a regional economy, are considered in this dissertation as key regional policy tools. Investments in these types of public capital have often been associated with various economic advantages, and while private capital is considered in some parts of the thesis, the focus is on public infrastructure. ${ }^{3}$ Regarding public infrastructure as a whole, Munnell and Cook (1990, p.11) pointed out the generalized consensus among economists that "public capital investment makes a significant contribution to national output, productivity, growth and international competitiveness," and Duffy-Deno and Eberts $(1991,340)$ showed that public capital stock is "an important input to the regional production process, which has long-run consequences for enhancing a region's productivity, and thus its competitive advantage." Along similar lines, Nijkamp (1986, p.1) states that "the relationship between infrastructure and regional development is one of the most intriguing problems in regional policy for less prosperous areas." On the other hand, while recently there has been a shift from infrastructure-related

\footnotetext{
${ }^{2}$ In Chapter 4, I make extensive use of coordinates and record the average distances of urban centers in any given region to the closest airports and ports in the country. The provincial centers closest infrastructures do not have to be in the same region. As a result, the boundaries imposed by statistical regions as units are overcome to some extent.

${ }^{3}$ Public investments have been about $20 \%$ of gross fixed investments in Turkey during the period 2008-2013. Source: Kalkinma Bakanligi (Ministry of Development), retrieved from http://www.kalkinma.gov.tr/Pages/TemelEkonomikGostergeler.aspx at 6 October, 2014.
} 
regional policy research to innovation-focused approaches, the topic of infrastructure remains highly relevant in the context of economies where regional infrastructure deprivation is still present.

Together with the economic outcomes created by the presence and enhancement of transportation and communication infrastructure, this dissertation also looks at why some infrastructure is present in certain regions more than others. Thus, the approach on the causal mechanisms comes from two directions: the impacts of infrastructure on a regional economy, and the determinants of the allocation of infrastructure across regions by central planners. For the latter approach, political factors are taken into account in the form of regional affiliation to the central government (measured using regional election result data), which could be seen as the lobbying power of a given region.

While the concepts of convergence, trade, and resource allocation are integral parts of the studies presented, infrastructure remains as the main emphasis of this dissertation. In other words, transportation and communication infrastructure is the nexus where all four research questions of the four studies stem from. In terms of case specificity, three out of the four studies - represented as separate chapters in the dissertation focus on the regional infrastructure policies and their outcomes in Turkey. Therefore, for similar upper-middle income economies, the dissertation presents policy relevant suggestions within the context of regional infrastructural policies. This can be argued especially for economies far from a near-to-satiation level of infrastructure.

Aside of the three case-specific studies, a meta-analytic research presented in the thesis aims to combine and synthesize the research findings of many previous studies on how the trade performances of countries and regions can be enhanced through policies related to infrastructure. As a result, a diverse collection of methodologies have been used in answering the research questions which are (1) to what extent internet infrastructure reduces regional disparities? (2) based on previous research, what is the statistically synthesized impact of trade-related infrastructure on trade? (3) to what extent trade-related infrastructure impacts on regional export performance in Turkey? and (4) to what extent political influences 
determine the regional allocation of transportation and communication public infrastructure? These questions were addressed using methodical approaches such as dynamic panel estimation, meta-analysis, gravity models, and spatial econometrics.

In the three studies on Turkish regions, the data used is from the 26 statistical regions of Turkey, an upper-middle income country that has a relatively large degree of regional variation in terms of economy, geography, and culture (The World Bank, 2014). For the meta-analytic study, the underlying data set is the result of a coding process using a large pool of previous statistical findings in the literature. The dissertation builds upon the theories regarding regional convergence, trade, and the equity-efficiency trade-off in relation to the regional allocation of transportation and communication public infrastructure. The objectives and findings of the presented studies are discussed in more detail within this introduction chapter.

The results presented in this dissertation stem from a collection of innovative components. For instance, focusing on internet infrastructure as a modern mode of communication infrastructure in assessing regional per capita income convergence, providing meta-analytic results on the infrastructure-trade relationship, and special focus on the implications of infrastructure enhancements in the Turkish regions within the last decade provide new approaches and case specific results to the relevant academic strand of literature. Moreover, recognizing the dependencies between capital allocation decisions (in Chapter 5), or using an interaction variable approach to see how the speed of convergence is affected by the main explanatory variable of interest (in Chapter 2) are smaller yet innovative contributions. I further discuss these novelties in the remainder of the dissertation in detail.

As previously stated, all presented studies in this dissertation meet in a specific nexus which I label "connectivity-enhancing infrastructure." This wide concept includes transportation infrastructure such as roads, highways, railroads, public and private ports, and airports. Additionally, communication infrastructure enters this concept in the form of internet groundwork. 
The research questions that meet in the nexus of connectivity-enhancing infrastructure are concerned with regional per capita income convergence, trade (imports and exports), and the allocation of public capital across regions. For instance, regional per capita income convergence in Turkey is examined from the point of view of regional infrastructural policies by augmenting the unconditional convergence equation with three infrastructure variables: internet, road/railroad, and airport infrastructure. Similarly, the determinants of the public capital allocation across Turkish regions is examined from the point of view of public investments in transportation and communication infrastructure. On the other hand, trade is examined from the point of view of not only infrastructure endowment, but also by looking at the specific locations of point infrastructures within regions. The latter approach is presented in Chapter 4 which focuses on Turkish regions, while a more general statistical synthesis of research results regarding the infrastructure-trade relationship is presented in Chapter 3.

The four individual studies are closely related to each other in terms of their findings - aside of their relation through the central concept of infrastructure. Trade performance is often seen as related to economic growth which in turn leads to the mechanism of convergence/divergence of per capita income. On the other hand, the allocation of public capital by a central government is a direct and exogenous policy instrument that can be expected to impact on a regional economy, leading to implications regarding growth, convergence, and trade. Therefore, from a regional policy-making perspective, specific types of infrastructures and their specific impacts on convergence and trade outcomes are researched. As the results presented in the dissertation have strong implications regarding these impacts, the relevance of the decision mechanisms behind regional infrastructural policies is underlined and studied using the regional economic data from Turkey in the final empirical chapter.

As a result, the dissertation can be seen as presenting three studies regarding the outcomes or infrastructure-related regional policies and one study on the inputs to the decision process of regional infrastructure allocation: Chapter 2 (Can Internet Infrastructure Help Reduce Regional Disparities? Evidence from Turkey), Chapter 3 (Infrastructure and 
Trade: A Meta-Analysis), and Chapter 4 (Infrastructure and the International Export Performance of Turkish Regions) focus on the outcomes in terms of convergence and trade, while Chapter 5 (Public Investment and Regional Politics: The Case of Turkey) focuses on the determinants of the regional allocation of transportation and communication infrastructure.

\subsection{Research Outline}

\subsubsection{Internet infrastructure and regional disparities (chapter 2)}

Per capita income disparities are commonly researched either in individual or regional/country scale. Regarding how region-centered approaches relate to individual-centered ones, Armstrong and Taylor (2000) and van Dijk et al. (2009) point to a dilemma of "place prosperity" versus "people prosperity." In this regard, van Dijk et al. (2009) claim that even though social security programs that target individual inequalities may also contribute to decreasing regional disparities (because lagging regions will have more recipients of support programs), "place prosperity" still should be independently emphasized as pursuing only "people prosperity" may have "unwanted indirect effects." Similarly, Capello et al. (2011) draw attention to how a region, may be "pushed out of business" if it has lower efficiency and competitiveness in all sectors compared to other regions, which may lead to unemployment, emigration, and even desertification.

In the light of these views, it is not surprising that the topic of regional disparities has been drawing much attention. For instance, Armstrong and Taylor (2000) argue that regional disparities hinder the attainment of national policy objectives. According to the authors, some problems caused by regional inequalities are: inefficient use of industrial and commercial land in disadvantaged regions due to lack of economic activity, excess demand for social infrastructure and public services in rapidly growing urban areas, lack of adequate job opportunities, under-utilized 
social infrastructure in areas that are losing population, and dissatisfaction and resentment due to disparities in living standards. Moreover, for the country in focus, Turkey, Filiztekin (2008) identifies more specific disadvantages created by inequalities among regions such as congestion due to migration from lagging regions to richer ones, environmental concerns, crime, and high residential rents. The author also points out how these inequalities undermine the perception of fairness regarding job opportunities - especially among the young individuals - and leads to a loss of trust within the population. As a result, disparities - not just in terms of income, but also in regard to social and demographic aspects - require sound regional policies (Capello and Nijkamp, 2009).

On the other hand, it has also been argued that the diffusion of information among economies contributes to the homogenization of regions through different mechanisms such as reducing the gap of information between markets, attracting resources from other economies, and making institutions and culture more similar across regions (Carey, 2008; Breuer et al., 2014; Ding et al., 2008). Following these arguments and combining the concepts of information diffusion and infrastructure, Chapter 2 makes the key assumption that regional internet infrastructure increases the speed of information flow between regions (i.e. reduces the cost of the transportation of information), leading to a reduction in regional disparities. The hypothesis that better internet infrastructure helps regions to converge in efficiency, represented as per-capita income is tested. The results of this chapter which presents the first study in the literature linking internet infrastructure and per capita income convergence, suggest that conditional convergence in Turkish regions holds for the period 1999-2011, and present novel evidence that public internet infrastructure increases the speed of convergence of a regional economy to its steady-state, and contributes to making region-specific steady-states more alike.

The geographical distribution of economic activity itself is strongly subject to the concentration of specialized industries in space, an occurrence which could result if there are increasing returns to concentration among other "centripetal" forces that override the disadvantages and difficulties for agents to move to the nodes of agglomeration (Krugman, 1991). 
In relation to this view, Chapter 2 also shows that the Turkish economic geography demonstrates a significant core-periphery pattern and a prominent spatial cluster of poorer regions.

\subsubsection{A meta-analysis on infrastructure and trade (chapter 3)}

Transport costs play an important role in trade and the spatial arrangement of economies (Bröcker, 2002). The most commonly used notion of transport costs in the trade literature is based on the "iceberg melting" type costs defined by Samuelson (1954). This view of trade costs assumes that part of the shipped goods from origin to destination melts away or evaporates on the way (Fujita et al., 2001). Defining transport costs as broadly as possible, these costs are often seen by researchers as not only the costs of moving people and goods around, but also as the cost of transporting information (Appold, 1995; Suda, 1997), and in a spatial context, regional infrastructure is seen as "a necessary basis for development of profitable enterprises producing goods and services for outside markets" (Hoover and Giarratani, 1971).

In Chapter 3, a new research question is posed without focusing at one specific country or region. Especially within the last decade, a fair number of studies have looked empirically at how infrastructure, and more specifically, transportation and communication public infrastructure, influences trade flows. In this chapter, meta-analytic methods including meta-regression analysis is used with the purpose of synthesizing the statistical results from previous research regarding this relationship between infrastructure and trade, as the first such attempt in the trade literature. For this study, estimates of the infrastructure elasticity of imports or exports from thirty-six studies are collected. These studies yielded 542 "effect sizes." For each effect size, the study characteristics such as the econometric methodology, sample properties, and information on included covariates are coded. Specific attention is given to the multidimensionality of the concept of infrastructure. As a result, the 
diversity in the types and measurements of infrastructure considered in the primary studies were translated into the coding process. The effect of infrastructure on trade actually embodies four categories of effect sizes. As listed in Chapter 3, these are (1) the impact of own country infrastructure on own exports, (2) the impact of own country infrastructure on own imports, (3) the impact of partner country infrastructure on own exports, and finally (4) the impact of partner country infrastructure on own imports. The meta-analysis presented in this chapter was designed in a way such that the estimation of all these four effect sizes is possible through meta-regression analysis.

After taking into account these study characteristics and potential publication bias, the results of this meta-analysis suggest that contrary to the theoretical prediction of symmetry, empirical research has generally estimated that infrastructure enhances exports more than it does imports. In other words, the impact of own country infrastructure on own exports was estimated on average to be greater than the impact of own country infrastructure on own imports. This result raises the following question: why would the same infrastructure affect outbound trade flows differently - in this case, greater - than inbound trade flows? I elaborate on several reasons which may have led to this finding. Possible reasons include the role of home economy infrastructure in lowering costs of trade for the exporting home firms to all destinations, and possible structural asymmetries regarding infrastructure orientation towards exports and imports. Further discussion of the results is presented in the concluding remarks of the chapter.

The meta-analytic results also present evidence for the existence of publication (or file drawer) bias within this strand of literature. In other words, evidence is observed that effect size estimates with low significance are less likely to be reported and/or published. 


\subsubsection{Regional exports and infrastructure in Turkey (chapter 4)}

Chapter 4 looks at the same research question on infrastructure and trade from the meta-analytic study, but focuses on the case of Turkish regions and their international exports to world countries during the period 2002-2010. This is a period of relatively higher infrastructure investment and higher export performance in Turkey. However, the link between export performance and trade-related infrastructure has not been previously studied in the regional level for this country. The reason for this could be the highly fragmented nature of regional infrastructure data on Turkish regions. Due to this high level of fragmentation regarding the data sources, for conducting the study presented in this chapter, data was collected on port, airport, and internet infrastructure from various resources on top of the relatively more available land transportation infrastructure data. For point infrastructures such as private ports, public ports, and airports, location data was recorded using the coordinates of these infrastructures.

The estimation strategy in this chapter is based on the Anderson and van Wincoop (2003) gravity model. More specifically, the estimation uses the export flows from Turkish regions to importing partner countries. The models presented take into account the generally included core explanatory variables in trade models as observed in the preceding metaanalytic chapter. The integral components of the gravity model, such as importer and exporter GDP figures, bilateral distance, and common border dummy variables all yield coefficients with the commonly observed expected signs and significances when explaining bilateral trade flows.

As part of the spatial focus of this study, the average distances to the nearest point infrastructures such as public and private ports and airports from urban centers were taken into account. In other words, attention is given on the geographical distribution of public and private infrastructure within regions and the accessibilities of regional economic agglomerations to these infrastructures. This is because the statistical Turkish regions used as the spatial units of analysis in this chapter consist of at least 
one and at most six administrative units. However, the boundaries of administrative regions may be different than the corresponding functional regions (Johansson et al., 2001). As a result, it is of high relevance to consider the distances from the urban centers of the administrative units which construct the statistical regions to the point infrastructures that are spread across the country in general. This nodal approach that is taken in Chapter 4, aims to "break free" to some extent from the boundaries imposed by the statistical regions as presented in the official data sets, and move towards the idea of a functional region which is "characterized by a high frequency of intraregional economic interaction" (Karlsson and Olsson, 2006).

The results of this chapter underline the importance of air transport capacity, land infrastructure, and private port presence in terms of density and/or relative location. Aside of the infrastructure related implications, this chapter also finds that the European Union's Customs Union agreement with Turkey has significantly and positively influenced the export performances of Turkish regions to the countries that are within this union (including those that entered the union at later stages). The results and their implications on the regional trade-related infrastructure policies regarding Turkish regions are further discussed within this chapter.

The previously introduced three chapters present evidence on how different infrastructure types lead to various economic benefits. In the following and final empirical chapter (Chapter 5), the dissertation proceeds to examine the regional infrastructural policies from the other way around by looking at how transportation and communication infrastructure is allocated among the regions in Turkey. This is done by especially taking into account the possibility of political influences. 


\subsubsection{Political influences (chapter 5)}

As stated earlier, the aim of this chapter is to assess the regional infrastructural policies form the viewpoint of a regional policy-maker. Instead of focusing on the economic outcomes of infrastructure related regional policies, this chapter aims to understand how decisions regarding the regional allocation of transportation and communication infrastructure in Turkey are made. In this regard, (Armstrong and Taylor, 2000) state that "regional policy exists because of the persistence of regional disparities in a wide range of variables, which have a profound effect on the economic welfare of a nation's regions," identifying regional policy as "an important component of a broader and more comprehensive economic policy embracing the whole economy" (Armstrong and Taylor, 2000, p.203). In this regard, to maximize country welfare, policies are implemented with the purpose of influencing the distribution of economic activity among regions, and changes in this distribution imply important consequences for the inhabitants of the country as a whole (Hoover and Giarratani, 1971).

Armstrong and Taylor (2000) classify public investments in infrastructure as an instrument to revive the disadvantaged regions based on the "interventionist approach" in contrast to the "free market approach." They clarify this view by stating "the interventionist approach argues that it is vitally important to improve the stock of social infrastructure in high-unemployment areas in order to improve their competitiveness" while the free market approach "... views the regional problem as being the result of market inefficiencies, a lack of entrepreneurial 'culture' and excessive state intervention," and argues that regional policy needs to be minimal (Armstrong and Taylor, 2000, p.210, 213). The free market approach was first emphasized in Britain during the 1960's when regional policy was seen as a method to achieve faster regional growth versus the previous approaches that focused on reducing regional disparities (Armstrong and Taylor, 2000). These contrasting approaches imply the classic equity-efficiency trade-off in regional policy-making. Which is an integral concept in Chapter 5. 
Within the context of regional policy-making, infrastructure investments have been highly discussed. Bröcker and Rietveld (2009) state that "... infrastructure investment plans are often motivated by regional policy goals. They are intended to benefit lagging regions" (Bröcker and Rietveld, 2009, p.152). Such benefits of infrastructure, more specifically, of transportation infrastructure, are argued to exist in various ways. For instance, through helping firm establishment (van Dijk et al., 2009), increasing income, and reducing unemployment (Armstrong and Taylor, 2000). Within this perspective, improving transport infrastructure is listed by Armstrong and Taylor (2000) among regional policies which were seen as a principal part of national policy to improve competitiveness and economic efficiency in Britain since the early 1990's.

As Armstrong and Taylor (2000, p.2) state, "the government's role in transferring income between regions plays a very important part in determining regional standards of living and the quality of life more generally." Together with considering the above discussion on equity and efficiency, Chapter 5 elaborates on the literature focusing on how political influences affect this trade-off. The main finding of this chapter is that infrastructure allocation in Turkey has been influenced by the political affiliations of the regions to the central government during the period 1999-2011. Given the presence of a prominent ongoing public debate in the country, this chapter provides novel academic evidence regarding the allocation decisions which the different parties in this debate (such as non-governmental organizations and opposition parties) can refer to. While the preceding studies in the dissertation using Turkish data show how improvements in infrastructure provide certain economic benefits, as the concluding chapter this final elaboration suggests that these improvements have been subject to political influences.

\subsection{Generalizability and Policy Significance}

The findings from the three case-specific studies can be used for policy advice in economies with structural characteristics similar to those of 
Turkey. In terms of economic activity, or surface area, Turkey is not among the extremes in the global distribution. Its experiences should be generalizable to countries within the same income group with relatively similar populations and areas. The constraints of such generalizability would be relaxed or tightened by the subjective interests of policy makers. More specifically, countries that aim to reduce the disparities among their regions, or those who aim to increase their trade performances given that they are structurally similar to Turkey - can consider the results presented in this dissertation.

The first empirical chapter in the dissertation, Chapter 2 builds on the theories regarding the diffusion of knowledge over space and how this can lead to the homogenization of regional economies. Countries and regions may experience negative economic outcomes due to being excluded from digital networks. While full exclusion is becoming rare in the modern digital landscape, in the developing world there are areas where varying degrees of difficulties in terms of internet access is a reality. The results of this chapter find that improved regional internet infrastructure has contributed to the regional per capita income convergence in Turkey in the period 1999-2011. These results obtained on Turkish regions could be generalizable to other countries where internet infrastructure density is uneven across space. The results could imply that for such economies, pursuing better digital linkages among regions in order to accelerate the diffusion of information on markets, institutions, culture, etc., could help reduce per capita income disparities. Of course, a prerequisite of pursuing such regional policies is that regional income disparities within an economy are seen as a problem by policy-makers.

The meta-analytic study presented in Chapter 3 has more general implications as it includes studies done for a large range of cases: the primary studies include case-specific ones as well as global level research. Therefore, it is reasonable to assume that findings not only from a specific region, but the world as a whole are represented in the data set used in the study presented in this chapter. Moreover, the employed meta-regression model takes into account a wide range of study characteristics including the level of development of the economy, allowing for the prediction of an (estimated) infrastructure elasticity of trade 
that will result from a not yet undertaken study. As a result, the study presents specific policy implications for economies that aim to reduce their current account deficits, such as giving more weight to trade-related infrastructure, together with providing further implications regarding the type and the choice of location for infrastructure investments.

The study on the effect of trade-related infrastructure on the export performances of Turkish regions, presented in Chapter 4, underlines the significance of infrastructural improvements and location choices. While infrastructure deprivation is no longer an issue for most economies in the developed world, for other parts of the world the topic still draws much attention. A prominent example is Africa where according to a report by the Commission for Africa (2005), transport costs are almost twice compared to a "typical Asian country." Infrastructure density and the linkages between infrastructure and economic centers can be seen as vital for economies who seek to increase their export performances based on previous research results as synthesized in Chapter 3. In this regard, the Turkish experience from (roughly) the last decade examined in Chapter 4 can add to the evidence leading to efficient regional policy measures that can be implemented.

A final policy related outcome is that the allocation of infrastructure in Turkey should be made free from political influences so that country welfare can be maximized through higher precision in a purely economically motivated trade-off between regional efficiency and regional equity. Through this result observed in Chapter 5, the dissertation provides valuable and novel empirical evidence to actors in Turkey such as nongovernmental organizations and political opposition parties who can advocate for the reduction of such influences. 


\section{CHAPTER 2}

\section{Can Internet Infrastructure Help Reduce Regional Disparities? Evidence from Turkey ${ }^{1}$}

\subsection{Introduction}

It is generally thought that the Solow (1956) and Swan (1956) neoclassical model of long-run growth - because of its assumption of diminishing returns - implies that economies should eventually converge to a common (and unique) steady-state growth rate of per-capita income (Islam, 2003). ${ }^{2}$ In other words, economies with relatively less income percapita should grow faster than richer ones until all economies converge to a steady-state per-capita income growth rate of zero. On the other hand, in the presence of equally shared exogenous labor-augmenting technological progress, this process would imply convergence to a positive growth rate. If, in addition, the aggregate production functions of all economies are assumed to be identical, convergence should also occur in terms of per-capita income level (Islam, 2003).

\footnotetext{
${ }^{1}$ This chapter is the result of a joint work with Denis de Crombrugghe. The title and content of this chapter is identical to the UNU-MERIT working paper with serial number 2014-078, co-authored by Denis de Crombrugghe. This study has also been presented at the UNU-MERIT conference "Future Perspectives on Innovation and Governance in Development" held on 26-28 November 2014 in Maastricht, the Netherlands, available at http://www.merit.unu.edu/wp-content/docs/25years/parallel1.php.

${ }^{2}$ While the neo-classical growth theory (NCGT) predicts the convergence of a single economy to a stable dynamic equilibrium, this notion has been related to convergence across economies in the subsequently developed convergence literature (Islam, 2003).
} 
The assumptions of equal access to common technology, and identical production functions are not unreasonable in cases where the units of analysis are sub-national regions. Barro and Sala-i-Martin (2003) point out that even though technological and structural differences may exist between regions, these differences can be expected to be less than those between countries, and therefore convergence to similar steadystates would be more likely. In this study on Turkish regions, while we do observe sufficient homogeneity that allows for regional convergence, we also see that the speed of this process is only considerable when region-specific conditions are accounted for.

The conditions that influence the process of convergence can be considered in two categories: those that influence convergence through their impact on regional economic growth, and those that play a similar role through enhancing the connections between economies. In this regard, the role of public and human capital have been highly emphasized in the growth literature among a large number of other variables (Leon-Gonzalez and Montolio, 2004). Stemming from growth models, convergence equations have been often augmented by these two types of capital. Examples of such convergence equations are found in Button (1998); Lall and Yilmaz (2001); Leon-Gonzalez and Montolio (2004); Ding et al. (2008); Del Bo et al. (2010), and Onder et al. (2010) among others, where the role of especially public capital is examined.

In this study, we focus on a specific class of public capital which is internet infrastructure, as an enhancing factor of regional telecommunications capacity. It can be seen that in many studies, communication infrastructure is often grouped within the same infrastructure category with transportation public capital. However, within the encompassing definition of public capital, the role of communication infrastructure can be considered to be different from other infrastructure types (Robins and Gillespie, 1992; Carey, 2008). The distinction between communication and transportation increasingly needs to be taken into account as these two categories are becoming more and more divergent from each other with technological progress: while transportation capabilities reduce travel time and effective distance, communication is often argued to transcend space and time, at least to some extent, by allowing informa- 
tion to travel instantly. Carey (2008) suggests that it was as early as the invention of the telegraph when communication and transportation first started to be clearly distinguished from each other, and observes a significant spatial economic consequence of this communication technology: the telegraph had lessened the gap in the market prices in different locations, reduced the "information gap" between stock markets, giving rise to "everywhere markets and everytime markets" Carey (2008, p. 169). In similar lines, Dokmeci and Berkoz (1996) observe that telecommunications has a strong impact on financial services which stimulate capital flows, which in turn influence the economic geography. In more general terms, within the context of European regions, Tranos (2012) identifies information and communication technology (ICT) infrastructure as a necessary condition for economic development.

In order to further explore this proposed relationship between telecommunications and spatial differences, we focus on the $\sigma$-convergence and $\beta$-convergence ${ }^{3}$ of Turkish regions in relation to regional internet infrastructure. While it is common to use phone subscription data as an indicator of telecommunication infrastructure, ${ }^{4}$ we focus on a more modern medium of communication measured by the density of of asymmetric digital subscriber lines (ADSL's). Previous evidence regarding the telecommunications-convergence relationship, especially from a modern technology perspective, is almost non-existent (Forman et al., 2009). In this regard, our study presents novel evidence on how improved internet capabilities of regions may help reduce regional disparities. ${ }^{5}$

We examine the absolute and conditional $\beta$-convergence (or divergence) processes for the years 1999 through 2011 by taking two other connectivity-enhancing infrastructure categories into account along with internet infrastructure: air and land transportation infrastructure. $\sigma$ convergence on the other hand, is examined for a longer time period spanning 1990-2011. ${ }^{6}$

\footnotetext{
${ }^{3}$ These concepts are summarized in Section 3.3.

${ }^{4}$ For example Ding et al. (2008) and Del Bo et al. (2010) who examine income convergence as a response to telecommunications infrastructure among other factors.

${ }^{5}$ We further elaborate on the previous research results in Section 2.2.3.

${ }^{6}$ The reason for the shorter time period used in the former case is the unavailability
} 
This study proceeds as follows: Section 3.3 elaborates on the mechanism of how modern telecommunications is expected to affect convergence, and reviews the theoretical foundations of the concepts of $\sigma$-convergence and $\beta$-convergence together with a discussion of spatial interactions. The regional patterns of the distribution of per-capita income in Turkey, and the clustering of similar regions are discussed in Section 2.3. Section 2.4 discusses the empirical strategy. The explanation of the data used is presented in Section 3.5, and Section 2.6 elaborates on the estimation results. Finally, Section 3.8 makes the concluding discussion and policy recommendations.

\subsection{Theoretical Background}

\subsubsection{The theory and analysis of convergence}

When looking at the relationship between internet and convergence, we focus on the two well established concepts in the literature: $\sigma$-convergence and $\beta$-convergence where the latter can exist as absolute or conditional convergence. In the context of regional economies, Absolute convergence is observed if the output per capita of poorer regions grows faster than that of the richer ones without being conditional on other regional structural characteristics (Barro and Sala-i-Martin, 2003). Therefore, regions are treated as structurally homogeneous. Following Barro and Sala-i Martin, ${ }^{7}$ an absolute $\beta$-convergence equation can be stated as follows:

$$
(1 / T) \ln \left(\frac{y_{i, t_{0}+T}}{y_{i, t_{0}}}\right)=\alpha-\left(\frac{1-e^{-b T}}{T}\right) \ln \left(y_{i, t_{0}}\right)+\varepsilon_{i}
$$

where $y$ is income per capita and the term on the left-hand-side is the of data for all covariates except GVA per capita for the years before 1999. For GVA per-capita, the series begins from 1990.

${ }^{7}$ Barro et al. (1991); Barro and Sala-i Martin (1992) and Sala-i Martin (1996b). 
average per-capita income growth rate of economy $i$. The subscripts $t_{0}$ and $t_{0}+T$ index the initial and final years in the data respectively, and $T$ is the number of years minus one. $\alpha$ is a constant and $\varepsilon_{i}$ is the error term. $\beta$-convergence is observed if a negative relationship, represented by the convergence factor $\beta=-\left(\frac{1-e^{-b T}}{T}\right)$, exists between the growth rate of per capita income and the initial income level (Sala-i Martin, 1996b). Therefore, a significant and negative $\beta$ estimate would imply that regions with lower initial output levels generally have higher average growth rates, and provide evidence for absolute convergence.

In addition to testing the convergence hypothesis, two additional issues are of interest: (a) the speed of convergence which is defined as the rate an economy approaches its steady-state (Barro et al., 1991) - represented by the term $b$ in equation (2.1) - and (b) the half-life of convergence which is defined as "the time span which is necessary for current disparities to be halved" Monfort (2008, p.4), and computed as $\frac{\ln (2)}{b}$ (Arestis et al., 2007). Thus, for all estimation results that we present in this study, we report the associated convergence speeds and half-lifes and discuss their implications. Expressing equation (2.1) in levels leads to

$$
\ln \left(y_{i, t_{0}+T}\right)=\theta+(1+\beta) \ln \left(y_{i, t_{0}}\right)+\nu_{i}
$$

where $1+\beta=e^{-b T}$. The constant $\theta$ is $\alpha T$ and the error term $\nu_{i}$ equals $\varepsilon_{i} / T$. Equation (2.2) is the base absolute convergence equation we estimate in the empirical analysis.

Convergence to a common steady-state with the condition that economies share similar technologies and other structural parameters is referred to as conditional convergence (Mankiw et al., 1992). This type of convergence is observed if the relationship between $y_{i, t_{0}}$ and the rate of per-capita income growth varies across regions such that convergence exists only if they share similar structural characteristics. In other words, regions have different steady-state rates of growth towards which they converge (Barro et al., 1991). However, when estimating equation (2.1), 
structural differences between regions cannot be accounted for. Islam (1995) suggests the use of panel data methods in convergence models to control for unobserved individual effects and to avoid the omitted variable bias that may exist in cross-section regressions. Following this view, the expression of equation (2.2) in discrete form for all time periods in the data, together with the addition of time-varying covariates, leads to the conditional convergence model:

$$
\ln \left(y_{i, t}\right)=\theta+(1+\beta) \ln \left(y_{i, t-1}\right)+\sum_{k=1}^{m} \gamma_{k} x_{k, i t}+\mu_{i}+\eta_{t}+\xi_{i t}
$$

where $t$ indexes the discrete time periods in the data, $\xi_{i t}$ is the error term, and convergence is conditioned on $x_{k, i t}$, a set of $m$ regional structural characteristics $k$ for region $i$ at time $t$, and $\mu_{i}$ and $\eta_{t}$ which are the region-specific and year fixed effects respectively. ${ }^{8}$ The absolute convergence counterpart of equation (2.3) omits the region-specific conditions represented in the explanatory variables and the region fixed effects:

$$
\ln \left(y_{i, t}\right)=\theta+(1+\beta) \ln \left(y_{i, t-1}\right)+\eta_{t}+\xi_{i t}
$$

\subsection{2 $\sigma$-convergence and its relation to $\beta$-convergence}

Regarding the distinction between the two concepts of convergence Sala-i Martin (1996b, p. 1328) states that

"...the two concepts examine interesting phenomena which are conceptionally different: $\sigma$-convergence studies how the

\footnotetext{
${ }^{8}$ Random effects instead of fixed effects can be of interest in cases where units of observations are randomly drawn from a larger population. In this study, we only consider fixed effects as our data covers all Turkish regions.
} 
distribution of income evolves over time and $\beta$-convergence studies the mobility of income within the same distribution."

The evolution of the distribution of income in a group of economies is represented by the trend in the cross-sectional variance of $\ln \left(y_{i t}\right)$, denoted by $\sigma$, and $\sigma$-convergence is observed if this variance is decreasing between a given time $t$ and any future period $t+T$ over time, such that $\sigma_{t}^{2}>\sigma_{t+T}^{2}$ (Sala-i Martin, 1996a). In this regard, since $\beta$-convergence asks whether poorer economies grow faster than the richer ones, its focus is on the behavior of the "relative locations within the income distribution" (Durlauf et al., 2005). On the other hand, as stated by Durlauf et al. (2005), the focus of the $\sigma$-convergence approach is on the shape of the distribution as a whole rather than the relative locations within it.

Despite these conceptual differences, the following strong links exist between the two convergence approaches. Absolute $\beta$-convergence is a necessary condition for $\sigma$-convergence to occur: it must be due to poor economies growing faster if the income differences among regions lessen through time (Sala-i Martin, 1996a). This can be seen by taking the sample variance of equation (2.4) which yields the relation $\sigma_{t+1}^{2}=$ $(1+\beta)^{2} \sigma_{t}^{2}+\sigma_{\xi}^{2}$ between $\sigma_{t}$ and $\sigma_{t+1}$ (Sala-i Martin, 1996b). ${ }^{9}$ Sigma convergence can hold only if $-1<\beta<0$ since $\beta \geq 0$ implies divergence, and $\beta<-1$ implies a negative association between $\ln \left(y_{i t}\right)$ and $\ln \left(y_{i, t+1}\right)$ which would result with a series that could oscillate between negative and positive values, making little economic sense (Young et al., 2008), a situation also known as "leapfrogging" or "overshooting" (Sala-i Martin, 1996b). Finally, if $\beta=-1$, then $\sigma_{t}^{2}$ is equal to the constant $\sigma_{\xi}^{2}$ at all time periods and $\sigma$-convergence does not occur.

It is important to note that $\beta$-convergence is not a sufficient condition for $\sigma$-convergence. Given $\beta$-convergence, the variance in the distribution of income among economies can either increase or decrease depending on

${ }^{9}$ Our expression of the sample variance of the convergence equation is slightly different from the original formulation of Sala-i Martin (1996b) in which the coefficient on $\sigma_{t}^{2}$ is $(1-\beta)^{2}$. 
an initial level of variance with respect to the variance in the steady-state (Sala-i Martin, 1996b; Islam, 2003), and $\beta$-convergence itself can lead to an increase in income dispersion if economies have different structural characteristics such that the convergence of economies to their own separate steady-states can lead to $\sigma$-divergence (Young et al., 2008). Therefore, as an increase in disparities between economies can still persist even if $\beta$-convergence exists, this type of convergence "cannot guarantee falling variance" (Islam, 2003).

\subsubsection{Communications, geography, and convergence}

The effects of the regional communication on inter-regional equity (or inequality) has been discussed in several studies. In a recent paper, Breuer et al. (2014) observe an absolute convergence of US states in the period 1929-2011 which they argue that can be explained by the explosion of the internet and the migration to cities, resulting in the homogenization of institutions and culture, and the elimination of regional distinctions. The authors refer to this phenomenon as the "globalization hypothesis." This view is partly supported by Ding et al. (2008) who show evidence that a more traditional type of communication infrastructure measured by fixed and mobile telephone lines, has positively contributed to the conditional convergence of 29 Chinese regions from 1986 through 2002. Similarly, Del Bo et al. (2010) observe a positive sign on their telecommunication infrastructure variable (mobile phone lines) in their conditional convergence equation - where spatial dependence is considered - for European regions during the period 1995-2006.

On the other hand, it can be argued that in many economies regions are satiated in terms of such traditional forms of communication infrastructure like phone lines. The only study we are aware of which examines the relationship between telecommunications and convergence by focusing on a modern telecommunication technology is the county-level research on US firms by Forman et al. (2009), ${ }^{10}$ who observe that investments by

\footnotetext{
${ }^{10}$ This working paper is published as Forman et al. (2012), focusing on regional economic growth with less emphasis on convergence.
} 
firms on advanced internet capabilities has led to regional wage divergence in the period 1995-2000. It is clear that further evidence is needed regarding the internet-regional disparities relationship.

Within the context of Krugman's New Economic Geography (NEG) model (Krugman, 1991), Tranos (2012) and Maignan et al. (2003) argue that ICT enhancements reduce the costs of communications, which in turn could change the NEG equilibrium defined by the centripetal and centrifugal forces. ${ }^{11}$ More specifically, the generally proposed mechanism of how telecommunication affects economic geography is through the increase in the speed, and the decrease in the cost of diffusion of information between markets. Goddard (1992) identifies information as a "key strategic resource on which the effective delivery of goods and services in all sectors of the world economy is dependent" and argues that "economic transformation is being underpinned by a technical transformation in the way in which information can be processed and distributed." On the other hand, Ding et al. (2008) suggest that telecommunications infrastructure, as an input to the process of production, may positively contribute to the productivity of other inputs while "liberating economic activities from geographical restraints" (Ding et al., 2008, p.846). The authors also point out that telecommunication infrastructure can lead to the attraction of resources from other regions, contributing to economic growth. In this regard, I observe in Chapter 3 that telecommunications infrastructure has a significant and positive impact on the imports of an economy, which can be seen as a result of enhanced possibilities of accessing information on goods and resources from other economies.

Even though modern modes of communication allow space to be transcended to some extent, "... space still exists and so does time" according to Castells et al. (2007, p. 178) who argue that "...wireless communication homogenizes space." Within this context, Robins and Gillespie (1992) point out that information and communication technologies are essential to the future of cities, regions, and nations. On the other hand the authors refer to the geographical dimension of these technologies by

\footnotetext{
${ }^{11}$ In the spatial context of the NEG model, centripetal forces pull economic activity together and the centrifugal forces push it apart, as put by Fujita and Krugman (2004).
} 
stating that

"We need to acknowledge the spatial bias of new ICT's, their contribution to new patterns of homogenization and differentiation, their tendency to underpin new geographical divisions and hierarchies" (Robins and Gillespie, 1992, p. 149).

In relation to this spatial bias of ICT's, Robins and Gillespie (1992) argue that the restructuring of information and communication technologies is related to significant regional inequalities between regions. Supporting this argument, Goddard (1992) provides a real world example by arguing that the growth in information industries in south-east UK in the eighties and early nineties has led to uneven regional development patterns. In this regard, Tranos and Gillespie (2009) draw attention to how information is distributed among settlements through "digital highways" and that being part of such networks creates locational advantages. Similarly, in their assessment of wireless communications, Castells et al. (2007) point out that telecommunications infrastructure is dependent on access points in space, and that the ability to transcend time and space is determined by one's location.

Another mechanism through which the availability of information from other economies may play a role in shaping the economic geography can be through affecting regional demand for product variety which is put forward as a contributing factor to technological progress in endogenous growth models. In other words, consumers who observe a greater variety of goods and services available in other regions will demand the same for their own locations, which could in turn make the region more attractive for firms that operate elsewhere. To summarize, it can be argued that telecommunications can affect the economic geography through:

1. the reduction of the information gap between markets (Carey, 2008),

2. stimulating capital flows (Dokmeci and Berkoz, 1996), 
3. creating new patterns of homogenization (Robins and Gillespie, 1992) and homogenizing of institutions and culture (Breuer et al., 2014),

4. generating productivity spillovers to other inputs of production (Ding et al., 2008),

5. attracting resources to a regional economy from other economies (Ding et al., 2008),

6. creating locational advantages as a result of being in digital networks (Tranos and Gillespie, 2009),

7. changing the NEG equilibrium through decreasing the costs of communication (Tranos, 2012), and

8. increasing the demand for product variety and attracting firms to the region.

\subsection{Regional Patterns of Income Per Capita in Turkey}

Our interest lies more in the mobility of regions in terms of their percapita income compared to the general trend in the disparities among them. Therefore, we put greater emphasis on the $\beta$-convergence process, its speed, and half-life. On the other hand, as a useful first look, the $\sigma$-convergence process in Turkey relative to the country-wide regional connectivity-enhancing public capacity, represented by the transportation and communication public investments (TPI), is visualized in Figure 2.1. We use the coefficient of variation (CV) in GVA per capita, as it is a common indicator for examining $\sigma$-convergence. Certain sharp kinks in the two curves in Figure 2.1 are prominent. We can see the response of the investments to the 2001 crisis in the form of a sharp drop, ${ }^{12}$ followed by an increase with a change of government from coalition to a

\footnotetext{
${ }^{12}$ This was arguably the heaviest financial crisis in Turkish history.
} 
single-party structure. Moreover, we see a clear correspondence of rising TPI to falling regional disparities in more recent years. ${ }^{13}$ Therefore, a clear process of $\sigma$-convergence is apparent for Turkey for the period in question, possibly as a response to public investments targeted to enhance regional connections.

The spatial implications of this convergence process is of interest. As a preliminary exploration, we look at the Global Moran's I statistic (Moran, 1950), and its local decomposition, the Local Moran's I (Anselin, 1995), which are commonly used indicators for examining how regional interdependencies exist over space. ${ }^{14,15}$ The Global Moran's I represents the degree of linear association between a variable and the weighted average of the same variable observed in the regions in proximity (also referred to as its spatial lag) (Anselin, 1996) and can be seen as "... the simplest and most commonly used test statistics in the spatial econometric literature" (Arbia, 2006). Significant and positive global Moran's I results would imply that regions that are closer to each other have more similar per capita GVA levels than to those that are further away (Elhorst, 2012).

For the per-capita income of Turkish regions, the Global Moran's I statistics for the years 1990, 1999, and 2011 (for the per capita GVA values) are respectively $0.202,0.283$, and 0.346 and highly significant (all results have p-values less than 0.001). ${ }^{16}$ Therefore, significant positive

\footnotetext{
${ }^{13}$ On the other hand, Chapter 5 shows that in Turkey, the regional allocation of public investments in transportation and communication have been subject to political bias (created due to a preference towards regions that are politically affiliated to the government) during the period 1999-2011.

${ }^{14}$ The Global Moran's I statistic is calculated as $I=$ $\left(\frac{n}{\sum_{i} \sum_{j} w_{i j}}\right) \sum_{i} \sum_{j} w_{i j} z_{i} z_{j} / \sum_{i} z_{i}^{2}$ while local Moran's I statistic is $I_{i}=z_{i} \sum_{j} w_{i j} z_{j}$ where $z_{i}$ are deviations from the mean GVA per capita, and $w_{i j}$ measures the regional connectivity between $i$ and $j$, usually in the form of contiguity or distance of the regions $i$ and $j$ (the matrix could be either row-normalized or not) (Anselin, 1995). The above given calculation of the Global Moran statistic is for a non-standardized spatial weight matrix $W$ of which $w_{i j}$ is its element.

${ }^{15}$ See also Varga (1998) for a comprehensive review of global and local Moran's I.

${ }^{16} 1990$ is the first year in our sample where we can calculate regional GVA per capita. 1999 is the first year where we have observations for all explanatory variables and therefore is the starting year for our estimations. 2011 is the final year in our data
} 
global spatial dependence is suggested. In other words, regions with similar GVA per capita tend to be clustered around each other. In order to identify how individual regions behave in this context, the Moran scatterplots (Anselin, 1996; LeSage and Pace, 2009) are presented in Figures 2.A.1 to 2.A.3. ${ }^{17}$ These scatterplots suggest that richer regions are close to richer ones, and poorer regions to poorer ones. A nonsurprising result considering the long history of regional inequalities in Turkey. ${ }^{18,19}$

For the purpose of identifying the local spatial clusters or "hot spots" as labeled by Anselin (1995), we calculate the Local Moran's I results for all 26 regions. Local Moran's I shows the extent of significant local spatial clustering around individual regions (Anselin, 1995). Using the Local Moran's I statistics for each region, and the distribution of the regions among the four quadrants of the associated Moran scatterplot, a "Moran significance map" can be drawn (Anselin et al., 2006; LeSage and Pace, 2009).

The Moran significance maps for Turkey are presented for the years 1990, 1999, and 2011 in Figures 2.2a to 2.2c. In building the Moran significance map, we define categories of regions based on the quadrants of the Moran scatterplot as in Le Gallo and Ertur (2003) such that regions labelled "High-High" are those that have higher than average GVA per capita and are surrounded by regions with also higher values. "Low-Low" stands for regions with lower than average income per capita surrounded by similarly poor regions. Regions with lower than average per-capita income surrounded by rich regions are labelled "Low-High" while "High-Low" stands for regions that have the opposite kind of spatial association with their surrounding regions. We also include a

set.

${ }^{17}$ The calculations of the Moran's I values and scatterplots were done using the SPATLSA and SPATGSA commands in Stata developed by Pisati (2012).

${ }^{18}$ See Gezici and Hewings (2004) - who do not find evidence for convergence in Turkey for the period 1990-1997 - for a comprehensive review of convergence studies on Turkey.

${ }^{19}$ In the rest of this study, we use the term "rich" for regions with higher than average per-capita income, and the term "poor" for those that have a lower per-capita income than the country average. 
fifth category labeled "not significant" for regions with Moran's I p-values of greater than 0.10, such that only "significant local clusters" are colored (Le Gallo and Ertur, 2003).

Based on these figures, we observe (for all three years) significant patterns of spatial clustering around the largest regional economy of the country, Istanbul (TR10) in the north-west, which may be considered to include the capital Ankara in its extremity. Istanbul is a natural trade hub, connecting the maritime trade routes of the Black Sea and the Mediterranean Sea, the land trade routes from the rest of the country to the EU, and is also the commercial and financial center of Turkey. This clustering around Istanbul could also be interpreted from a coreperiphery point of view (Krugman, 1991); a core economy exists in the North-West, with peripheral economies located around it.

The Moran significance maps also identify a High-High type of clustering in the south-west of the country. This is most probably due to the area being the tourism core of Turkey. Therefore, another spatially relevant observation could be made regarding the clustering of specific industries.

An alarming and crucial suggestion of the Moran significance maps is the spatial clustering of poor regions in the East, underlining a strong spatial distinction from the rest of the country. This area, which is about one-third of Turkey, falls into the Low-Low category. To exacerbate the situation, regions that can be considered as core economies do not exist in the vicinity which can help reversing the trend of poor-poor spatial clustering, including beyond the international borders to the east and the south of this area. ${ }^{20}$ The opposite is true for the regions in the West, which share either land or maritime borders with the EU.

There are only two regions that fall into the remaining categories of High-Low and Low-High: the region of Ankara, named after the capital of the country which it contains, is a rich economy surrounded generally

\footnotetext{
${ }^{20}$ Areas under varying degrees of conflict exist in the proximity of Turkey's borders to the east such as Northern Syria, Northern Iraq, Nagorno-Karabakh, and Chechnya Moreover, the eastern region of Turkey itself was under conflict during the recent decades.
} 
by poorer ones (High-Low). This type of economy is referred to as a "diamond in the rough" by Le Gallo and Ertur (2003). Ankara used to be a small village when it replaced Istanbul as the capital in 1923, suggesting a significant exogenous intervention to its regional economy that might have caused this outcome. ${ }^{21}$ On the other hand, by 2011, Ankara had moved into the category of High-High regional economies, leaving the "diamond" category. The other regional economy that is in a similar situation in terms of its dissimilarity to its surroundings, is TR71, a neighbor of Ankara. ${ }^{22}$ This region falls into the category Low-High, a poor region close to richer regions, a type of regional economy which is referred by Le Gallo and Ertur (2003) as a "doughnut." On the other hand, the rest of the regions that are colored in white are those that do not yield significant local Moran's I statistics on the $10 \%$ level, and therefore are not subject to a significant spatial clustering. ${ }^{23,24}$

Since $\sigma$-convergence, and significant spatial clusters are apparent for Turkey, two questions that relate to these two findings can be asked: (1) how fast are poor regions catching up to the rich regions? and (2) what does the spatial distribution of income per capita look like? Figures 2.3 and 2.4 compare the per-capita income levels of the regions to the growth rates of their per-capita incomes for the years 1999 and 2011 respectively where darker colors represent higher per-capita growth rates and income levels. Clear correspondences of high incomes to low rates of growth can be seen for the earlier defined northwestern and southwestern clusters and the region TR31 in the West for the year 1999. Poorer regions who had high growth rates in this year are mainly grouped in the Northeast, North, and center-East. We can also observe that the region TR71, consistent with the Moran Significance Maps, did not grow fast despite being a low income region. In the maps for 2011, it can be seen that certain poorer southeastern regions experienced higher growth. Region

${ }^{21}$ This change in capital city status is due to the replacement of the Ottoman state by the Republic of Turkey.

${ }^{22}$ Region TR71 consists of the following provinces: Kirikkale, Aksaray, Nigde, Nevsehir, and Kirsehir.

${ }^{23}$ All maps are drawn using the Stata command SPMAP developed by Pisati (2007).

${ }^{24}$ The Stata command MERGEPOLY by Picard and Stepner (2012) for aggregating smaller scale spatial units to larger units were used to construct all maps as the original source map was in NUTS-3 level. 
TR71 which fell into the "doughnut" definition based on the Moran significance map also had high growth in this year. It is possible that if this region has continued to experience high growth, it may have moved out from the Low-High category in the period after 2011. We also see that the rich regions in the southwestern cluster together with some regions from the northwestern cluster have behaved according to the high income - low growth hypothesis.

To conclude this section, we suggest the existence of several stylized facts for Turkey for the period in focus: (1) An increasing trend in public investments in transportation and communication corresponds to a decreasing trend in regional per-capita income disparities, and (2) despite the decreasing trend in regional disparities, the spatial clustering of poor and rich regions is persistent. On the other hand, it should be noted that these maps are merely snapshots in time and are solely for giving preliminary descriptive information. Nevertheless, these two stylized facts set the stage for absolute and conditional $\beta$-convergence analyses which we present in the next section.

Figure 2.1

Sigma CONVERGENCE REPRESENTED BY THE COEFFICIENT OF VARIATION, AND TPI (MILLIONS), 1990-2011

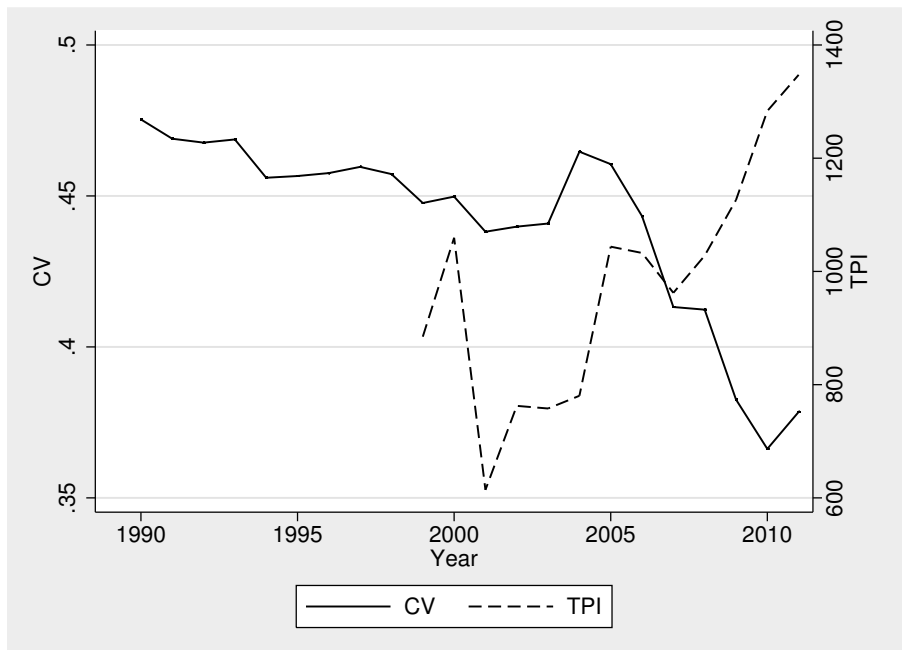


Figure 2.2. Moran SIGNIFICANCE MAP

(A) 1990

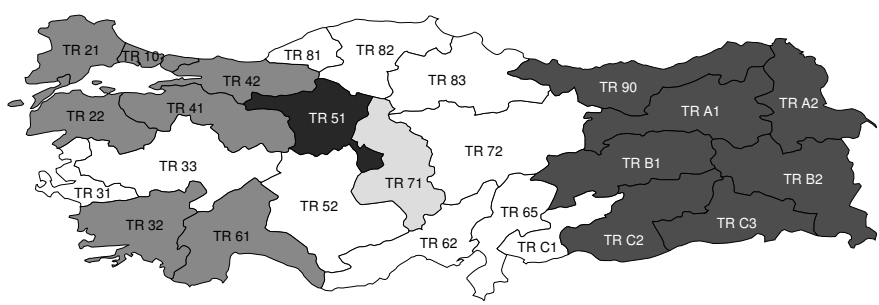

$\square$ Low-High

$\square$ High-High

-High-Low

$\square$ Not significan

(в) 1999

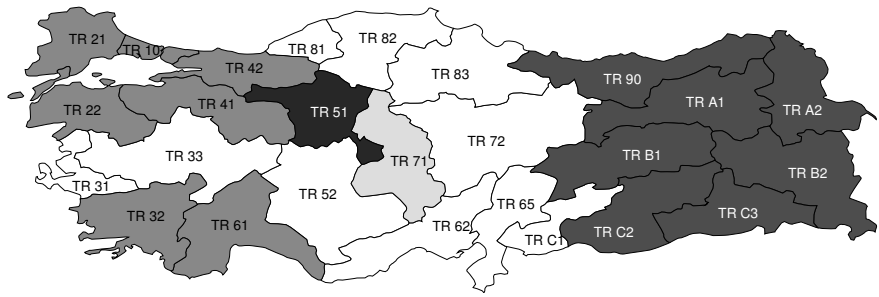

$\square$ Low-High

$\square$ High-High

Low-Low

Not significan
$\square$ High-Low

(C) 2011

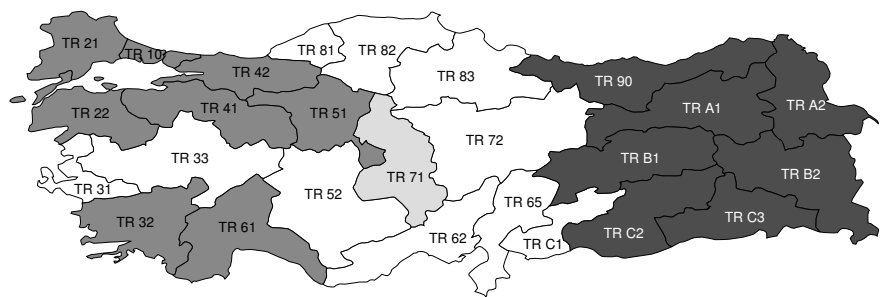

$\square$ Low-High

$\square$ Low-Low

High-Low (No region in this category)

$\square$ Not significan 
Figure 2.3. GVA Per CAPItA AND the Growth RAte of GVA PeR CAPITA, 1999

(A) GVA PER CAPITA

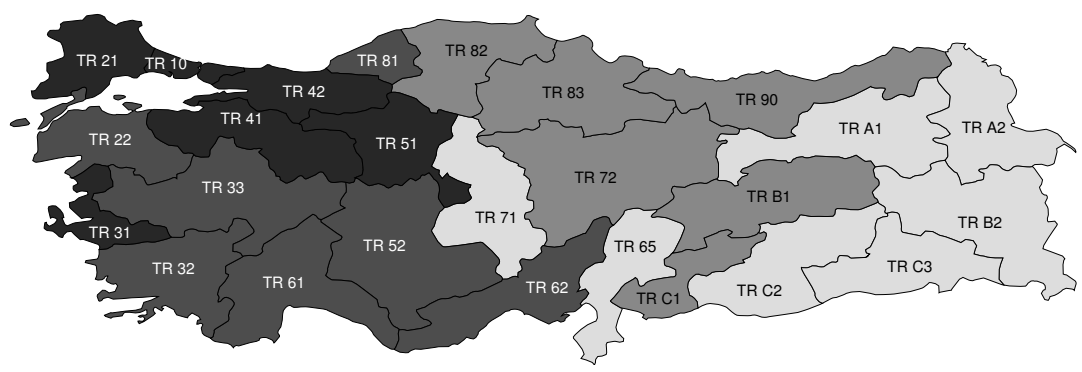

$\square[364.5,535.8]$

- $647.0,946.3]$

- $(946.3,1637.1)$

(B) Growth Rate

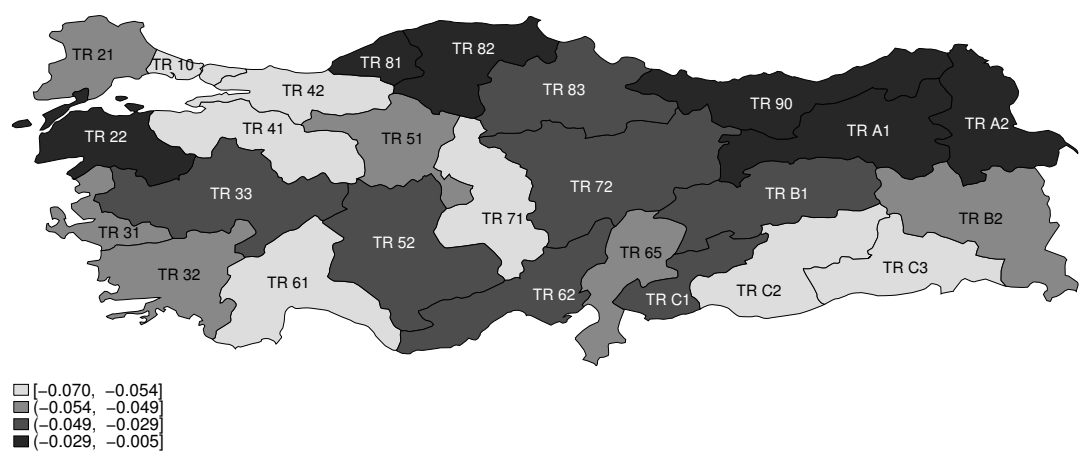


Figure 2.4. GVA PER CAPITA AND THE GROWTH RATE OF GVA PER CAPITA, 2011

(A) GVA PER CAPITA

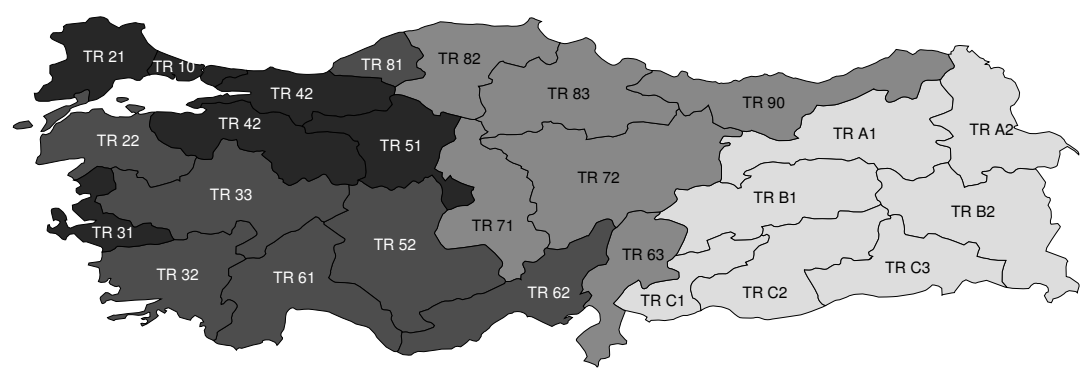

$\square[520.6,870.6]$
$\square(870.6,1048.7]$

$\square(870.6,1048.7]$

ㅁ(1048.7, 1495.9]

(B) Growth rate

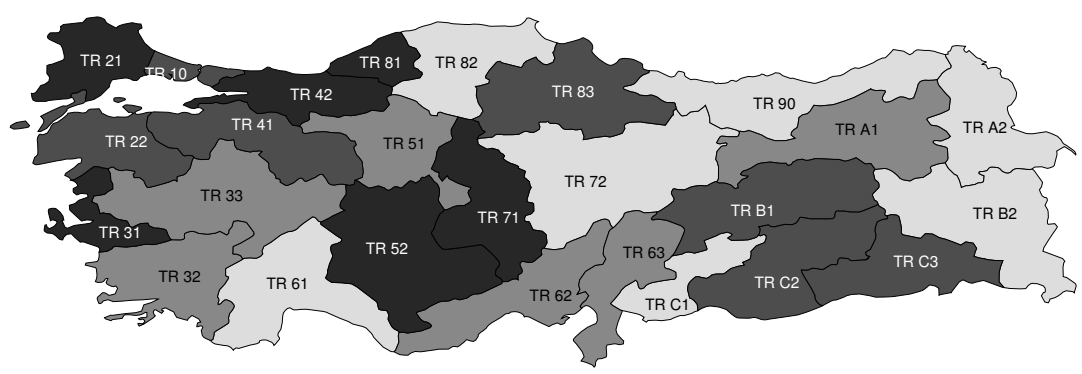

$\square[-0.026,0.035]$

$\square(0.035,0.050)$

G $(0.073,0.107]$ 


\subsection{Empirical Approach}

As discussed in section 2.2.3, even though communication reduces the effect of the spatial barriers to the diffusion of information, space and location remain relevant. Spatial effects ${ }^{25}$ are commonly taken into account in convergence research as can be seen in Table 2.A.1 which lists a sample of 18 convergence studies and the explanatory factors they consider. The table shows that there is a large diversity in the literature regarding the explanatory variables considered in convergence equations. Among these factors, spatial effects are commonly formalized as "spatial dependence" (Anselin, 1988) which is defined as representing "the continuity of economic phenomena in space" (Arbia, 2006, p. 16). In this perspective, Ertur and Koch (2007) argue that technological interdependence between economies exist and that the mechanism of these interdependencies is through spatial externalities and find evidence that these spatial externalities are important determinants of conditional convergence among countries. This type of dependence between economies is also highlighted by Armstrong and Taylor (2000) who point out that small economies can benefit from technical progress that takes place outside their borders due to diffusion across space. ${ }^{26}$

In the light of these views, and the suggestion of possible spatial dependence by our descriptive analyses, we begin by testing the absolute convergence hypothesis with estimating the cross-sectional equation (2.2) which does not take into account region specific characteristics. In order to see if our results are robust to the inclusion of the earlier discussed spatial effects, we augment equation (2.2) to account for spatial dependence. The spatial autoregressive model (SAR) (Anselin, 1988) adaptation of equation (2.2) takes the form

\footnotetext{
${ }^{25}$ Florax and Van der Vlist (2003) define spatial effects as a "catchall term referring to both spatial dependence and spatial heterogeneity".

${ }^{26}$ See Ertur and Koch (2007); Pfaffermayr (2012) for discussions on the integration of spatial effects to the underlying theoretical growth model.
} 


$$
\ln \left(y_{i, t_{0}+T}\right)=\alpha+\rho \sum_{j=1}^{N} w_{i j} \ln \left(y_{j, t_{0}+T}\right)+(1+\beta) \ln \left(y_{i, t_{0}}\right)+\nu_{i}
$$

where $w_{i j}$ is the element of the weight matrix $W$ of inverse distances between regions with zeros in the diagonal $\left(w_{i i}=0\right)$, and $N$ is the number of spatial units. Therefore, the SAR model hypothesizes that the per-capita income of region $i$ is partly determined by the weighted average of the per-capita incomes of the other regions, where the weight of a region is defined by its proximity to $i$. The additional parameter $\rho$ measures the magnitude of this spatial dependence. All other terms are the same as in equation (2.1).

An alternative augmentation to equation (2.2) is the spatial error model (SEM) (LeSage and Pace, 2009) where spatial dependence is hypothesized to exist through the disturbances $\nu_{i}$ :

$$
\begin{aligned}
& \ln \left(y_{i, t_{0}+T}\right)=\alpha+(1+\beta) \ln \left(y_{i, t_{0}}\right)+\nu_{i} \\
& \text { and } \nu_{i}=\lambda \sum_{j=1}^{N} w_{i j} \nu_{j}+\zeta_{i}
\end{aligned}
$$

where $\zeta_{i} \sim N\left(0, \sigma_{\zeta}^{2}\right)$ and the parameter $\lambda$ captures the spatial error dependence. A significant $\lambda$ would mean that there are spatially clustered relevant variables that are omitted in the model, resulting in error terms are not independent from each other (Ward and Gleditsch, 2008). A third form is the General Spatial Model (GSM) ${ }^{27}$ and is a combination of the above SAR and SEM specifications Kelejian and Prucha (1998):

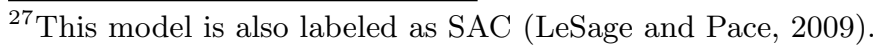




$$
\begin{aligned}
& \ln \left(y_{i, t_{0}+T}\right)=\alpha+\rho \sum_{j=1}^{N} w_{i j} \ln \left(y_{j, t_{0}+T}\right)+(1+\beta) \ln \left(y_{i, t_{0}}\right)+\nu_{i} \\
& \text { where } \nu_{i}=\lambda \sum_{j=1}^{N} c_{i j} \nu_{j}+\zeta_{i}
\end{aligned}
$$

where we assume $w_{i j}=c_{i j}$ (i.e. the same spatial weight matrix $W$ defines the connectivity between regions both in terms of their percapita incomes and their disturbances). The conditional convergence counterparts of the absolute convergence equations (2.5), (2.6), and (2.7), condition the convergence process on three regional connectivityenhancing attributes; internet infrastructure together with air, and land transport infrastructure. Our variable of interest, internet infrastructure, is measured as the number of ADSL lines in regional post offices percapita and is denoted by $c_{i}$. As discussed in Section 5.1 , the connectivity between regions is not established only through communication, but also through transportation. Thus, we include two other variables that are expected to enhance inter-regional connectivity: Air transport capacity per capita $\left(a_{i}\right)$, and an index of land transportation infrastructure density $\left(r_{i}\right)$. This set of explanatory variables correspond to the term $\sum_{k=1}^{m} \gamma_{k} x_{k, i t}$ in the panel conditional convergence equation (2.3) such that $\sum_{k=1}^{m} \gamma_{k} x_{k, i t}=\gamma_{1} \ln c_{i t}+\gamma_{2} \ln a_{i t}+\gamma_{3} r_{i t} .{ }^{28}$

In order to explore how the rate in which a region approaches its steady-state depends on its internet infrastructure, we introduce an interaction term $\ln y_{i, t-1} \times \ln c_{i t}$ so that $\sum_{k=1}^{m} \gamma_{k} x_{k, i t}$ is now defined as $\sum_{k=1}^{m} \gamma_{k} x_{k, i t}=\gamma_{1} \ln c_{i t}+\gamma_{2} \ln y_{i, t-1} \times \ln c_{i t}+\gamma_{3} \ln a_{i t}+\gamma_{4} r_{i t}$. This implies that $e^{-b T}$, which is the marginal effect of $\ln y_{i, t-1}$ is now defined as $e^{-b\left(\ln c_{i t}\right) T}$ (i.e. the speed of convergence is a function of $\ln c_{i t}$ ) and equals $(1+\beta)+\gamma_{2} \ln c_{i t}$ where $(1+\beta)$ is the estimated coefficient on $\ln y_{i, t-1}$.

${ }^{28}$ The land infrastructure index $r_{i t}$ does not appear in natural logarithms because, as will be detailed in Section 3.5, the components of this index are in natural logarithms. 
The specifications corresponding to equations (2.5), (2.6), and (2.7) which include individual region and year effects, and the set of explanatory variables $x_{k}$ are respectively:

$$
\begin{aligned}
& \ln \left(y_{i t}\right)=\alpha+\rho \sum_{j=1}^{N} w_{i j} \ln \left(y_{j t}\right)+(1+\beta) \ln \left(y_{i, t-1}\right)+\sum_{k=1}^{m} \gamma_{k} x_{k, i t}+ \\
& \mu_{i}+\eta_{t}+\xi_{i t} \\
& \text { (SAR) } \\
& \ln \left(y_{i t}\right)=\alpha+(1+\beta) \ln \left(y_{i, t-1}\right)+\sum_{k=1}^{m} \gamma_{k} x_{k, i t}+\mu_{i}+\eta_{t}+\xi_{i t} \\
& \text { where } \xi_{i t}=\lambda \sum_{j=1}^{N} w_{i j} \xi_{j t}+\vartheta_{i t} \\
& \ln \left(y_{i t}\right)=\alpha+\rho \sum_{j=1}^{N} w_{i j} \ln \left(y_{j t}\right)+(1+\beta) \ln \left(y_{i, t-1}\right)+\sum_{k=1}^{m} \gamma_{k} x_{k, i t}+ \\
& \mu_{i}+\eta_{t}+\xi_{i t} \\
& \text { where } \xi_{i t}=\lambda \sum_{j=1}^{N} w_{i j} \xi_{j t}+\vartheta_{i t}
\end{aligned}
$$

where $\vartheta_{i t} \sim N\left(0, \sigma_{\vartheta}^{2}\right)$. The estimation results for each model are presented in Section 2.6 where the absolute convergence counterparts of all panel equations are also presented.

The growth-convergence panel equations (2.3), (2.4), (2.8), (2.9), and (2.10) are subject to the Nickell (1981) bias induced by the de-meaning of the data for each unit of observation for fixed effects estimation. This bias is of order $1 / T$ and therefore decreasing with larger time periods. 
Since our data is over a period of 13 years, we can expect a moderate degree of Nickell bias in our estimations. As will be presented in our results, since our estimates of $(1+\beta)$ are positive in all models, this bias would be negative (Nickell, 1981).

Common approaches as a remedy for this bias are the Arellano and Bond (1991a) estimator and the Arellano and Bond (1991b)/Blundell and Bond (1998) Generalized Method of Moments (GMM) estimators. However, in the context of dynamic panel models with spatial effects, Elhorst et al. (2010) conduct a Monte Carlo simulation which implies that while an Arellano and Bond (1991a) GMM approach would reduce the bias in the estimate of $(1+\beta)$, it would yield an estimate of the spatial autoregressive parameter $\rho$ with a larger bias compared to Maximum Likelihood Estimation (MLE). ${ }^{29}$ Given that the time span in our study corresponds to 13 years, and the above implication regarding the tradeoff between MLE and GMM approaches, a MLE approach is taken in estimating the SAR, SEM, and GSM based specifications under the assumption that the data is distributed normally. ${ }^{30}$ While being a commonly employed method for dealing with bias and inconsistency issues in spatial models (Elhorst, 2003), MLE is also among the common approaches used to estimate growth-convergence equations (Islam, 2003). MLE provides advantages in terms of asymptotic efficiency but on the other hand may not be robust to violations of assumptions regarding the distribution of the data. Recent studies using a MLE approach for estimating spatial panel models include Pfaffermayr (2012) who examines European regional convergence from 1980 through 2005, Baltagi and Bresson (2011) who use a panel of eighty districts of Paris from 1990-2003, Ertur and Musolesi (2012) who use a panel of 21 OECD economies and Israel over the years 1971-90, Elhorst and Freret (2009) who estimate a spatial Durbin panel model using data from ninety-three

\footnotetext{
${ }^{29}$ Elhorst et al. (2010) suggest the use of a combination of GMM and MLE based on their simulation results.

${ }^{30}$ All models with spatial terms are estimated using the Stata command SPAUTOREG written by Shehata (2011) for the cross-sectional models, and the command XSMLE developed by Belotti et al. (2013) for the panel models. The generation of the weight matrix from coordinates is done using the Stata command SPMAT developed by Drukker et al. (2011).
} 
local government departments in France over the period 1992-2000, and Lee and Yu (2010a), Lee and Yu (2010b), and Debarsy and Ertur (2010) who conduct Monte-Carlo analyses.

\subsection{Data}

The variables used and their sources are defined as follows. We use the regional GVA series in constant 1998 national currency compiled as detailed in Chapter 5. This GVA series is based on the Turkish Statistics office (TURKSTAT) data ${ }^{31}$ and is corrected for changes in spatial scale and output collection methods of TURKSTAT that took place in the 2000's. Additionally, two missing years in the data were imputed as the original data was available in NUTS-3 level GDP for the years 1987-2001 but in NUTS-2 level GVA for 2004-2011. ${ }^{32}$ The regional population data is from OECDstat. ${ }^{33}$ The variable $c_{i t}$ is measured as the number of ADSL lines in regional post offices per-capita ${ }^{34}$ and is collected from the publications of the General Directorate of PTT. ${ }^{35}$ The missing values for the number of ADSL lines for the years 1999-2002 and 2005 are predicted using the regional public investment figures in transportation and communication made in these years. Air transport capacity data is obtained from the interactive web-tool of the Republic of Turkey - Ministry of Transport, Maritime Affairs and Communication, ${ }^{36}$ and measured as the total passenger capacity in airports - as reported after the establishment date of the specific airport(s) within a region divided by regional population. The three components that are used to construct the land infrastructure density index are from TURKSTAT. The index is constructed using the first principal components of the

\footnotetext{
${ }^{31}$ www.turkstat.gov.tr.

${ }^{32}$ See appendix A1 of Chapter 5 for the details of these adjustments.

${ }^{33}$ http://stats.oecd.org/.

${ }^{34}$ For our estimations we have scaled this density by dividing population by $1,000,000,000$.

${ }^{35}$ Retrieved from http://www.ptt.gov.tr/?wapp=statistics_tr on 10 April, 2014.

${ }^{36}$ Retrieved from http://web.shgm.gov.tr/tr/havaalanlari/381-havaalanlari on 15 April, 2014.
} 
natural logarithms of road length, highway length, and railway length in kilometers per $1000 \mathrm{~km}^{2}$. The distance weight matrix used in the spatial analyses is constructed as follows. The coordinates in decimal degrees of the city in a region with the highest population is taken as the "regional center." This is done so that a more sensitive measure of regional connectivity, compared to using polygon centroids, can be achieved. The euclidean distances between regions are generated from these coordinates. The descriptive statistics of all variables used in the construction of model covariates are reported in Table 2.1.

TABLE 2.1

DEsCRIPTIVE STATISTICS

\begin{tabular}{lcccccc}
\hline \hline \multicolumn{1}{c}{ Variable } & Mean & Std. Dev. & Min. & Q1 & Q3 & Max. \\
\hline GVA (Billion TL) & 3.03 & 4.08 & 0.44 & 1.12 & 3.27 & 27.44 \\
ADSL & 113.56 & 100.88 & 2 & 44 & 160 & 665 \\
Population & $2,707,646.58$ & $1,981,422.14$ & 732,790 & $1,617,820$ & $3,048,651$ & $13,255,685$ \\
Air capacity & 1 & 1.12 & 0 & 0.18 & 1.44 & 5.18 \\
Land infrastructure index & 3.93 & 1.12 & 1.86 & 3.18 & 4.83 & 6.95 \\
& & & & & & \\
Observations: 26 regions, 13 years. & & & & & \\
\hline
\end{tabular}

\subsection{Estimation Results}

\subsubsection{Cross-sectional estimation}

Table 2.2 presents the estimation results for the absolute convergence equations (2.2), (2.5), (2.6), and (2.7). All models suggest the existence of absolute convergence for Turkish regions over the period 1999-2011, including those that take spatial effects into account. However, the estimated speeds of convergence range from about $0.8 \%$ to about $2.5 \%$, which imply a very long half-life of at least about thirty years. 
Estimates of $\rho$ in the SAR and GSM models suggest the existence of positive spatial externalities; the current per-capita income level of regions, $\ln \left(y_{i, t_{0}}\right)$ are positively impacted by the levels of $\ln \left(y_{i, t_{0}}\right)$ of the regions in their surroundings, implying that spatial proximity plays role in the growth and convergence of regions. The fastest rates of convergence are estimated by these two models (SAR: $2.46 \%$ with a halflife of about 28 years, and GSM: $2.19 \%$ with a half life of about 32 years). The GSM estimation does not find spatial correlation between model residuals but similar to SAR results, finds positive spatial dependence in $\ln \left(y_{i, t_{0}}\right)$.

On the other hand, the estimate of $\lambda$ in the SEM suggests that the errors of the model are negatively correlated in space, which could bias the estimates on the exogenous variables if this correlation is not taken into account. Additionally, the SEM estimates a lower (in absolute value) convergence factor $\beta$, a lower convergence rate of about $0.82 \%$, and a much longer half-life of about 85 years compared to the results of the Base, SAR, and GSM results. The significant estimate of $\lambda$ in SEM becomes insignificant when the spatial autoregressive term $\rho$ is taken into account in the GSM estimation. In other words, spatially correlated error terms are only observed when the spatial lag of $\ln \left(y_{i, t_{0}}\right)$ is omitted. Finally, the base model, where spatial effects are not taken into account, estimate the convergence speed and half-life to be higher than those reported by the SAR and GSM, but lower than those in the SEM results.

In order to identify the specification with higher adequacy, we report Akaike's Information Criterion (AIC), and the Bayesian Information Criterion (BIC) at the bottom of Table 2.2 aside of the log-likelihood values. While SAR and GSM models yield the highest log-likelihood values, the AIC and BIC results suggest that the SAR model fits our data better compared to the GSM specification. ${ }^{37}$ We also report separately the likelihood ratio (LR) and the Wald test p-values where the GSM is the unrestricted model in part (a) of Table 2.6. In accordance with the above results, the p-values of both tests suggest that while the GSM can

\footnotetext{
${ }^{37}$ The base model is a restricted specification of SAR, SEM and, GSM such that if $\rho=0$ SAR reduces to the base model, if $\lambda=0$ SEM reduces to the base model, and if $\rho=\lambda=0$ GSM reduces to the base model (Elhorst, 2010).
} 
be reduced to the SAR model (i.e. $\lambda=0$ ), neither the base model or the SEM model are adequate, as $\rho \neq 0$.

Therefore, the SAR model is preferred regarding the cross-sectional results. The results of the SAR model suggest positive spatial dependence in $\ln y_{i, t_{0}+T}$, implying that regional per capita incomes are positively affected by the per-capita incomes of the other regions in proximity. This result is in line with our earlier observations of spatial clustering of similar economies in Turkey and affirms the earlier hinted existence of spatial effects.

\subsubsection{Panel estimation}

We extend the cross-sectional approach to a panel form and present the results of the absolute convergence models in Table 2.3. Regional fixed effects are included in all estimations except in the first column. When fixed effects are not included, the estimated convergence speed is about $1.3 \%$, a result similar to those of the cross-sectional base model. This result, while in principle suggesting convergence, implies that this convergence is very slow with a half-life of around 55 years which can be seen as the non-existence of convergence. This slow convergence speed is observed even though the estimated coefficient on $\ln y_{i, t-1}$ is 0.987 which is only about 0.17 percentage points higher than the corresponding elasticity estimated by the fixed effects base model in column 2 .

A possible explanation to this result can be found in Arestis et al. who show that the calculation of the speed of convergence is "extremely sensitive to small changes in the estimated regression coefficients and hence may be greatly influenced by a relatively small bias on these estimates" Arestis et al. (2007, p.214). It is likely that the omission of region-specific fixed effects resulted in the estimation of such a slow speed of convergence in column 1 which is more similar to its cross-section counterpart in the first column of Table 2.2 rather than the specification with fixed effects. Moreover, as ordinary least squares estimation (OLS) may lead to biased and inconsistent results if spatial effects exist but are 
not included (Anselin, 1988; Elhorst, 2003; Debarsy and Ertur, 2010), it is also likely that the omission of spatial effects may have played a role.

The estimated speed of convergence becomes much higher when regionspecific fixed effects are taken into account as reported in the second column of Table 2.3. This high convergence speed of $20 \%$ corresponds to a half life of about only 3.5 years. Therefore, when conditioned on timeinvariant region specific factors, we observe a conditional convergence speed that is considerably different than the earlier estimated absolute convergence speeds.

The remaining columns of Table 2.3 report the estimation results with spatial effects. For a better elaboration on the results of these models, we draw attention to part (b) of Table 2.6, where the LR and Wald test p-values for the Base (FE), SAR, and SEM models (with the unrestricted model being the GSM) are presented. The test results suggest that the spatial lag parameter $\rho$ is not significantly different than zero in the GSM, and that a SEM specification is valid. In other words, the test results suggest that the GSM cannot collapse to a SAR specification where $\lambda \neq 0$, or to the base model where both $\rho$ and $\lambda$ are zero.

The preferred SEM model, which controls for the region specific constant effects, finds evidence for conditional convergence (a negative and significant $\beta$ ). This model also yields a convergence speed of almost $20 \%$ and a half life of 3.5 years similar to the base model results. These estimates of convergence speeds are much higher than the "legendary $2 \%$ " reported in the literature which is mostly based on national economies (Durlauf et al., 2005; De Groot and Florax, 2005). We also observe in the SEM results a significant $\lambda$ which suggests that spatial correlation exists among omitted terms.

Table 2.4 presents the estimation results when the term including the explanatory variables $\left(\sum_{k=1}^{m} \gamma_{k} x_{k, i t}\right)$ is included. The results are very similar in all models; all four specifications find conditional convergence, and estimate that internet infrastructure has a positive and significant effect on regional per capita income. Regarding how fast this convergence process is, the interaction term $\ln y_{i, t-1} \times \ln c_{i t}$ necessitates that different 
values for the convergence factor $\beta$, the convergence speed, and the halflife should be estimated based on the level of internet infrastructure region $i$ has. As the P-values of LR and Wald tests (where the GSM is the unrestricted model) reported in part (c) of Table 2.6 suggest that spatial effects do not play a significant role when the set of explanatory variables is included, we use the results of the base model for elaborating on how fast the convergence process is. The implied values by the base model are presented for several percentiles of $c_{i t}$ in Table 2.5.

We observe that the speed of convergence increases in $c_{i t}$. For instance, regions with internet infrastructure in the fifth and ninety-fifth percentiles have about 10 percentage points of difference in the speed of convergence. This corresponds to a decrease of about one-thirds in the estimated half-life (from around four years to around 2.5 years). Therefore a regional economy benefits from internet infrastructure not only in terms of growth in per capita income, but also through a higher speed of convergence towards its steady state. Moreover, considering that the half-lives in the interquartile range reported in the third column of Table 2.5 are below the half-life estimated by the base model without the set of explanatory variables (about 3.5 in Table 2.3), we suggest that regional infrastructure including internet infrastructure helps the individual steady-states of different regions to become more homogeneous, allowing for convergence towards similar per capita income levels. As a result, internet infrastructure can be seen as providing benefits to a regional economy through three different mechanisms: growth, faster convergence to the steady state, and the homogenization of region-specific steady-states.

Another important result is the additional finding in our study is that air transport capacity is also an important regional attribute that contributes to a regional economy: all models in Table 2.4 find a positive and significant effect of air transport infrastructure $\left(\ln a_{i t}\right)$ on $\ln y_{i t}$. In relation to this result, Chapter 4 finds that regional air transport capacity has enhanced the international export performances of regions in Turkey for the period 2002-2010. Therefore, it may be possible that the observed effect of this variable is due to its contribution to better trade connections with the international markets. 
Finally, we do not observe any significant result for the land infrastructure index, $r_{i t}$. One interpretation could be that regions rely more on other means of transportation rather than land routes. It is also possible that this variable simply does not provide enough variation. Moreover, as pointed out in Chapter 5, it common to observe decreases in road lengths within Turkish regions which correspond to improvements in road infrastructure and travel times. This is a general problem whenever road stock is used as an indicator of land infrastructure in empirical research. However, measures on the quality and efficiency of roads in a regional scale do not exist for Turkey. 
TABLE 2.2

Cross-SeCtional estimation RESUlts

\begin{tabular}{lcccc}
\hline \hline & $(1)$ & $(2)$ & $(3)$ & $(4)$ \\
& Base Model & SAR & SEM & GSM \\
\hline ln $y_{0}$ & $0.865^{* * *}$ & $0.744^{* * *}$ & $0.907^{* * *}$ & $0.769^{* * *}$ \\
& $(0.0472)$ & $(0.0695)$ & $(0.0253)$ & $(0.0714)$ \\
$\alpha$ & $1.322^{* * *}$ & -0.563 & $1.047^{* * *}$ & -0.334 \\
& $(0.318)$ & $(0.890)$ & $(0.167)$ & $(0.741)$ \\
\hline$\beta$ & $-0.135^{* * *}$ & $-0.256^{* * *}$ & $-0.0934^{* * *}$ & $-0.231^{* * *}$ \\
& $(0.0472)$ & $(0.0695)$ & $(0.0253)$ & $(0.0714)$ \\
$\rho$ & & $0.381^{* *}$ & & $0.325^{* *}$ \\
$\lambda$ & & $(0.174)$ & & $(0.164)$ \\
& & & $-1.403^{* *}$ & -0.856 \\
& & & $(0.596)$ & $(0.757)$ \\
\hline Convergence speed & 0.0121 & 0.0246 & 0.00817 & 0.0219 \\
Half-life & 57.23 & 28.16 & 84.87 & 31.62 \\
Observations & 26 & 26 & 26 & 26 \\
Log-likelihood & 25.55 & 28.41 & 27.38 & 29.06 \\
AIC & -47.11 & -48.83 & -46.76 & -48.11 \\
BIC & -44.59 & -43.79 & -41.73 & -41.82 \\
\hline
\end{tabular}

Stata module for spatial models: XSMLE (see footnote 30 ).

SAR: Spatial Autoregressive Model.

SEM: Spatial Error Model.

GSM: General Spatial Model.

Standard errors in parentheses: ${ }^{*} p<0.10,{ }^{* *} p<0.05,{ }^{* * *} p<0.01$ 
TABLE 2.3

PANEL ESTIMATION RESUlts

\begin{tabular}{|c|c|c|c|c|c|}
\hline & $(1)$ & $(2)$ & $(3)$ & $(4)$ & $(5)$ \\
\hline & Base Model & Base Model & SAR & SEM & GSM \\
\hline \multirow[t]{2}{*}{$\overline{l n} y_{i, t-1}$} & $0.987^{* * *}$ & $0.819^{* * *}$ & $0.819^{* * *}$ & $0.822^{* * *}$ & $0.821^{* * *}$ \\
\hline & $(0.00503)$ & $(0.0397)$ & $(0.0325)$ & $(0.0322)$ & $(0.0325)$ \\
\hline \multirow[t]{2}{*}{$\beta$} & $-0.0126^{* * *}$ & $-0.181^{* * *}$ & $-0.181^{* * *}$ & $-0.178^{* * *}$ & $-0.179^{* * *}$ \\
\hline & $(0.00503)$ & $(0.0397)$ & $(0.0325)$ & $(0.0322)$ & $(0.0325)$ \\
\hline \multirow[t]{2}{*}{$\rho$} & & & 0.141 & & -0.0288 \\
\hline & & & $(0.103)$ & & $(0.140)$ \\
\hline \multirow[t]{2}{*}{$\lambda$} & & & & $0.323^{* *}$ & $0.343^{* *}$ \\
\hline & & & & $(0.128)$ & $(0.157)$ \\
\hline Convergence speed & 0.0127 & 0.200 & 0.199 & 0.196 & 0.197 \\
\hline Half-life (years) & 54.79 & 3.467 & 3.482 & 3.541 & 3.520 \\
\hline Observations & 338 & 338 & 338 & 338 & 338 \\
\hline Observations per region & 13 & 13 & 13 & 13 & 13 \\
\hline Log-likelihood & 641.8 & 662.2 & 663.1 & 665.0 & 665.0 \\
\hline Fixed effects & No & Yes & Yes & Yes & Yes \\
\hline Year Dummies & Yes & Yes & Yes & Yes & Yes \\
\hline
\end{tabular}

Stata module for spatial models: XSMLE (see footnote 30 ).

SAR: Spatial Autoregressive Model.

SEM: Spatial Error Model.

GSM: General Spatial Model.

Standard errors in parentheses: ${ }^{*} p<0.10,{ }^{* *} p<0.05,{ }^{* * *} p<0.01$ 
TABLE 2.4

PANEL ESTIMATION RESUlts

\begin{tabular}{|c|c|c|c|c|}
\hline & (1) & $(2)$ & $(3)$ & $(4)$ \\
\hline & Base Model & SAR & SEM & GSM \\
\hline \multirow[t]{2}{*}{$\ln y_{i, t-1}$} & $1.002^{* * *}$ & $0.997^{* * *}$ & $1.001^{* * *}$ & $0.999^{* * *}$ \\
\hline & $(0.0702)$ & $(0.0599)$ & $(0.0601)$ & $(0.0606)$ \\
\hline \multirow[t]{2}{*}{$\ln y_{i, t-1} \times \ln c_{i t}$} & $-0.0204^{* * *}$ & $-0.0199^{* * *}$ & $-0.0201^{* * *}$ & $-0.0200^{* * *}$ \\
\hline & $(0.00565)$ & $(0.00472)$ & $(0.00476)$ & $(0.00482)$ \\
\hline \multirow[t]{2}{*}{$\ln c_{i t}$} & $0.145^{* * *}$ & $0.142^{* * *}$ & $0.143^{* * *}$ & $0.142^{* * *}$ \\
\hline & $(0.0377)$ & $(0.0312)$ & $(0.0316)$ & $(0.0319)$ \\
\hline \multirow[t]{2}{*}{$\ln a_{i t}$} & $0.0795^{* *}$ & $0.0784^{* *}$ & $0.0767^{* *}$ & $0.0768^{* *}$ \\
\hline & $(0.0343)$ & $(0.0306)$ & $(0.0308)$ & $(0.0308)$ \\
\hline \multirow[t]{2}{*}{$r_{i t}$} & -0.0178 & -0.0172 & -0.0177 & -0.0175 \\
\hline & $(0.0126)$ & $(0.0129)$ & $(0.0128)$ & $(0.0129)$ \\
\hline \multirow[t]{2}{*}{$\rho$} & & 0.0538 & & 0.0243 \\
\hline & & $(0.104)$ & & $(0.126)$ \\
\hline \multirow[t]{2}{*}{$\lambda$} & & & 0.100 & 0.0808 \\
\hline & & & $(0.154)$ & $(0.185)$ \\
\hline Observations & 338 & 338 & 338 & 338 \\
\hline Observations per region & 13 & 13 & 13 & 13 \\
\hline Log-likelihood & 681.3 & 681.4 & 681.5 & 681.5 \\
\hline Fixed effects & Yes & Yes & Yes & Yes \\
\hline Year Dummies & Yes & Yes & Yes & Yes \\
\hline
\end{tabular}

Stata module for spatial models: XSMLE (see footnote 30).

SAR: Spatial Autoregressive Model.

SEM: Spatial Error Model.

GSM: General Spatial Model.

Standard errors in parentheses: ${ }^{*} p<0.10,{ }^{* *} p<0.05,{ }^{* * *} p<0.01$ 
TABLE 2.5

Convergence FACTORS, SPEEDS, AND ASSOCIATED HALF-Lives

(BASE MODEL)

\begin{tabular}{lccc}
\hline Percentile of $c$ & Convergence factor & Convergence speed & Half-life (years) \\
\hline $1 \%$ & -0.138 & 0.148 & 4.671 \\
$5 \%$ & -0.158 & 0.172 & 4.019 \\
$25 \%$ & -0.199 & 0.222 & 3.126 \\
$50 \%$ & -0.213 & 0.240 & 2.893 \\
$75 \%$ & -0.224 & 0.254 & 2.734 \\
$95 \%$ & -0.235 & 0.268 & 2.588 \\
$99 \%$ & -0.241 & 0.276 & 2.510 \\
\hline
\end{tabular}

TABLE 2.6

MODEL COMPARISON VERSUS GSM

Base model SAR SEM $(\mathrm{FE})$

(a) Absolute convergence cross-sectional models

LR test $\mathrm{p}$-value

$\begin{array}{lll}0.030 & 0.256 & 0.067\end{array}$

Wald test p-value

$\begin{array}{lll}0.020 & 0.258 & 0.047\end{array}$

(b) Absolute convergence panel models

LR test $\mathrm{p}$-value

$\begin{array}{lll}0.059 & 0.049 & 0.836\end{array}$

Wald test p-value

$\begin{array}{lll}0.035 & 0.029 & 0.837\end{array}$

(c) Conditional convergence panel models

LR test $\mathrm{p}$-value

$\begin{array}{lll}0.799 & 0.667 & 0.848\end{array}$

Wald test $\mathrm{p}$-value

$\begin{array}{lll}0.797 & 0.662 & 0.847\end{array}$

SAR: Spatial Autoregressive Model.

SEM: Spatial Error Model. 


\subsection{Concluding Remarks}

Internet infrastructure is arguably a component which has become more important than the other types of telecommunication infrastructure in the last two decades. The purpose of this study was to examine its role in regional per-capita income convergence. Our approach brings together the convergence theories and the theories regarding the diffusion of information in the context of economic geography. We have examined the process of $\sigma$-convergence, the spatial differences in regional per capita income and their growth rates in Turkey.

We have observed different results regarding absolute convergence and conditional convergence: When convergence was conditioned on region specific characteristics, the convergence speeds were estimated to be much higher, and the half-lives were found to be much shorter compared to when these factors were not taken into account. As a result, we have observed evidence for conditional $\beta$-convergence with reasonable speed among Turkish regions during the period 1999-2011.

As the descriptive analysis suggested that spatial effects may play a role in the convergence of Turkish regions, we modeled spatial dependence with alternative SAR, SEM, and GSM specifications. We found that internet infrastructure contributes to a regional economy in three ways: (1) by positively impacting on per-capita income, (2) by increasing the speed of convergence of a region to its steady-state, and (3) by contributing to make region-specific steady-states more alike. Therefore, as we observe that internet infrastructure can reduce the time needed for regions to converge to their steady-states, we suggest that investing in this type of infrastructure in lagging regions is important for regional convergence.

While regional time-invariant structural characteristics are controlled through the use of fixed effects, two other connectivity-enhancing variables aside of internet infrastructure were considered (air and land infrastructure). Air transport capacity was also found to play a contributing role to a regional economy. 
Finally, our analysis suggests that the economic geography of Turkey is defined by a strong core-periphery pattern and a significant clustering of regions that have lower than average income per capita levels. However, it is therefore remarkable that controlling for spatial effects did not change any of our main findings regarding the convergence process. 


\section{A Appendix}

TABLE 2.A.1

EXAMPLES OF VARIABLES USED IN THE ANALYSIS OF CONVERGENCE

\begin{tabular}{|c|c|c|c|c|}
\hline Author & Title & Sample & Period & Explanatory factors \\
\hline $\begin{array}{l}\text { Barro et al. } \\
(1991)\end{array}$ & $\begin{array}{l}\text { Convergence across states } \\
\text { and regions }\end{array}$ & $\begin{array}{l}\text { US states } \\
\text { and regions, } \\
\text { European } \\
\text { regions }\end{array}$ & $\begin{array}{l}1880-1988 \\
\text { for US, } \\
1950-1985 \\
\text { for Europe }\end{array}$ & $\begin{array}{l}\text { Regional or country dum- } \\
\text { mies, Sectoral dummies. }\end{array}$ \\
\hline $\begin{array}{l}\text { Barro and Sala-i } \\
\text { Martin (1992) }\end{array}$ & Convergence & 48 US states & $1840-1988$ & $\begin{array}{l}\text { Regional dummies, sec- } \\
\text { toral composition, educa- } \\
\text { tion, Gov't expenditure, } \\
\text { revolutions and coups, as- } \\
\text { sasinations, market distor- } \\
\text { tions. }\end{array}$ \\
\hline $\begin{array}{l}\text { Mankiw et al. } \\
(1992)\end{array}$ & $\begin{array}{l}\text { A Contribution to the Em- } \\
\text { pirics of Economic Growth }\end{array}$ & 98 countries & $1960-1985$ & $\begin{array}{l}\text { Population, investment, } \\
\text { education. }\end{array}$ \\
\hline $\begin{array}{l}\text { Sala-i Martin } \\
(1996 b)\end{array}$ & $\begin{array}{l}\text { Regional cohesion: evi- } \\
\text { dence and theories of re- } \\
\text { gional growth and conver- } \\
\text { gence }\end{array}$ & $\begin{array}{l}\text { US, Canada, } \\
\text { Japan, } 5 \\
\text { European } \\
\text { nations }\end{array}$ & $1880-1991$ & $\begin{array}{l}\text { Regional dummies, sec- } \\
\text { toral variables. }\end{array}$ \\
\hline Persson (1997) & 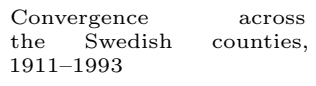 & $\begin{array}{l}\text { Swedish } \\
\text { counties }\end{array}$ & 1911-1993 & $\begin{array}{l}\text { Size of the agricultural } \\
\text { sector, migration, housing } \\
\text { rent. }\end{array}$ \\
\hline $\begin{array}{l}\text { Lall and Yilmaz } \\
\text { (2001) }\end{array}$ & $\begin{array}{l}\text { Regional economic conver- } \\
\text { gence: Do policy instru- } \\
\text { ments make a difference? }\end{array}$ & US states & 1969-1995 & $\begin{array}{l}\text { Spatial effects, public cap- } \\
\text { ital, education, time dum- } \\
\text { mies, state dummies. }\end{array}$ \\
\hline $\begin{array}{l}\text { Badinger et al. } \\
(2004)\end{array}$ & $\begin{array}{l}\text { Regional Convergence in } \\
\text { the European Union, 1985- } \\
\text { 1999: A Spatial Dynamic } \\
\text { Panel Analysis }\end{array}$ & $\begin{array}{l}196 \quad \text { Euro- } \\
\text { pean regions }\end{array}$ & 1985-1999 & $\begin{array}{l}\text { Spatial effects, invest- } \\
\text { ments, population, re- } \\
\text { gional dummies, time } \\
\text { dummies. }\end{array}$ \\
\hline $\begin{array}{l}\text { Leon-Gonzalez } \\
\text { and Montolio } \\
\text { (2004) }\end{array}$ & $\begin{array}{l}\text { Growth, convergence and } \\
\text { public investment. A } \\
\text { Bayesian model averaging } \\
\text { approach }\end{array}$ & $\begin{array}{l}\text { Spanish } \\
\text { provinces }\end{array}$ & 1965-1995 & $\begin{array}{l}\text { Private investments, pub- } \\
\text { lic investments, education, } \\
\text { sectoral structure, area, lo- } \\
\text { calization, fertility, fixed } \\
\text { regional effects. }\end{array}$ \\
\hline Arbia (2006) & $\begin{array}{l}\text { Spatial econometrics: sta- } \\
\text { tistical foundations and ap- } \\
\text { plications to regional con- } \\
\text { vergence }\end{array}$ & $\begin{array}{l}92 \quad \text { Italian } \\
\text { provinces }\end{array}$ & 1950-1999 & Spatial effects. \\
\hline Arbia (2006) & $\begin{array}{l}\text { Spatial econometrics: sta- } \\
\text { tistical foundations and ap- } \\
\text { plications to regional con- } \\
\text { vergence }\end{array}$ & $\begin{array}{l}129 \quad \text { Euro- } \\
\text { pean regions }\end{array}$ & 1950-1999 & Spatial effects. \\
\hline $\begin{array}{l}\text { Ertur and Koch } \\
(2007)\end{array}$ & $\begin{array}{l}\text { Growth, technological in- } \\
\text { terdependence and spatial } \\
\text { externalities: theory and } \\
\text { evidence }\end{array}$ & 91 Countries & $1960-1995$ & $\begin{array}{l}\text { Spatial effects, population, } \\
\text { investments. }\end{array}$ \\
\hline $\begin{array}{l}\text { Kirdar and } \\
\text { Saracoglu (2007) }\end{array}$ & $\begin{array}{l}\text { Migration and regional } \\
\text { convergence: an empirical } \\
\text { investigation for Turkey }\end{array}$ & $\begin{array}{l}67 \quad \text { Turkish } \\
\text { provinces }\end{array}$ & $1975-2000$ & $\begin{array}{l}\text { Migration, population, } \\
\text { state of emergency status. }\end{array}$ \\
\hline
\end{tabular}


TABLE 2.A.1

EXAMPLES OF VARIABLE USED IN THE ANALYSIS OF CONVERGENCE (CONT'D)

\begin{tabular}{|c|c|c|c|c|}
\hline Author & Title & Sample & Period & $\begin{array}{l}\text { Explanatory factors fac- } \\
\text { tors }\end{array}$ \\
\hline $\begin{array}{l}\text { Battisti and } \\
\text { De Vaio (2008) }\end{array}$ & $\begin{array}{l}\text { A spatially filtered mix- } \\
\text { ture of } \beta \text {-convergence re- } \\
\text { gressions for EU regions, } \\
1980-2002\end{array}$ & $\begin{array}{l}190 \text { and } 242 \\
\text { EU regions }\end{array}$ & $1980-2002$ & Spatial effects. \\
\hline Ding et al. (2008) & $\begin{array}{l}\text { Telecommunications in- } \\
\text { frastructure and regional } \\
\text { income convergence in } \\
\text { China: panel data ap- } \\
\text { proaches }\end{array}$ & $\begin{array}{l}29 \text { Chinese } \\
\text { regions }\end{array}$ & $1986-2002$ & $\begin{array}{l}\text { Spatial effects, lagged de- } \\
\text { pendent variable, invest- } \\
\text { ments, population, em- } \\
\text { ployment, telecommunica- } \\
\text { tion, urbanization, pub- } \\
\text { lic industrial output ra- } \\
\text { tio, transportation infras- } \\
\text { tructure, region and time } \\
\text { dummies. }\end{array}$ \\
\hline $\begin{array}{l}\text { Yildirim et al. } \\
(2009)\end{array}$ & $\begin{array}{l}\text { Income Inequality and } \\
\text { Economic Convergence in } \\
\text { Turkey: A Spatial Effect } \\
\text { Analysis }\end{array}$ & $\begin{array}{l}67 \quad \text { Turkish } \\
\text { provinces }\end{array}$ & $1987-2001$ & $\begin{array}{l}\text { Spatial effects, education, } \\
\text { fertility, gov't expenditure, } \\
\text { unemployment. }\end{array}$ \\
\hline $\begin{array}{l}\text { Del Bo et al. } \\
(2010)\end{array}$ & $\begin{array}{l}\text { Regional Infrastructure } \\
\text { and Convergence: Growth } \\
\text { Implications in a Spatial } \\
\text { Framework }\end{array}$ & EU regions & 1995-2006 & $\begin{array}{l}\text { Spatial effects, transporta- } \\
\text { tion infrastructure, com- } \\
\text { munication infrastructure, } \\
\text { capital stock, employment, } \\
\text { human capital, regional } \\
\text { and time fixed effects. }\end{array}$ \\
\hline $\begin{array}{l}\text { Onder et al. } \\
(2010)\end{array}$ & $\begin{array}{l}\text { The Impact of Public Capi- } \\
\text { tal Stock on Regional Con- } \\
\text { vergence in Turkey }\end{array}$ & $\begin{array}{l}26 \quad \text { Turkish } \\
\text { regions }\end{array}$ & $1980-2001$ & $\begin{array}{l}\text { Transportation public cap- } \\
\text { ital stock. }\end{array}$ \\
\hline $\begin{array}{l}\text { Pfaffermayr } \\
(2012)\end{array}$ & $\begin{array}{l}\text { Spatial convergence of re- } \\
\text { gions revisited: a spatial } \\
\text { maximum likelihood panel } \\
\text { approach }\end{array}$ & $\begin{array}{l}\text { European re- } \\
\text { gions }\end{array}$ & $1980-2005$ & $\begin{array}{l}\text { Spatial effects, country } \\
\text { and time dummies. }\end{array}$ \\
\hline
\end{tabular}




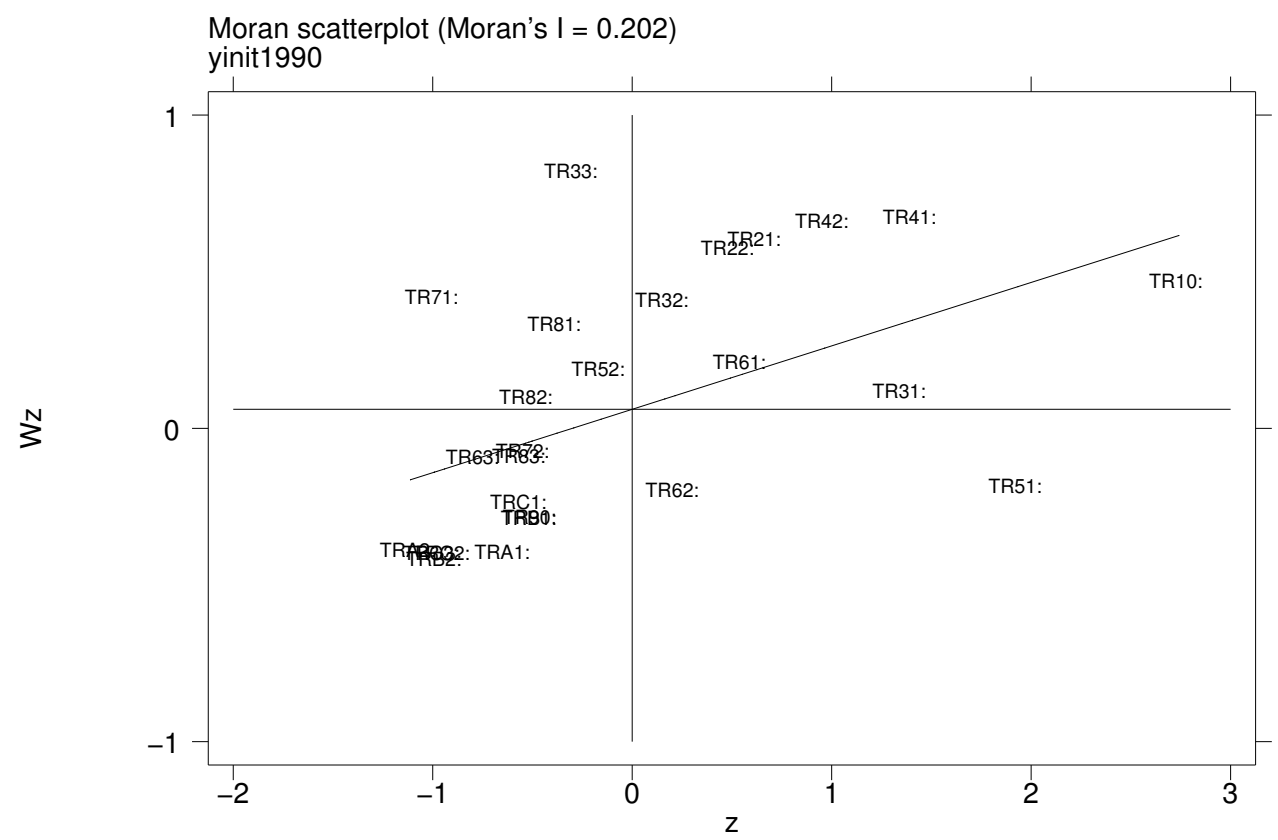

FIGURE 2.A.1

Moran scatterplot: Per Capita GVA, 1990 (Moran's I: 0.249). 


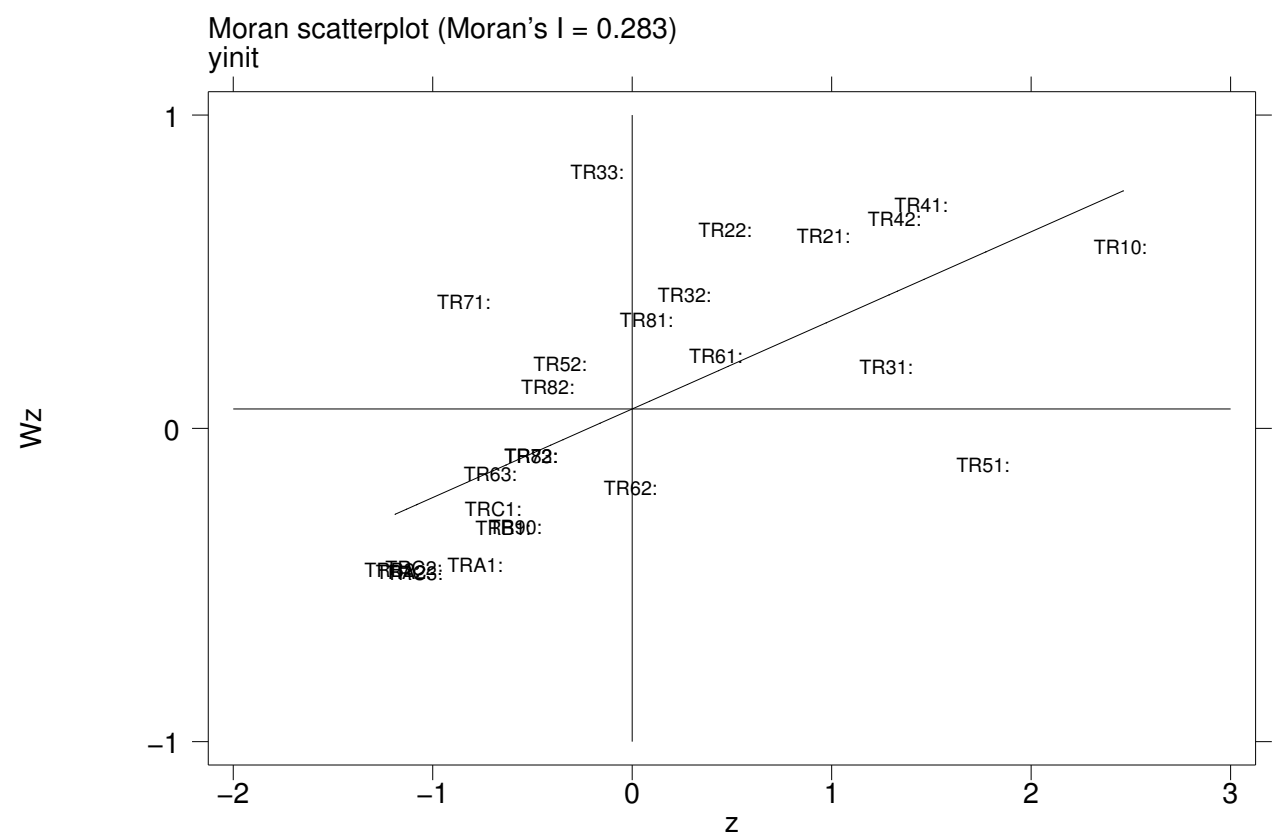

FiguRE 2.A.2

Moran scatterplot: Per Capita GVA, 1999 (Moran's I: 0.332). 


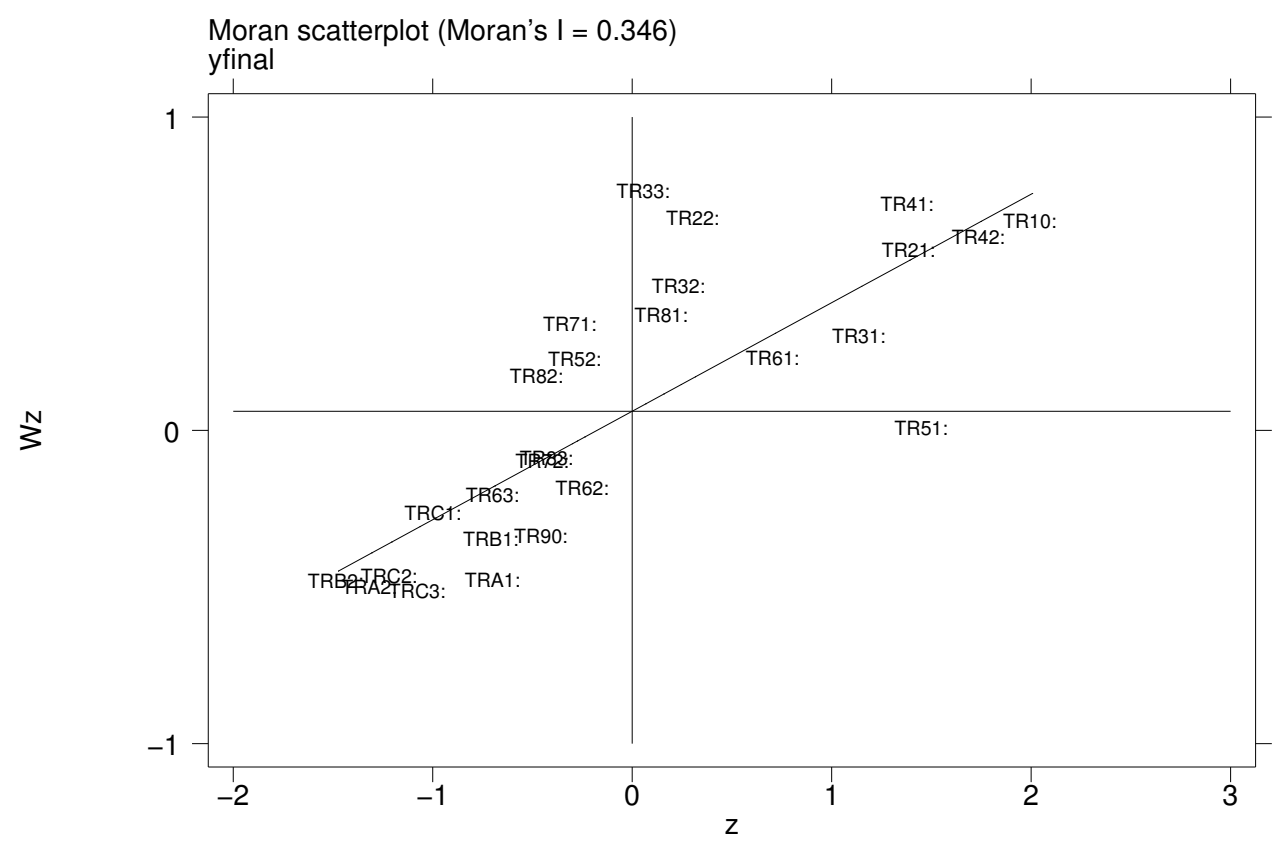

Figure 2.A.3

Moran scatterplot: Per Capita GVA, 2011 (Moran's I: 0.353). 


\section{CHAPTER 3}

\section{Infrastructure and Trade: A Meta-Analysis ${ }^{1}$}

\subsection{Introduction}

The export-led growth hypothesis, ${ }^{2}$ and the underlying reasons of persistent trade deficits have been highly researched and debated by academics and policymakers. Within the context of free trade, ways to increase competitiveness other than through exchange rate interventions, tariffs,

\footnotetext{
${ }^{1}$ This chapter is the result of a joint work with Peter Nijkamp and Jacques Poot. The title and content of this chapter is identical to its published version in REGION - the journal of the European Regional Science Association (Volume 1, Number 1, 2014, 25-65), co-authored by Peter Nijkamp and Jacques Poot. The study was also presented under different titles at the 9th World Congress of Regional Science Association International; Timisoara, Romania, May 9-11, 2012; 12th PRSCO Summer Institute and the 4th International Conference of RSAI on Regional Science and Sustainable Regional Development, Renmin University, Beijing, China, July 3-6, 2012; Meta-analysis in Economics Research Network (MAER-Net) Colloquium, Edith Cowan University, Perth, Australia, September 18-20, 2012; New Zealand Productivity Commission, Wellington, New Zealand, September 28, 2012; Tinbergen Institute, Amsterdam, The Netherlands, November 26, 2012; Economics Department, University of Canterbury, Christchurch, New Zealand, May 24, 2013. Other versions of this manuscript are also available online as a UNU-MERIT working paper with serial number 2013-032, a Vrije Universiteit Amsterdam research memorandum available at http://dare.ubvu.vu.nl/handle/1871/48101, and as an online document at http://www.econ.canterbury.ac.nz/research/pdf/CNP\%20paper\% 20UoC\%20May\%202013.pdf.

${ }^{2}$ The export-led growth hypothesis argues that the growth of exports stimulates an economy through technological spillovers and other externalities (Marin, 1992)
} 
and quotas have been attracting interest. The reduction of transport costs is arguably the most emphasized such method. Formally, transport costs are seen as a determining factor of trade flows in the gravity model of trade. Regarding this relationship between transport costs and trade, Volpe Martincus et al. (2014, p.149) state "the extent to which these costs matter is, however, far less well-established." As a result, with respect to transport costs, the effects of trade-related infrastructure on trade flows have been increasingly become a focal point in studies examining the trade performance of countries and regions in recent years.

The present study uses meta-analysis and meta-regression techniques to synthesize various "quantitative opinions" (Poot, 2014) that can be found in this literature. The type of infrastructure that we focus on is mainly public infrastructure in transportation and communication. Our meta-analysis has several objectives. First, because all estimated effects are in the form of comparable elasticities, we can calculate precisionweighted averages of the likely impact of a given percentage increase in transportation infrastructure, broadly interpreted, on a country's trade. Second, we show that this likely impact is larger in developing countries and is expected to be trade balance enhancing. Third, we show how such weighted average estimates from the literature are linked to a wide range of study features. Fourth, the systematic analysis of all studies conducted to date can provide a platform for designing new primary studies. And fifth, our meta-regression analysis is more transparent and replicable than a conventional narrative literature review. The data used in this study and the Stata code can be downloaded. ${ }^{3}$

Infrastructure is a multidimensional concept that is measured in various ways: both in relation to trade performance, and in estimating its impact on growth, welfare, efficiency, and other types of economic outcomes. As will be seen in our literature survey, empirical research often defines infrastructure as a portfolio of components, meaningful only in an integrated sense. Consequently, there exists a wide range of approaches in the literature regarding the conceptualization and classification of infrastructure. Martin and Rogers (1995, p.336) define public infrastruc-

\footnotetext{
${ }^{3}$ They are available at http://merit.unu.edu/staff/celbis/.
} 
ture as "any facility, good, or institution provided by the state which facilitates the juncture between production and consumption. Under this interpretation, not only transport and telecommunications but also such things as law and order qualify as public infrastructure." In this study, we focus exclusively on models that estimate the impacts of indicators of transportation and communication infrastructure. Recognizing the "collective" nature of infrastructure, we pay specific attention to variation in effect size in terms of the way in which infrastructure is measured in the primary studies. Nonetheless, the remaining types of public infrastructure such as rule of law, regulatory quality, etc. are to some extent considered by controlling for such attributes in the meta-regression models employed in this study.

We collected a large number of research articles that use regression analysis with at least one transportation and/or communication infrastructurerelated factor among the explanatory variables, and a dependent variable that represents either export or import volumes or sales. These papers have been collected by means of academic search engines and citation tracking. Our search yielded 36 articles published between 1999 and 2012, which provided sufficiently compatible information for meta-analytical methods. These papers are broadly representative of the literature in this area. Section 5 describes the selection of primary studies and coding of data.

The rest of this paper is structured as follows. Section 3.2 provides a short narrative literature survey. The theoretical model that underlies most regression models of merchandise trade flows and the implications for meta-regression modeling are outlined in Section 3.3. The metaanalytic methodology is briefly described in Section 3.4. The data are discussed in Section 3.5, which is followed by descriptive analysis in Section 3.6, and meta-regression modeling in Section 3.7. Section 3.8 presents some final remarks. 


\subsection{Literature Review}

The broad literature on infrastructure and trade provides certain stylized facts: the relative locations of trade partners and the positioning of infrastructure, together with the trajectories of trade, can be seen as integral features that play a role in the relationship between infrastructure and trade flows. The location of physical infrastructure and the direction of trade strongly imply a spatial dimension to the relationship and can be subject to various costs that are closely linked with space, infrastructure quality and availability. Thus, the relationship in question is usually assessed in relation to space and trade costs. For instance, Donaghy (2009, p.66) states that "trade, international or interregional, is essentially the exchange of goods and services over space. By definition, then, it involves transportation and, hence, some transaction costs." The analysis of the impact of transport costs on trade has a long history starting with von Thünen (1826), and later elaborated by Samuelson $(1952,1954)$ Mundell (1957); Geraci and Prewo (1977); Casas (1983); Bergstrand (1985) and others. Recently, the specific role of infrastructure in trade has been attracting increasing attention. The relationship has become more prominent in the trade literature especially after seminal studies such as Bougheas et al. (1999) and Limao and Venables (2001), who empirically demonstrate that infrastructure plays an important role in determining transport costs.

Nevertheless, pinpointing the exact impact of infrastructure on trade remains a challenge. The range of estimates found in the literature is wide. This may be due to numerous factors such as the relevant geographical characteristics, interrelations of different infrastructure types, infrastructure capacity utilization, and study characteristics. Additionally there are challenges in the ways in which infrastructure is defined. Bouet et al. (2008, p.2) draw attention to this by stating:

"Quantifying the true impact of infrastructure on trade however is difficult mainly because of the interactive nature of different types of infrastructure. Thus, the impact of greater 
telephone connectivity depends upon the supporting road infrastructure and vice versa. Most importantly, the precise way this dependence among infrastructure types occurs is unknown and there does not exist any a priori theoretical basis for presuming the functional forms for such interactions."

Thus, the infrastructure effects may be non-linear and may need to be explored by taking account of the interactions of different infrastructure types. Additionally, Portugal-Perez and Wilson (2012a) draw attention to the possibility of infrastructure satiation in their results from a sample of 101 countries. They find that the impact of infrastructure enhancements on export performance is decreasing in per capita income while information and communication technology is increasingly influential for wealthier countries, implying diminishing returns to transport infrastructure.

Another question that arises in assessing the impact of infrastructure on trade is the asymmetry in the impact of infrastructure in the two directions of bilateral trade. In this regard, Martínez-Zarzoso and NowakLehmann (2003) examine the EU-Mercosur bilateral trade flows and conclude that investing in a trade partner's infrastructure is not beneficial because only the exporter's infrastructure enhances trade. This result is not universal, however. Limao and Venables (2001) consider importer, exporter, and transit countries' levels of infrastructure separately and conclude that each of these dimensions of infrastructure positively impact bilateral trade flows. Similarly, Grigoriou (2007) concludes that - based on results obtained from a sample of 167 countries - road construction within a landlocked country may not be adequate to enhance trade since transit country infrastructure, bargaining power with transit countries, and transport costs also play important roles in trade performance.

Additionally, the impact of infrastructure may not be symmetric for trade partners who have different economic characteristics. For example, Longo and Sekkat (2004) find that both exporter and importer infrastructure play a significant role in intra-African trade. However, these authors do not find a significant infrastructure impact regarding trade flows 
between Africa and major developed economies. In another study on intra-African trade, Njinkeu et al. (2008) conclude that port and services infrastructure enhancement seem to be a more useful tool in improving trade in this region than other measures.

Another issue is that infrastructure specific to one geographical part of an economy may affect exports or imports at another location within the same economy. If the two locations are relatively far apart, this may yield unreliable results when broad regions are the spatial unit of measurement. Smaller spatial units of analysis may then be beneficial; however, subnational-level studies on the impact of infrastructure on trade are relatively rare. $\mathrm{Wu}$ (2007) provides evidence from Chinese regions and finds a positive impact of infrastructure (measured as total length of highways per square kilometer of regional area) on export performance. Similarly, in another sub-national level study, Granato (2008) examines the export performance of Argentinean regions to 23 partner countries. The author finds that transport costs and regional infrastructure are important determinants of regional export performance.

In the trade literature, infrastructure is usually measured in terms of stock or density, or by constructing a composite index using data on different infrastructure types. Adopting a broad view of infrastructure, Biehl (1986) distinguishes the following infrastructure categories: transportation, communication, energy supply, water supply, environment, education, health, special urban amenities, sports and tourist facilities, social amenities, cultural amenities, and natural environment. The transportation category can be classified into subcategories such as roads, railroads, waterways, airports, harbors, information transmission, and pipelines (Bruinsma et al., 1989). Nijkamp (1986) identifies the features that distinguish infrastructure from other regional potentiality factors (such as natural resource availability, locational conditions, sectoral composition, international linkages and existing capital stock) as high degrees of: publicness, spatial immobility, indivisibility, non-substitutability, and monovalence. Based on the methods employed in the primary studies, we distinguish two main approaches regarding the measurement of infrastructure: the usage of variables measuring specific infrastructure types, and/or employing infrastructure indexes. This point is further 
elaborated in Section 3.5.

\subsection{The Theory of Modeling Trade Flows}

An improvement in infrastructure is expected to lower the trade hindering impact of transport costs. Transport costs have a negative impact on trade volumes as trade takes place over space, which incurs costs in moving products from one point to another. Such costs may include fuel consumption, tariffs, rental rates of transport equipment, public infrastructure tolls, and time costs. A convenient way to represent such costs is the "iceberg melting" model of Samuelson (1954) in which only fractions of goods that are shipped arrive at their destination. In this regard, Fujita et al. (1999) refer to von Thunen's example of trade costs where a portion of grain that is transported is consumed by the horses that pull the grain wagon. Fujita et al. (1999) model the role of such trade costs in a world with a finite number of discrete locations where each variety of a product is produced in only one location and all varieties produced within a location have the same technology and price. The authors show that the total sales of a variety particular to a specific region depends - besides factors such as the income levels in each destination and the supply price - on the transportation costs to all destinations.

Anderson and van Wincoop (2003) show that bilateral trade flows between two spatial trading units depend on the trade barriers that exist between these two traders and all their other trade partners. The authors start with maximizing the CES utility function:

$$
\left(\sum_{i=1}^{N} \beta_{i}^{(1-\sigma) / \sigma} c_{i j}^{(\sigma-1) / \sigma}\right)^{\sigma /(\sigma-1)}
$$

with substitution elasticity $\sigma>1$ and subject to the budget constraint 


$$
\sum_{i=1}^{N} p_{i j} c_{i j}=y_{j}
$$

where subscripts $i$ and $j$ refer to regions (economies) and each region is specialized in producing only one good. $N$ is the number of regions. $c_{i j}$ is the consumption of the goods from region $i$ by the consumers in region $j, \beta_{i}$ is a positive distribution parameter, and $y_{j}$ is the size of the economy of region $j$ in terms of its nominal income. $p_{i j}$ is the cost, insurance and freight (cif) price of the goods from region $i$ for the consumers in region $j$ and is equal to $p_{i} t_{i j}$ where $p_{i}$ is the price of the goods of region $i$ in the origin (supply price) and $t_{i j}$ is the trade cost factor between the origin $i$ and the destination $j$, and $p_{i j} c_{i j}=x_{i j}$ is the nominal value of exports from $i$ to $j$ For the case of intra-economy trade (i.e. trade from $i$ to $i$ ) $t_{i i}$ is taken as 1, implying frictionless trade. The income of region $i$ is the sum of the values of all exports of $i$ to the other regions:

$$
y_{i}=\sum_{j=1}^{N} x_{i j}
$$

Maximizing (3.1) subject to (3.2), imposing the market clearing condition (3.3), and assuming that $t_{i j}=t_{j i}$ (i.e. trade barriers are symmetric) leads to the gravity equation:

$$
x_{i j}=\frac{y_{i} y_{j}}{y^{W}}\left(\frac{t_{i j}}{P_{i} P_{j}}\right)^{1-\sigma}
$$

where $y^{W} \equiv \sum_{j} y_{j}$ is the world nominal income. Anderson and van Wincoop $(2003,2004)$ refer to $P_{i}$ and $P_{j}$ as "multilateral resistance" variables which are defined as follows: 


$$
\begin{aligned}
& P_{i}^{1-\sigma}=\sum_{j=1}^{N} P_{j}^{\sigma-1} \theta_{j} t_{i j}^{1-\sigma}, \quad \forall i \\
& P_{j}^{1-\sigma}=\sum_{i=1}^{N} P_{i}^{\sigma-1} \theta_{i} t_{i j}^{1-\sigma}, \quad \forall j
\end{aligned}
$$

in which $\theta$ is the share of region $j$ in world income, $\frac{y_{j}}{y^{W}}$. Therefore, the authors show in equations (3.5) and (3.6) that the multilateral resistance terms (MRT) depend on the bilateral trade barriers between all trade partners. Moreover, the gravity equation (4.5) implies that the trade between $i$ and $j$ depends on their bilateral trade barriers relative to the average trade barriers between these economies and all their trading partners. Anderson and van Wincoop (2003) finalize their development of the above gravity model by defining the trade cost factor as a function of bilateral distance $\left(d_{i j}\right)$ and the presence of international borders. Here, $t_{i j}=b_{i j} d_{i j}^{\rho}$; where if an international border between $i$ and $j$ does not exist $b_{i j}=1$, otherwise it is one plus the tariff rate that applies to that specific border crossing.

Infrastructures can be interpreted as the facilities and systems that influence the effective bilateral distance $\left(d_{i j}\right)$. Lower levels of infrastructural quality can increase transportation costs. Consider for example, increased shipping costs in a port when there is congestion due to insufficient space; higher fuel consumption due to low quality roads; and more time spent in transit because of shortcomings in various types of facilities. Within the context of the iceberg melting model mentioned earlier, Bougheas et al. (1999) construct a theoretical framework in which better infrastructure increases the fraction that reaches the destination through the reduction of transport costs. By including infrastructure variables in their empirical estimation using a sample of European countries, the authors find a positive relationship between trade volume and the combined level of infrastructure of the trading partners. Many other studies on bilateral trade flows have constructed specific functional forms of the 
bilateral trade barriers (trade costs) that take the level of infrastructure into account.

An important assumption in the derivation of the gravity model presented in equation (4.5) is that $t_{i j}=t_{j i}$, which leads to $x_{i j}=x_{j i}$ (balanced bilateral trade). In practice, every trade flow is directional and infrastructure conditions at the origin of trade (the exporting country) may impact differently on the trade flow than conditions at the destination of trade (the importing country). Defining $k_{i}\left(k_{j}\right)$ as the infrastructure located in origin $i$ (destination $j$ ), referred to in the remainder of the paper as "exporter infrastructure" and "importer infrastructure," this implies that $\partial d_{i j} / \partial k_{i} \neq \partial d_{i j} / \partial k_{j}$. At the same time, there are empirically two ways to measure the trade flow: as export at the point of origin or as import at the point of destination. This implies that from the perspective of any given country $i$, there are in principle four ways of measuring the impact of infrastructure on trade:

- The impact of $k_{i}$ on $x_{i j}$ (own country infrastructure on own exports)

- The impact of $k_{i}$ on $x_{j i}$ (own country infrastructure on own imports)

- The impact of $k_{j}$ on $x_{i j}$ (partner country infrastructure on own exports)

- The impact of $k_{j}$ on $x_{j i}$ (partner country infrastructure on own imports)

Logically, with a square trade matrix, $i$ and $j$, can be chosen arbitrarily and the impact of $k_{i}$ on $x_{i j}$ must therefore be the same as the impact of $k_{j}$ on $x_{j i}$ (and the impact of $k_{i}$ on $x_{j i}$ the same as the impact of $k_{j}$ on $x_{i j}$ ). Thus, in a cross-section setting, a regression of world trade on infrastructure gives only two effect sizes in theory. Such a regression equation, when estimated with bilateral trade data, may look like: $\ln \left(x_{i j}\right)=a+b_{o} \ln \left(k_{i}\right)+b_{d} \ln \left(k_{j}\right)+$ othervars $+e_{i j}$ where a is $a$ constant term, $b_{o}$ is the origin infrastructure elasticity of trade (exporter 
infrastructure), $b_{d}$ is the destination infrastructure elasticity of trade (importer infrastructure) and $e_{i j}$ is the error term. With $n$ countries, $i=1, \ldots, n$ and $j=1, \ldots, n-1$ and the number of regression observations is $n(n-1)$.

An issue that arises in practice is that regressions may yield different results when estimated with export data as compared with import data. In other words, referring to $b_{o x}$ and $b_{d x}$ as $b_{o}$ and $b_{d}$ estimated with export data (and $b_{o m}$ and $b_{d m}$ similarly defined with import data); in theory $b_{o x}=b_{o m}$ and $b_{d x}=b_{d m}$, but we shall see that in our metaregression analysis $b_{o x}>b_{o m}$, while $b_{d x}<b_{d m}$. This simply means that a larger estimate is obtained when the trade flow is defined from the perspective of the country where the infrastructure is located rather than from the perspective of the partner country. Hence, producer/exporter country infrastructure has a bigger effect when measured with export data, while consumer/importer country infrastructure has a bigger effect when measured with import data.

\subsection{Methodology}

Meta-analysis of empirical research, first defined by Glass (1976) as "the analysis of analyses" has been a common method in experimental research such as medicine and psychology since the early $20^{\text {th }}$ century and has gained popularity in economic research in recent decades (Poot, 2014; Ridhwan et al., 2010). Stanley and Jarrell (1989, p.301) state "metaanalysis is the analysis of empirical analyses that attempts to integrate and explain the literature about some specific important parameter."

Meta-analysis compares how alternative study characteristics reflect on statistical findings; in other words, it aims to explain the source of variation among empirical results (Melo et al., 2009). As in this study, it is common in meta-analytic research to take the units of observation as estimates of a given coefficient and test the null hypothesis that this elasticity is zero (Rose and Stanley, 2005). A general approach to render 
coefficients from different models and studies comparable is to represent the collected effects sizes in the form of elasticities (if they are provided as such), or to convert these effect sizes to elasticities if the primary study presents the necessary descriptive statistics to do so. A descriptive synthesis, followed by meta-regression analysis (elaborated below) would be helpful to identify the specific methodological differences leading to different results in terms of both direction and magnitude. Therefore, the researcher can gain new insight on how, for example, the inclusion of a certain variable or adoption of a different estimation strategy affects the results available in the literature. Changes in findings can also be observed with respect to samples used in the primary studies or the time periods in focus.

Results from meta-analytic research can potentially shed light on certain policy issues that require a research synthesis. Florax et al. (2002) draw attention to the area of applied, policy-related macroeconomics being quite open to the application of meta-analysis. Examples of recent applications of meta-analysis in economic policy include: Genc et al. (2012) on immigration and international trade; Cipollina and Pietrovito (2011) on trade and EU preferential agreements; Ozgen et al. (2010) on migration and income growth; Egger and Lassmann (2012) on common language and bilateral trade; Ridhwan et al. (2010) on monetary policy; De Groot et al. (2009) on externalities and urban growth; Doucouliagos and Laroche (2009) on unions and firm profits; Nijkamp and Poot (2004) on fiscal policies and growth; and Disdier and Head (2008) on the effect of distance on bilateral trade. Meta-analysis can be used to address the impact of differences between studies in terms of design of the empirical analysis, for example with respect to the choice of explanatory variables (Nijkamp et al., 2011). Fundamentally, metaanalysis allows the researcher to combine results from several studies in order to reach a general conclusion (Holmgren, 2007). In this regard, Cipollina and Salvatici (2010, p.65) state that the chief goal of metaanalysis is to test the hypothesis that the true coefficient values are zero "when the findings from this entire area of research are combined." In economics, however, the emphasis is placed on identification by means of meta-regression analysis (MRA) of a given quantitative impact, and on study characteristics that are statistically significant in explaining 
the variation in study outcomes (Poot, 2014). Meta-regression analysis can be employed to discover how much the results obtained in primary studies are influenced by methodological aspects of the research together with the geographical and temporal attributes of the data used. Since the impacts of infrastructure on trade estimated in various studies differ widely in magnitude and significance, MRA can yield important results with respect to the choice of empirical and theoretical attributes of the primary study. We use the guidelines for MRA as published in Stanley et al. (2013).

The methodology in this study can be broken into several components. We first descriptively report the observed variation in infrastructure elasticities of trade in Section 3.6. The results are reported based on several categorizations of study characteristics. Next, we employ a set of meta-regression models in Section 3.7 for a better understanding of the joint effect of the various study characteristics, while also taking possible publication bias explicitly into account. First, we briefly comment on study selection in the next section.

\subsection{Data}

The presence of at least one infrastructure-related factor among the explanatory variables in a primary study, and a dependent variable that represents export or import volumes or sales has been the main prerequisite in our data collection. Articles have been collected using the academic search engines JSTOR, EconLit, Google Scholar, SpringerLink, and Web of Science by using keywords such as "Infrastructure," "Public Capital," "Trade," "Export," "Import," "Trade Facilitation," and "Trade Costs" in various combinations. We are confident that our selected articles are the vast majority of comparable empirical studies on this topic. Studies that have not been published in English are the only obvious exception.

Numerous authors construct indexes representing the stock or level of 
infrastructure in the countries or regions that are used for primary analyses. An index can be based on a broad definition of infrastructure or on sub-categories, such as transportation or communication infrastructure. Depending on specific study attributes such as geographical coverage or spatial scale, infrastructure indexes are usually built by combining regional/national infrastructural data scaled by surface or population. Such indexes may include: road, railroad, or highway density/length, paved roads as a percentage of total road stock, number of fax machines, number of fixed and/or mobile phone line connections, number of computers, number of internet users, aircraft traffic and passengers, number of paved airports, maritime (port) traffic statistics, fleet share in the world, and electricity consumption. Some studies calculate these indexes either in a combined way for the trade partners, or separately for each partner, and sometimes also for the transit regions. For example, Bandyopadhyay (1999) uses road and railway, and phone network density separately as proxies for the technological level and the efficiency of the distribution sector. Using a sample of OECD economies, the author finds strong evidence that the distribution sector of an economy has important implications for its international trade performance.

An alternative to the index approach is the measurement of infrastructure in one or more specific ways in the statistical analysis. Focusing explicitly on railroads, phone connections, or port traffic can be examples of this approach. For example, Shepherd and Wilson (2006) focus specifically on roads and construct minimum and average road quality indexes for the trading partners. Similarly, Nordas and Piermartini (2004) also construct - in addition to considering an overall index - indexes for specific types of infrastructure and employ dummy variables in their estimation to represent infrastructure quality. These authors find a significant and positive impact of infrastructural quality on bilateral trade with port efficiency being the most influential variable in the model.

In our study, the effect size is defined as the infrastructure elasticity of trade. After selecting the studies that directly report the impact of exporter and/or importer infrastructure in comparable elasticities, and those that provided sufficient information for elasticities to be calculated, our data set consists of 542 effect sizes from 36 primary studies ranging 
from 1999 to 2012. Tables 3.1 and 3.2 describe the studies used in our analysis and report several descriptive statistics. The geographical coverage, estimation techniques, dependent variable choice (exports or imports), and the way in which infrastructure was measured are reported in Table 3.1. Table 3.2 summarizes the reported elasticities in each of the 36 studies, categorized by whether the dependent variable was exports or imports; whether the location of the infrastructure was at the point of production (exporter infrastructure); consumption (importer infrastructure); or measured as combined/transit infrastructure. Export equations yielded 307 elasticities within a considerable range of about -2 to +15 and an average value of 0.76 . Import equations yielded 235 elasticities within the range of -2 and +8 , with an average value of 0.38 . Hence, regressions using export data clearly yielded larger elasticities.

Among our sample of 36 studies, 15 appear in peer-reviewed journals, while 21 studies are published as conference, discussion, or working papers; policy documents, or book chapters. Twelve studies were published by international organizations such as the World Bank, OECD, and WTO or had at least one author affiliated with these organizations. ${ }^{4}$ First, studies that only use a combined or transit infrastructure measure for both trade partners or estimate the impact of transit infrastructure that lies between partners were dropped. Second, one effect size for which the standard error was reported as zero (which causes problems with the meta-regression) was dropped. Third, extreme outlier observations for exporter and importer infrastructure elasticities were dropped. Following this, twenty-seven studies and 379 effect sizes remain and are used for all further analyses in this paper. ${ }^{5}$ Figures 1 and 2 show the quantile plots of the effect sizes in our final data set for exporter infrastructure and importer infrastructure respectively. The ranges for the restricted data set are now similar, but a comparison of the medians and the interquartile ranges suggest a tendency for exporter infrastructure

\footnotetext{
${ }^{4}$ Hence we include in our later analysis a variable representing possible advocacy for a higher effect size for studies conducted by these organizations.

${ }^{5}$ Dropping studies that use a combined or transit infrastructure measure reduced the number of primary studies from 36 to 28 . Next, dropping extreme outliers reduced the number of studies to 27 . The extreme outliers were defined as observations that are three times the interquartile range away from the $25^{\text {th }}$ and $75^{\text {th }}$ percentiles.
} 
elasticities to be somewhat larger.

TABLE 3.1

Primary Studies InCluded in the SAMPle

\begin{tabular}{|c|c|c|c|c|}
\hline Author(s) & $\begin{array}{l}\text { Geographical } \\
\text { coverage }\end{array}$ & Methods & Trade measures & $\begin{array}{l}\text { Infrastructure } \\
\text { measurement }\end{array}$ \\
\hline $\begin{array}{l}\text { Bandyopadhyay } \\
\text { (1999) }\end{array}$ & 23 OECD countries & $\begin{array}{l}\text { OLS, IV, cross- } \\
\text { section, FE }\end{array}$ & Total Exports & $\begin{array}{l}\text { Density of road and } \\
\text { railway network. }\end{array}$ \\
\hline $\begin{array}{l}\text { Bougheas et al. } \\
(1999)\end{array}$ & $\begin{array}{l}9 \text { Core EU and Scandi- } \\
\text { navian countries }\end{array}$ & SUR, IV-SUR & Total Exports & $\begin{array}{l}\text { The product of the } \\
\text { stocks of public capi- } \\
\text { tal of exporter and im- } \\
\text { porter. }\end{array}$ \\
\hline Elbadawi (1999) & 32 Developing countries & Bilateral RE & $\begin{array}{l}\text { Manufactured } \\
\text { Exports/GDP }\end{array}$ & $\begin{array}{l}\text { Length of paved } \\
\text { roads. }\end{array}$ \\
\hline $\begin{array}{l}\text { Limao and } \\
\text { Venables (2001) }\end{array}$ & 103 World countries & Tobit, FE & Total Imports & $\begin{array}{l}\text { Index made using } \\
\text { road and rail lengths, } \\
\text { phone lines per } \\
\text { person. }\end{array}$ \\
\hline $\begin{array}{l}\text { Martínez-Zarzoso } \\
\text { and } \\
\text { Nowak-Lehmann } \\
(2003)\end{array}$ & $\begin{array}{l}\text { EU, Mercosur countries, } \\
\text { Chile ( } 20 \text { countries })\end{array}$ & $\begin{array}{l}\text { OLS, OLS on } \\
\text { means, FE, } \\
\text { RE, Dynamic } \\
\text { RE, panel } \\
\text { estimation }\end{array}$ & Total Exports & $\begin{array}{l}\text { Index made using } \\
\text { road and rail lengths, } \\
\text { phone lines per } \\
\text { person. }\end{array}$ \\
\hline $\begin{array}{l}\text { Nicoletti et al. } \\
(2003)\end{array}$ & 28 OECD countries & $\begin{array}{l}\text { Transformed } \\
\text { Least Squares, FE }\end{array}$ & Services Exports & $\begin{array}{l}\text { Length of motorways, } \\
\text { no. of aircraft depar- } \\
\text { tures. }\end{array}$ \\
\hline Raballand (2003) & $\begin{array}{l}18 \text { Land-locked and } 10 \\
\text { Island countries, } \\
18 \text { Partners }\end{array}$ & $\begin{array}{l}\text { 2SLS, regression } \\
\text { on FE's }\end{array}$ & Total Imports & $\begin{array}{l}\text { Index made of road } \\
\text { and railroad networks. }\end{array}$ \\
\hline $\begin{array}{l}\text { Jansen and } \\
\text { Nordås }(2004)\end{array}$ & 101 World countries & OLS & Total Imports & $\begin{array}{l}\text { Index of road and } \\
\text { railroad length, phone } \\
\text { lines, quality of ports, } \\
\text { density of airports. }\end{array}$ \\
\hline $\begin{array}{l}\text { Nordas and } \\
\text { Piermartini } \\
(2004)\end{array}$ & 138 World countries & OLS, FE & $\begin{array}{l}\text { Exports of } \\
\text { Various Sectors }\end{array}$ & $\begin{array}{l}\text { Index from no. of } \\
\text { airports and aircraft } \\
\text { departures, density } \\
\text { of paved roads, tele- } \\
\text { phone lines, port } \\
\text { efficiency index, me- } \\
\text { dian clearance time. }\end{array}$ \\
\hline $\begin{array}{l}\text { Wilson et al. } \\
(2004)\end{array}$ & $\begin{array}{l}75 \text { World countries } \\
\text { and sub-samples }\end{array}$ & $\begin{array}{l}\text { OLS, WLS, } \\
\text { Clustered SE's }\end{array}$ & $\begin{array}{l}\text { Manufactured } \\
\text { Exports }\end{array}$ & $\begin{array}{l}\text { Indexes from port fa- } \\
\text { cilities, inland water- } \\
\text { ways, and air trans- } \\
\text { port. }\end{array}$ \\
\hline $\begin{array}{l}\text { Brun et al. } \\
(2005)\end{array}$ & $\begin{array}{l}130 \text { World countries, } \\
\text { sub-samples }\end{array}$ & RE, IV & Total Imports & $\begin{array}{l}\text { Index made from } \\
\text { roads and railway } \\
\text { length, and no. of } \\
\text { telephone sets. }\end{array}$ \\
\hline
\end{tabular}


TABLE 3.1

PRimaRy STUdies INCLUded IN THE SAMPLE (CONT'D)

\begin{tabular}{|c|c|c|c|c|}
\hline Author(s) & $\begin{array}{l}\text { Geographical } \\
\text { coverage }\end{array}$ & Methods & Trade measures & $\begin{array}{l}\text { Infrastructure } \\
\text { measurement }\end{array}$ \\
\hline $\begin{array}{l}\text { Coulibaly and } \\
\text { Fontagné (2005) }\end{array}$ & 7 "South" countries & 2SLS, FE & Total Imports & Paved bilateral roads. \\
\hline $\begin{array}{l}\text { Marquez-Ramos } \\
\text { and } \\
\text { Martinez-Zarzoso } \\
(2005)\end{array}$ & 62 World countries & OLS, Tobit & Total Exports & $\begin{array}{l}\text { Index made of lengths } \\
\text { of various road types. }\end{array}$ \\
\hline Carrère (2006) & 130 World countries & $\begin{array}{l}\text { OLS, RE, } \\
\text { Hausman-Taylor }\end{array}$ & Total Imports & $\begin{array}{l}\text { Average road, railroad } \\
\text { and line density. }\end{array}$ \\
\hline $\begin{array}{l}\text { Elbadawi et al. } \\
(2006)\end{array}$ & 18 Developing countries & $\begin{array}{l}\text { Maximum Likeli-, } \\
\text { hood, Reduced } \\
\text { Form Tobit IV }\end{array}$ & Total Exports & Road density. \\
\hline $\begin{array}{l}\text { Fujimura and } \\
\text { Edmonds }(2006)\end{array}$ & $\begin{array}{l}6 \text { Southeast Asian } \\
\text { countries }\end{array}$ & OLS, GLS (RE) & $\begin{array}{l}\text { Major exports } \\
\text { via land/river }\end{array}$ & Road density. \\
\hline $\begin{array}{l}\text { Shepherd and } \\
\text { Wilson (2006) }\end{array}$ & $\begin{array}{l}27 \text { European and Cen } \\
\text {-tral Asian countries }\end{array}$ & $\begin{array}{l}\text { OLS, FE, RE, } \\
\text { Poisson ML, Neg. } \\
\text { Binomial Est., } \\
\text { Bootstrapped SE's }\end{array}$ & Total Exports & $\begin{array}{l}\text { Road quality index } \\
\text { between the trading } \\
\text { partners. }\end{array}$ \\
\hline De (2007) & 10 Asian countries & OLS & Total Imports & $\begin{array}{l}\text { Index from road and } \\
\text { railroad density, air } \\
\text { and port traffic, fleet } \\
\text { share in world, phone } \\
\text { lines, and electricity } \\
\text { consumption. }\end{array}$ \\
\hline $\begin{array}{l}\text { Francois and } \\
\text { Manchin (2007) }\end{array}$ & $\begin{array}{l}140 \text { World countries } \\
\text { with sub-samples }\end{array}$ & $\begin{array}{l}\text { OLS, Heckman } \\
\text { Selection, Tobit }\end{array}$ & Total Imports & $\begin{array}{l}\text { Index made of trans- } \\
\text { portation and commu- } \\
\text { nication Indicators. }\end{array}$ \\
\hline Grigoriou (2007) & 167 World countries & $\begin{array}{l}\text { GLS, FE, RE, } \\
\text { Hausman-Taylor } \\
\text { Estimator }\end{array}$ & Total Imports & $\begin{array}{l}\text { Density of the roads, } \\
\text { railroads, and no. of } \\
\text { phone lines. }\end{array}$ \\
\hline $\begin{array}{l}\text { Iwanow and } \\
\text { Kirkpatrick } \\
(2007)\end{array}$ & 78 World countries & $\begin{array}{l}\text { GLS, Heckman } \\
\text { selection }\end{array}$ & $\begin{array}{l}\text { Manufactured } \\
\text { Exports }\end{array}$ & $\begin{array}{l}\text { Index from density of } \\
\text { roads and railroads, } \\
\text { and no. of phone sub- } \\
\text { scribers. }\end{array}$ \\
\hline Persson (2007) & $\begin{array}{l}128 \text { Countries ( } 22 \mathrm{EU} \text { and } \\
106 \text { Developing countries) }\end{array}$ & Heckman Selection & Total Imports & $\begin{array}{l}\text { No. of aircraft take- } \\
\text { offs. }\end{array}$ \\
\hline $\begin{array}{l}\text { Bouet et al. } \\
(2008)\end{array}$ & $\begin{array}{l}42 \text { African countries, } \\
\text { and their trade partners }\end{array}$ & $\begin{array}{l}\text { OLS, Heckman } \\
\text { Selection, Tobit }\end{array}$ & Total Exports & $\begin{array}{l}\text { Road lengths and no. } \\
\text { of phone lines. }\end{array}$ \\
\hline $\begin{array}{l}\text { Egger and Larch } \\
(2008)\end{array}$ & 180 World Countries & $\begin{array}{l}\text { FE, Gaussian, } \\
\text { Gamma, Poisson } \\
\text { Pseudo ML, Neg. } \\
\text { Binomial Est. }\end{array}$ & Total Exports & Total road length. \\
\hline
\end{tabular}


TABLE 3.1

PRimaRy STUdies INCLUded IN THE SAMPLE (CONT'D)

\begin{tabular}{|c|c|c|c|c|}
\hline Author(s) & $\begin{array}{l}\text { Geographical } \\
\text { coverage }\end{array}$ & Methods & Trade measures & $\begin{array}{l}\text { Infrastructure } \\
\text { measurement }\end{array}$ \\
\hline Granato (2008) & $\begin{array}{l}5 \text { Argentinian regions and } \\
23 \text { trade partner countries }\end{array}$ & $\begin{array}{l}\text { OLS, Poisson } \\
\text { pseudo ML }\end{array}$ & Total Exports & $\begin{array}{l}\text { Index from road } \\
\text { length, electricity and } \\
\text { gas consumption, no. } \\
\text { of phone subscribers. }\end{array}$ \\
\hline $\begin{array}{l}\text { Kurmanalieva } \\
\text { and Parpiev } \\
(2008)\end{array}$ & 171 World Countries & $\mathrm{FE}$ & Total Imports & Road density. \\
\hline $\begin{array}{l}\text { Njinkeu et al. } \\
(2008)\end{array}$ & $\begin{array}{l}100 \text { World Countries } \\
\text { and sub-samples }\end{array}$ & OLS, FE, Tobit & $\begin{array}{l}\text { Manufactured } \\
\text { Exports }\end{array}$ & $\begin{array}{l}\text { Index made from port } \\
\text { and air transport in- } \\
\text { frastructure quality. }\end{array}$ \\
\hline $\begin{array}{l}\text { Iwanow and } \\
\text { Kirkpatrick } \\
(2009)\end{array}$ & $\begin{array}{l}124 \text { World Countries } \\
\text { and sub-samples }\end{array}$ & $\begin{array}{l}\text { GLS, Heckman } \\
\text { sample selection }\end{array}$ & $\begin{array}{l}\text { Manufactured } \\
\text { Exports }\end{array}$ & $\begin{array}{l}\text { Index made of road } \\
\text { and rail density, no. } \\
\text { of phone subscribers. }\end{array}$ \\
\hline Ninkovic (2009) & 26 Developing countries & $\mathrm{FE}, \mathrm{RE}$ & $\begin{array}{l}\text { Export share of } \\
\text { labor-intensive } \\
\text { sectors in GDP }\end{array}$ & $\begin{array}{l}\text { Road, railroad, and } \\
\text { phone line density. }\end{array}$ \\
\hline $\begin{array}{l}\text { Buys et al. } \\
(2010)\end{array}$ & 36 Sub-Saharan Countries & OLS & Total Exports & $\begin{array}{l}\text { Road quality index } \\
\text { between the trading } \\
\text { partners. }\end{array}$ \\
\hline $\begin{array}{l}\text { Hernandez and } \\
\text { Taningco }(2010)\end{array}$ & 11 East Asian Countries & OLS & $\begin{array}{l}\text { Total Imports, } \\
\text { Imports of } \\
\text { industrial supplies }\end{array}$ & $\begin{array}{l}\text { Quality of port infras- } \\
\text { tructure. }\end{array}$ \\
\hline Lawless (2010) & $\begin{array}{l}\text { Ireland and } 137 \\
\text { trade partners }\end{array}$ & OLS & Total Exports & $\begin{array}{l}\text { Density of phones and } \\
\text { computers. }\end{array}$ \\
\hline UNECA (2010) & $\begin{array}{l}52 \text { African countries } \\
\text { and } 48 \text { non-African } \\
\text { trade partners }\end{array}$ & Tobit & Total Exports & $\begin{array}{l}\text { Road and phone line } \\
\text { density. }\end{array}$ \\
\hline Dettmer (2011) & $\begin{array}{l}27 \text { OECD countries and } \\
\text { their trade partners }\end{array}$ & OLS, FE & $\begin{array}{l}\text { ICT network and } \\
\text { commercial service } \\
\text { exports }\end{array}$ & $\begin{array}{l}\text { Density of communi- } \\
\text { cation infrastructure } \\
\text { and air traffic. }\end{array}$ \\
\hline $\begin{array}{l}\text { Portugal-Perez } \\
\text { and Wilson } \\
(2012 \mathrm{a})\end{array}$ & 101 World Countries & $\begin{array}{l}\text { OLS, Heckman } \\
\text { Selection, Tobit, } \\
\text { Poisson ML }\end{array}$ & $\begin{array}{l}\text { Total Exports, } \\
\text { Exports of New } \\
\text { Goods }\end{array}$ & $\begin{array}{l}\text { Indexes from quality } \\
\text { of ports, roads, air- } \\
\text { ports, ICT indicators, } \\
\text { and railroads. }\end{array}$ \\
\hline $\begin{array}{l}\text { Vijil and Wagner } \\
(2012)\end{array}$ & 96 Developing countries & OLS, IV & $\begin{array}{l}\text { Total Exports, } \\
\text { Exports/GDP }\end{array}$ & $\begin{array}{l}\text { Index from road den- } \\
\text { sity and no. of phone } \\
\text { subscribers. }\end{array}$ \\
\hline
\end{tabular}


TABLE 3.2

DESCRIPTIVE Statistics By PRIMARY STUdY

\begin{tabular}{|c|c|c|c|c|c|c|c|c|c|}
\hline \multirow[b]{2}{*}{ Author(s) } & \multirow[b]{2}{*}{$\begin{array}{c}\text { Location of } \\
\text { Infrastructure }\end{array}$} & \multicolumn{4}{|c|}{ Export Equation } & \multicolumn{4}{|c|}{ Import Equation } \\
\hline & & Obs. & Mean & Min. & Max. & Obs. & Mean & Min. & Max. \\
\hline \multirow[t]{2}{*}{ Bandyopadhyay (1999) } & Exporter Infrastructure & 8 & 0.35 & 0.14 & 0.52 & & & & \\
\hline & Importer Infrastructure & 8 & 0.01 & -0.23 & 0.29 & & & & \\
\hline Bougheas et al. (1999) & Combined/Transit Inf. & 8 & 5.40 & 0.18 & 15.13 & & & & \\
\hline Elbadawi (1999) & Exporter Infrastructure & 4 & 0.56 & 0.46 & 0.64 & & & & \\
\hline \multirow[t]{3}{*}{ Limao and Venables (2001) } & Exporter Infrastructure & & & & & 3 & 1.10 & 1.10 & 1.11 \\
\hline & Combined/Transit Inf. & & & & & 4 & 0.64 & 0.58 & 0.77 \\
\hline & Importer Infrastructure & & & & & 4 & 1.38 & 1.32 & 1.45 \\
\hline \multirow[t]{2}{*}{ M.-Zarz. \& N.-Lehm (2003) } & Exporter Infrastructure & 13 & 0.05 & -0.02 & 0.12 & & & & \\
\hline & Importer Infrastructure & 13 & -0.05 & -0.08 & 0.01 & & & & \\
\hline Nicoletti et al. (2003) & Combined/Transit Inf. & 4 & 0.33 & 0.21 & 0.38 & & & & \\
\hline \multirow[t]{2}{*}{ Raballand (2003) } & Exporter Infrastructure & & & & & 5 & 0.22 & 0.2 & 0.24 \\
\hline & Importer Infrastructure & & & & & 5 & 0.11 & 0.09 & 0.13 \\
\hline \multirow[t]{2}{*}{ Jansen and Nordås (2004) } & Exporter Infrastructure & & & & & 3 & 0.70 & 0.67 & 0.73 \\
\hline & Importer Infrastructure & & & & & 3 & 0.45 & 0.35 & 0.55 \\
\hline \multirow[t]{2}{*}{ Nordas and Piermartini (2004) } & Exporter Infrastructure & & & & & 40 & 0.27 & -0.19 & 1.29 \\
\hline & Importer Infrastructure & & & & & 40 & 0.27 & -0.6 & 2.14 \\
\hline \multirow[t]{2}{*}{ Wilson et al. (2004) } & Exporter Infrastructure & 11 & 0.91 & 0.54 & 1.06 & & & & \\
\hline & Importer Infrastructure & 11 & 0.28 & -0.28 & 0.47 & & & & \\
\hline \multirow[t]{2}{*}{ Brun et al. (2005) } & Exporter Infrastructure & & & & & 4 & 0.40 & 0.12 & 1.18 \\
\hline & Importer Infrastructure & & & & & 4 & 0.10 & 0.06 & 0.19 \\
\hline Coulibaly \& Font. (2005) & Combined/Transit Inf. & & & & & 12 & 1.72 & 1.17 & 2.77 \\
\hline \multirow[t]{2}{*}{ M.-Ramos \& M-Zarz. (2005) } & Exporter Infrastructure & 5 & 0.53 & -0.29 & 1.38 & & & & \\
\hline & Importer Infrastructure & 5 & 0.38 & -0.47 & 1.27 & & & & \\
\hline \multirow[t]{2}{*}{ Carrère (2006) } & Exporter Infrastructure & & & & & 5 & 0.10 & 0.01 & 0.41 \\
\hline & Importer Infrastructure & & & & & 5 & 0.07 & 0.02 & 0.20 \\
\hline Elbadawi et al. (2006) & Exporter Infrastructure & 2 & 0.08 & 0.03 & 0.13 & & & & \\
\hline \multirow[t]{2}{*}{ Fujimura \& Edmonds (2006) } & Exporter Infrastructure & 10 & 0.37 & -0.66 & 1.47 & & & & \\
\hline & Importer Infrastructure & 10 & 0.3 & -1.4 & 2.15 & & & & \\
\hline Shepherd and Wilson (2006) & Combined/Transit Inf. & 32 & 0.46 & -2.09 & 1.5 & & & & \\
\hline
\end{tabular}


TABle 3.2

Descriptive Statistics by PRIMARY STUdy (CONT'D)

\begin{tabular}{|c|c|c|c|c|c|c|c|c|c|}
\hline \multirow[b]{2}{*}{ Author(s) } & \multirow[b]{2}{*}{$\begin{array}{c}\text { Location of } \\
\text { Infrastructure }\end{array}$} & \multicolumn{4}{|c|}{ Export Equation } & \multicolumn{4}{|c|}{ Import Equation } \\
\hline & & Obs. & Mean & Min. & Max. & Obs. & Mean & Min. & Max. \\
\hline \multirow[t]{2}{*}{ De (2007) } & Exporter Infrastructure & & & & & 14 & 0.13 & -0.39 & 0.40 \\
\hline & Importer Infrastructure & & & & & 14 & -0.12 & -0.49 & 0.30 \\
\hline Francois \& Manchin (2007) & Exporter Infrastructure & & & & & 38 & 0.16 & -0.01 & 1.17 \\
\hline \multirow[t]{2}{*}{ Grigoriou (2007) } & Exporter Infrastructure & & & & & 10 & 0.24 & 0.20 & 0.51 \\
\hline & Importer Infrastructure & & & & & 10 & 0.27 & 0.23 & 0.29 \\
\hline Iwan. \& Kirkpat. (2007) & Exporter Infrastructure & 11 & 1.05 & 0.68 & 1.76 & & & & \\
\hline \multirow[t]{2}{*}{ Persson (2007) } & Exporter Infrastructure & & & & & 1 & -0.07 & -0.07 & -0.07 \\
\hline & Importer Infrastructure & & & & & 1 & 0.02 & 0.02 & 0.02 \\
\hline Bouet et al. (2008) & Exporter Infrastructure & 24 & 0.24 & -1.19 & 1.61 & & & & \\
\hline Egger and Larch (2008) & Combined/Transit Inf. & 18 & 0.27 & -0.02 & 2.85 & & & & \\
\hline Granato (2008) & Exporter Infrastructure & 4 & 1.36 & 1.22 & 1.69 & & & & \\
\hline \multirow[t]{2}{*}{ Kurman. \& Parp. (2008) } & Exporter Infrastructure & & & & & 1 & 0.05 & 0.05 & 0.05 \\
\hline & Importer Infrastructure & 1 & 0.05 & 0.05 & 0.05 & & & & \\
\hline \multirow[t]{2}{*}{ Njinkeu et al. (2008) } & Exporter Infrastructure & 12 & 2.11 & 1.08 & 4.54 & & & & \\
\hline & Importer Infrastructure & 12 & 3.74 & -0.69 & 8.62 & & & & \\
\hline Iwan. \& Kirkpat. (2009) & Importer Infrastructure & 9 & 0.91 & 0.66 & 1.68 & & & & \\
\hline Ninkovic (2009) & Exporter Infrastructure & 4 & -0.02 & -0.60 & 0.34 & & & & \\
\hline Buys et al. (2010) & Exporter Infrastructure & 6 & 1.90 & 1.58 & 2.07 & & & & \\
\hline Hernand. \& Taning. (2010) & Combined/Transit Inf. & & & & & 9 & 1.69 & -2.36 & 8.10 \\
\hline Lawless (2010) & Importer Infrastructure & 8 & 0.23 & -0.17 & 0.58 & & & & \\
\hline UNECA (2010) & Exporter Infrastructure & 6 & 0.21 & 0.13 & 0.32 & & & & \\
\hline Dettmer (2011) & Combined/Transit Inf. & 20 & 0.06 & -0.11 & 0.16 & & & & \\
\hline P.-Perez \& Wilson (2012) & Exporter Infrastructure & 14 & -0.07 & -1.68 & 0.87 & & & & \\
\hline Vijil \& Wagner (2012) & Exporter Infrastructure & 14 & 1.68 & 0.47 & 2.39 & & & & \\
\hline Overall & Any Location & 307 & 0.76 & -2.09 & 15.13 & 235 & 0.38 & -2.36 & 8.1 \\
\hline
\end{tabular}


Figure 3.5.1. Quantile Plots of the Infrastructure ElasticITY OF TRADE.

(A) ExporTs

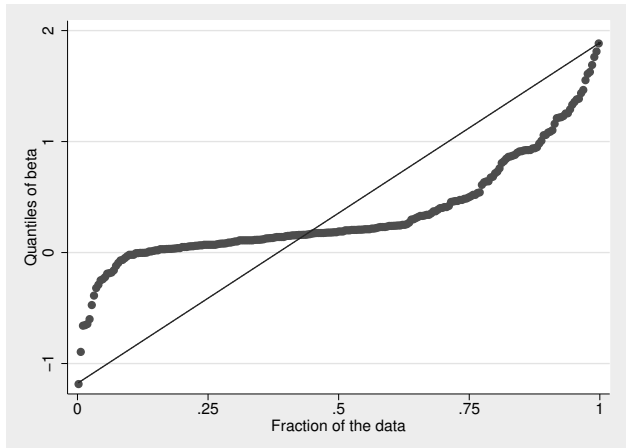

(B) IMPORTS

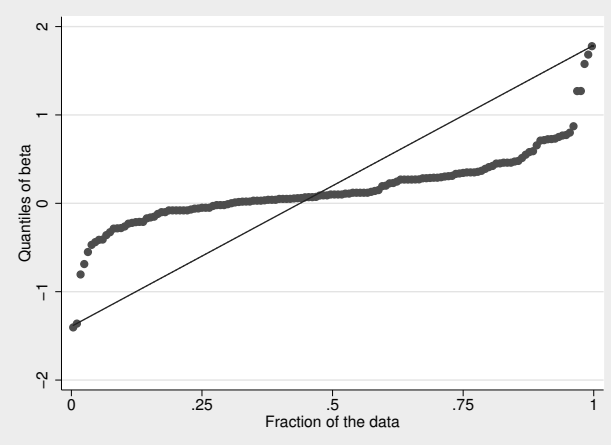

\subsection{Descriptive Analysis}

In order to conduct descriptive and regression analyses, the methodological attributes together with various other characteristics of the primary studies are coded numerically as binary variables. Definitions of the variables representing the study characteristics are provided in Table 3.3.

Overall, approximately 82 percent of the estimates in the final data set find a positive and significant infrastructure impact on trade. The descriptive statistics for all effect sizes are grouped by direction of trade, methodology, infrastructure category, development level of the relevant economies, and publication status. The results are presented in Tables 3.4 to 3.8. For ease of comparison, the combined descriptive statistics for all groups are repeated in the bottom line of each table. ${ }^{6}$

\footnotetext{
${ }^{6}$ In Table 3.5 the observations from the sub category sum to 239 rather than the total effect size number of 237 for exporter infrastructure. This is because Elbadawi et al. (2006) use Tobit and IV for the two effect sizes they estimate.
} 
Table 3.4 reinforces the earlier finding from Table 3.2 that studies where the dependent variable was exports yielded higher effect sizes on average than studies which use imports as the dependent variable. Thus, according to these raw averages, the mean effect size on exports is larger than on imports regardless of the location of infrastructure. However, irrespective of the trade data used (imports or exports), exporter infrastructure has a bigger impact than importer infrastructure, with elasticities on average 0.34 and 0.16 respectively. This implies a net gain in the balance of merchandise trade from expanding infrastructure in a particular country, an important finding which we will quantify further after controlling for study heterogeneity and publication bias. ${ }^{7}$

Nevertheless, the greater impact of exporter infrastructure is not the case across all types of estimation methods (see Table 3.5). Heckman, Tobit, and Probit estimations (that control for zero trade flows) yield larger importer infrastructure elasticities than exporter elasticities (0.49 and 0.33 respectively). When considering the type of infrastructure (see Table 3.6), a composite measure has a bigger impact than the more specific infrastructure types of land transport, maritime or air transport, and communication infrastructure. However, leaving aside the composite measure category, land transportation infrastructure appears on average, to affect trade in both directions more than the other types of infrastructure. Exporter infrastructure has again, on average, a higher effect size on trade than importer infrastructure for all categories except communication infrastructure. This is an interesting finding, as communication infrastructure has a greater impact on transaction costs than on transportation costs, because it facilitates the flow of information, which can enhance trade. It appears that communication infrastructure has a greater impact on the consumption side of the market than on the production side. Meta-regression analysis will show that this effect is

\footnotetext{
${ }^{7}$ In a general equilibrium analysis, if there are some countries that found the trade balance to improve, it must logically have deteriorated in others. Global general equilibrium gravity models that have this property are actually very rare, but see e.g. Bikker (1987). The studies in our meta-sample are all partial analyses concerned with a limited number of origin and destination countries and a rectangular rather than square trade matrix. In that case, the empirical evidence shows that, ceteris paribus, an increase in infrastructure improves the trade balance in the countries concerned.
} 
statistically significant in the model that corrects for publication bias.

In order to account for differences regarding the level of development of the economies included in the primary studies, the grouping of results is based on three types of data sets. A "Developed Economies" category is used when the author uses terms such as "Developed," "Rich," "North," "OECD," and "EU" to describe the part of the sample in which the infrastructure is located in the primary study. "Developing Economies" is used if the classification is described as "Developing," "South," or "Poor." In order to also examine the estimates obtained from samples that included both developed and developing countries, a "Mixed Samples" category was defined. Results are presented in Table 3.7. The average elasticity in mixed samples is in between those for developed countries and developing countries for exporter infrastructure. In all categories, the elasticity of exporter infrastructure is larger than that of importer infrastructure. Less developed economies seem to enjoy a higher return on infrastructure (especially if it is exporter infrastructure) compared to developed economies. This difference may be attributed to diminishing returns to investment in infrastructure capital, as is consistent with the neoclassical theory of long-run development.

In Table 3.8 we consider a measure of publication quality of the research by adopting the Australian Business Deans Council Journal Quality List ABDC (2010). "Highly Ranked Journals" refers to papers published in journals classified as A*, A, or B. "Other journals and unpublished" refers to outlets with classification $\mathrm{C}$ or D (category D includes book chapters, non-refereed working papers and conference proceedings). Exporter infrastructure has again higher average effect sizes than importer infrastructure for all categories. Moreover, studies in highly ranked journals find on average higher effect sizes for both exporter and importer infrastructure compared to other studies. In meta-analysis, this is commonly attributed to publication bias on which we elaborate further in Section 3.7.

\footnotetext{
${ }^{8}$ As classifications for some economies may change throughout the years or depending on the sources, we rely on the statement of the author(s) regarding their sample.
} 
TABle 3.3. VARiable Definitions

\begin{tabular}{l}
\hline Variable label \\
\hline Methodology \\
Model accounts for zero trade flows selection (Heckman, \\
Tobit, Probit) \\
Model accounts for endogeneity (IV-Based Estimation) \\
Gravity Model
\end{tabular}

\section{The point at which trade is measured}

Dependent variable is exports

Dependent variable is imports

\section{Infrastructure category}

Land transport infrastructure

Maritime or air transport infrastructure

Communication infrastructure

Composite measure (index)

\section{Development level of the economy}

\section{in which infrastructure is located}

Developed economy

Developing economy

Both types of economies (mixed sample)

\section{Sample structure}

Sub-national or firm level

Not cross-section

\section{Model specification}

Constrained model
Definition

Estimation is done by Heckman, Tobit, or Probit based sample selection procedures.

Estimation attempts to deal with endogeneity by using instrumental variables or lags.

The equation estimates the impact on origindestination trade flows.

The effect size is obtained from an equation where the dependent variable is exports.

The effect size is obtained from an equation where the dependent variable is imports (Reference category).

The infrastructure variable measures roads or railroads.

The infrastructure variable measures port or airport infrastructure.

The infrastructure variable measures communication infrastructure.

The infrastructure measure is a composite index made from multiple types of infrastructure (Reference category).

All economies in which the infrastructure is measured are developed.

All economies in which the infrastructure is measured are developing.

The study focuses on samples that include both developing and developed economies (Reference category).

The units of observation are sub-national regions or firms.

The primary study uses more than one time period.

The dependent variable is scaled by GDP, or a common single indicator such as a product or a sum of the exporter and importer GDP is included as an explanatory variable. 
Table 3.3. Variable definitions (CONT'D)

Variable label

Estimation excludes other infrastructure type(s)

Model does not control of transit or partner infrastructur

Equation excludes multilateral resistances

Equation excludes income

Tariffs or trade agreements not considered

Equation excludes spatial/geographic variables

Equation excludes education and human capital

Population not considered

Governance variable(s) not included

Equation excludes exchange rate

Equation excludes colonial, cultural, linguistic relations

\section{Other study characteristics}

Highly ranked journals

Advocacy
Definition

The equation takes into account only one kind of infrastructure, or the measured infrastructure type is not a composite index made from multiple types.

The model considers the infrastructure of only one trade partner, without taking into account the infrastructure of the other partner or the transit infrastructure.

Study does not specifically control for multilateral resistance terms or use importer and exporter fixed effects.

GDP, per capita GDP, or per capita income difference is not included as a separate variable.

Estimation does not control for the effects of tariffs or trade agreements/blocks.

Landlockedness, distance, or adjacency is not included.

An education or human capital variable is not included.

Population is not included as a separate variable.

A variable controlling for government effectiveness, corruption, rule of law, accountability, business regulation, or regulatory quality is not included.

An exchange rate variable is not included.

Colonial or cultural relationships are not accounted for.

Equals one if the study is published in a journal with rank $\mathrm{A}^{*}, \mathrm{~A}$, or $\mathrm{B}$, equals zero if the rank is $\mathrm{C}$ or $\mathrm{D}$, using ABDC (2010) ranking.

Publisher of the Study is World Bank, OECD, WTO, or UN. 
TABLE 3.4

EFFECT SIZES BY DIRECTION OF TRADE

\begin{tabular}{|c|c|c|c|c|c|c|c|c|}
\hline & \multicolumn{4}{|c|}{ Exporter Infrastructure } & \multicolumn{4}{|c|}{ Importer Infrastructure } \\
\hline & Obs & Mean & Min & $\operatorname{Max}$ & Obs & Mean & Min & $\operatorname{Max}$ \\
\hline Exports & 129 & 0.50 & -1.19 & 1.88 & 70 & 0.22 & -1.40 & 1.78 \\
\hline Imports & 108 & 0.15 & -0.39 & 0.61 & 72 & 0.09 & -0.44 & 0.59 \\
\hline Overall & 237 & 0.34 & -1.19 & 1.88 & 142 & 0.16 & -1.40 & 1.78 \\
\hline
\end{tabular}

TABLE 3.5

EFFECT SIZES BY METHODOLOGY

\begin{tabular}{rrrrrrrrr}
\hline \hline & \multicolumn{4}{c}{ Exporter Infrastructure } & \multicolumn{4}{c}{ Importer Infrastructure } \\
& Obs & Mean & Min & Max & Obs & Mean & Min & Max \\
\hline Heckman Sample Selection, Tobit, or Probit & 82 & 0.33 & -1.19 & 1.76 & 15 & 0.49 & -0.69 & 1.68 \\
IV or Other Control for Endogeneity & 24 & 0.44 & 0.01 & 1.88 & 19 & 0.15 & -0.23 & 0.29 \\
Other Estimation Method & 133 & 0.32 & -0.66 & 1.69 & 108 & 0.11 & -1.40 & 1.78 \\
Overall & $237^{a}$ & 0.34 & -1.19 & 1.88 & 142 & 0.16 & -1.40 & 1.78 \\
\hline
\end{tabular}

${ }^{a}$ As stated earlier, Elbadawi et al. (2006) uses IV and Tobit, resulting the observations to sum to 239 rather than 237 .

TABLE 3.6

EFFECT SIZES BY INFRASTRUCTURE CATEGORY

\begin{tabular}{rrrrrrrrrr}
\hline \hline & \multicolumn{4}{c}{ Exporter Infrastructure } & \multicolumn{3}{c}{ Importer Infrastructure } \\
& Obs & Mean & \multicolumn{1}{c}{ Min } & Max & Obs & Mean & Min & Max \\
\hline Land Transport Infrastructure & 43 & 0.36 & -0.66 & 1.61 & 22 & 0.15 & -1.4 & 1.78 \\
Maritime or Air Transport Infrastructure & 13 & 0.16 & -0.07 & 0.61 & 11 & 0.14 & -0.1 & 0.59 \\
Communication Infrastructure & 56 & 0.08 & -1.19 & 0.71 & 20 & 0.12 & -0.21 & 0.58 \\
Composite Measure (Index) & 125 & 0.47 & -0.9 & 1.88 & 89 & 0.17 & -0.69 & 1.68 \\
Overall & 237 & 0.34 & -1.19 & 1.88 & 142 & 0.16 & -1.40 & 1.78 \\
\hline
\end{tabular}

TABLE 3.7

EFFECT SIZES BY THE DEVELOPMENT LEVEL OF THE ECONOMY IN WHICH THE INFRASTRUCTURE IS LOCATED

\begin{tabular}{rrrrr|rrrr}
\hline \hline & \multicolumn{4}{c}{ Exporter Infrastructure } & \multicolumn{3}{c}{ Importer Infrastructure } \\
& Obs & Mean & Min & Max & Obs & Mean & Min & Max \\
\hline Developed Economy & 9 & 0.32 & 0.12 & 0.52 & 11 & 0.05 & -0.23 & 0.34 \\
Developing Economy & 72 & 0.49 & -1.19 & 1.88 & 11 & 0.07 & -1.40 & 1.78 \\
Both Types of Economies (Mixed Sample) & 156 & 0.27 & -0.90 & 1.44 & 120 & 0.18 & -0.69 & 1.68 \\
Overall & 237 & 0.34 & -1.19 & 1.88 & 142 & 0.16 & -1.40 & 1.78 \\
\hline
\end{tabular}


TABLE 3.8

EFFECT SIZES BY PUBLICATION QUALITY

\begin{tabular}{rrrrr|rrrr}
\hline \hline & \multicolumn{4}{c}{ Exporter Infrastructure } & \multicolumn{3}{c}{ Importer Infrastructure } \\
& Obs & Mean & Min & Max & Obs & Mean & Min & Max \\
\hline Highly Ranked Journals & 67 & 0.40 & -0.90 & 1.88 & 44 & 0.20 & -0.23 & 1.68 \\
Other Journals and Unpublished & 170 & 0.31 & -1.19 & 1.69 & 98 & 0.14 & -1.40 & 1.78 \\
Overall & 237 & 0.34 & -1.19 & 1.88 & 142 & 0.16 & -1.40 & 1.78 \\
\hline
\end{tabular}

The raw mean values that are presented in Tables 3.4 to 3.8 must be treated with caution, as they pool the information obtained from primary studies without considering the standard errors of the estimates. If there is no unobserved heterogeneity in the meta-data, and study characteristics do not play a role in explaining the variation in the estimated effect sizes, the fixed effect (FE) combined estimate is a more efficient average than the ordinary mean (Genc et al., 2012). The FE estimate is a weighted average of effect sizes where the inverse of the estimated variance of each effects size is taken as the weight (Genc et al., 2012). If there is heterogeneity among studies, but not in a systematic way that can be measured by study characteristics, the Random Effect (RE) weighted average accounts for such variability. We calculated the FE and RE estimates as described by Poot (2014) and others.

Because effect sizes come from studies with different geographical coverage, methodology, and model specifications, it is questionable whether there would be an underlying universal effect size. This can be formally confirmed by means of a homogeneity test using a commonly used " $Q$-statistic" (Engels et al., 2000). The $Q$-statistic (computation as in Peters et al. 2010) tests if the primary studies share a common effect size and whether an FE estimate is relevant to the analysis (Poot, 2014). After combining $K$ effect sizes, if the resulting $Q$-statistic from this homogeneity test is greater than the upper-tail critical value of the chi-square distribution with $K-1$ degrees of freedom, then the variance in effect sizes obtained from the primary studies is significantly greater than what can be observed due to random variation around a common effect size (Shadish and Haddock, 1994). If the existence of a shared 
true effect is rejected, the FE approach is not suitable and only the RE estimates should be considered (Poot, 2014).

The $Q$-statistics for exporter infrastructure and importer infrastructure respectively are about 33174.7 and about 4596.1 which both exceed the critical value of 493.6. Based on this outcome of the $Q$-test, we conclude that effect sizes are from a highly heterogeneous pool of studies, and FE weighted average effect sizes are not meaningful. ${ }^{9}$ The RE average effect sizes for exporter and importer infrastructure are 0.167 and 0.145 respectively. Consequently, the result that exporter infrastructure is more influential on trade than importer infrastructure is supported. The $\mathrm{RE}$ estimates suggest that an enhancement in exporter infrastructure of 1 percent would increase annual merchandise trade by about 0.17 percent while importer infrastructure increases trade by about 0.15 percent. In the next section we re-assess this conclusion by controlling for study characteristics and publication bias.

\subsection{Meta-regression Models}

The statistical consequence of the potential unwillingness by researchers or reviewers to publish statistically insignificant results is defined as "publication bias" or "file drawer bias." The actions leading to publication bias can be the efforts of the researchers using small samples towards obtaining large-magnitude estimates (that are statistically significant) while researchers using large samples do not need to exhibit such efforts and report smaller estimates that are still statistically significant. This selection process results in positive correlation between the reported effect size and its standard error (Stanley, 2005; Stanley et al., 2008). As an initial exploration of the possibility of such bias we apply Egger's regression test ${ }^{10}$ (Egger et al., 1997) and the Fixed Effects Extended

\footnotetext{
${ }^{9}$ The FE estimate for exporter infrastructure is -0.002. For importer infrastructure it is 0.044 .

${ }^{10}$ Egger's regression model can be represented as $\hat{\beta}_{i}=\alpha+\rho S e_{i}+\epsilon_{i}$ with the variance of $\epsilon_{i}$ proportional to $1 / S e_{i}^{2}$ where $\hat{\beta}_{i}$ and $S e_{i}$ are the observed effect size and the
} 
Egger Test ${ }^{11}$ (Peters et al., 2010). The results of both tests for exporter and importer infrastructure are reported in Table 3.9. Both variants of the test yield significant coefficients on the bias term when testing for publication bias in the estimates of the impact of exporter infrastructure. The evidence for bias in the estimation of the impact of importer infrastructure is less conclusive, having been confirmed with the Egger test but not with the extended Egger test. The greater bias in estimating the exporter infrastructure impact will also be demonstrated with the Hedges et al. (1992) model of publication bias to which we now turn.

TABLE 3.9

EgGer TESTS

\begin{tabular}{lcc|cc}
\hline \hline & \multicolumn{2}{c}{ Egger Test } & \multicolumn{2}{c}{ Extended Egger Test } \\
& Exporter Inf. & Importer Inf. & Exporter Inf. & Importer Inf. \\
\hline \multirow{2}{*}{ Bias } & & & & \\
& $7.009^{* * *}$ & $2.308^{* * *}$ & $4.318^{* * *}$ & -0.464 \\
Observations & $(0.632)$ & $(0.566)$ & $(0.736)$ & $(0.442)$ \\
R-squared & 237 & 142 & 237 & 142 \\
\hline
\end{tabular}

Standard errors in parentheses

${ }^{* * *} p<0.01,{ }^{* *} p<0.05,{ }^{*} p<0.1$

The Hedges model is an extension of the RE model in which it is assumed that the likelihood of a result being publicly reported is greatest when the associated $p$-value of the coefficient of the variable of interest is smaller than 0.01 . While this likelihood remains unknown, two relative probabilities, denoted here by $\omega_{2}$ and $\omega_{3}$, are associated with the cases:

associated standard error obtained from study $i$ respectively, $\alpha$ is the intercept and $\epsilon_{i}$ is the error term. The bias is measured by $\rho$. If $\rho$ is significantly different from zero, this is a sign of publication bias (Peters et al., 2010)

${ }^{11}$ The FE Extended Egger's Test extends the base model presented in the previous footnote by including a group of covariates: $\hat{\beta}_{i}=\alpha+\rho S e_{i}+$ group $_{i}+\epsilon_{i}$ (Peters et al., 2010). The covariates within "group" are the same list of variables that are used later for the MRA analyses in this study. 
$0.01<p<0.05$ and $p>0.05$ respectively. We use the method proposed by Ashenfelter et al. (1999) to formulate a likelihood function to estimate $\omega_{2}$ and $\omega_{3}$. These parameters should be equal to 1 if publication bias is not present. Table 3.10 presents the estimates associated with the Hedges publication bias procedure. In part (a) of Table 3.10 we consider the case in which there is no observed heterogeneity assumed, i.e. there are no study characteristics that act as covariates. In part (b) of Table 3.10, covariates have been included. The model is estimated under the restriction that the probabilities of publication are all the same on the RHS of the table, while the LHS of the table estimates the relative probabilities with maximum likelihood.

On the LHS of Table 3.10 (a) we see that less significant estimates are less likely to be reported. The corresponding weights for $0.01<p<0.05$ and $p>0.05$ are 0.739 and 0.137 for exporter's infrastructure, and 0.280 and 0.120 for imports. The RHS shows the results of the restricted model which assumes $\omega_{2}=\omega_{3}=1$ (no publication bias). The chisquare critical value at 1 percent level with two degrees of freedom is 9.21. Two times the difference between the log-likelihoods of assuming and not assuming publication bias is 63.28 for exporter's infrastructure without study characteristics and 51.2, with study characteristics - in both cases greatly exceeding the critical value and providing evidence for publication bias at the 1 percent level. Similarly, evidence for the existence of publication bias is also observed for importer infrastructure, with test statistics of 53.62 and 151.8 for without and with covariates respectively.

We can also see that residual heterogeneity decreases considerably upon the introduction of study characteristics for both exporter and importer infrastructure (from 0.341 to 0.255 and from 0.231 to 0.0302 respectively). Accounting for publication bias and study heterogeneity (Table 3.10b) lowers the RE estimate of the exporter infrastructure elasticity from 0.300 to 0.254 but leaves the RE estimate of the importer infrastructure elasticity relatively unaffected ( 0.256 and 0.259 respectively). This is consistent with the result of the extended Egger test reported above.

Taking into account the heterogeneity that is apparent in our data set, 
as demonstrated formally by the Q-statistic, we now conduct MRA in order to account for the impact of study characteristics on study effect sizes.

The simplest MRA assumes that there are $S$ independent studies $(s=$ $1,2, \ldots, S)$ which each postulate the classic regression model $\boldsymbol{y}(s)=$ $\boldsymbol{X}(s) \boldsymbol{\beta}(s)+\boldsymbol{\epsilon}(s)$, with the elements of $\boldsymbol{\epsilon}(s)$ identically and independently distributed with mean 0 and variance $\sigma^{2}(s)$. Study $s$ has $N(s)$ observations and the vector $\boldsymbol{\beta}(s)$ has dimension $K(s) \times 1$. The first element of this vector is the parameter of interest and has exactly the same interpretation across all studies (in our case it is either the exporter infrastructure elasticity of trade or the importer infrastructure elasticity of trade).

Under these assumptions, a primary study would estimate $\boldsymbol{\beta}(s)$ by the OLS estimator $\hat{\boldsymbol{\beta}}(s)=\left[\boldsymbol{X}(s)^{\prime} \boldsymbol{X}(s)\right]^{-1}\left[\boldsymbol{X}(s)^{\prime} \boldsymbol{y}(s)\right]$, which is best asymptotically normal distributed with mean $\boldsymbol{\beta}(s)$ and covariance matrix $\sigma^{2}(s)\left[\boldsymbol{X}(s)^{\prime} \boldsymbol{X}(s)\right]^{-1}$. The $S$ estimates of the parameter of interest are the effect sizes. We observe the effect sizes $\hat{\beta}_{1}(1), \hat{\beta}_{1}(2), \ldots, \hat{\beta}_{1}(s)$. Given the data generating process for the primary studies,

$$
\hat{\beta}_{1}(s)=\beta_{1}(s)+\left[\left[\boldsymbol{X}(s)^{\prime} \boldsymbol{X}(s)\right]^{-1} \boldsymbol{X}(s)^{\prime} \boldsymbol{\epsilon}(s)\right]_{1}
$$

which are consistent and efficient estimates of the unknown parameters $\beta_{1}(1), \beta_{1}(2), \ldots, \beta_{1}(S)$. These effect sizes have estimated variances $v(1), v(2), \ldots, v(S)$. In study $s, v(s)$ is the top left element of the matrix $\hat{\sigma}^{2}(s)\left[\boldsymbol{X}(s)^{\prime} \boldsymbol{X}(s)\right]^{-1}$ with $\hat{\sigma}^{2}(s)=\left[\boldsymbol{e}(s)^{\prime} \boldsymbol{e}(s)\right]^{\prime} / N(s)$, and $\boldsymbol{e}(s)=\boldsymbol{y}(s)-\boldsymbol{X}(s) \hat{\boldsymbol{\beta}}(s)$ is the vector of least square residuals.

MRA assumes that there are $P$ known moderator (or predictor) variables $M_{1}, M_{2}, \ldots, M_{P}$ that are related to the unknown parameters of interest $\beta_{1}(1), \beta_{1}(2), \ldots, \beta_{1}(S)$ via a linear model as follows:

$$
\beta_{1}(s)=\gamma_{0}+\gamma_{1} M_{s 1}+\ldots+\gamma_{P} M_{s P}+\eta_{s}
$$


in which $M_{s j}$ is the value of the $j$ th moderator variable associated with effect size $s$ and the $\eta_{s}$ are independently and identically distributed random variables with mean 0 and variance $\tau^{2}$ (the between-studies variance). Thus, equation (3.8) allows for both observable heterogeneity (in terms of observable moderator variables) and unobservable heterogeneity (represented by $\eta_{s}$ ). By combining (3.7) and (3.8), the MRA model becomes

$$
\hat{\beta}_{1}(s)=\gamma_{0}+\gamma_{1} M_{s 1}+\ldots \gamma_{P} M_{s P}+\{\underbrace{\eta_{s}+\left[\left[\boldsymbol{X}(s)^{\prime} \boldsymbol{X}(s)\right]^{-1} \boldsymbol{X}(s)^{\prime} \epsilon(s)\right]_{1}}_{\text {Error term of MRA }}\}
$$

with the term in the curly brackets being the error term of the MRA. The objective of MRA is to find estimates of $\gamma_{0}, \gamma_{1}, \ldots \gamma_{P}$ that provide information on how observed estimates of the coefficients of the focus variable are linked to observed study characteristics. Typically, the meta-analyst observes for each $s=1,2, \ldots, S: \hat{\beta}_{1}(s)$; its estimated variance $\hat{\sigma}^{2}(s)\left[\left[\boldsymbol{X}(s)^{\prime} \boldsymbol{X}(s)\right]^{-1}\right]_{11}$; the number of primary study observations $N(s)$, and information about the variables that make up $\boldsymbol{X}(s)$, possibly including means and variances, but not the actual data or the covariances between regressors. ${ }^{12}$ The $P$ known moderator variables $M_{1}, M 2, \ldots M_{P}$ are assumed to capture information about the covariates and the estimation method in case the estimations were obtained by techniques other than OLS. Clearly, the error term in regression model (3.9) is heteroskedastic and generates a between-study variance due to $\eta_{s}$ and a within-study variance due to $\left[\left[\boldsymbol{X}(s)^{\prime} \boldsymbol{X}(s)\right]^{-1} \boldsymbol{X}(s)^{\prime} \boldsymbol{\epsilon}(s)\right]_{1}$.

\footnotetext{
${ }^{12}$ If covariances are known, Becker and $\mathrm{Wu}$ (2007) suggest an MRA that pools estimates of all regression parameters, not just of the focus variable, and that can be estimated with feasible GLS.
} 
TABLE 3.10

Hedges Publication bias

(a) Study characteristics not considered

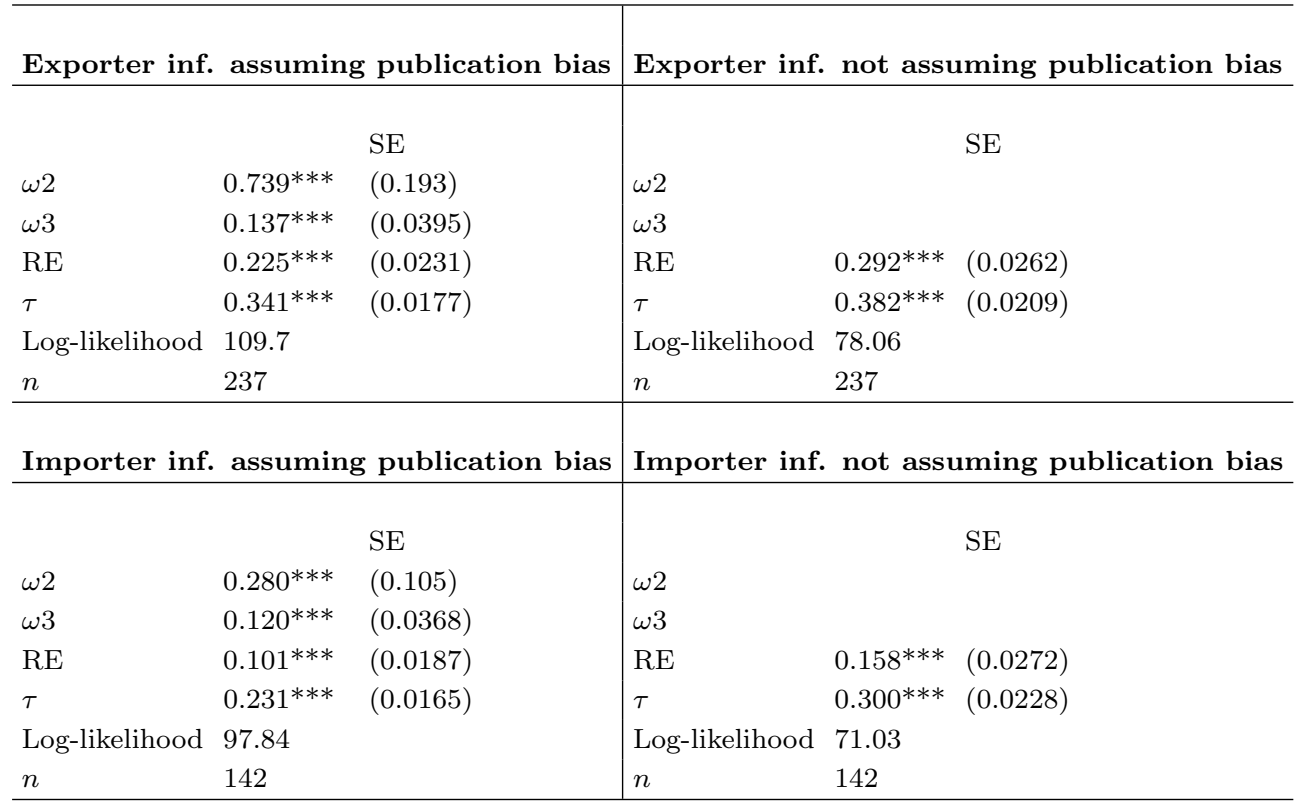

(b) Study characteristics considered

Exporter inf. assuming publication bias Exporter inf. not assuming publication bias

\begin{tabular}{|c|c|c|c|c|c|}
\hline & & $\mathrm{SE}$ & & & $\mathrm{SE}$ \\
\hline$\omega 2$ & $0.747 * * *$ & $(0.196)$ & $\omega 2$ & & \\
\hline$\omega 3$ & $0.156^{* * *}$ & $(0.0464)$ & $\omega 3$ & & \\
\hline $\mathrm{RE}$ & $0.254^{* * *}$ & $(0.0199)$ & $\mathrm{RE}$ & $0.300^{* * *}$ & $(0.021)$ \\
\hline$\tau$ & $0.255^{* * *}$ & $(0.0145)$ & $\tau$ & $0.273^{* * *}$ & $(0.0163)$ \\
\hline Log-likelihood & 168.3 & & Log-likelihood & 142.7 & \\
\hline$n$ & 237 & & $n$ & 237 & \\
\hline \multirow[t]{2}{*}{ Importer inf. } & assuming & publication bias & \multicolumn{3}{|c|}{ Importer inf. not assuming publication bias } \\
\hline & & $\mathrm{SE}$ & & & $\mathrm{SE}$ \\
\hline$\omega 2$ & $0.0716^{* * *}$ & $(0.0266)$ & $\omega 2$ & & \\
\hline$\omega 3$ & $0.0142 * * *$ & $(0.00409)$ & $\omega 3$ & & \\
\hline $\mathrm{RE}$ & $0.259 * * *$ & $(0.0191)$ & $\mathrm{RE}$ & $0.256^{* * *}$ & $(0.0499)$ \\
\hline$\tau$ & $0.0302^{* * *}$ & $(0.0059)$ & $\tau$ & $0.136^{* * *}$ & $(0.016)$ \\
\hline Log-likelihood & 210 & & Log-likelihood & 134.1 & \\
\hline$n$ & 142 & & $n$ & 142 & \\
\hline
\end{tabular}


We apply two different estimation methods for equation $(3.9):^{13}$

a. Restricted Maximum Likelihood (REML): In REML the betweenstudy variance is estimated by maximizing the residual (or restricted) log likelihood function and a WLS regression weighted by the sum of the between-study and within-study variances is conducted to obtain the estimated coefficients (Harbord and Higgins, 2008). The standard error does not enter as an individual variable into this specification.

b. The publication bias corrected maximum likelihood procedure proposed by Hedges et al. (1992) and outlined above.

The results of the estimation of equation (3.9) with the REML and Hedges estimators are shown in Table 3.11. All explanatory variables are transformed in deviations from their original means. We analyze the results separately for each category of variables.

\subsubsection{Methodology}

Results from the Hedges model suggest that studies which take zero trade flows into account by using Heckman sample selection, Tobit, or Probit models, on average, estimate a lower effect size for exporter infrastructure, and a higher effect size for importer infrastructure. For robustness checks, OLS and WLS estimates are reported in the appendix. On the matter of sample selections, the results are not consistent across MRAs. In what follows, we will pay most attention to the results of the Hedges model since this is the only model that accounts for publication bias but emphasize those results that are found in the other MRAs as well.

\footnotetext{
${ }^{13}$ For robustness checks we also ran OLS and WLS regressions with standard errors clustered by primary study (with weights being the number of observations from each primary regression equation) and variables transformed to deviations from means, so that the estimated constant term becomes the estimated mean effect size. The results are reported in the appendix.
} 
According to both the REML and Hedges results, studies that use instrumental variable methods to deal with potential endogeneity observe a larger impact of exporter infrastructure on trade. Consequently, econometric methodology is an important study characteristic that affects the results. Not accounting for endogeneity of exporter infrastructure leads to an underestimation of its impact on trade. This is not the case for importer (consumer) infrastructure.

Whether a primary study uses a gravity model or not does not seem to have an influence. For importer infrastructure this variable drops out. This is because, naturally, there are no effect sizes in our sample resulting from a regression where the importing partner's infrastructure is included and the model is not in gravity form. Implicitly, the inclusion of the Gravity model dummy also asks the question if the distance between trade partners has been considered in the primary estimations, as distance is an essential component of a gravity specification.

\subsubsection{The point at which the trade is measured}

In both the REML and Hedges estimations, the coefficient of the dummy Dependent variable is exports is significant and positive for exporter infrastructure, suggesting that own infrastructure has a greater impact when trade is measured by export data rather than by import data. This is also found in the OLS and WLS MRAs in the appendix. As discussed in Section 3, in a primary study where all bilateral trading partners would be included and all trade is measured with transaction costs included (cif), the two effect sizes must be equal. However, data on any trade flow may differ depending on measurement at the point of shipment or at the point of importation. Moreover, as noted previously, trade matrices may not be square, such as in an analysis of developing country exports to developed countries. For the same variable, the Hedges model yields a significant and negative coefficient for importer infrastructure, suggesting that the impact of the infrastructure located in the importing economy is lower when measured with respect to the exports of its partner than with respect to its own imports. 
Using the Hedges model, we can predict the overall impacts of exporter (producer) infrastructure and importer (consumer) infrastructure by combining these coefficients with the constant terms, which measure the overall average effects. The results can be directly compared with the "raw" averages reported in Table 3.4. We get:

- The own infrastructure of country $i$ has an average effect size of $0.254+0.345=0.599$ on the exports of $i$;

- The own infrastructure of country $i$ has an average effect size of 0.259 on the imports of $i$;

- The infrastructure in the partner country $j$ of the exporting country $i$ has an average effect size of 0.254 on the imports of $i$;

- The infrastructure in the partner country $j$ of the exporting country $i$ has an average effect size of $0.259-0.126=0.133$ on the exports of $i$.

We see that after controlling for heterogeneity and publication bias, the exporter infrastructure effect continues to be larger when measured with export data than with import data, (0.599 versus 0.254 above, compared with 0.50 and 0.15 respectively in Table 3.4 ), while for importer infrastructure the opposite is the case ( 0.133 versus 0.259 above, and 0.22 versus 0.09 respectively in Table 3.4 ). The most important result from this analysis is that from the perspective of any given country, the impact of own infrastructure on net trade (assuming roughly balanced gross trade) is $0.599-0.259=0.340$. Alternatively, if we take the average of the exporter infrastructure elasticities 0.599 and 0.254 , and subtract the average of the importer infrastructure elasticities (0.133 and 0.259), we get a net trade effect of 0.23 . Averaging the calculations from both perspectives, an increase in own infrastructure by 1 percent increases net trade by about 0.3 percent. We address the macroeconomic implication of this finding in Section 3.8. 


\subsubsection{Infrastructure category}

As discussed earlier, infrastructure is defined as a collection, or portfolio of various components. Consequently, in our estimations, four common measurements of infrastructure are accounted for (land, maritime or air, communication, and a composite index). Aside from the REML model for importer infrastructure, all our estimations suggest that land transport infrastructure is estimated to have a larger effect size on trade than the other infrastructure categories on average. The Hedges model suggests that maritime and air transportation infrastructure and communication infrastructure on the importer side have higher average effect sizes compared to elasticities obtained from composite infrastructure indexes.

\subsubsection{Development level of the economy in which the in- frastructure is located}

Both the REML and Hedges results suggest that exporter infrastructure matters more for trade if the exporting economy is developing rather than developed (also shown by the OLS model in the appendix). This result was already noted previously and is commonly found in the literature. Moreover, importer infrastructure is less influential in trade when the importing economy is developed (also shown with the WLS model in the appendix).

\subsubsection{Sample structure}

The Hedges, REML, OLS and WLS MRAs all suggest that a lower infrastructure elasticity of trade for importer infrastructure has been observed in estimates obtained from studies where the units of analysis were sub-regional or firm level. The same is found for exporter infrastructure, but only in the Hedges model. Sub-regional samples force the location where trade takes place and the location of infrastructure to be measured (spatially) closer to one another. Therefore, such samples do 
not capture spillovers to the rest of the economy. The negative result on the variable Sub-national or firm level suggests that the estimated macro effects are larger than the micro effects.

\subsubsection{Model specification}

The dummy variables are defined such that they are equal to unity when a particular covariate has been omitted from the primary regression. Consequently, the coefficients provide an explicit measure of omitted variable bias. The Hedges model results show some evidence that for estimations that do not control for other infrastructure types (for example, if only road infrastructure is considered), the impact of importer infrastructure on trade is likely to be overestimated. The REML and Hedges models suggest that similar positive omitted variable bias arises for the importer infrastructure elasticity of trade when exporter infrastructure is not jointly considered (this is also found in the OLS and WLS MRAs).

Both models also suggest that excluding income and tariff or trade agreement variables can bias the estimate on exporter infrastructure downwards, while - based on the Hedges results - an upward bias for importer infrastructure can result if tariffs or trade agreements are not taken into account. Both models suggest that omitting variables for education or human capital can cause a downward bias in the estimation of the importer infrastructure elasticity of trade (also found in the OLS and WLS MRAs). The same can be said for the estimation of both the exporter and importer infrastructure effect size based on the results of both models if governance-related variables such as rule of law and corruption are omitted. Not considering population can cause the effect size of importer elasticity to be overestimated according to the Hedges results. Omitting the exchange rate in the trade regression leads to upward bias in the estimate for exporter infrastructure (also confirmed by the OLS and WLS MRAs). 


\subsubsection{Nature of publication}

The Hedges model provides some evidence that studies, which were published in highly ranked journals, have estimated a larger effect size of importer infrastructure compared to other studies. A similar result is visible for the advocacy variable: research published by institutes with potential advocacy motives for announcing a larger infrastructure effect have estimated, on average, a higher effect size for importer infrastructure. All advocacy coefficients are positive, but for exporter infrastructure, only the result of the WLS estimation reported in the appendix is statistically significant.

\subsubsection{Model prediction}

A final useful exercise is to consider the goodness of fit of an MRA with respect to the set of effect sizes reported in the original studies. For this purpose, we predicted the mean squared error (MSE) of the comparison between the observed effect sizes and those predicted by the REML model for each study (predictions by the Hedges model are more cumbersome). The MSE for each study is reported in Table 3.12a for exporter infrastructure and Table $3.12 \mathrm{~b}$ for importer infrastructure. Among the studies that contributed to both MRAs, the REML soundly describes the studies of Raballand (2003), Grigoriou (2007), Bandyopadhyay (1999), Carrère (2006) and Brun et al. (2005). On the other hand, the studies of Iwanow and Kirkpatrick (2009), Fujimura and Edmonds (2006) and Marquez-Ramos and Martinez-Zarzoso (2005) yield results that are not closely aligned with what the REML MRAs suggested. 
TABle 3.11

Estimation RESULTS

\begin{tabular}{|c|c|c|c|c|}
\hline & \multicolumn{2}{|c|}{ REML } & \multicolumn{2}{|c|}{ Hedges } \\
\hline & $\begin{array}{c}\text { Exporter } \\
\text { Infrastructure }\end{array}$ & $\begin{array}{c}\text { Importer } \\
\text { Infrastructure }\end{array}$ & $\begin{array}{c}\text { Exporter } \\
\text { Infrastructure }\end{array}$ & $\begin{array}{c}\text { Importer } \\
\text { Infrastructure }\end{array}$ \\
\hline Methodology & & & & \\
\hline $\begin{array}{l}\text { Model accounts for zero trade selec- } \\
\text { tion (Heckman, Tobit, Probit) }\end{array}$ & $\begin{array}{c}-0.103 \\
(0.0803)\end{array}$ & $\begin{array}{l}-0.128 \\
(0.143)\end{array}$ & $\begin{array}{l}-0.108^{*} \\
(0.0629)\end{array}$ & $\begin{array}{l}0.0888^{* *} \\
(0.0371)\end{array}$ \\
\hline $\begin{array}{l}\text { Model accounts for endogeneity } \\
\text { (IV-based estimation) }\end{array}$ & $\begin{array}{l}0.256^{* *} \\
(0.113)\end{array}$ & $\begin{array}{l}-0.0453 \\
(0.111)\end{array}$ & $\begin{array}{l}0.245^{* * *} \\
(0.0949)\end{array}$ & $\begin{array}{l}-0.0187 \\
(0.0194)\end{array}$ \\
\hline Gravity model & $\begin{array}{l}-0.362 \\
(0.346)\end{array}$ & & $\begin{array}{l}-0.347 \\
(0.296)\end{array}$ & \\
\hline The point at which trade is mea & sured & & & \\
\hline Dependent variable is exports & $\begin{array}{c}0.410^{* * *} \\
(0.143)\end{array}$ & $\begin{array}{l}-0.117 \\
(0.138)\end{array}$ & $\begin{array}{c}0.345^{* * *} \\
(0.115)\end{array}$ & $\begin{array}{c}-0.126^{* * *} \\
(0.0366)\end{array}$ \\
\hline Infrastructure category & & & & \\
\hline Land transport infrastructure & $\begin{array}{l}0.197^{* *} \\
(0.0770)\end{array}$ & $\begin{array}{c}0.106 \\
(0.0889)\end{array}$ & $\begin{array}{l}0.170^{* * *} \\
(0.0611)\end{array}$ & $\begin{array}{c}0.0743^{* * *} \\
(0.0245)\end{array}$ \\
\hline Maritime or air infrastructure & $\begin{array}{c}0.0239 \\
(0.0877)\end{array}$ & $\begin{array}{c}0.115 \\
(0.117)\end{array}$ & $\begin{array}{c}0.0413 \\
(0.0691)\end{array}$ & $\begin{array}{l}0.592^{* *} \\
(0.0254)\end{array}$ \\
\hline Communication infrastructure & $\begin{array}{c}0.0611 \\
(0.0901)\end{array}$ & $\begin{array}{c}0.0591 \\
(0.0835)\end{array}$ & $\begin{array}{c}0.0674 \\
(0.0727)\end{array}$ & $\begin{array}{c}0.0555^{* *} \\
(0.0229)\end{array}$ \\
\hline Composite measure (index) & Reference dummy & & & \\
\hline $\begin{array}{l}\text { Development level of the econ } \\
\text { which infrastructure is located }\end{array}$ & my in & & & \\
\hline Developing economy & $\begin{array}{c}0.229^{* * *} \\
(0.0705)\end{array}$ & $\begin{array}{l}-0.138 \\
(0.141)\end{array}$ & $\begin{array}{c}0.169^{* * *} \\
(0.0574)\end{array}$ & $\begin{array}{r}-0.00963 \\
(0.0383)\end{array}$ \\
\hline Developed economy & $\begin{array}{c}0.163 \\
(0.203)\end{array}$ & $\begin{array}{r}-0.0547 \\
(0.132)\end{array}$ & $\begin{array}{c}0.122 \\
(0.159)\end{array}$ & $\begin{array}{c}-0.124^{* * *} \\
(0.0320)\end{array}$ \\
\hline $\begin{array}{l}\text { Both types of economies } \\
\text { (mixed sample) }\end{array}$ & Reference dummy & & & \\
\hline
\end{tabular}


TABLE 3.11

ESTIMATION RESULTS (CONT'D)

\begin{tabular}{|c|c|c|c|c|}
\hline & \multicolumn{2}{|c|}{ REML } & \multicolumn{2}{|c|}{ Hedges } \\
\hline & $\begin{array}{c}\text { Exporter } \\
\text { Infrastructure }\end{array}$ & $\begin{array}{c}\text { Importer } \\
\text { Infrastructure }\end{array}$ & $\begin{array}{c}\text { Exporter } \\
\text { Infrastructure }\end{array}$ & $\begin{array}{c}\text { Importer } \\
\text { Infrastructure }\end{array}$ \\
\hline Sample structure & & & & \\
\hline Sub-national or firm level & $\begin{array}{l}-0.383 \\
(0.269)\end{array}$ & $\begin{array}{c}-0.474^{* *} \\
(0.203)\end{array}$ & $\begin{array}{c}-0.476^{* *} \\
(0.204)\end{array}$ & $\begin{array}{c}-0.495^{* * *} \\
(0.0550)\end{array}$ \\
\hline No cross-section & $\begin{array}{l}0.0661 \\
(0.111)\end{array}$ & $\begin{array}{l}0.190^{*} \\
(0.106)\end{array}$ & $\begin{array}{c}0.0951 \\
(0.0919)\end{array}$ & $\begin{array}{l}0.161^{* * *} \\
(0.0342)\end{array}$ \\
\hline Model specification & & & & \\
\hline Constrained model & $\begin{array}{l}0.0469 \\
(0.180)\end{array}$ & $\begin{array}{c}0.314 \\
(0.281)\end{array}$ & $\begin{array}{c}-0.00682 \\
(0.155)\end{array}$ & $\begin{array}{c}0.0758 \\
(0.0623)\end{array}$ \\
\hline $\begin{array}{l}\text { Estimation excludes other } \\
\text { infrastructure categories }\end{array}$ & $\begin{array}{l}0.00950 \\
(0.150)\end{array}$ & $\begin{array}{c}0.255 \\
(0.176)\end{array}$ & $\begin{array}{l}0.0424 \\
(0.126)\end{array}$ & $\begin{array}{l}0.113^{* *} \\
(0.0506)\end{array}$ \\
\hline $\begin{array}{l}\text { Model does not control for transit } \\
\text { or partner infrastructure }\end{array}$ & $\begin{array}{l}-0.188 \\
(0.195)\end{array}$ & $\begin{array}{l}0.644^{* *} \\
(0.296)\end{array}$ & $\begin{array}{l}-0.145 \\
(0.162)\end{array}$ & $\begin{array}{l}0.439^{* * *} \\
(0.0788)\end{array}$ \\
\hline $\begin{array}{l}\text { Equation excludes multilateral } \\
\text { resistances }\end{array}$ & $\begin{array}{l}-0.126 \\
(0.134)\end{array}$ & $\begin{array}{l}0.0399 \\
(0.141)\end{array}$ & $\begin{array}{l}-0.0877 \\
(0.106)\end{array}$ & $\begin{array}{c}0.0474 \\
(0.0360)\end{array}$ \\
\hline Equation excludes income & $\begin{array}{l}-0.535^{*} \\
(0.298)\end{array}$ & & $\begin{array}{l}-0.379^{*} \\
(0.228)\end{array}$ & \\
\hline $\begin{array}{l}\text { Tariffs or trade agreements } \\
\text { not considered }\end{array}$ & $\begin{array}{c}-0.291^{* *} \\
(0.130)\end{array}$ & $\begin{array}{l}0.0943 \\
(0.116)\end{array}$ & $\begin{array}{c}-0.240^{* *} \\
(0.104)\end{array}$ & $\begin{array}{l}0.130^{* * *} \\
(0.0265)\end{array}$ \\
\hline $\begin{array}{l}\text { Equation excludes spa- } \\
\text { tial/geographic variables }\end{array}$ & $\begin{array}{l}-0.0600 \\
(0.116)\end{array}$ & $\begin{array}{c}-0.105 \\
(0.0923)\end{array}$ & $\begin{array}{c}-0.105 \\
(0.0946)\end{array}$ & $\begin{array}{l}0.000848 \\
(0.0161)\end{array}$ \\
\hline $\begin{array}{l}\text { Equation excludes education } \\
\text { and human capital }\end{array}$ & $\begin{array}{l}0.0476 \\
(0.137)\end{array}$ & $\begin{array}{c}-0.911^{* * *} \\
(0.282)\end{array}$ & $\begin{array}{c}0.131 \\
(0.111)\end{array}$ & $\begin{array}{c}-0.829^{* * *} \\
(0.0708)\end{array}$ \\
\hline Population not considered & $\begin{array}{c}0.0466 \\
(0.0821)\end{array}$ & $\begin{array}{c}0.0584 \\
(0.0909)\end{array}$ & $\begin{array}{c}0.0289 \\
(0.0655)\end{array}$ & $\begin{array}{l}0.101^{* * *} \\
(0.0246)\end{array}$ \\
\hline $\begin{array}{l}\text { Governance variable(s) not consid- } \\
\text { ered }\end{array}$ & $\begin{array}{c}-0.395^{* * *} \\
(0.0902)\end{array}$ & $\begin{array}{c}-0.425^{* * *} \\
(0.156)\end{array}$ & $\begin{array}{c}-0.402^{* * *} \\
(0.0731)\end{array}$ & $\begin{array}{c}-0.297^{* * *} \\
(0.0458)\end{array}$ \\
\hline
\end{tabular}


TABLE 3.11

ESTIMATION RESULTS (CONT'D)

\begin{tabular}{|c|c|c|c|c|}
\hline & \multicolumn{2}{|c|}{ REML } & \multicolumn{2}{|c|}{ Hedges } \\
\hline & $\begin{array}{c}\text { Exporter } \\
\text { Infrastructure }\end{array}$ & $\begin{array}{c}\text { Importer } \\
\text { Infrastructure }\end{array}$ & $\begin{array}{c}\text { Exporter } \\
\text { Infrastructure }\end{array}$ & $\begin{array}{c}\text { Importer } \\
\text { Infrastructure }\end{array}$ \\
\hline Equation excludes exchange rate & $\begin{array}{l}0.293^{* * *} \\
(0.0964)\end{array}$ & $\begin{array}{l}0.000271 \\
(0.0852)\end{array}$ & $\begin{array}{l}0.281^{* * *} \\
(0.0779)\end{array}$ & $\begin{array}{l}0.00635 \\
(0.0150)\end{array}$ \\
\hline $\begin{array}{l}\text { Equation excludes colonial, cul- } \\
\text { tural, or linguistic relations }\end{array}$ & $\begin{array}{l}0.0261 \\
(0.179)\end{array}$ & $\begin{array}{c}0.140 \\
(0.126)\end{array}$ & $\begin{array}{l}0.00984 \\
(0.158)\end{array}$ & $\begin{array}{c}0.0296 \\
(0.0463)\end{array}$ \\
\hline Nature of publication & & & & \\
\hline Highly ranked journals & $\begin{array}{l}-0.0261 \\
(0.139)\end{array}$ & $\begin{array}{c}0.316 \\
(0.240)\end{array}$ & $\begin{array}{l}-0.0129 \\
(0.112)\end{array}$ & $\begin{array}{l}0.122^{* *} \\
(0.0560)\end{array}$ \\
\hline Advocacy & $\begin{array}{c}0.128 \\
(0.135)\end{array}$ & $\begin{array}{c}0.362 \\
(0.245)\end{array}$ & $\begin{array}{l}0.0650 \\
(0.112)\end{array}$ & $\begin{array}{l}0.115^{* *} \\
(0.0500)\end{array}$ \\
\hline Constant & $\begin{array}{l}0.302^{* * *} \\
(0.0242)\end{array}$ & $\begin{array}{l}0.258^{* * *} \\
(0.0721)\end{array}$ & $\begin{array}{l}0.254^{* * *} \\
(0.0199)\end{array}$ & $\begin{array}{l}0.259^{* * *} \\
(0.0191)\end{array}$ \\
\hline Log-likelihood & 75.25 & 67.45 & 168.3 & 210.0 \\
\hline $\begin{array}{l}\tau \\
\text { Proportion of between study vari- } \\
\text { ance explained }\end{array}$ & $\begin{array}{l}0.09 \\
0.40\end{array}$ & $\begin{array}{l}0.03 \\
0.66\end{array}$ & & \\
\hline $\begin{array}{l}\text { \% Residual variance due to hetero- } \\
\text { geneity }\end{array}$ & 0.981 & 0.828 & & \\
\hline Observations & 237 & 142 & 237 & 142 \\
\hline
\end{tabular}

Standard errors in parentheses

${ }^{*} p<0.10,{ }^{* *} p<0.05,{ }^{* * *} p<0.01$ 
TABLE $3.12 \mathrm{~A}$

RANKING OF THE STUDIES BY THEIR MEAN SQUARED ERRORS: EXPORTER INFRASTRUCTURE

\begin{tabular}{ll}
\hline \hline Author & MSE \\
\hline Kurmanalieva and Parpiev (2008) & 0.002 \\
Brun et al. (2005) & 0.005 \\
Raballand (2003) & 0.023 \\
Bandyopadhyay (1999) & 0.043 \\
Persson (2007) & 0.053 \\
Carrère (2006) & 0.058 \\
Nordas and Piermartini (2004) & 0.063 \\
Elbadawi (1999) & 0.087 \\
Francois and Manchin (2007) & 0.111 \\
Grigoriou (2007) & 0.151 \\
Njinkeu et al. (2008) & 0.167 \\
Wilson et al. (2004) & 0.202 \\
Martínez-Zarzoso and Nowak-Lehmann (2003) & 0.211 \\
Fujimura and Edmonds (2006) & 0.389 \\
Ninkovic (2009) & 0.442 \\
De (2007) & 0.445 \\
UN Economic Commission for Africa (UNECA) (2013) & 0.518 \\
Vijil and Wagner (2012) & 0.925 \\
Portugal-Perez and Wilson (2012a) & 1.014 \\
Marquez-Ramos and Martinez-Zarzoso (2005) & 1.047 \\
Iwanow and Kirkpatrick (2007) & 1.969 \\
Bouet et al. (2008) & 2.013 \\
Elbadawi et al. (2006) & 7.348 \\
Granato (2008) & 7.727 \\
\hline & \\
&
\end{tabular}


TABLE 3.12B

RANKING OF THE STUDIES BY THEIR MEAN SQUARED ERRORS: IMPORTER INFRASTRUCTURE

\begin{tabular}{ll}
\hline \hline Author & MSE \\
\hline Raballand (2003) & 0.000 \\
Grigoriou (2007) & 0.006 \\
Bandyopadhyay (1999) & 0.012 \\
Carrère (2006) & 0.012 \\
Jansen and Nordås (2004) & 0.014 \\
Brun et al. (2005) & 0.016 \\
Martínez-Zarzoso and Nowak-Lehmann (2003) & 0.020 \\
Wilson et al. (2004) & 0.026 \\
Nordas and Piermartini (2004) & 0.067 \\
Kurmanalieva and Parpiev (2008) & 0.116 \\
Persson (2007) & 0.118 \\
De (2007) & 0.147 \\
Njinkeu et al. (2008) & 0.149 \\
Iwanow and Kirkpatrick (2009) & 0.461 \\
Fujimura and Edmonds (2006) & 0.541 \\
Marquez-Ramos and Martinez-Zarzoso (2005) & 0.541 \\
Lawless (2010) & 0.672 \\
\hline
\end{tabular}




\subsection{Concluding Remarks}

In this study, we have applied meta-analytic techniques to estimate the impact of exporter and importer infrastructure on trade and to examine the factors that influence the estimated elasticities of this impact. The initial data set consisted of 542 estimates obtained from 36 primary studies. We observe evidence that publication (or file drawer) bias exists in the strand of literature in question and apply the Hedges publication bias procedure.

The key result of our research is that the own infrastructure elasticity of the exports of a country is about 0.6 and own infrastructure elasticity on the imports of a country is about 0.3 . This finding suggests that exports would respond to an improvement in the overall trade-related infrastructure more than imports, and that an expansion of the interrelated and integrated components of total trade-related infrastructure may have an attractive return through its impact on the external trade balance.

This result can be further elaborated. Assume that in a given economy, infrastructure is valued at about 50 percent of GDP: ${ }^{14}$ The resource cost of a 1 percent increase in infrastructure would therefore be about 0.5 percent of GDP. As the Hedges MRA results suggest that such an increase in infrastructure will increase exports by about 0.6 percent and imports by about 0.3 percent, starting from a situation of exports and imports being of similar magnitude, net exports will then increase by about 0.3 percent of the value of exports. The impact of this on GDP clearly depends on the openness of the economy (as measured by the exports to GDP ratio) and the short-run and long-run general equilibrium consequences. In turn, these will depend on the assumptions made and the analytical framework adopted. Nevertheless, even under conservative assumptions the additional infrastructure is likely to have an expansionary impact in the short-run (although the size of any multiplier remains debated, see e.g. Owyang et al. 2013), and also in the long-

\footnotetext{
${ }^{14}$ This is a conservative estimate that refers, for example, to the case of Canada. The report by Dobbs et al. (2013) suggests that infrastructure is valued at around 70 percent of GDP.
} 
run through increasing external trade. For reasonable discount rates and sufficiently open economies, it is easy to construct examples that yield attractive benefit-cost ratios for such infrastructure investment. Additionally, it has often been argued that such an expansionary policy may yield further productivity improvements.

The question remains of course what causes this differential impact of infrastructure on exports vis-a-vis imports. Consider the export demand function as presented by Anderson and van Wincoop (2003):

$$
x_{i j}=\left(\frac{\beta_{i} p_{i} t_{i j}}{P_{j}}\right)^{1-\sigma} y_{j}
$$

Equation (3.10) implies that a decline in $t$ due to improved infrastructure raises the demand for country (or region) $i$ 's exports. Given that an exporting firm is a price taker in the foreign market and bears the transportation costs to compete there, increases in the stock or quality of origin infrastructure raise the profitability of exports to all possible destinations. On the other hand, from the point of view of a foreign firm that supplies imports to country $i$, this infrastructure enhancement in the home economy lowers the cost of transportation to one destination only. Thus, an increase in infrastructure affects all exports of the local firm but it only affects a proportion of the exports of the foreign firm. Because imports may be more income elastic than price elastic, the effect of the decrease in the price of imports (which already included the foreign freight and insurance) relative to the domestic price will be small. Consequently, the change in infrastructure in country $i$ impacts the behavior of the foreign firm that produces the imports less than that of the domestic firm that produces exports (assuming the infrastructure in other countries remained constant). Therefore, the marginal impact is at least initially larger on exports than on imports. It is important to underline that this conclusion is based on the ceteris paribus assumption. On average, infrastructural investment in a certain country may only be expected to improve if no trading partners improve their infrastructures in similar proportions. Trade is a zero-sum game and the trade balance of an economy will only improve given that all economies in the rest of 
the world do not improve their infrastructures in similar proportions.

Moreover, there may also be structural asymmetries and intangible aspects adding to this difference in the exporter and importer infrastructure elasticities of trade. Infrastructure may be tailored more towards exports and not be neutral to the direction of trade. Even if the quality and stock of infrastructure is identical, the way it is utilized may differ between the incoming and outgoing traffic of goods. Differences between the two functions of the same infrastructure can be due to choices such as the amount of personnel allocated or prices charged for infrastructure utilization. Political factors may be another possibility that causes this asymmetry. If exporters politically have more lobbying power than importers, new infrastructure approved by governments may be biased to benefit exporters more than importers. The literature would therefore benefit from further research on microeconomic mechanisms that yield the "stylized facts" that we have uncovered in this meta-analysis.

Finally, our research provides crucial synthesized evidence for developing economies or even low-income economies where infrastructure deprivation is a fact. For instance, the 2005 report of the Commission for Africa emphasizes the need of a functioning transport and communications system for Africa and states that the continent's transport costs "local, national, and international - are around twice as high as those for a typical Asian country" and "to improve its capacity to trade Africa needs to make changes internally. It must improve its transport infrastructure to make goods cheaper to move" (Commission for Africa, 2005, p.14, 102). Our meta-analytic evidence adds useful evidence to back the argument that areas with poor infrastructure, such as parts of Africa, could greatly benefit from trade-enhancing infrastructure oriented policy measures. 


\section{A Appendix}

TABle 3.A.1

RoBUSTNESS ANALYSIS

\begin{tabular}{|c|c|c|c|c|}
\hline & \multicolumn{2}{|c|}{$\begin{array}{l}\text { OLS on deviations } \\
\text { from the mean }\end{array}$} & \multicolumn{2}{|c|}{$\begin{array}{l}\text { WLS on deviations } \\
\text { from the mean }\end{array}$} \\
\hline & $\begin{array}{c}\text { Exporter } \\
\text { Infrastructure }\end{array}$ & $\begin{array}{c}\text { Importer } \\
\text { Infrastructure }\end{array}$ & $\begin{array}{c}\text { Exporter } \\
\text { Infrastructure }\end{array}$ & $\begin{array}{c}\text { Importer } \\
\text { Infrastructure }\end{array}$ \\
\hline Methodology & & & & \\
\hline $\begin{array}{l}\text { Model accounts for zero trade selec- } \\
\text {-tion (Heckman, Tobit, Probit) }\end{array}$ & $\begin{array}{c}-0.104 \\
(0.0882)\end{array}$ & $\begin{array}{c}-0.459^{* *} \\
(0.193)\end{array}$ & $\begin{array}{l}-0.0367 \\
(0.0398)\end{array}$ & $\begin{array}{c}-0.890 * * \\
(0.310)\end{array}$ \\
\hline $\begin{array}{l}\text { Model accounts for endogeneity } \\
\text { (IV-based estimation) }\end{array}$ & $\begin{array}{c}0.362^{* * *} \\
(0.124)\end{array}$ & $\begin{array}{l}0.0267 \\
(0.180)\end{array}$ & $\begin{array}{l}-0.0718 \\
(0.0867)\end{array}$ & $\begin{array}{l}-0.0179 \\
(0.0110)\end{array}$ \\
\hline Gravity model & $\begin{array}{l}-0.188 \\
(0.383)\end{array}$ & & $\begin{array}{c}0.777 \\
(0.708)\end{array}$ & \\
\hline The point at which trade is mea & sured & & & \\
\hline Dependent variable is exports & $\begin{array}{l}0.324^{* *} \\
(0.161)\end{array}$ & $\begin{array}{l}-0.151 \\
(0.230)\end{array}$ & $\begin{array}{c}0.765^{* * *} \\
(0.139)\end{array}$ & $\begin{array}{c}0.118 \\
(0.214)\end{array}$ \\
\hline Infrastructure category & & & & \\
\hline Land transport infrastructure & $\begin{array}{l}0.194^{* *} \\
(0.0887)\end{array}$ & $\begin{array}{c}0.112 \\
(0.133)\end{array}$ & $\begin{array}{l}0.0540 \\
(0.109)\end{array}$ & $\begin{array}{c}0.181 \\
(0.117)\end{array}$ \\
\hline Maritime or air infrastructure & $\begin{array}{c}-0.000187 \\
(0.100)\end{array}$ & $\begin{array}{c}0.104 \\
(0.173)\end{array}$ & $\begin{array}{c}0.0960 \\
(0.0821)\end{array}$ & $\begin{array}{c}0.101 \\
(0.1000)\end{array}$ \\
\hline $\begin{array}{l}\text { Communication infrastructure } \\
\text { Composite measure (index) }\end{array}$ & $\begin{array}{l}0.0491 \\
(0.102)\end{array}$ & $\begin{array}{l}0.0377 \\
(0.125)\end{array}$ & $\begin{array}{c}0.0754 \\
(0.0885)\end{array}$ & $\begin{array}{c}0.0307 \\
(0.0896)\end{array}$ \\
\hline $\begin{array}{l}\text { Development level of the econ } \\
\text { which infrastructure is located }\end{array}$ & Reference dummy & & & \\
\hline Developing economy & $\begin{array}{l}0.208^{* *} \\
(0.0821)\end{array}$ & $\begin{array}{c}-0.0880 \\
(0.200)\end{array}$ & $\begin{array}{c}0.0501 \\
(0.0648)\end{array}$ & $\begin{array}{c}0.0538 \\
(0.0715)\end{array}$ \\
\hline Developed economy & $\begin{array}{l}0.0896 \\
(0.235)\end{array}$ & $\begin{array}{l}0.0456 \\
(0.206)\end{array}$ & $\begin{array}{l}-0.158 \\
(0.202)\end{array}$ & $\begin{array}{c}-0.0265^{*} \\
(0.0141)\end{array}$ \\
\hline
\end{tabular}


TABle 3.A.1

RoBUstness ANALYSIS (CONT'D)

\begin{tabular}{|c|c|c|c|c|}
\hline & \multicolumn{2}{|c|}{$\begin{array}{c}\text { OLS on deviations } \\
\text { from the mean }\end{array}$} & \multicolumn{2}{|c|}{$\begin{array}{l}\text { WLS on deviations } \\
\text { from the mean }\end{array}$} \\
\hline & $\begin{array}{c}\text { Exporter } \\
\text { Infrastructure } \\
\end{array}$ & $\begin{array}{c}\text { Importer } \\
\text { Infrastructure } \\
\end{array}$ & $\begin{array}{c}\text { Exporter } \\
\text { Infrastructure }\end{array}$ & $\begin{array}{c}\text { Importer } \\
\text { Infrastructure }\end{array}$ \\
\hline $\begin{array}{l}\text { Both types of economies } \\
\text { (mixed sample) }\end{array}$ & Reference dummy & & & \\
\hline Sample structure & & & & \\
\hline Sub-national or firm level & $\begin{array}{c}0.248 \\
(0.256)\end{array}$ & $\begin{array}{l}-0.584^{*} \\
(0.332)\end{array}$ & $\begin{array}{l}-0.0829 \\
(0.649)\end{array}$ & $\begin{array}{l}-0.713^{*} \\
(0.377)\end{array}$ \\
\hline No cross-section & $\begin{array}{l}0.0339 \\
(0.124)\end{array}$ & $\begin{array}{c}0.197 \\
(0.156)\end{array}$ & $\begin{array}{l}0.226^{*} \\
(0.119)\end{array}$ & $\begin{array}{l}0.259^{*} \\
(0.138)\end{array}$ \\
\hline Model specification & & & & \\
\hline Constrained model & $\begin{array}{l}0.0584 \\
(0.192)\end{array}$ & $\begin{array}{l}0.738^{*} \\
(0.441)\end{array}$ & $\begin{array}{c}0.312 \\
(0.216)\end{array}$ & $\begin{array}{c}0.371 \\
(0.385)\end{array}$ \\
\hline $\begin{array}{l}\text { Estimation excludes other } \\
\text { infrastructure categories }\end{array}$ & $\begin{array}{l}-0.0766 \\
(0.164)\end{array}$ & $\begin{array}{c}0.263 \\
(0.225)\end{array}$ & $\begin{array}{c}0.144 \\
(0.129)\end{array}$ & $\begin{array}{c}0.208 \\
(0.216)\end{array}$ \\
\hline $\begin{array}{l}\text { Model does not control for } \\
\text { transit or partner infrastructure }\end{array}$ & $\begin{array}{l}-0.137 \\
(0.214)\end{array}$ & $\begin{array}{c}1.255^{* * *} \\
(0.448)\end{array}$ & $\begin{array}{l}-0.104 \\
(0.186)\end{array}$ & $\begin{array}{l}0.962^{* *} \\
(0.339)\end{array}$ \\
\hline $\begin{array}{l}\text { Equation excludes multilateral } \\
\text { resistances }\end{array}$ & $\begin{array}{l}-0.0337 \\
(0.152)\end{array}$ & $\begin{array}{c}0.149 \\
(0.236)\end{array}$ & $\begin{array}{l}0.0469 \\
(0.200)\end{array}$ & $\begin{array}{l}-0.104 \\
(0.245)\end{array}$ \\
\hline Equation excludes income & $\begin{array}{l}-0.352 \\
(0.343)\end{array}$ & & $\begin{array}{c}0.349 \\
(0.665)\end{array}$ & \\
\hline $\begin{array}{l}\text { Tariffs or trade agreements } \\
\text { not considered }\end{array}$ & $\begin{array}{c}-0.395^{* * *} \\
(0.138)\end{array}$ & $\begin{array}{l}-0.0598 \\
(0.167)\end{array}$ & $\begin{array}{c}0.101 \\
(0.0760)\end{array}$ & $\begin{array}{c}0.0605^{* *} \\
(0.0247)\end{array}$ \\
\hline $\begin{array}{l}\text { Equation excludes } \\
\text { spatial/geographic variables }\end{array}$ & $\begin{array}{c}0.122 \\
(0.124)\end{array}$ & $\begin{array}{l}-0.191 \\
(0.133)\end{array}$ & $\begin{array}{l}-0.192 \\
(0.247)\end{array}$ & $\begin{array}{c}-0.152 \\
(0.0916)\end{array}$ \\
\hline $\begin{array}{l}\text { Equation excludes education } \\
\text { and human capital }\end{array}$ & $\begin{array}{l}0.0240 \\
(0.160)\end{array}$ & $\begin{array}{c}-1.276^{* * *} \\
(0.465)\end{array}$ & $\begin{array}{c}0.614^{* * *} \\
(0.121)\end{array}$ & $\begin{array}{c}-1.044^{* * *} \\
(0.270)\end{array}$ \\
\hline Population not considered & $\begin{array}{c}0.124 \\
(0.0911)\end{array}$ & $\begin{array}{l}0.0224 \\
(0.143)\end{array}$ & $\begin{array}{c}0.0330 \\
(0.0811)\end{array}$ & $\begin{array}{c}0.0188 \\
(0.0430)\end{array}$ \\
\hline $\begin{array}{l}\text { Governance variable(s) not } \\
\text { considered }\end{array}$ & $\begin{array}{c}-0.406^{* * *} \\
(0.107)\end{array}$ & $\begin{array}{l}-0.271 \\
(0.237)\end{array}$ & $\begin{array}{c}0.0216 \\
(0.0667)\end{array}$ & $\begin{array}{c}-0.458^{* *} \\
(0.187)\end{array}$ \\
\hline
\end{tabular}


TABLE 3.A.1

Robustness AnAlysis (CONT'D)

\begin{tabular}{|c|c|c|c|c|}
\hline & \multicolumn{2}{|c|}{$\begin{array}{l}\text { OLS on deviations } \\
\text { from the mean }\end{array}$} & \multicolumn{2}{|c|}{$\begin{array}{l}\text { WLS on deviations } \\
\text { from the mean }\end{array}$} \\
\hline & $\begin{array}{c}\text { Exporter } \\
\text { Infrastructure } \\
\end{array}$ & $\begin{array}{c}\text { Importer } \\
\text { Infrastructure }\end{array}$ & $\begin{array}{c}\text { Exporter } \\
\text { Infrastructure }\end{array}$ & $\begin{array}{c}\text { Importer } \\
\text { Infrastructure }\end{array}$ \\
\hline Equation excludes exchange rate & $\begin{array}{c}0.316^{* * *} \\
(0.114)\end{array}$ & $\begin{array}{l}0.0161 \\
(0.151)\end{array}$ & $\begin{array}{c}0.123^{*} \\
(0.0612)\end{array}$ & $\begin{array}{c}0.0225 \\
(0.0247)\end{array}$ \\
\hline $\begin{array}{l}\text { Equation excludes colonial, } \\
\text { cultural, or linguistic relations }\end{array}$ & $\begin{array}{l}0.00978 \\
(0.193)\end{array}$ & $\begin{array}{c}0.184 \\
(0.169)\end{array}$ & $\begin{array}{l}-0.0238 \\
(0.118)\end{array}$ & $\begin{array}{l}-0.0107 \\
(0.0858)\end{array}$ \\
\hline Nature of publication & & & & \\
\hline Highly ranked journals & $\begin{array}{l}0.00919 \\
(0.158)\end{array}$ & $\begin{array}{l}0.692^{*} \\
(0.377)\end{array}$ & $\begin{array}{c}0.307 \\
(0.200)\end{array}$ & $\begin{array}{c}0.290 \\
(0.287)\end{array}$ \\
\hline Advocacy & $\begin{array}{c}0.151 \\
(0.152)\end{array}$ & $\begin{array}{l}0.825^{* *} \\
(0.399)\end{array}$ & $\begin{array}{l}0.382^{* *} \\
(0.155)\end{array}$ & $\begin{array}{c}0.434 \\
(0.285)\end{array}$ \\
\hline Constant & $\begin{array}{l}0.329^{* * *} \\
(0.0272)\end{array}$ & $\begin{array}{c}0.365^{* * *} \\
(0.103)\end{array}$ & $\begin{array}{c}0.394^{* * *} \\
(0.0600)\end{array}$ & $\begin{array}{l}0.312^{* * *} \\
(0.0910)\end{array}$ \\
\hline R-squared & 0.413 & 0.333 & 0.584 & 0.769 \\
\hline Observations & 237 & 142 & 237 & 142 \\
\hline
\end{tabular}

Standard errors in parentheses

${ }^{*} p<0.10,{ }^{* *} p<0.05,{ }^{* * *} p<0.01$ 


\section{CHAPTER 4}

\section{Infrastructure and the International Export Performance of Turkish Regions ${ }^{1}$}

\subsection{Introduction}

Since the early 2000's, Turkey has been ambitiously investing in its transportation infrastructure. This period of increase in infrastructure investment has been accompanied by a surge in export performance, but at the same time has been coinciding with an increase in the current accounts deficit. Roughly at the same time with this upward trend in infrastructure investments and exports in Turkey, the economic literature focusing on the relationship between infrastructure and trade has also become enriched with many new research results. A positive effect of infrastructure on trade through the reduction of transport costs has been documented by Bougheas et al. (1999) for a sample of European countries; Limao and Venables (2001), Nordas and Piermartini (2004), Carrère (2006), Iwanow and Kirkpatrick (2009) and Portugal-Perez and Wilson (2012b) for samples consisting of a range of countries around the

\footnotetext{
${ }^{1}$ This chapter is the result of a joint work with Peter Nijkamp and Jacques Poot. The content of this chapter is identical to the forthcoming book chapter (co-authored by Peter Nijkamp and Jacques Poot) with the same title in The Region and Trade: new analytical directions edited by Amitrajeet A. Batabyal and Peter Nijkamp, World Scientific Publishing, 2015. Another version of this study is also available online as a UNU-MERIT working paper with serial number 2014-021, co-authored by Peter Nijkamp and Jacques Poot.
} 
world, Vijil and Wagner (2012) for a sample of developing countries, and in Chapter 3 of this thesis by means of meta-analysis, among others. In accordance with these results, the 2009 OECD/WTO report on aid for trade underlines the significance of infrastructure as "one of the most important regional public goods with enormous potential to facilitate cross-border trade, growth and development" (OECD/WTO, 2009).

A recent study on Turkey by Kustepeli et al. (2012) found no significant relationship between country-wide highway infrastructure ${ }^{2}$ and international trade. However, the authors also point out that there is a negative correlation between highway infrastructure investment and highway length. ${ }^{3}$ Considering that road infrastructure represents only a part of all trade-related infrastructure, ${ }^{4}$ and that infrastructure is not uniformly distributed among regions, it would be beneficial to focus on the infrastructure-trade relationship with explicit emphasis on different infrastructure categories and on the positioning of infrastructure in space. Therefore, this study aims to answer the following two questions: Firstly, how much of the recent expansion and the sub-regional differences in Turkey's export performance can be attributed to trade-related infrastructure? Secondly, how did the different types of infrastructure impact on exports? The answer to these questions can potentially provide some valuable information not only for Turkey but also for other emerging economies and LDC's where infrastructure deprivation remains as a crucial issue. Additionally, we note that many trade studies measure point infrastructure in terms of traffic (such as port or airport traffic). We aim to answer the above questions with a focus on capacity rather than traffic in order to reduce endogeneity concerns.

\footnotetext{
${ }^{2}$ The authors measure infrastructure interchangeably in two ways: the share of highway expenditures in the public budget, and highway length.

${ }^{3}$ We assume that a possible reason for their finding could be that investment could have been made for removing older, curvier roads, and replacing them with straighter roads (or for example, building a tunnel) which would reduce the length of a highway from one point to another.

${ }^{4}$ As of 2009, the distribution of Turkish exports by modes of transportation were as follows: Roads $41.7 \%$, Sea $46.0 \%$, Air $9.5 \%$, Rail $0.9 \%$, and other modes $1.8 \%$ (Karacadag Development Agency, 2011).
} 


\subsection{Theoretical Framework}

We follow the Anderson and van Wincoop (2003) (henceforth AvW) gravity model of trade, in which consumers in region $j$ maximize the following utility function:

$$
U_{j}=\left(\sum_{i=1}^{n} \beta_{i}^{\frac{1-\sigma}{\sigma}} c_{i j}^{\frac{\sigma-1}{\sigma}}\right)^{\frac{\sigma}{\sigma-1}}
$$

subject to:

$$
\sum_{i=1}^{n} c_{i j} \tau_{i j} p_{i}=y_{j}
$$

where $n$ is the number of regions, $\sigma$ is the elasticity of substitution, $c_{i j}$ is the consumption of the unique product of region $i$ by the consumers in region $j, \beta_{i}>0$ is a distribution parameter for determining the weight assigned by consumers in $j$ on the unique product of $i$, and the supply price of the exporter is $p_{i}$. AvW define $p_{i j}$ as the price for goods of region $i$ that the consumers in $j$ face. The supply price $p_{i}$ is scaled by a trade cost factor $\left(t_{i j}>1\right)$ that applies to the trade from $i$ to $j$ such that $p_{i} t_{i j}=p_{i j} .{ }^{5}$ Consequently, the budget constraint for region $j$ in the $\mathrm{AvW}$ model is $\sum_{i} c_{i j} t_{i j} p_{i}=y_{j}$. In our adaptation of the AvW model, we define $\tau_{i j}$ as the trade costs divided by the export capacity of region $i$ denoted by $s_{i}$ :

$$
\tau_{i j}=\frac{t_{i j}}{s_{i}}
$$

where $0<s_{i} \leq 1$. If $s_{i}$ is equal to 1 , then the export capacity of region $i$

${ }^{5}$ As noted in Chapter 3, the Anderson and van Wincoop (2003) model assumes frictionless intra-regional trade such that $t_{i i}=1$. 
is at a maximum, so that the c.i.f. price to consumers in region $j$ is at a minimum. Alternatively, if $s_{i}$ is close to zero, region $i$ has virtually no capacity to export and the cost of exporting from $i$ to $j$ would be prohibitively high. Therefore, exports from $i$ to $j$ are subject to two constraints: (1) the budget constraint of region $j$ and (2) the export capacity constraint of region $i$. The following market clearing condition is imposed:

$$
y_{j}=\sum_{i=1}^{n} x_{j i}
$$

where $x_{j i}$ is the value of exports of $j$ to $i$ and $p_{j} \tau_{j i} c_{j i}=x_{j i}$.

Equation (4.4) says that the income of region $j$ is the sum of all exports of $j$ to all other regions $i$. Solving the above defined model leads to the gravity equation:

$$
x_{i j}=\frac{y_{j} y_{i}}{y_{w}}\left(\frac{t_{i j} / s_{i}}{P_{j} P_{i}}\right)^{1-\sigma}
$$

where $y_{w}$ is the world income. A common proxy for $t_{i j}$ in trade literature is the distance between origin and destination economies. However, it has been emphasized by Beckerman (1956) that relative distance should be taken into consideration rather than absolute distance when examining the impact of distance on trade flows. Beckerman (1956) also suggested using prices in the origin and destination economies for measuring the relative distances. The author highlights two distance elements and defines them as follows: "the relative distance of every other country to the given country, which will influence the import pattern of the given country in one way" and "the relative distance of the given country to each other country, which will affect the export pattern of each other country and will thereby also have an effect on the import pattern of the given country" Beckerman (1956, p.36). In the Anderson and van Wincoop (2003) model this concept is refined as "multilateral resistance" and formalized as price indices in the terms $P_{j}$ and $P_{i}$ where 


$$
\begin{aligned}
& P_{j}^{1-\sigma}=\sum_{i=1}^{n}\left(\frac{\tau_{i j}}{P_{i}}\right)^{1-\sigma} \frac{y_{i}}{y_{w}} \\
& P_{i}^{1-\sigma}=\sum_{j=1}^{n}\left(\frac{\tau_{i j}}{P_{j}}\right)^{1-\sigma} \frac{y_{j}}{y_{w}}
\end{aligned}
$$

AvW assume symmetry of trade barriers $\left(\tau_{i j}=\tau_{j i}\right)$. This condition translates to our adaptation as $\frac{t_{i j}}{s_{i}}=\frac{t_{j i}}{s_{j}}$ which assumes that the trade costs which $i$ and $j$ face relative to their own export capacities are equal.

The log-linearization of (4.5) yields

$$
\begin{aligned}
\ln X_{i j}= & \ln y_{j}+\ln y_{i}-\ln y_{w}+(1-\sigma) \ln t_{i j} \\
& -(1-\sigma) \ln s_{i}-(1-\sigma) \ln P_{j}-(1-\sigma) \ln P_{i}
\end{aligned}
$$

Assuming that $\sigma>1$, the closer region $i$ is to its full capacity to export, the higher the export flows from $i$ to $j$ conditional on the level of income of the consumers in $j$.

Trade costs $t_{i j}$ are defined by Anderson and van Wincoop (2004) as

$$
t_{i j}=\prod_{m=1}^{M}\left(z_{i j}^{m}\right)^{\gamma_{m}}
$$

where $z_{i j}^{m}$ is the $m$ 'th observable factor for regions $i$ and $j$ that is associated with trade costs $t_{i j}$, and $\gamma_{m}$ is the parameter measuring the role of the $m$ 'th factor in trade costs. Substituting (4.9) into (4.8) gives 


$$
\begin{aligned}
\ln X_{i j}= & \ln y_{j}+\ln y_{i}-\ln y_{w}+(1-\sigma) \sum_{m=1}^{M} \gamma_{m} \ln z_{i j}^{m} \\
& +(\sigma-1) \ln s_{i}-(1-\sigma) \ln P_{j}-(1-\sigma) \ln P_{i}
\end{aligned}
$$

which yields our regression equation to be estimated after including an error term to the right hand side. Section 5.5 explicitly shows the variables corresponding to each term in equation (4.10).

\subsection{Data and Descriptive Statistics}

The system of reporting the regional output data for Turkey has gone through several modifications in the last decade. The spatial scale has been changed to NUTS 2 from NUTS $3^{6}$ and the indicator of output has been changed to gross value added from gross domestic product. This change in reporting also corresponds time-wise to a two year gap in the data between 2001 and 2004. Moreover, the regional public investments data are reported only if they are province (NUTS 3) specific, but not if an investment was directed to more than one NUTS 3 level province. To cope with these issues in the data, we use the adjusted NUTS 2 level GVA and public investment figures compiled in Chapter 5. To summarize, the adjusted series for both these variables account for the differences in spatial scale in the officially reported data by (1) aggregating NUTS 3 (provincial) figures to NUTS 2 (regional) ones, (2) adjusting for the change of measurement from GDP to GVA, and (3) imputing the two consecutive missing years. Chapter 5 also shows for a sample of three regions, that inflating the public investment figures by an estimated amount ${ }^{7}$ for each region in order to account for the unreported province-specific investments cause roughly a parallel shift

\footnotetext{
${ }^{6}$ NUTS stands for "Nomenclature of Units for Territorial Statistics."

${ }^{7}$ This estimation is the result of meticulous extraction of investment figures reported separately by project name with attached locational information.
} 
upwards in the investment trend lines for these regions and advocate that using the officially reported data should not cause biases.

Table 5.A.1 lists the 26 regions that form the basis of the empirical analysis. The boundaries of these regions are displayed in Figure 4.A.1. ${ }^{8}$ Table 5.A.3 presents the sources and definitions of the variables used in the estimations. We use a set of covariates that consist of both continuous and dummy variables. Several of the variables, especially the geographical ones and some of the infrastructure capacity variables are either completely time invariant, or only exhibit very small changes over time. In our estimations this causes some information to be lost or some coefficients to be estimated imprecisely if regional fixed effects are included. Additionally, some variables such as Air capacity and EU customs union are constant for certain regions or countries throughout the time range while some variation is present for other trading units. This implies that the fixed effects estimation would still keep such variables even though for many observations in the sample the figures would be absorbed by the fixed effects. Therefore, in our fixed effects estimations, we manually drop such "mostly constant" variables for consistency. We employ distance based variables as in Granato (2008) from regional cores to exit nodes as a component of transport costs. These variables are also constant since they are defined in terms of Euclidean distance rather than road distance.

The descriptive statistics of the variables in our panel are presented in Tables $4.2 \mathrm{a}$ and $4.2 \mathrm{~b}$ for region specific and general variables respectively. All summaries are based on observations for nine years. ${ }^{9}$ These tables show that for some regions certain types of air and, naturally, maritime infrastructure does not exist at all. The summarized variables in levels enter the econometric estimations in natural logarithms as suggested

\footnotetext{
${ }^{8}$ These regions are not administrative units but only statistical regions. The largest administrative sub-national units in Turkey are provinces. All regions consist of at least two provinces, except for Istanbul, Ankara, and Izmir which are provinces and regions at the same time.

${ }^{9}$ The continuous variables that enter the estimations in natural logarithms are transformed to $l n$ form after adding one if they include minimum values of zero in order to avoid values of $\ln (0)$.
} 
by the theoretical model presented in Section 4.2 (except the dummy variables).

Figures 4.1 to 4.3 show the trends in country-wide exports, public investments in transportation and communication, and gross value added respectively for the years 2002 through 2010. A general upwards trend can be observed for all three indicators. Figure 4.4 plots the relationship between exports and public investments in transportation and communication observed during the nine-year period. A positive association is present, however higher values of investment are not always corresponding to higher values of exports. This can be attributable to a possible satiation effect. In other words, after a certain point, investments and exports may no longer be strongly related. It is also important to note that no implication on causation can be made at this point. In order to explore the spatial dimension for these figures, we plot the regionally disaggregated trend lines for the five economically largest regions ${ }^{10}$ in natural logarithms. ${ }^{11}$ Figures 4.5 and 4.6 show the trends in exports and investments respectively for these regions. The largest economy of the country, Istanbul, is persistently higher than the other four largest economies in both exports and investments. While for exports smoother trend lines are observed, the trend lines for investments are rather erratic except for Istanbul. As the investment allocation is subject to central decision-making in Turkey where the decision-makers consider a range of factors before directing regional public investments (as elaborated in Chapter 5), relatively smooth trends would have implied that investment decisions are mostly unchanged from one year to another while this figure suggests otherwise.

The spatial variation in the export and investment values for the years 2002 and 2010 are presented in Figures 4.7a, 4.7b, 4.8a and 4.8b respectively. These maps which present a snapshot of the geographic distribution for the first and last years of the sample suggest that the rel-

\footnotetext{
${ }^{10}$ As of 2008 .

${ }^{11}$ We use the logarithmic transformation to avoid a large gap in the graph between the region with the highest GVA and the other four.
} 
ative export performances of the regions did not change drastically ${ }^{12,13}$ while for investments there is not such a persistence over time. It is also important to underline that the infrastructure investment figures may not coincide with the regional distribution of infrastructure stock. For a closer examination of the infrastructure-export relationship, Section 5.5 discusses the estimation of equation 4.10. All estimations are conducted after dropping importer partner countries that have zero imports through the covered nine years with 23 or more of the regions of Turkey. We are then left with 180 countries in our data set out of the original 186 countries. ${ }^{14}$

TABLE 4.1

VARIABLE DEFINITIONS

\begin{tabular}{|c|c|c|}
\hline Name & Year Coverage & Description \\
\hline Exports $_{i j, t}$ & $2002-2010$ & $\begin{array}{l}\text { Bilateral value of regional to- } \\
\text { tal exports in constant } 2005 \\
100,000 \text { USD. Source: Turk- } \\
\text { stat. }\end{array}$ \\
\hline $\begin{array}{l}\text { Regional } \\
\text { GVA } \\
\text { it }\end{array}$ & $2002-2010$ & $\begin{array}{l}\text { Regional gross value added con- } \\
\text { stant } 2005 \text { 100,000 USD. Mod- } \\
\text { ified as specified in Appendix. } \\
\text { Source: Turkstat }\end{array}$ \\
\hline $\begin{array}{l}\text { Country } \\
\text { GVA } A_{j t}\end{array}$ & $2002-2010$ & $\begin{array}{l}\text { Partner country gross value } \\
\text { added constant } 2005100,000 \\
\text { USD. Modified as specified in } \\
\text { Appendix. Source: UN. }\end{array}$ \\
\hline $\begin{array}{l}\text { Partner country ex- } \\
\text { change rate }_{j t}\end{array}$ & $2002-2010$ & $\begin{array}{l}\text { Partner country exchange rate } \\
\text { to USD. Source:Penn world ta- } \\
\text { ble } 7.1 \text { (Version } 2 \text { for China). }\end{array}$ \\
\hline
\end{tabular}

${ }^{12}$ Except for region TRC:3 in the Southeast.

${ }^{13}$ All maps have been made by using the SPMAP command in Stata developed by Pisati (2007). NUTS 3 provinces in the originally available shapefile have been aggregated to NUTS 2 regions using the Stata module MERGEPOLY developed by Picard and Stepner (2012).

${ }^{14}$ Among the six countries dropped, Sudan has been dropped due to missing years in the data. 
TABLE 4.1

VARIABLE DEFINITIONS (CONT'D)

\begin{tabular}{|c|c|c|}
\hline Name & Year Coverage & Description \\
\hline Distance $_{i j}$ & Constant & $\begin{array}{l}\text { Euclidean distance between } \\
\text { the most populous province } \\
\text { of the region and the cap- } \\
\text { ital of the partner country. } \\
\text { Source for region coordinates: } \\
\text { http://www.tageo.com/index- } \\
\text { e-tu-cities-TR.htm. Source for } \\
\text { country coordinates: UN. }{ }^{15}\end{array}$ \\
\hline $\begin{array}{l}\text { Land transport } \\
\text { infrastructure }_{i t}\end{array}$ & $2002-2010$ & $\begin{array}{l}\text { Index made from log highway, } \\
\text { road, and railroad lengths per } \\
1000 \text { square meters. Export } \\
\text { shares that go through these } \\
\text { type of infrastructure are used } \\
\text { as the weights. }\end{array}$ \\
\hline $\begin{array}{l}1+\text { Average minimum } \\
\text { euclidean distance to } \\
\text { major airports }\end{array}$ & constant & $\begin{array}{l}\text { Regional average distance of } \\
\text { each provincial center to the } \\
\text { closest major airport. }\end{array}$ \\
\hline $\begin{array}{l}1+\text { Average minimum } \\
\text { euclidean distance to } \\
\text { major ports }{ }_{i t}\end{array}$ & constant & $\begin{array}{l}\text { Regional average distance of } \\
\text { each provincial center to the } \\
\text { closest major port. }\end{array}$ \\
\hline Handling capacity it & $\begin{array}{l}\text { constant } \\
\text { (as of } 2006)\end{array}$ & $\begin{array}{l}\text { Port handling capacity in ma- } \\
\text { jor ports in } 100,000 \text { tons (Gen- } \\
\text { eral cargo and dry bulk). }\end{array}$ \\
\hline Air capacity $i t$ & $2002-2010$ & $\begin{array}{l}\text { Total passenger capacity (in } \\
100,000 \text { persons) of the region's } \\
\text { airports. Compiled from the } \\
\text { information on area and estab- } \\
\text { lishment dates available at sev- } \\
\text { eral websites of the Republic of } \\
\text { Turkey: Ministry of Transport, } \\
\text { Maritime Affairs and Commu- } \\
\text { nication. }\end{array}$ \\
\hline Similar language $_{i j}$ & constant & $\begin{array}{l}\text { Equals to } 1 \text { if parner country } \\
\text { has a Turkic language as an of- } \\
\text { ficial language, zero otherwise. }\end{array}$ \\
\hline
\end{tabular}

${ }^{15}$ The center of a region is taken as its most populous city of the region in 2008 (according to the Turkish statistical office's query pages at www.turkstat.gov.tr). except for Agri (Agri is from www.wikipedia.com). Generated from coordinates using Stata command SPMAT (Drukker et al., 2011). MRT corrected as suggested by Baier and Bergstrand (2009). 
TABle 4.1

VARIABLE DEFINITIONS (CONT'D)

\begin{tabular}{|c|c|c|}
\hline Name & Year Coverage & Description \\
\hline EU customs union $_{j t}$ & $2002-2010$ & $\begin{array}{l}\text { Dummy }=1 \text { if the partner } \\
\text { country is a EU member, there- } \\
\text { fore being in customs union } \\
\text { with Turkey. }\end{array}$ \\
\hline DSL lines $s_{i t}$ & $2002-2010$ & $\begin{array}{l}\text { Table Number of DSL/ADSL } \\
\text { lines in the PTT offices per } \\
1000 \text { persons. Source: Repub- } \\
\text { lic of Turkey - General Direc- } \\
\text { torate of PTT. Values for } 2002 \\
\text { and } 2005 \text { are imputed as shown } \\
\text { in appendix. }\end{array}$ \\
\hline $\begin{array}{l}\text { Importer population }{ }_{j t} \\
(1000 \text { persons) }\end{array}$ & $2002-2010$ & 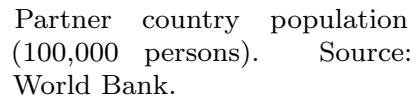 \\
\hline $\begin{array}{l}\text { Exporter population } \\
(1000 \text { persons })_{i t}\end{array}$ & $2002-2010$ & $\begin{array}{l}\text { Regional population }(100,000 \\
\text { persons). Source: OECDstat. }\end{array}$ \\
\hline Common open border ${ }_{i j}$ & constant & $\begin{array}{l}\text { Equals to } 1 \text { if the region and } \\
\text { the partner country share a } \\
\text { common border that is open to } \\
\text { trade, zero otherwise. }\end{array}$ \\
\hline $\begin{array}{l}\text { Partner country is } \\
\text { landlocked }_{j}\end{array}$ & constant & $\begin{array}{l}\text { Equals to } 1 \text { if the partner coun- } \\
\text { try is landlocked, zero other- } \\
\text { wise. }\end{array}$ \\
\hline
\end{tabular}


TABLE 4.2

Summary STATISTICS

(A) For exporting REgions

\begin{tabular}{|c|c|c|c|c|c|}
\hline Variable & Mean & Std. Dev. & Min. & Max. & $\mathbf{N}$ \\
\hline Regional Exports ${ }_{i t}$ & 1764.69 & 5156.16 & 0 & 47666.66 & 1620 \\
\hline Regional (Exporter) GVA $i t$ & 133058.42 & 178652.48 & 19622.32 & 1072149.75 & 234 \\
\hline Land transport infrastructure $i t$ & 75.73 & 17.57 & 50.78 & 139.82 & 234 \\
\hline DSL lines per cap.it & 0.046 & 0.038 & 0.0005 & 0.31 & 234 \\
\hline Air capacityit & 35.03 & 59.92 & 0 & 285 & 234 \\
\hline Public port capacity ${ }_{i}$ & 6.57 & 11.21 & 0 & 32.47 & 234 \\
\hline Number of private ports ${ }_{i}$ & 2.42 & 5.36 & 0 & 27 & 234 \\
\hline Avg. min. dist. to major airports $s_{i}$ & 3.61 & 3.08 & 0.14 & 10.24 & 234 \\
\hline Avg. min. dist. to major ports ${ }_{i}$ & 2.40 & 1.85 & 0.09 & 6.98 & 234 \\
\hline Region (exporter) Population ${ }_{i t}$ & 27.32 & 20.36 & 7.33 & 129.15 & 234 \\
\hline
\end{tabular}

(B) For IMPorting COUNTRIES

\begin{tabular}{|c|c|c|c|c|c|}
\hline Variable & Mean & Std. Dev. & Min. & Max. & $\mathbf{N}$ \\
\hline Country (importer) GVA $A_{j t}$ & 2379450.86 & 10587286.74 & 955.01 & 132041000 & 1620 \\
\hline distance $_{i j}$ & 5897.20 & 4061.41 & 449.86 & 17401.78 & 1620 \\
\hline MRT corrected dist.ij & 0.0002 & 0.00004 & 0.0001 & 0.0003 & 1620 \\
\hline Exchange rate $_{i j, t}$ & 384.62 & 1486.86 & 0.19 & 21226.34 & 1548 \\
\hline EU customs union $_{i j, t}$ & 0.11 & 0.32 & 0 & 1 & 1620 \\
\hline Similar language $_{i j}$ & 0.028 & 0.16 & 0 & 1 & 1620 \\
\hline Country (importer) Population ${ }_{j t}$ & 345.41 & 1337.89 & 0.28 & 13378.25 & 1620 \\
\hline landlocked importer $_{j}$ & 0.22 & 0.42 & 0 & 1 & 1620 \\
\hline Common open border ${ }_{i j}$ & 0.028 & 0.16 & 0 & 1 & 1620 \\
\hline
\end{tabular}


Figure 4.1

Exports, TURKey (CONSTANT 2005 MilLions OF USD).

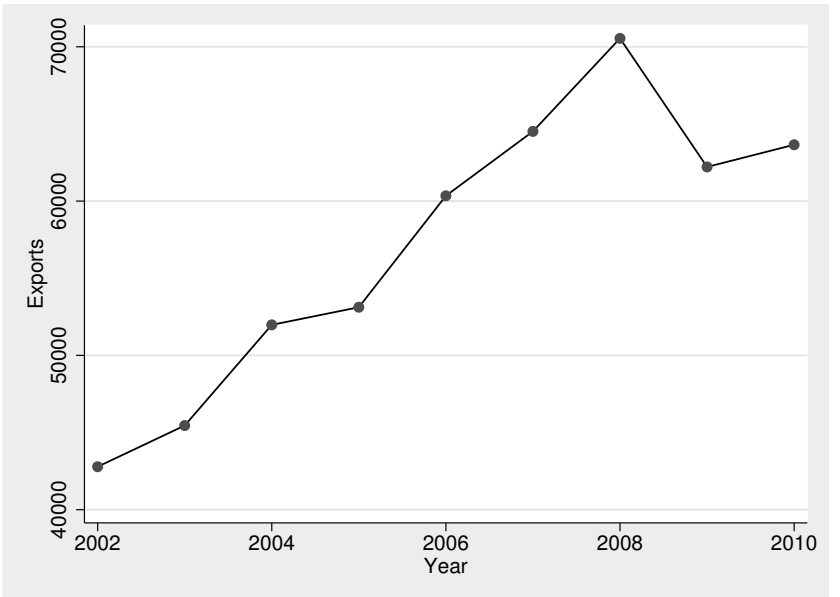

FigURE 4.2

PUblic inVESTMENTS IN TRANSPORTATION AND COMMUNICATION, TURKEy (CONSTANT 2005 Millions OF USD).

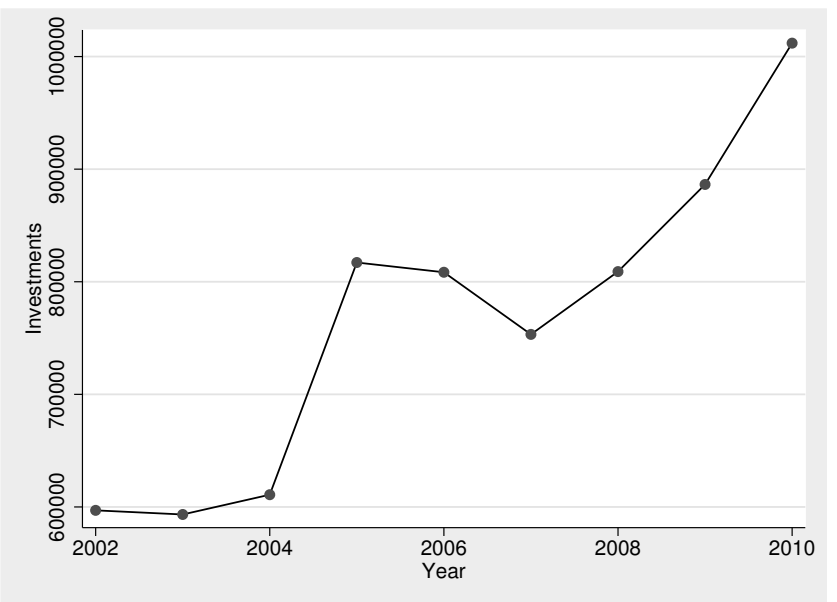


Figure 4.3

Gross value Added, Turkey (CONStant 2005 Billions of USD).

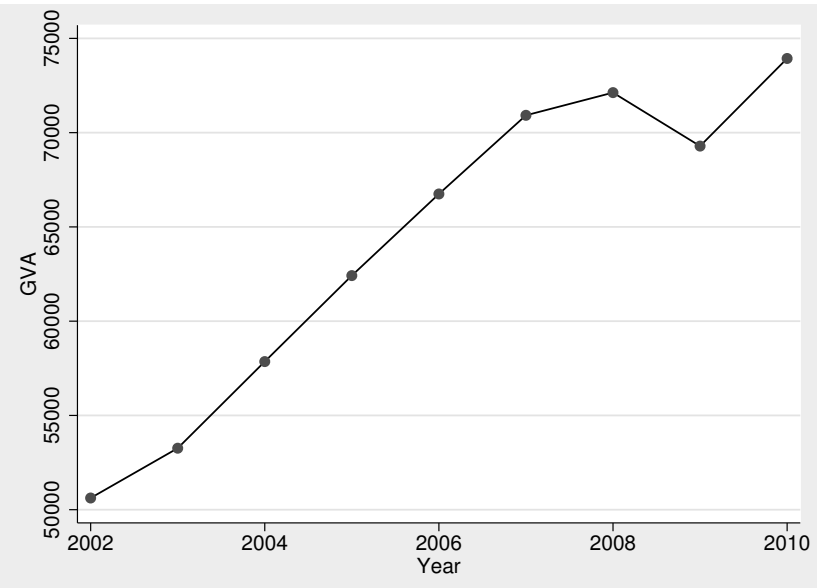

Figure 4.4

EXPORTS AND PUBLIC INVESTMENT IN TRANSPORTATION AND COMMUNiCATION, TURKEY (CONSTANT 2005 Millions USD).

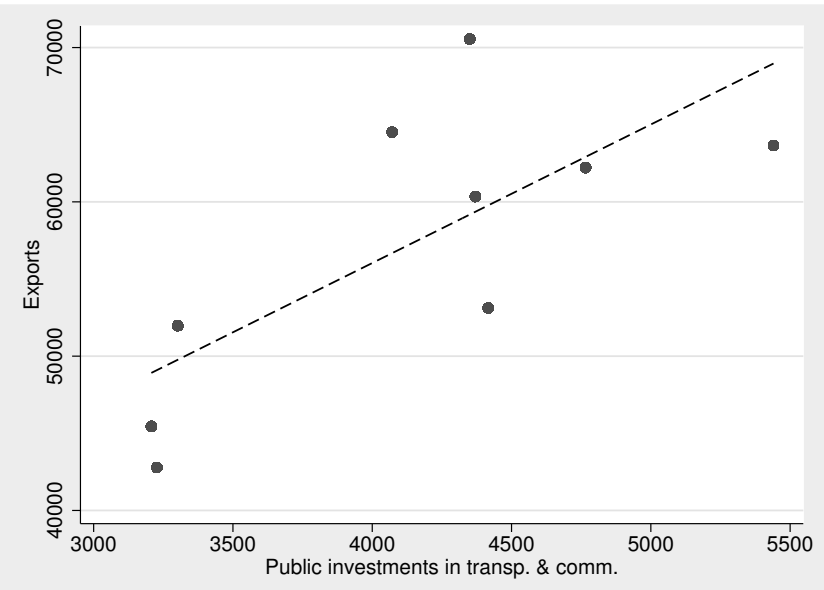


Figure 4.5

THE NATURAL LOGARITHM OF REGIONAL EXPORTS FOR THE FIVE LARGEST REgIONS, TURKEY (CONSTANT 2005 Millions OF USD).

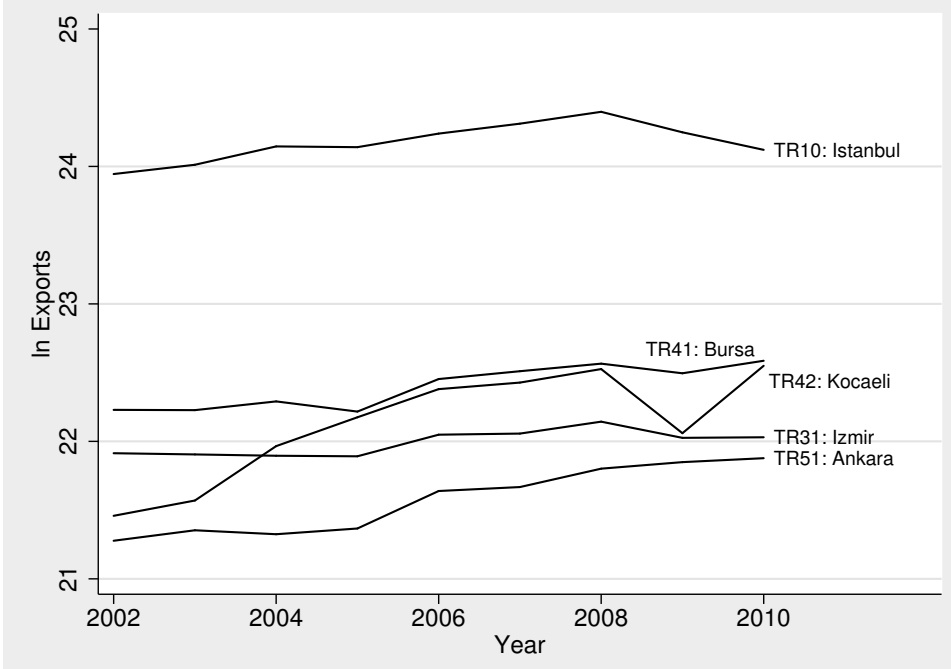


FiguRE 4.6

ThE NATURAL LOGARITHM OF PUBLIC INVESTMENTS IN TRANSPORTATION AND COMMUNICATION FOR THE FIVE LARGEST REGIONS, TURKEY (CONSTANT 2005 MILLIONS OF USD).

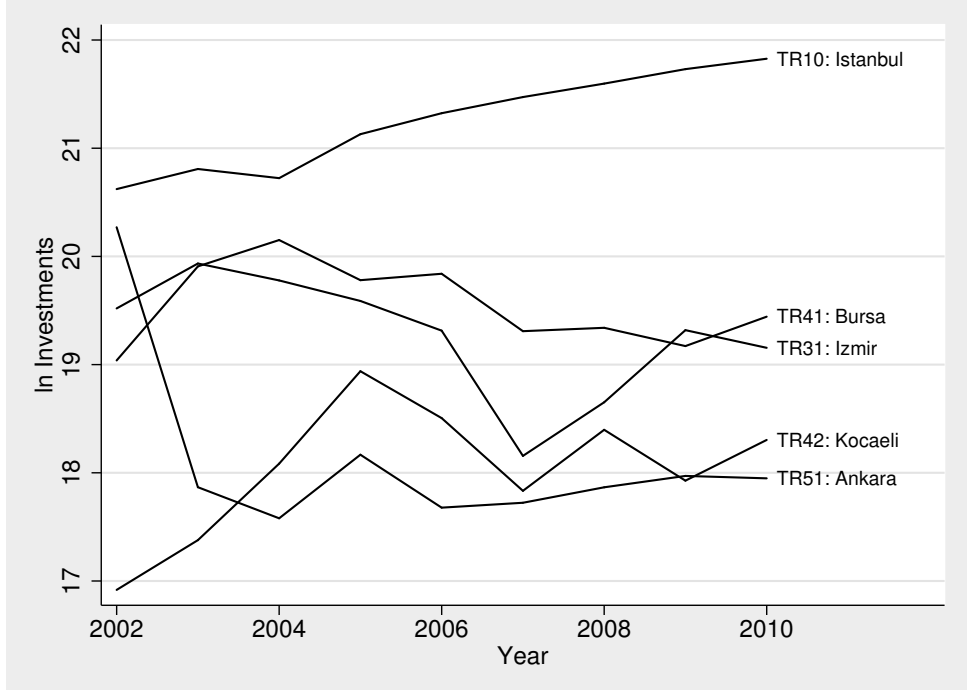


Figure 4.7

REgIONAL EXPORTS

(A) 2002

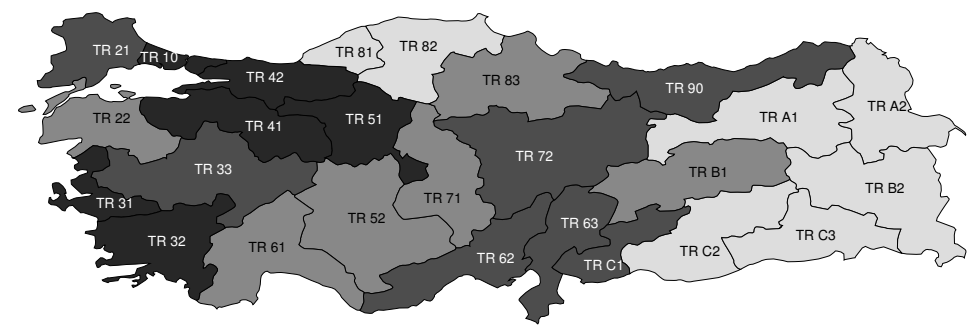

$(8.30 \mathrm{e}+08,2.51 \mathrm{e}+10$
$(3.22 \mathrm{e}+08,8.30 \mathrm{e}+08$

$\square(5.44 \mathrm{e}+07,3.22 \mathrm{e}+08]$
$\square[1453557,5.44 \mathrm{e}+07]$

(B) 2010

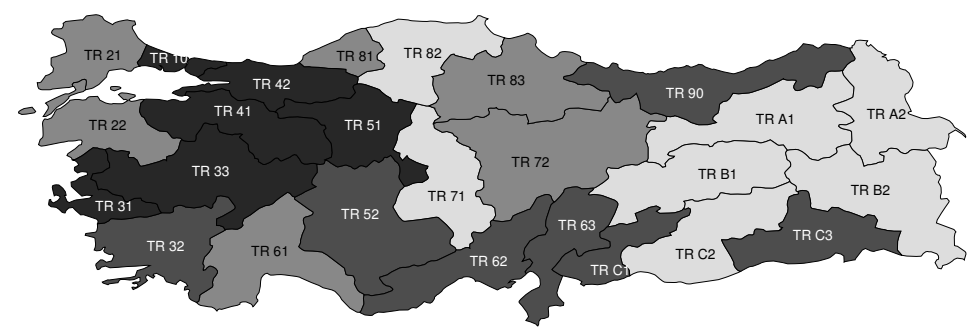

Q $(2.07 e+09,2.99 e+10)$

$\square$
$\square[2.47 \mathrm{e}+07,2.03 \mathrm{e}+08]$ 
FiguRE 4.8

Public investment in tRANSPORTATION AND COMMUNICATION

(A) 2002

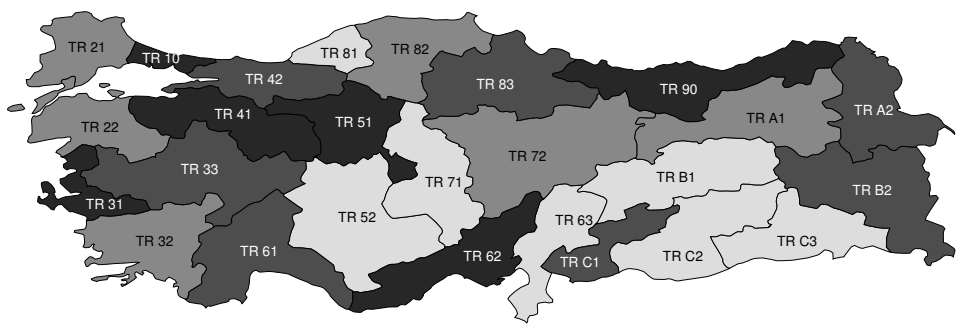

Q $(5.60 e+07,9.04 e+08$

口 $(2.11 \mathrm{e}+07,5.60 \mathrm{e}+07]$

口[953423.6, 6742067]

(в) 2010

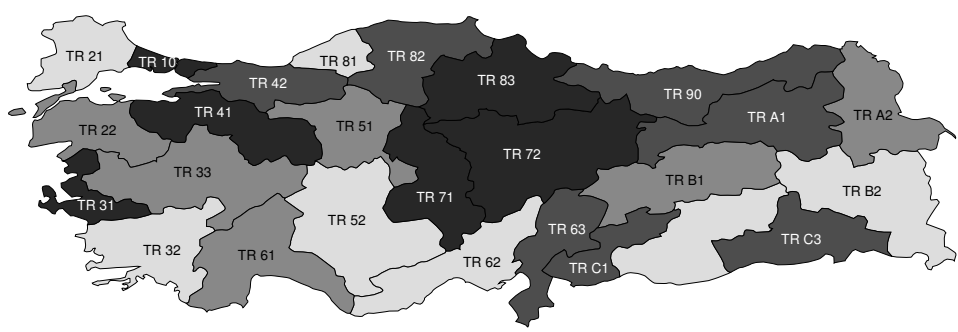

(1.27e+08, 3.02e+09]

$\square(4.89 e+07,7.53 e+07$
$\square[1.52 e+07,4.89 e+07]$ 


\subsection{Estimation and Empirical Results}

There are two methods that are common in the trade literature for controlling for the unobservable multilateral resistance terms (MRT) $P_{j}$ and $P_{i}$, namely by estimating the gravity model with importer and exporter fixed effects or by implementing the approach suggested by Baier and Bergstrand (2009) for approximating the MRT's expressed as price indices in equation (4.5). The Baier and Bergstrand (2009) method is adapted to our split-sample (non-symmetric trade matrix) case for the distance variable as follows; replacing their GDP-share weighted averages with simple averages (i.e. there is no GDP weighting of the variables in our study), we adjust the distance variable for MRT's as: In MRT adjusted distance ${ }_{i j}=$ lnDistance $_{i j}-\left(\frac{1}{n}\right) \sum_{j=1}^{n}$ lnDistance $_{i j}-$ $\left(\frac{1}{m}\right) \sum_{i=1}^{m} \operatorname{lnDistance~}_{i j}+\left(\frac{1}{m n}\right) \sum_{i=1}^{m} \sum_{j=1}^{n}$ lnDistance $_{i j}$ where $n$ is the number of importing countries and $\mathrm{m}$ is the number of exporting regions. The first term on the right-hand-side is the unadjusted $l n$ distance of the exporting region $i$ to the importing country $j$, the second term is the average $l n$ distance of the exporting region $i$ to all importing countries, the third term is the average $\ln$ distance of all exporting regions to the importing country $j$, and the fourth term is the average $l n$ distance of all exporting regions to all importing countries. Additionally, if a random distribution of multilateral resistances is assumed, the specification can be estimated using a random effects model (Shepherd, 2012). We use all three approaches in our estimations. However, a pair random effect estimation is ruled out in favor of fixed effects estimation by the Hausman test statistic reported in Table 4.4. We define the terms in equation (4.10) as functions of several empirical variables as follows:

$$
\begin{aligned}
& X_{i j}=\text { Exports }_{i j} \\
& y_{i}=\text { Region (exporter) } G V A_{i} \\
& z_{i j}^{m}=f\left(\text { Distance }_{i j} \text {, Average minimum distance to major airports } s_{i},\right. \\
& \text { Average minimum distance to major ports }{ }_{i}, \text { Common open border }{ }_{i j} \text { ) } \\
& s_{i}=g\left(\text { Land transport infrastructure }{ }_{i} \text {, Public port capacity }{ }_{i},\right. \\
& \text { Air capacity, DSL lines per capita } \left.a_{i t}\right)
\end{aligned}
$$


As the parameter $\sigma$ is associated with the terms $\sum_{m}^{M} \gamma_{m} l n z_{i j}$ and $\ln s_{i}$ in equation (4.10), and these terms are determined by a multitude of empirically specified variables, we do not attempt an explicit estimation of this model parameter. In order to augment the theoretical gravity model with variables that account for region and country characteristics and their connectedness, the following variables are added to the specification: Partner country exchange rate $_{j}$, Similar language ${ }_{i j}$, EU customs union $_{i j}$, Country (importer) population ${ }_{j}$, Regional (exporter) population ${ }_{i}$, and Partner country is landlocked ${ }_{j}$.

After including the time index $t$, constant and error terms, the final specification is:

$$
\begin{aligned}
& \ln _{\text {Exports }_{i j, t}} \\
& =a+\beta_{1} \text { lnRegional (exporter) } G V A_{i t} \\
& +\beta_{2} \operatorname{lnCountry} \text { (importer) GV } A_{j t}+\beta_{3} \ln \text { Distance }_{i j} \\
& +\beta_{4} \ln (\text { Partner country exchange rate })_{j t} \\
& +\beta_{5} \text { EU } \text { customs union }_{j t}+\beta_{6} \text { Similar language }_{j} \\
& +\beta_{7} \text { Land transport infrastructure }{ }_{i t} \\
& +\beta_{8} \ln D S L \text { per capita } i t+\beta_{9} \ln \text { Air capacity } \text { cit }_{1} \\
& +\beta_{10} \text { lnPublic port capacity } i+\beta_{11} \text { Number of private ports } s_{i} \\
& +\beta_{12} \text { lnAverage minimum distance to major airports } s_{i} \\
& +\beta_{13} \text { lnAverage minimum distance to major ports }{ }_{i} \\
& +\beta_{14} \text { lnRegional (exporter) population } i t \\
& +\beta_{15} \text { lnCountry (importer) population }{ }_{j t} \\
& +\beta_{16} \text { Partner country is landlocked } d_{j} \\
& + \text { Common open border }{ }_{i j}+\epsilon_{i j, t}
\end{aligned}
$$

Table 4.3 presents estimation results of pooled OLS models with and without year dummies, and of an OLS model with MRT adjusted distance variable. Additionally, because in our data set 19,379 observations of zero exports exist, which is 46 percent of all trade flows, we also report the results of a Heckman sample selection model developed by Heckman 
(1979) to cope with possible selection bias. ${ }^{16}$ We hypothesize that the adjacency of two economies would increase their probability to trade, but would not impact the amount of trade once trade has begun. Therefore, the variable Common open border $r_{i j}$ is the only selection term we employ. ${ }^{17}$ We separately discuss this estimation in (Section 4.A).

\section{Transport costs and export capacity}

Our results highlight the importance of air transportation infrastructure in enhancing export performance. In Aircapacity is significant and positive in all models at Tables 4.3 and 4.4. As mentioned earlier, we include the variables that are measured in terms of distance as proxies for the trade costs $t_{i j}$. Therefore, role of air transport infrastructure accessibility is also examined by the variable $\ln$ (Avg. min distance to airports). This variable yields negative but insignificant results in our estimations; we do not observe conclusive evidence that economies which are further away from airports export less than those that are closer to these exit nodes, but we find robust evidence that the transport capacity of these nodes are important.

Port infrastructure related variables present interesting results. While In Public port capacity is negative and significant in all our models, Number of private ports yields positive and significant coefficients. This could be a sign of a crowding-out effect, or could underline that regions with more privately operated ports are more efficient than those endowed with mostly public ports. The results also show that distance to ports has a negative impact on regional exports, further highlighting the importance of this infrastructure type in export performance. This observed difference in the role of distance for airports and ports is easy to explain; distance would matter for ports because of bulky and heavy goods being transported, but not for airports because of high-value but small-volume exports.

\footnotetext{
${ }^{16}$ These results are presented in Table 4.A.1 in the annex.

${ }^{17}$ The Heckman model is estimated by maximum likelihood.
} 
The land infrastructure index is associated significantly and positively with regional export performance according to our results except in the models with importer and exporter fixed effects and pair fixed effects. These results provide evidence supporting the importance of road, highway, and railroad stocks for regional export performance with an estimated elasticity of approximately $0.38 \%$. This finding is not very far from the estimated infrastructure elasticity of trade of about $0.42 \%$ for a developing economy estimated in the meta-analytic study presented in Chapter 3. Our estimations suggest that for Turkey, land infrastructure is the type of infrastructure that has the most impact on regional exports, which is another result that is consistent with the above mentioned meta-analytic study. This finding may also be related to the fact that Turkey is adjacent to a trade block such as the EU to which it is connected mainly through land routes. Therefore, regions within Turkey could be heavily reliant on land infrastructure regarding their trade with the EU.

Our estimations present inconclusive results considering the impact of communication infrastructure, represented by DSL per capita. Contrary to expectations, we observe a a negative sign on the elasticities obtained from the OLS model with time dummies, OLS model with multilateral resistance terms, and the regression results with importer fixed effects while models 2 and 3 in Table 4.4 yield positive but insignificant results.

Finally, distance between trading pairs, as expected, is always found to impact on exports negatively and significantly. This result is consistent with the common findings in the trade literature such as those by Limao and Venables (2001) who find a positive effect of distance on trade costs and a negative impact on trade flows, Carrère and Schiff (2005) who observe that for a majority of countries the impact of distance on trade has become increasingly important over time, Berthelon and Freund (2008) who attribute the increasing effect of distance on industrial distance sensitivities, and the meta-analytic studies of Linders (2005) and Disdier and Head (2008), among others. 


\section{Geography}

Common open border positively and significantly affects exports according to most of our results from the models estimated by OLS, OLS with time dummies, and OLS with multilateral resistance terms. This variable is dropped from the model in the importer-exporter and pair fixed effects estimations as it is bilaterally constant through our sample period. Results suggest that adjacency of a region and a country significantly and positively affects regional exports and that it is a relevant control variable. There is also some evidence based on our results from the OLS models with and without year dummies that if a partner country is landlocked, regional exports are negatively affected. However, this effect is no longer observable if multilateral resistances or importer fixed effects are taken into account in column 3 of Table 4.3 and column 1 of Table 4.4 respectively.

\section{Regional demographic characteristics and other core grav- ity variables}

Coefficients of regional population are positive and significant in all our models, suggesting that agglomeration economies export more. On the other hand, while destination country population yields positive estimates, out of these coefficients, those estimated using importer, importer and exporter, and pair fixed effects respectively (in the columns of Table 4.4) are not significant.

The remaining variables are other commonly used factors in trade studies that stem from the theoretical gravity model. We observe the expected positive and significant coefficients for importer country GVA, emphasizing on the role of the destination demand. On the other hand, some evidence is also observed for the positive effect of the origin GVA.

As expected, the larger the value of the Turkish lira in the currency of the trading partner, the less are the export flows. On the other hand, 
if a country enters into a EU customs union agreement with Turkey, regional exports are positively impacted according to the majority of our results. Finally, language similarity exhibits the expected positive impact.

TABLE 4.3

ESTIMATION RESULTS FOR EQUATION (4.12)

\begin{tabular}{|c|c|c|c|}
\hline & $\begin{array}{c}(1) \\
\text { OLS }\end{array}$ & $\begin{array}{c}(2) \\
\text { OLS }\end{array}$ & $\begin{array}{c}(3) \\
\text { MRT }\end{array}$ \\
\hline In Regional (exporter) $G V A_{i t}$ & $\begin{array}{c}0.442^{* * *} \\
(0.0969)\end{array}$ & $\begin{array}{c}0.337^{* * *} \\
(0.117)\end{array}$ & $\begin{array}{c}0.377^{* * *} \\
(0.128)\end{array}$ \\
\hline ln Country (importer) $G V A_{j t}$ & $\begin{array}{c}0.311^{* * *} \\
(0.0166)\end{array}$ & $\begin{array}{c}0.309^{* * *} \\
(0.0166)\end{array}$ & $\begin{array}{c}0.343^{* * *} \\
(0.0172)\end{array}$ \\
\hline $\ln (\text { Distance })_{i j}$ & $\begin{array}{c}-0.777^{* * *} \\
(0.0326)\end{array}$ & $\begin{array}{c}-0.779^{* * *} \\
(0.0327)\end{array}$ & \\
\hline $\ln (\text { Exchange rate })_{j t}$ & $\begin{array}{c}-0.0659^{* * *} \\
(0.00872)\end{array}$ & $\begin{array}{c}-0.0663^{* * *} \\
(0.00870)\end{array}$ & $\begin{array}{c}-0.0807^{* * *} \\
(0.00963)\end{array}$ \\
\hline EU customs union $n_{i j, t}$ & $\begin{array}{c}0.319^{* * *} \\
(0.0900)\end{array}$ & $\begin{array}{c}0.314^{* * *} \\
(0.0906)\end{array}$ & $\begin{array}{c}0.792^{* * *} \\
(0.0872)\end{array}$ \\
\hline Similar language $_{i j}$ & $\begin{array}{c}1.153^{* * *} \\
(0.144)\end{array}$ & $\begin{array}{c}1.152^{* * *} \\
(0.144)\end{array}$ & \\
\hline ln (Land transport infrastructure) it & $\begin{array}{c}0.406^{* * *} \\
(0.151)\end{array}$ & $\begin{array}{c}0.390^{* * *} \\
(0.151)\end{array}$ & $\begin{array}{c}0.382^{* *} \\
(0.161)\end{array}$ \\
\hline $\ln (D S L \text { per capita })_{i t}$ & $\begin{array}{c}0.220 \\
(0.394)\end{array}$ & $\begin{array}{c}-1.467^{* * *} \\
(0.521)\end{array}$ & $\begin{array}{c}-1.394^{* *} \\
(0.553)\end{array}$ \\
\hline $\ln (\text { Air capacity })_{i t}$ & $\begin{array}{c}0.141^{* * *} \\
(0.0173)\end{array}$ & $\begin{array}{c}0.141^{* * *} \\
(0.0173)\end{array}$ & $\begin{array}{c}0.137^{* * *} \\
(0.0189)\end{array}$ \\
\hline $\ln \left(\right.$ Public port capacity) ${ }_{i}$ & $\begin{array}{c}-0.145^{* * *} \\
(0.0290)\end{array}$ & $\begin{array}{c}-0.147^{* * *} \\
(0.0291)\end{array}$ & $\begin{array}{c}-0.152^{* * *} \\
(0.0324)\end{array}$ \\
\hline Number of private ports $s_{i}$ & $\begin{array}{c}0.0427^{* * *} \\
(0.00577)\end{array}$ & $\begin{array}{c}0.0453^{* * *} \\
(0.00596)\end{array}$ & $\begin{array}{c}0.0453^{* * *} \\
(0.00680)\end{array}$ \\
\hline $\ln (\text { Avg. min. distance to airports })_{i}$ & $\begin{array}{l}-0.0107 \\
(0.0358)\end{array}$ & $\begin{array}{l}-0.0580 \\
(0.0412)\end{array}$ & $\begin{array}{l}-0.0585 \\
(0.0455)\end{array}$ \\
\hline
\end{tabular}


TABLE 4.3

ESTIMATION RESUlTS FOR EQUATION (4.12) (CONT'D)

\begin{tabular}{lccc}
\hline \hline & $(1)$ & $(2)$ & $(3)$ \\
& OLS & OLS & MRT \\
\hline In (Avg. min. dist. to major ports) ${ }_{i}$ & $-0.325^{* * *}$ & $-0.326^{* * *}$ & $-0.339^{* * *}$ \\
& $(0.0540)$ & $(0.0542)$ & $(0.0602)$ \\
ln Regional (exporter) Population $n_{i t}$ & $0.427^{* * *}$ & $0.491^{* * *}$ & $0.445^{* * *}$ \\
& $(0.111)$ & $(0.123)$ & $(0.133)$ \\
ln Country (importer) Population $_{j t}$ & $0.0733^{* * *}$ & $0.0742^{* * *}$ & $0.0944^{* * *}$ \\
& $(0.0162)$ & $(0.0161)$ & $(0.0172)$ \\
Landlocked importer ${ }_{j}$ & $-0.484^{* * *}$ & $-0.486^{* * *}$ & 0.0765 \\
& $(0.0534)$ & $(0.0533)$ & $(0.0539)$ \\
Common open border ${ }_{i j}$ & $0.870^{* * *}$ & $0.867^{* * *}$ & $2.132^{* * *}$ \\
& $(0.162)$ & $(0.163)$ & $(0.154)$ \\
ln (MRT corrected distance) $)_{i j}$ & & & $-1.360^{* * *}$ \\
& & & $(0.204)$ \\
Constant & & & $-22.36^{* * *}$ \\
& $-4.634^{* * *}$ & $-3.491^{* * *}$ & $(2.150)$ \\
\hline Observations & $(1.059)$ & $(1.209)$ & 42120 \\
Number of pairs & 42120 & 42120 & 4680 \\
Year Dummies & 4680 & 4680 & Yes \\
Standard errors clustered by & No & Yes & 0.422 \\
\hline Standared & Pair & Pair &
\end{tabular}

Standard errors in parentheses

${ }^{*} p<0.10,{ }^{* *} p<0.05,{ }^{* * *} p<0.01$ 
AdDitional ESTIMATION RESUlts FOR EQUATION (4.12)

\begin{tabular}{|c|c|c|c|}
\hline & $\begin{array}{c}(1) \\
\text { Importer } \mathrm{FE}\end{array}$ & $\begin{array}{c}(2) \\
\text { Imp-exp FE }\end{array}$ & $\begin{array}{c}(3) \\
\text { Pair FE }\end{array}$ \\
\hline In Regional (exporter) $G V A_{i t}$ & $\begin{array}{c}0.309^{* * *} \\
(0.105)\end{array}$ & $\begin{array}{l}-0.103 \\
(0.164)\end{array}$ & $\begin{array}{l}-0.103 \\
(0.164)\end{array}$ \\
\hline In Country (importer) $G V A_{j t}$ & $\begin{array}{l}0.701^{* * *} \\
(0.0900)\end{array}$ & $\begin{array}{l}0.701^{* * *} \\
(0.0898)\end{array}$ & $\begin{array}{l}0.701^{* * *} \\
(0.0895)\end{array}$ \\
\hline $\ln (\text { Distance })_{i j}$ & $\begin{array}{c}-1.327^{* * *} \\
(0.164)\end{array}$ & $\begin{array}{c}-1.360^{* * *} \\
(0.161)\end{array}$ & \\
\hline $\ln (\text { Exchange rate })_{j t}$ & $\begin{array}{c}-0.250^{* * *} \\
(0.0674)\end{array}$ & $\begin{array}{c}-0.250^{* * *} \\
(0.0671)\end{array}$ & $\begin{array}{c}-0.250^{* * *} \\
(0.0669)\end{array}$ \\
\hline EU customs union $n_{i j, t}$ & $\begin{array}{l}0.315^{* * *} \\
(0.0690)\end{array}$ & $\begin{array}{c}0.315^{* * *} \\
(0.0688)\end{array}$ & $\begin{array}{c}0.315^{* * *} \\
(0.0686)\end{array}$ \\
\hline Similar language $_{i j}$ & $\begin{array}{l}0.905^{*} \\
(0.465)\end{array}$ & & \\
\hline ln (Land transport infrastructure) ${ }_{i t}$ & $\begin{array}{c}0.395^{* * *} \\
(0.137)\end{array}$ & $\begin{array}{l}-0.378 \\
(0.270)\end{array}$ & $\begin{array}{l}-0.378 \\
(0.269)\end{array}$ \\
\hline $\ln (D S L \text { per capita })_{i}$ & $\begin{array}{c}-1.519^{* * *} \\
(0.461)\end{array}$ & $\begin{array}{c}0.408 \\
(0.360)\end{array}$ & $\begin{array}{c}0.408 \\
(0.359)\end{array}$ \\
\hline $\ln (\text { Air capacity })_{i t}$ & $\begin{array}{c}0.143^{* * *} \\
(0.0149)\end{array}$ & & \\
\hline $\ln (\text { Public port capacity })_{i}$ & $\begin{array}{c}-0.143^{* * *} \\
(0.0249)\end{array}$ & & \\
\hline Number of private ports $s_{i}$ & $\begin{array}{c}0.0454^{* * *} \\
(0.00535)\end{array}$ & & \\
\hline $\ln (\text { Avg. min. distance to airports })_{i}$ & $\begin{array}{l}-0.0576 \\
(0.0369)\end{array}$ & & \\
\hline $\ln (\text { Avg. min. dist. to major ports })_{i}$ & $\begin{array}{c}-0.316^{* * *} \\
(0.0483)\end{array}$ & & \\
\hline In Regional (exporter) Population $i t$ & $\begin{array}{c}0.524^{* * *} \\
(0.111)\end{array}$ & $\begin{array}{c}0.992^{* * *} \\
(0.172)\end{array}$ & $\begin{array}{c}0.992^{* * *} \\
(0.171)\end{array}$ \\
\hline
\end{tabular}


TABLE 4.4

Additional estimation Results For EQuation (4.12) (CONT'D)

\begin{tabular}{lccc}
\hline \hline & $(1)$ & $(2)$ & $(3)$ \\
& Importer FE & Imp-exp FE & Pair FE \\
\hline ln Country (importer) Population $j$ t & 0.0163 & 0.0163 & 0.0163 \\
& $(0.225)$ & $(0.223)$ & $(0.223)$ \\
& & & \\
Landlocked importer $j$ & -0.461 & & \\
& $(0.431)$ & & \\
Common open border $i j$ & -0.630 & & \\
& $(0.479)$ & & $-7.180^{* * *}$ \\
Constant & -2.043 & $6.759^{* *}$ & $(2.414)$ \\
& $(2.172)$ & $(3.159)$ & 42120 \\
& 42120 & 42120 & 4680 \\
Observations & 4680 & 4680 & Yes \\
Number of pairs & Yes & Yes & Not clustered \\
Year Dummies & Pair & Pair & 0.2556 \\
Standard errors clustered by & 0.534 & 0.547 & 141.005 \\
R-squared & & & \\
Hausman test statistic & & & \\
\hline
\end{tabular}

Standard errors in parentheses

${ }^{*} p<0.10,{ }^{* *} p<0.05,{ }^{* * *} p<0.01$

Importer fixed effects model uses a dummy variable for each importer. ImporterExporter fixed effects includes dummies for each importer, and dummies for each exporter separately, pair fixed effects includes a dummy for each trading pair.

\subsection{Concluding Remarks}

The previous chapter (Chapter 3) presented a meta-analysis on the infrastructure elasticities of trade obtained from 36 previous studies. According to the results, estimations that used a land transport infrastructure variable found significantly higher infrastructure elasticities 
of trade relative to estimations using other infrastructure types. The results in Tables 4.3 and 4.4 are in line with this observation except for the models using importer-exporter and pair fixed effects, which yield insignificant elasticities of land transport infrastructure. Chapter 3 also suggests that estimations focusing on maritime or air transport infrastructure find a significantly higher estimate of the impact of importer's infrastructure. While in this chapter, the focus is on only the exporter's infrastructure, results regarding these two types of infrastructure reinforce the conclusion that they play an important role in trade facilitation, except for public port capacity, as seen in both Tables 4.3 and 4.4. Therefore, the continuation of public investments in these types of public infrastructure are recommended. Results on ports yield a different story; as the number of private ports exhibit a positive impact on exports, public port capacity does not. This may point to certain efficiency differences between publicly and privately managed ports. However, access to public ports measured in terms of distance still has a positive effect on regional exports. Thus, the results of this chapter also show that the location of point infrastructure is important for regions regardless of a public-private distinction. As a result, the findings of this study underline the roles of land, port, and airport transport infrastructures in the exports of Turkey, and also the importance of the spatial distribution of point infrastructures as exit nodes for exports. 


\section{A Appendix}

\section{A.1 Additional estimation results}

Column 1 in Table 4.A.1 presents the results of the panel estimation with random effects. While most of the coefficients are similar to those from the previous results, the earlier reported Hausman test suggests that the pair fixed effects results reported in column 3 of Table 4.4 should be preferred. On the other hand, the pair fixed effects regression drops many key variables due to them being constant, causing loss of valuable information.

Sample selection bias can potentially arise in trade research as only those countries that engage in trade are used in the regression analyses (Helpman et al., 2008). We conduct a maximum likelihood estimation of a Heckman sample selection model for our empirical specification. ${ }^{18}$ Column 2 of Table 4.A.1 suggests that sample selection is not an issue in our analyses, as the inverse Mill's ratio is insignificant. The selection variable is Open common border as mentioned in section 5.5. The neighbors of Turkey are Georgia, Armenia, the Nakhchivan enclave of Azerbaijan ${ }^{19}$, Iran, Syria, Iraq, Bulgaria, and Greece. Due to political frictions, the border between Armenia and Turkey is closed, hence the name Common open border for this variable. On the other hand, Armenia is dropped from the sample due to too little trade in the procedure detailed in section 5.4. Therefore the term "open" in this variable is no longer relevant in the analysis. The most important difference of the results from the Heckman estimation compared to our other results is that communication infrastructure has the expected positive and significant coefficient. On the other hand, land transport infrastructure has an insignificant coefficient.

\footnotetext{
${ }^{18}$ Puhani (2000) suggests that the full-information maximum likelihood estimator can be preferred over the two-step method of Heckman (1979) if collinearity problems are not present.

${ }^{19}$ We do not consider Azerbaijan as a neighbor of Turkey as the enclave represents only a very small part of its economy.
} 
PAir RE AND HECKMAN ESTIMATION RESUlts FOR EQUATION (4.12)

\begin{tabular}{|c|c|c|c|}
\hline & \multirow{3}{*}{$\begin{array}{c}(1) \\
\text { Pair RE }\end{array}$} & \multicolumn{2}{|c|}{$(2)$} \\
\hline & & \multicolumn{2}{|c|}{ Heckman MRT } \\
\hline & & Outcome & Selection \\
\hline \multirow[t]{2}{*}{ In Regional (Exporter) GVA } & 0.0508 & $1.185^{* * *}$ & $0.775^{* * *}$ \\
\hline & $(0.0989)$ & $(0.200)$ & $(0.113)$ \\
\hline \multirow[t]{2}{*}{ ln Country (importer) $G V A_{j t}$} & $0.325^{* * *}$ & $0.452^{* * *}$ & $0.295^{* * *}$ \\
\hline & $(0.0163)$ & $(0.0307)$ & $(0.0138)$ \\
\hline \multirow[t]{2}{*}{$\ln (\text { distance })_{i j}$} & $-0.770^{* * *}$ & & \\
\hline & $(0.0318)$ & & \\
\hline \multirow[t]{2}{*}{ ln (Exchange rate) $)_{j t}$} & $-0.0658^{* * *}$ & $-0.139^{* * *}$ & -0.00769 \\
\hline & $(0.00866)$ & $(0.0162)$ & $(0.00764)$ \\
\hline \multirow[t]{2}{*}{$E U$ customs union $_{i j, t}$} & $0.319^{* * *}$ & $0.940^{* * *}$ & $0.577^{* * *}$ \\
\hline & $(0.0575)$ & $(0.0899)$ & $(0.0589)$ \\
\hline \multirow[t]{2}{*}{ Similar language $_{i j}$} & $1.156^{* * *}$ & & \\
\hline & $(0.144)$ & & \\
\hline \multirow[t]{2}{*}{ ln (land transport infrastructure) it } & 0.133 & 0.232 & 0.0627 \\
\hline & $(0.138)$ & $(0.255)$ & $(0.136)$ \\
\hline \multirow[t]{2}{*}{$\ln (D S L \text { per capita })_{i}$} & 0.295 & 0.691 & $1.543^{* * *}$ \\
\hline & $(0.346)$ & $(0.816)$ & $(0.431)$ \\
\hline \multirow[t]{2}{*}{$\ln (\text { Air capacity })_{i t}$} & $0.110^{* * *}$ & $0.114^{* * *}$ & -0.0182 \\
\hline & $(0.0173)$ & $(0.0307)$ & $(0.0147)$ \\
\hline \multirow[t]{2}{*}{$\ln \left(\right.$ Public port capacity) ${ }_{i}$} & $-0.195^{* * *}$ & $-0.287^{* * *}$ & $-0.208^{* * *}$ \\
\hline & $(0.0289)$ & $(0.0486)$ & $(0.0229)$ \\
\hline \multirow[t]{2}{*}{ Number of private ports $s_{i}$} & $0.0530^{* * *}$ & $0.0518^{* * *}$ & -0.00124 \\
\hline & $(0.00573)$ & $(0.00886)$ & $(0.00425)$ \\
\hline \multirow[t]{2}{*}{ ln $(\text { Avg. min. distance to airports })_{i}$} & $-0.111^{* * *}$ & $0.197^{* * *}$ & -0.00594 \\
\hline & $(0.0376)$ & $(0.0604)$ & $(0.0396)$ \\
\hline \multirow[t]{2}{*}{$\ln (\text { Avg. min. dist. to major ports })_{i}$} & $-0.426^{* * *}$ & $-0.564^{* * *}$ & $-0.608^{* * *}$ \\
\hline & $(0.0533)$ & $(0.0891)$ & $(0.0423)$ \\
\hline
\end{tabular}


TABle 4.A. 1

PAir RE AND Heckman ESTIMATion RESUlts FOR EQUATion (4.12) (CONT'D)

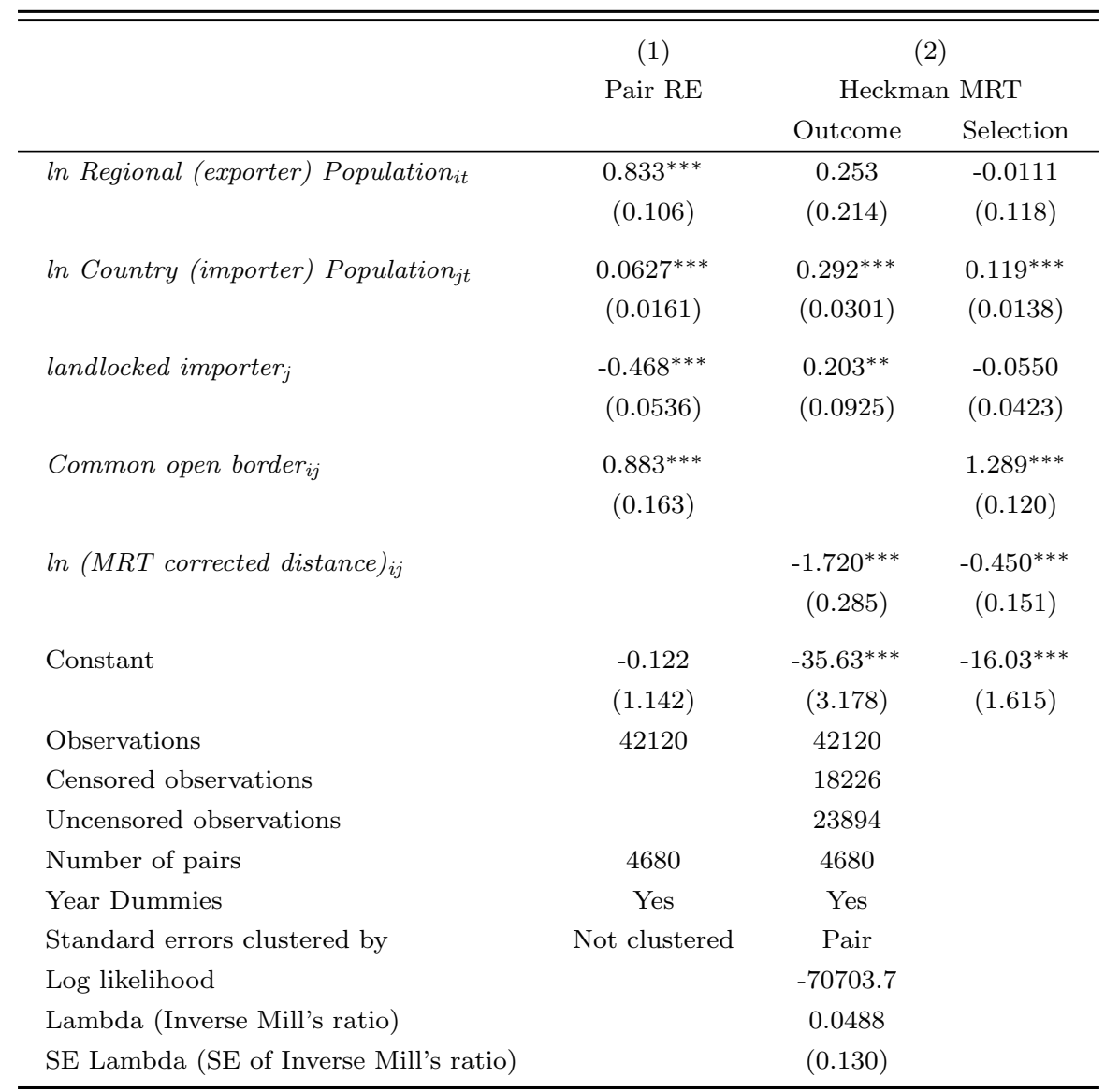

Standard errors in parentheses

${ }^{*} p<0.10,{ }^{* *} p<0.05,{ }^{* * *} p<0.01$ 


\section{A.2 Conversion to constant prices}

The GVA and public investment (PI) data has been compiled in terms of 1998 national currency. According to the T.R. Ministry of Development, the external currency deflator to 2005 for 1998 (national currency) is $0.1415 .^{20}$ The regional GVA and PI figures were divided by this deflator to convert them to 2005 prices. The USD conversion rate specified in the same source was $1 \mathrm{USD}=1.67 \mathrm{TL}$ for 2005 . Therefore the GVA and PI values in 2005 constant national currency were divided by 1.67 and converted to dollars.

\section{A.3 Land infrastructure index construction}

The weights presented in footnote (4) were used to create the land infrastructure index using road length, highway length, and railroad length per $1000 \mathrm{sqm}$ as follows: the total share of road and railroad in exports in 2009 was $0.417+0.09=0.507$ (so about half of total exports in were made through these types of infrastructure).

$0.417 \div 0.507=0.82249$ is the weight of roads in land infrastructure and $0.09 \div 0.507=0.17751$ is the weight of railroads in land infrastructure. The index "land" is calculated as follows:

land $=0.822485207100592 \times[\ln ($ road per area $)+\ln ($ highway per area $)]$ $+0.177514792899408 \times[\ln ($ railroad per area $)]$

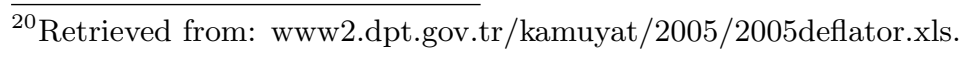




\section{A.4 Exchange rates}

Exchange rates to USD for each partner country are from Penn World Table 7.1 (Heston et al., 2012). ${ }^{21}$ Each country's exchange rate to USD was divided by Turkey's exchange rate to USD in a given year so that all observations express the amount of the corresponding foreign currency one Turkish lira can buy within a specific year.

\section{A.5 DSL data imputation}

The observations for 2002 and 2005 for each region is missing for the DSL (or ADSL) data. ${ }^{22}$ The missing values were imputed using the regional public investments in transportation and communication (TPI) by predicting the missing values for each region $i$ using the coefficients from the OLS estimation of the below equation:

$\ln (a d s l)_{t}=a+\beta(T P I)_{t-1}+\epsilon_{t}$

\footnotetext{
${ }^{21}$ The rates for China are from "version 2" of this table.

${ }^{22}$ For 2003, the figure is reported as "DSL" where as for the rest of the years they are reported as "ADSL."
} 
TABle 4.A.2

REGION CODES AND NAMES

TR10: Istanbul

TR21: Tekirdag, Edirne, Kirklareli

TR22: Balikesir, Canakkale

TR31: Izmir

TR32: Aydin, Denizli, Mugla

TR33: Manisa, Afyon, Kutahya, Usak

TR41: Bursa, Eskisehir, Bilecik

TR42: Kocaeli, Sakarya, Duzce, Bilecik

TR51: Ankara

TR52: Konya, Karaman

TR61: Antalya, Isparta, Burdur

TR62: Adana, Mersin

TR63: Hatay, Kahramanmaras, Osmaniye

TR71: Kirikkale, Aksaray, Nigde,

TR72: Kayseri, Sivas, Yozgat

TR81: Zonguldak, Karabuk, Bartin

TR82: Kastamonu, Cankiri, Sinop

TR83: Samsun, Tokat, Corum, Amasya

TR90: Trabzon, Ordu, Giresun, Rize

TRA1: Erzurum, Erzincan, Bayburt

TRA2: Agri, Kars, Igdir, Ardahan

TRB1: Malatya, Elazig, Bingol, Tunceli

TRB2: Van, Mus, Bitlis, Hakkari

TRC1: Gaziantep, Adiyaman, Kilis

TRC2: Sanliurfa, Diyarbakir

TRC3: Mardin, Batman, Sirnak, Siirt 


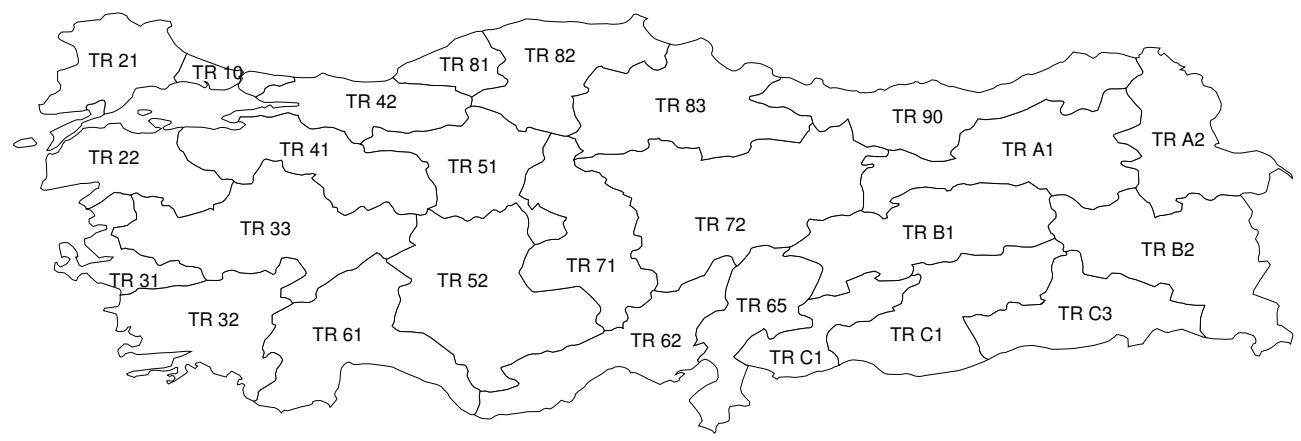

Figure 4.A.1. NUTS-2 LEVEL REgIONAL MAP OF TURKEy. 


\section{CHAPTER 5}

\section{Public Investment and Regional Politics: The Case of Turkey ${ }^{1}$}

\section{$5.1 \quad$ Introduction}

The contributions of public infrastructure to economic growth have been researched and advocated since the early 1900's. Especially transportation and communication infrastructure has drawn much attention. It is commonly recognized as a stylized fact that transportation infrastructure forms and strengthens the links between economic areas, facilitates the mobility of goods, input factors, human capital, and creates positive externalities to firms and industries (Lakshmanan et al., 2001; Persky et al., 2013). Communications infrastructure on the other hand, is argued to play an important role in the "transportation" of information: it reduces the "information gap" between markets (Carey, 2008), and shapes the economic geography by impacting on financial services and capital flows (Dokmeci and Berkoz, 1996) while still being physically attached to specific locations (Castells et al., 2007). These arguments imply that transportation and communication infrastructures are especially relevant in a spatial context.

The allocation of these types of infrastructure across sub-national regions

\footnotetext{
${ }^{1}$ This chapter is the result of a joint work with Denis de Crombrugghe and Joan Muysken. This study is also available online as a UNU-MERIT working paper with serial number 2014-020, co-authored by Denis de Crombrugghe and Joan Muysken.
} 
within a national economy has been a matter of debate for economists and politicians across the world. This allocation process requires decision makers to take into account regional and national needs in conjunction with region-specific characteristics. As a result, this process can be subject to many factors such as geographical, locational, demographic, economic, and political attributes of the investment receiving regions. Moreover, the motives regarding welfare, equality, and efficiency may differ between economies and decision-makers, presenting heterogeneity in national goals within the context of regional policy.

While for many developed countries, the spatial allocation of government services and/or infrastructure is commonly researched, this is not always the case for developing economies. An example is Turkey, where the regional allocation of infrastructure has often been part of political debate, but not a subject of academic research. This may have been due to the previous unavailability of a sufficiently long time-series data which was a major limitation that we tackled in this study: a meticulous data collection process from fragmented resources allowed us to attain a panel data set that has a time dimension of 13 years. ${ }^{2}$ As a result, we have been able to conduct our analyses using a time-series cross-sectional data set from the twenty-six statistical regions of Turkey through the years 1999-2011, and contribute new evidence to the literature regarding a previously non-examined country.

Another novelty that this research presents is the consideration that investments in the separate infrastructure categories may not be decided independently from one another. In this regard, we assume that the allocation decisions of all categories of public capital are made jointly (i.e. they are all subject to the same resource constraint). Thus, we do not treat investments in transportation and communication infrastructure as being independent from investments in other types of public capital. This assumption and its relevance to our research is discussed further in Section 5.3.

\footnotetext{
$\overline{{ }^{2} \text { Our models use a maximum of } 12}$ years of observations due to the presence of lagged variables in the estimations.
} 
The rest of this study is structured as follows. Section 5.2 provides a review of the past literature and elaborates on the motivation and contribution of this paper, followed by an overview of the trends in regional infrastructure investments in Turkey and the regional governance structure of the country. Section 5.3 elaborates on the theoretical framework and on how it leads to our empirical analysis. The data collected is described in Section 5.4. Section 5.5 presents the empirical results and elaborates on their implications, followed by the concluding discussion in Section 5.6.

\subsection{Research Motivation and Contribution}

\subsubsection{Public capital and regional goals}

Our point of departure is the stylized fact that transportation and communication public infrastructure provides a positive contribution to an economy through various channels. For instance, positive impact of public capital investments on economic growth has been observed in studies by Aschauer (1989a,b), Munnell and Cook (1990); Munnell (1990), Garcia-Mila and McGuire (1992), Aschauer (2000) for the United States, León-González and Montolio (2004) for Spanish provinces, Bom and Ligthart (2008) through meta-analysis, Hamalainen and Malinen (2011) for Finnish regions, among others. ${ }^{3}$

Specifically, transportation public capital has been a highly researched infrastructure category in relation to economic growth; research is done by Stephan (2001) for German and French regions, Cadot et al. (1999) for French regions, Berechman et al. (2006) for the United States, Montolio and Sole-Olle (2009) and Cantos et al. (2005) for Spanish provinces have found a positive relationship between growth and this type of capital. ${ }^{4}$

\footnotetext{
${ }^{3}$ See Romp and De Haan (2007) for a comprehensive survey of the recent literature focusing on this relationship.

${ }^{4}$ Bhatta and Drennan (2003) provide an extensive survey of the literature focusing on the relationship between public investment in transportation and economic
} 
In addition, transportation infrastructure, together with communication infrastructure, has been found to influence trade performance positively as shown by Bougheas et al. (1999) for nine core EU and Scandinavian countries, Limao and Venables (2001) for 103 World Countries, Martínez-Zarzoso and Nowak-Lehmann (2003) for a sample of 20 EU and Mercosur countries and Chile, Longo and Sekkat (2004) for intra-African trade, $\mathrm{Wu}$ (2007) for Chinese regions, and through meta-analysis in Chapter 3. It has also been shown that public investments can benefit an economy through other channels as well; Altunc and Senturk (2010) find that infrastructural public investments have stimulated private investments in Turkey between 1980 and 2009, Holtz-Eakin and Lovely (1996) observe a positive impact of public capital on the expansion of the manufacturing sector in the United States, and Ding et al. (2008) find that telecommunications infrastructure has played an important role in regional per-capita income convergence in China during the period 1986-2002.

Turning to the determinants of public investments, population effects have been hypothesized to be of relevance since the early $20^{\text {th }}$ century. In a relatively early study, Hirsch (1959) observed, contrary to previous findings in the literature suggesting population size as an important factor, that for a wide range of urban service expenditures, population size does not matter but geographical size does. On the other hand, Hansen (1965) reported that for a sample of Belgian communities, the concentration of population is associated with higher public investment, while in a country-level panel study, Randolph et al. (1999) find that factors such as the level of development, urbanization, population density, and labor force participation have strong implications on per capita spending on infrastructure in transportation and communication.

Together with population-related factors, economic variables have naturally also drawn attention in the literature. In this context, there has been a surging interest in the equity-efficiency preference in the allocation of public infrastructure as defined in Yamano and Ohkawara (2000) and Castells and Sole-Olle (2005). The equity-efficiency trade-off

development. 
is the choice between investing in spatial sections of an economy with relatively higher productivity for attaining higher national efficiency, and investing into those that are lagging for achieving regional equity. In this regard, Nijkamp states that

"In the light of economies of scale and scope, there may be a tendency to invest heavily in central areas, as here in general the expected benefits per unit of investment and per capita are the highest. Of course, this may be at odds with spatial equity targets, and therefore it may not be so easy to find a proper balance between the goals of efficiency and equity in a regional competition context." Nijkamp (2000, p.89).

In relation to regional equity goals, the inter-regional infrastructure investment can also be viewed as a redistributive policy as proposed by Sole-Olle (2011): money is re-allocated between regions through the regional investment of the funds which, in turn, are collected through taxes paid by regions. ${ }^{5}$ Related empirical results vary depending on the economy and the time period in question. Mizutani and Tanaka (2008) for Japan in 1975-1990, and Castells and Sole-Olle (2005) for Spain in 1987-1996 observe that relative to the national governments, sub-national units value efficiency more. On the other hand, Yamano and Ohkawara (2000) find that the Japanese central government has adopted a policy of equity regarding the allocation of public investments between 1970 and 1994 in their study on forty-seven prefectures.

\subsubsection{Political influences and investment allocation}

With respect to political factors in relation to the spatial allocation of public capital, Crain and Oakley (1995, p.15) state that "...public capital decisions are not made in political vacuum" and in their study

\footnotetext{
${ }^{5}$ The author distinguishes infrastructure investment redistribution motives into two categories: tactical and programmatic. In tactical redistribution few regions receive the benefits, and costs are shared by all regions. On the other hand, programmatic redistribution specifically aims to withdraw resources from certain regions and redistributes them to others (Sole-Olle, 2011).
} 
on US states, find that various political and institutional conditions influence public capital decisions. Similarly, in a study on the regions of France, which has a very similar regional governance structure to the country of focus in this study, ${ }^{6}$ Cadot et al. (1999) observe that "influence activities" represented by a political variable have important implications on the regional allocation of transportation infrastructure. Regarding the same type of infrastructure, Painter and Bae (2001) point out to a significant influence of political factors along with demographic and economic determinants in their study on US states. Similar effects of politics on the spatial allocation of public investment for various specific cases are demonstrated by Kemmerling and Bodenstein (2006), Busemeyer (2007), and Kemmerling and Stephan (2008), and specifically in the context of political affiliation of the investment receiving units to the decision makers, by Costa-I-Font et al. (2003), Castells and Sole-Olle (2005), Joanis (2011), Sole-Olle (2011), and Zheng et al. (2013).

Public investments can also be seen by decision makers as a way to increase their election probabilities (Nijkamp, 2000). Moreover, the political structure of regional administration can have various implications on the investment decision process. For example, in a decentralized economy where regions make their own investment decisions, Yu et al. (2011) find that public investments in neighboring regions play an important role. Therefore, spatial dependence may exist if regions behave based on each others' investment choices.

The possible existence of strong political influences in the regional allocation of public investments is an ongoing debate in Turkey. ${ }^{7}$ While evidence for such influences has been shown in a case-specific way in several previous studies done on various other countries, the significance and magnitude of these influences can seldom be generalized to other

\footnotetext{
${ }^{6}$ Turkey has been taking the French regional governance system as a model (Gokyurt, 2010).

${ }^{7}$ Examples of claims regarding the existence of this political influence can be seen in articles recently published in major national newspapers such as at http://www.radikal.com.tr/yazarlar/koray_caliskan/tayyip_erdogandan_izmire_ buyuk_ceza-1180596 and at http://www.milliyet.com.tr/izmir-i-akp-ye-oy-vermedigiicin-/gundem/detay/1856652/default.htm.
} 
cases. This is because results highly depend on the political and institutional cultures and habits in the specific country of focus, and the specific industry considered (for instance, the level of political influences in the allocation in health services does not need to correspond to the political influence levels in the allocation of transportation services). Therefore, each economy and each industry within an economy may require special attention. Moreover, the findings may differ between time periods as well especially if these periods correspond to certain political climates. In this regard, the public investment allocation in the transportation and communication sector of Turkey during the period 1999-2011 requires special attention as this period is mostly characterized by a single-party government and a surge in investments in transportation and communication infrastructure.

Together with providing novel results on the regional allocation of transportation and communication public infrastructure in Turkey, our study also makes a theoretical contribution to the general approach of looking at the question of industry-specific regional public investment allocation by considering the other public investments a region receives; it is often the case that when looking at the allocation of a specific type of public capital among regions, the investments a region receives in the other categories of public capital are ignored. However, it may well be the case that investments in different sectors may be complements, or substitutes. Moreover, they are subject to the same government budget constraint (i.e. the source of funds from which public investments are made is the same for all categories). This could imply endogeneity in the empirical examination of allocation decisions: the investment categories may be each others' determinants. Aside of a substitution effect between separate public investment categories, a positive dependency (complementarity) could also exist. As a result, in our analyses, we acknowledge and control for this possible dependence among investments made in different public sectors. Further discussion on how this dependency is considered in our estimations are presented in Sections 5.3 and 5.5.

In the literature, the measurement of political effects are diverse. Some examples are presented in Table 5.A.2 which demonstrates the large variety of ways that political affiliations of investment receiving units 
to decision-makers is measured. This variation in measurement choices is mainly due to the diversity of countries in terms of their political structures and regional governance schemes. This leaves us some room to construct our own variable for political affiliation in order to take into account the political influences which is consistent with Turkey's centralized structure of territorial governance. This measurement is discussed in detail in Section 5.3.

\subsubsection{Regional public infrastructure in Turkey}

Since the establishment of the Republic of Turkey, regional policy goals have been shaped by five-year development plans made by the State Planning Organization (SPO) which was redefined as the "Ministry of Development" in 2011. For consistency, we use the label "SPO" for this governing body throughout this study. ${ }^{8}$ Turkey has 26 statistical regions and an area of $783,562 \mathrm{~km}^{2}$. These twenty-six regions are composed of varying numbers of provinces which add-up to a country total of eighty-one. ${ }^{9}$ Along with the existence of local governing bodies and the gradual introduction of Regional Development Agencies (RDA's), the SPO is currently the principal body of decision concerning public investments. ${ }^{10}$ Regarding local governance in Turkey, Lagendijk et al. state that

"....it is important to remember that the current territorial governance structure, based on a division into 81 provinces, primarily serves to carry out basic administrative tasks under central authority." (Lagendijk et al., 2009, p.386)

This mechanism of centralized decision making attracts some criticism. For example, Gokyurt (2010) points out that the approach on the public investment process in Turkey suffers from an over-focus on central

${ }^{8}$ For summaries of the five-year plans undertaken by the SPO see Keskin and Sungur (2010) and Ersoy (2014).

${ }^{9}$ Table 5.A.1 lists the 26 statistical regions and their NUTS 2 level codes that cover all of Turkey's territory.

${ }^{10}$ For a detailed up-to-date explanation of the role of the SPO in regional policy-making see Ertugal and Dobre (2011). 
and sectoral points of view, and argues that the low contribution to public investment decisions by the local level causes inconsistencies between spatial needs and public investment plans. Moreover, the central organization (SPO) is fully under the authority of the government.

In country-aggregate terms, income and public investments have generally had an upward trend in Turkey during the last decade. Figure 5.1 compares the trends in the country gross domestic product (GDP) and transportation and communication public investments (abbreviated as "TPI" in the figure) through the years 1999-2011. An upwards trend for both variables is prominent especially for the period after 2002, which coincides to a post-crisis period and an election of a single-party government.

Figure 5.1

GVA AND PUBLIC INVESTMENTS IN TRANSPORTATION

AND COMMUNICATION, CONSTANT 1998 NATIONAL

CURRENCY (BILlions), TURKEy.

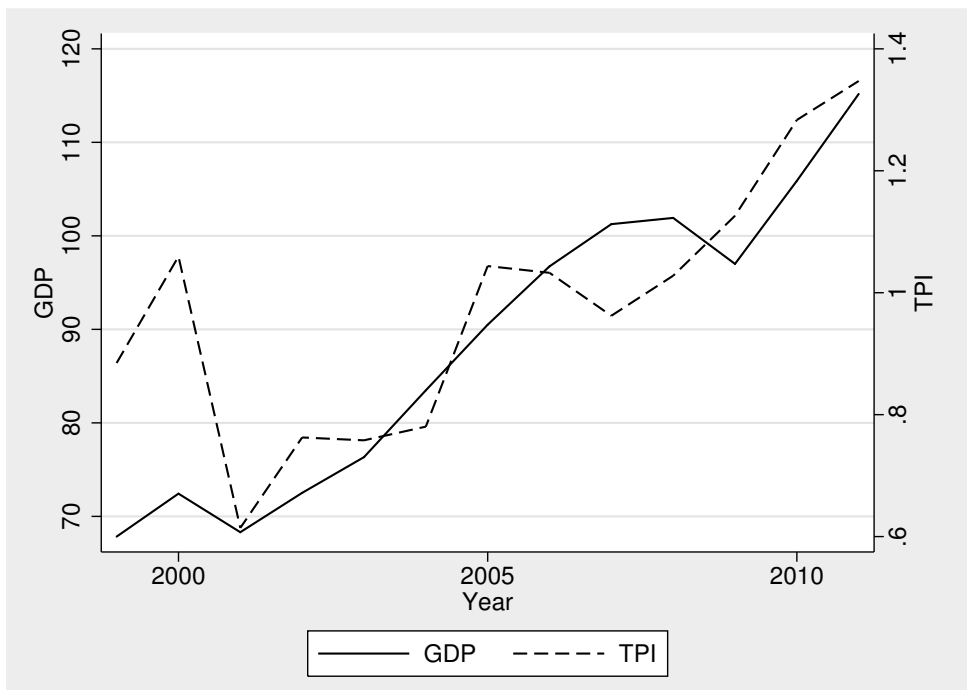




\subsection{Theoretical Framework and Empirical Ap- proach}

To analyze the determinants of the regional allocation of transportation and communication public investments in Turkey, we follow the theoretical framework of Behrman and Craig (1987) as adapted by Castells and Sole-Olle (2005) and Zheng et al. (2013). According to this approach, a central government facing budget and production constraints aims to maximize country welfare by allocating the public investments between regions subject to a trade-off between efficiency and regional equity which is embodied in the linear combination of two variables: output and population (Castells and Sole-Olle, 2005). This approach suggests that if the government is only concerned about regional equity, then regional population is the only characteristic that the government considers in the allocation of public investments. On the other hand, if the only concern of the government is efficiency, then the only determining factor is the regional per-capita output (Castells and Sole-Olle, 2005). ${ }^{11} \mathrm{We}$ represent equity and efficiency in our model according to this approach and denote the population in region $i$ at time $t$ as $N_{i t}$, and the regional Gross Value Added (GVA) per capita as $\frac{Y_{i t}}{N_{i t}}$ where $Y$ is the regional GVA.

The allocation process is also affected by the weights the government places on each region (Castells and Sole-Olle, 2005; Zheng et al., 2013). These weights are determined by a set of regional characteristics. Nonuniform weights across regions can result in an allocation process that is not driven purely from an economic point of view. In other words, a political dimension in the process would be also present aside of the equity-efficiency trade-off. As discussed in Section 5.2, political factors, especially in the form of political affiliation, are frequently considered by researchers as determinants of these weights, and therefore, of public investment allocation.

\footnotetext{
${ }^{11}$ It is assumed that such a government considers that welfare is increased only by increasing the national output and considers that regional equity has no effect on national welfare. Its expectation is that that investing in developed regions will achieve higher increase in national output.
} 
An obvious influence of a regional economy on the decisions of the government is through the individuals that take place in central decisionmaking. In Turkey, every region can send a fixed number of elected members of parliament (MP's) to the national assembly. If the share of the MP's from a given region in the government within the total number of MP's in the parliament from the same region is relatively high, then a positive political bias towards this region can be expected regarding the allocation of transportation and communication infrastructure. ${ }^{12}$ Therefore our measure of political affiliation, $P$, is the share of the MP's a region has in the central government party (or parties) out of all its MP's in the parliament such that $P_{i t}=\frac{\text { No. of } M P_{i t} \text { in the gov't }}{\text { no. of } M P_{i t}}$ where $M P_{i t}$ is the number of MP's from region $i$ at time $t .^{13}$

It is likely that decision makers observe the information on regional political affiliation from the previous year as opposed to having instant access to this knowledge (Castells and Sole-Olle, 2005). Therefore, we include $P_{i t}$ by lagging it one year in our estimations. On the other hand, information regarding the economic and demographic variables can be more readily available, as monthly or quarterly estimates usually exist. Therefore, these variables can be expected to have instant effects on allocation decisions of policy-makers. ${ }^{14}$

As discussed Section 5.2, a potential source of endogeneity can be caused by the fact that public investments in transportation and communication, denoted as $I_{i t}$ in our analysis, may not be independent from the other types of public investments. We recognize a second category of "all other public investments" as opposed to our main focus variable $\left(I_{i t}\right)$. These two categories of investment are both part of the total regional

\footnotetext{
${ }^{12}$ The measurement of all variables including $P_{i t}$ are detailed in Table 5.A.3.

${ }^{13}$ We use the number of MP's instead of a measure that takes regional population into account such as the number of MP's per capita. This is because the number of MP's a region sends to the parliament is decided based on its population size. Therefore, volume effects are already considered.

${ }^{14}$ Even though election polls are common, they are made by private companies and results can exhibit great variation between polling firms. Therefore political tendencies may require some time and consensus to be confirmed, while official information on the economy and the demography can be more readily available to the decision-makers.
} 
public investments. This implies that they are likely to be subject to the same government budget constraint. As a result, their regional allocation decisions are expected to be made jointly. Therefore, the public investments made in all other categories of infrastructure are specifically included as an endogenous variable in our model, denoted as $O_{i, t}$.

Zheng et al. (2013) point out that investment projects may take multiple years, and investments made in a given year can bring further investments in the subsequent periods. ${ }^{15}$ Following this view, we include into our model a lag of the dependent variable, $I_{i, t-1}$ in order to take into account the expectation that investment flows may be correlated across consecutive time periods.

Aside of political factors, there still is a wide range of regional characteristics that need to be taken into account for controlling the earlier discussed regional weights placed by the government on individual regions. We assume that decision makers take into account the regional needs by considering the already existing infrastructure within a region before allocating the infrastructure investments. As a result, we control for the effect of the existing stock of transportation and communication infrastructure in a region by including the variable $G_{i t}$ in lagged form, which is an infrastructure stock index constructed using the first principal components of the natural logarithms of the variables Road density, Hway density, Railway density, Public Piers, Air capacity, and ADSL. ${ }^{16}$ Instead of including all the infrastructure categories in a disaggregated manner in the estimations, we use this combined index in order to keep the consistency with the dependent variable which is itself the combined public investment value in all these infrastructure categories. Thus, $G_{i t-1}$ measures how endowed a region was in terms of general transportation and communication infrastructure in the preceding year. We expect that a central policy-maker who is concerned with regional disparities in terms

\footnotetext{
${ }^{15}$ Unlike the empirical specification of Zheng et al. (2013), our model does not take into account the spatial interdependence of investments. Elhorst (2012) points out to the many econometric problems in the currently available dynamic spatial panel data estimators (ML, QML, IV/GMM, and Bayesian MCMC).

${ }^{16}$ The definitions and measurements of these variables are presented in Table 5.A.3.
} 
of infrastructure endowment would direct future period investment to regions with less current infrastructure stock, which would be observed as a negative coefficient estimate on $G_{i, t-1}$. This expectation is also in line if one considers the capital accumulation relation which would translate to our case as (leaving depreciation aside) $I_{i t}=G_{i t}-G_{i, t-1}$. On the other hand, if the motivation is to enhance the infrastructure in regions with already high levels of infrastructure stock, this variable would yield a positive estimate.

Another regional attribute that is related to infrastructure is pointed out by Glomm and Ravikumar (1994, p.1174) who state that "the contribution of infrastructure to private factor productivity is subject to congestion." This view is supported by Castells and Sole-Olle (2005) who state that the utilization level of transport infrastructure stock has consequences on the services provided by infrastructure. For measuring this congestion effect, Fernald (1999) uses the total miles driven by trucks and automobiles. We use the vehicle stock per capita in a region as an indicator of congestion and denote this variable as $V_{i t} / N_{i t}$ where $V$ represents the total regional vehicle stock.

Based on the above discussion, our core empirical specification takes the following form:

$$
\begin{aligned}
\ln I_{i t} & =c_{0}+\beta_{1} \ln I_{i, t-1}+\beta_{2} \ln O_{i t}+\beta_{3} \ln \left(\frac{Y_{i t}}{N_{i t}}\right)+\beta_{4} \ln N_{i t}+\beta_{5} \ln P_{i, t-1} \\
& +\beta_{6} G_{i, t-1}+\beta_{7} \ln \left(\frac{V_{i t}}{N_{i t}}\right)+c_{i}+c_{t}+e_{i t}
\end{aligned}
$$

where $c_{0}$ is a constant and $e_{i t}$ is the error term. As earlier discussed, the allocation of investments among regions are subject to weights determined by a set of regional characteristics. Those characteristics that are time-invariant are assumed to be partly within $c_{i}$, and those that are region-invariant are assumed to be partly within $c_{t}$. 
Some time-invariant geographical conditions of a region that affect public infrastructure projects can be readily observed. Ramcharan (2009) documents that the transport networks of countries with rougher surfaces are less developed than those with less rough terrain surface. In support of this conclusion, Martincus et al. (2012, p.11), state "roughness imposes severe challenges to development and maintenance of transport networks." Within these lines, we hypothesize that regional hilliness (or roughness) can either discourage investment, or require more costly investment projects and increase regional needs. Our measure of regional urban hilliness, denoted as $R_{i}$, is defined in Table 5.A.3.

Another potentially relevant regional characteristic is the size of a region; since transportation and communication infrastructures are distributed through space, larger regions simply have more room for investment. We therefore include the regional area as an explanatory variable in our specification and denote it as $A_{i}$.

Some country specific variables that vary only over time can also be identified. Investment decisions may be affected by the electoral cycle as suggested by Castells and Sole-Olle (2005): when an election is close, the public investment flows may may be subject to a different decisionmaking process. We include the variable $E_{t}$, defined as the number of years until the next national election year, in order to take into account the effects of the electoral cycle. In order to further capture the countrywide but region-invariant political structure, we introduce a single party dummy, $S_{t}$, which takes the value of one if the national government has a non-coalition single party structure. As a result, $S_{t}$ takes the value of one for the years in which the national government was consisted of a single party (2003 onwards) and zero for the coalition years 1999 through 2002. These variables enter equation (5.1) through the terms $c_{i}$ and $c_{t}$ as follows:

$$
\begin{aligned}
& c_{i}=\rho_{1} A_{i}+\rho_{2} R_{i}+\tilde{c_{i}} \\
& c_{t}=\delta_{1} S_{t}+\delta_{2} E_{t}+\tilde{c_{t}}
\end{aligned}
$$

where $\rho_{1}, \rho_{2}, \delta_{1}$ and $\delta_{2}$ are the parameters associated to the corresponding 
variables and $\tilde{c_{i}}$ and $\tilde{c_{t}}$ are remaining unobserved fixed region and time effects respectively. Substituting to equation (5.1) leads to the empirical specification that we estimate:

$$
\begin{aligned}
\ln I_{i t} & =c_{0}+\beta_{1} \ln I_{i, t-1}+\beta_{2} \ln O_{i t}+\beta_{3} \ln \left(\frac{Y_{i t}}{N_{i t}}\right)+\beta_{4} \ln N_{i t}+\beta_{5} \ln P_{i, t-1} \\
& +\beta_{6} G_{i, t-1}+\beta_{7} \ln \left(\frac{V_{i t}}{N_{i t}}\right)+\rho_{1} A_{i}+\rho_{2} R_{i}+\delta_{1} S_{t}+\delta_{2} E_{t}+\tilde{c_{i}}+\tilde{c_{t}}+e_{i t}
\end{aligned}
$$

It is important to note that in equation (5.2) only one out of the three political variables vary by region $\left(\ln P_{i, t-1}\right)$. The years remaining to election and the single-party dummy are in national scale and vary over time but are constant across regions. As the variable $\ln P_{i, t-1}$ is the only one that takes into account regional political differences, we expect that this variable should be more significant regarding the allocation decisions than the other two political factors.

As earlier discussed, there may exist endogeneity between $I_{i t}$ and $O_{i t}$ due to a potential simultaneity problem: investments in different sectors that a regions receives may depend on each other. Therefore we will begin by instrumenting $\ln O_{i t}$ with its lagged value $\left(\ln O_{i t-1}\right)$ and estimate the model with two-stage least squares with region specific fixed effects (IV-FE). ${ }^{17}$ On the other hand, another source of endogeneity is due to the presence of the lagged dependent variable. In order to account for this endogeneity, we estimate equation (5.2) with the Arellano and Bond (1991a) estimator and the Arellano and Bond (1991b)/Blundell and Bond (1998) estimator as well, also called Difference GMM (Diff-GMM) and System GMM (Sys-GMM) respectively. ${ }^{18}$ The variable $\ln O_{i, t}$ is again treated as an endogenous variable in the GMM estimations, but this time it is instrumented in GMM fashion as discussed in Arellano and Bond (1991a,b); Blundell and Bond (1998); Roodman (2009). We prefer

\footnotetext{
${ }^{17}$ The IV-FE estimation is made using the XTIVREG2 command in stata developed by Schaffer (2005).

${ }^{18}$ The GMM estimations have been done in STATA 13 by using the XTABOND2 command developed by Roodman (2009).
} 
the difference and system-GMM models over the IV-FE estimation as they are the suitable methods for dealing with the bias caused by the lagged dependent variable. Finally, in the GMM estimations, we include the lagged industrial electricity consumption per capita, $K_{i, t-1}$, as an IV style instrument. The results of our estimations and their discussion are presented in Section 5.5.

\subsection{Data and Descriptive Statistics}

\subsubsection{Presenting a modified output and public investment series for Turkish regions}

This study is based on an extensive collection of Turkish regional output data. The official regional output and public investment data on Turkish regions are subject to certain limitations. Especially, the fragmented and inconsistent nature of the regional output data imposes limitations on the time span a study can cover.

By the time this study was finalized, a province level (NUTS 3) GDP series existed for Turkey between 1987 and 2001. Separately, a regional level (NUTS 2) GVA series existed for the years between 2004 and 2011. There was no sub-national output data for 2002 and 2003. However, these two series were presented as part of one single regional Gross Value Added series for the period 1995-2006 by EUROSTAT until the second half of 2011, and by $O E C D$ Stat until March $2013 .{ }^{19}$ We constructed a unique regional GVA series for Turkey in order to cope with this limitation as follows: after aggregating all the data to NUTS 2 level and deflating the figures such that all are in 1998 prices, we imputed the missing years and expressed the entire series in terms of GVA. As a

\footnotetext{
${ }^{19}$ The figures for the years 2002 and 2003 were accurately left blank. Both institutes removed this data upon our notification that the two series could not be treated as one. We would like to thank the officials at TURKSTAT for confirming this situation, and to officials from EUROSTAT and OECD Stat for removing the previously published data.
} 
result we obtained a regional GVA series for each 26 NUTS 2 regions of Turkey for the period between 1987 through 2011. ${ }^{20}$ Therefore, this study presents a reconciled regional output series for Turkey for the period 1987-2011, and suggests the use of this adjusted data set in future research on the Turkish regional economies. ${ }^{21}$

An additional problem was that the regional public investment data reported only province exclusive investments but not investments that are shared among provinces. In order to address this issue, we modified the public investment data of SPO as follows: firstly, a currency change in Turkey which "erased six zeros" from the Turkish Lira in the mid 2000 's was accounted for. Secondly, as in the original source this data is in provincial level, we aggregated the spatial units to NUTS 2 level. On the other hand, the public investments that are directed to more than one province were not recorded in the original data provided by the SPO. However, these "missing" figures were reported under a "Multifarious Provinces" classification for each year where individual projects and the specific locations where investments are directed were listed. ${ }^{22}$ As an exploratory exercise, we have transformed the data by distributing these investments to "multifarious provinces" into the corresponding provinces following a tedious data cleaning process for the three most populous provinces of Turkey in order to attain more precise figures. We observed that the transformed data compared to the original version caused roughly an upward shift of the trend lines of public investments in transportation and communication. Therefore, the third step in our modification of the public investments data was distributing these

${ }^{20}$ Section 5.A.1 presents the modifications done to generate a complete regional GVA series for the range 1987-2011. We are aware of at least one study published in a peer reviewed journal, Onder and Ozyildirim (2011), that uses regional GDP data for Turkey for the period 1992-2006, but does not mention the issue of inconsistency and gaps in the available series.

${ }^{21}$ Any new regional GVA data published in the future can be integrated straightforwardly using our methodology in order to start the regional accounts series from as far back as 1987 instead of 2004.

${ }^{22}$ For public investments in transportation and communication, this process was feasible as location names are specified for almost all projects. However, such an approach would be almost impossible for the investment figures in other sectors as by the time this study was completed - the "Multifarious Provinces" section provided little geographic information regarding the other sectors of public investment. 
"multifarious investments" to all regions (for each year in the sample) by inflating the province specific investments by the ratio of the amounts that were not reported as region-specific. ${ }^{23}$

\subsubsection{Infrastructure stock data}

The descriptive statistics of the variables used for the estimations and in the construction of the infrastructure index are detailed in Table 2.1. In Tables 5.A.4 to 5.A.8, the public investment flows in transportation and communication are compared to the percentage changes in the infrastructure index, road, highway, railroad lengths, and to the air passenger capacities for the years 1999 through 2011 for each of the five economically largest regions. ${ }^{24}$ These tables show that there are years where the stock measurements included in our data are non-responsive to monetary investment. This could be due to the fact that stock variables measure infrastructural attributes such as length and capacity while the monetary investment figures measure additional attributes that are not reflected by stock measures. These are expenditures such as maintenance and repairs, IT updates, re-ordering, modernization, and reinforcement of previously built infrastructure as specified in the relevant governmental database. ${ }^{25}$ This also brings up the question on how well $G_{i t}$ represents infrastructure stock. The tables show that negative percentage changes are present for land transportation infrastructure indicators. This is can be due to shortening road distances in some cases. Examples of roads that decreased in length during the last decade and their amounts of decrease as documented by governmental sources are Adıyaman - Ankara

\footnotetext{
${ }^{23}$ The details of this process are presented in Section 5.A.2.

${ }^{24}$ There are some figures that stand out in Tables 5.A.6 and 5.A.7 which may be explained as follows: in Bursa, a construction investment for a $190 \mathrm{~km}$ "High standard railroad" between Bandirma, Bursa, and Bilecik was done in 2009 (Devlet Planlama Teskilati, a), while in Ankara, investment for a high-speed train between Ankara and Konya was made in 2009 (Devlet Planlama Teskilati, a), and investments were made for multiple railroad constructions from Ankara to many other destinations in 2010 (Devlet Planlama Teskilati, b).

${ }^{25}$ The database of the Republic of Turkey, Ministry of Development (2012). Retrieved on 3-10-2012 from under http://www2.dpt.gov.tr/kamuyat/il.html.
} 
$(50 \mathrm{~km}),{ }^{26}$ Artvin - Erzurum $(24 \mathrm{Km}),{ }^{27}$ Black Sea Coastal Road (17 $\mathrm{km}),{ }^{28}$ and a $1.5 \mathrm{~km}$ decrease in distance due to opening of new tunnels on the Antalya-Kemer-Tekirova road. ${ }^{29}$

Therefore, investment may correspond to the removal of infrastructure stock (i.e larger stocks of infrastructure being replaced by smaller, more efficient improvements). While such negative observations are about 0.38 of the total observations of changes in $G_{i t}$ in our data, the amount of these negative changes account for only about $15 \%$ of the total change in $G_{i t}$. Therefore, in interpreting the results of our analysis, we do not depart from the common interpretation of an infrastructure index: a higher stock represents better infrastructure. This implies that, as discussed earlier, a negative sign on this variable (at time $t-1$ ) would provide evidence that a lower stock of regional infrastructure is expected to result in higher investment flows in the next period. This would imply that the the policy target is to focus on regions lagging in terms of infrastructure rather than those with higher endowment. On the other hand, the presence of such negative values is a potential drawback regarding any study that uses stock measures of infrastructure and requires careful assessment before interpretations using such variables are made.

\footnotetext{
${ }^{26}$ Governorship of Adlyaman, retrieved on 3-10-2012 from http://www.adiyaman.gov.tr/ortak_icerik/adiyaman .icisleri/dosyalar/devam_edenY1.pdf.

${ }^{27}$ Governorship of Artvin, retrieved on 3-10-2012 from http://www.artvin.gov.tr/index.php?page=haber\&file $=$ detay\&id $=9700$.

${ }^{28}$ Republic of Turkey, Ministry of Transport, Maritime Affairs and Communications (2012). Retrieved on 3-10-2012 from http://www2.tbmm.gov.tr/d24/7/7-4158sgc. pdf.

${ }^{29}$ Republic of Turkey, Ministry of Transport, Maritime Affairs and Communications. Retrieved on 3-10-2012 fromhttp://www.kgm.gov.tr/Sayfalar/KGM/SiteEng/Root/ MainPageEnglish.aspx.
} 
TABLE 5.1

DESCRIPTIVE STATISTICS

\begin{tabular}{|c|c|c|c|c|}
\hline Variable & Mean & $\begin{array}{l}\text { Std. } \\
\text { Dev. }\end{array}$ & Min. & Max. \\
\hline $\begin{array}{l}\text { Public investments in transporta- } \\
\text { tion and communication, millions } \\
\text { of TL. }\end{array}$ & 37.52 & 87.23 & 0.23 & 711.37 \\
\hline $\begin{array}{l}\text { Other public investments, millions } \\
\text { of TL. }\end{array}$ & 87.8 & 66.32 & 1.51 & 406.98 \\
\hline Gross value added per capita, TL. & 952.64 & 421.84 & 349.04 & 2069.78 \\
\hline Population (Millions). & 2.71 & 1.98 & 0.73 & 13.26 \\
\hline $\begin{array}{l}\text { Share of region's MP's in the gov- } \\
\text { ernment in total MP's of the region. }\end{array}$ & 0.64 & 0.15 & 0.24 & 0.93 \\
\hline Infrastructure index. & 7.04 & 2.44 & 4 & 12.22 \\
\hline Vehicles per 1000 capita. & 115.27 & 60.17 & 18.48 & 279.35 \\
\hline Single party dummy. & 0.69 & 0.46 & 0 & 1 \\
\hline Years remaining to election. & 1.46 & 1.28 & 0 & 4 \\
\hline Area, 1000 sqm's. & 29.6 & 12.06 & 5.2 & 59.66 \\
\hline Roughness measure. & 54.72 & 39.6 & 1.17 & 118.26 \\
\hline $\begin{array}{l}\text { Industrial electricity consumption } \\
\text { per capita, KWh. }\end{array}$ & 0.92 & 0.88 & 0.03 & 3.93 \\
\hline $\mathrm{N}$ & \multicolumn{4}{|c|}{338} \\
\hline
\end{tabular}

\subsection{Empirical Results}

The estimation results are reported in Table 5.2. Year dummies are included in all models. We observe that all three estimation methods find a significant impact of the political affinity of the regions to the 
central government $\left(\ln P_{i, t-1}\right)$ on the amount of public in investments in transportation and communication they receive. This result is the main finding of our study: regions with a higher share of government MP's have received higher transportation and communication investments, while those with less political affinity to the government party received less investments in this category of infrastructure through 1999 and 2011. Therefore, according to our results, political effects play a significant role in the allocation of regional transportation and communication infrastructure in Turkey.

The results of all three models also suggest that dynamic effects are present in the regional public investment flows. In other words, previous investments have a "spillover" effect to the current period. This result is robust throughout our models and is supported by the AR1 test results from the difference and system GMM estimations in the second and third columns respectively. As discussed in Section 5.3, the continuity of regional investment policies and the interrelatedness of investment flows in consecutive periods may give rise to this outcome. On the other hand, the other types of public investments a region receives $\left(O_{i t}\right)$ does not have a significant coefficient. However, the consistent negative sign that it has in the results from all our models may hint at a substitution effect rather than a complementary one.

All our models provide evidence that the higher the efficiency (per capita output) of a region is, the higher are investments in transportation and communication. This result is especially prominent based on the IV-FE results. However, this effect diminishes both in magnitude and significance as the endogeneity posed by the lagged dependent variable is taken into account in the GMM models presented in columns 2 and 3.

Population has a significant impact based on the IV results and system GMM estimation. According to the system-GMM estimation, a 1\% increase in population increases investments by around $0.7 \%$ while the predicted approximate increase in investment as a response to a $1 \%$ increase in per capita output is about 1.1\% (significant only in the $10 \%$ level). The coefficients of $\ln \left(G V A_{i t} / N_{i t}\right)$ are also higher in the first two columns than those of $\ln N_{i t}$. Therefore, except the difference-GMM 
results, our findings imply that both these regional attributes play a role in the investment decisions, and regional efficiency seems to have a relatively larger effect than regional population. While we find that the efficiency-equity trade-off is slightly in favor of efficiency, we refrain from making a clear suggestion that the government clearly values national efficiency over national equity due to the low significances and small differences between the two estimates (except for the IV-FE model).

Aside from $\left(P_{i, t-1}\right)$, out of the other two political variables, only $S_{t}$ (single party dummy) yields a significant coefficient as the System-GMM results suggest that regions received higher investments in transportation and communication during this period relative to the prior coalition years. However, while this result is highly significant in the System-GMM model, it is not supported in the first and second columns of Table 5.2. Furthermore, while the number of years to an election does not yield significant coefficients, it is estimated with a negative sign in all three columns. If significance was observed, this result would have suggested that as elections get closer, regional investments increase.

The IV-FE and difference-GMM models suggest that the effect of previously existing regional infrastructure $\left(G_{i t-1}\right)$ is negative. Based on the discussion regarding this variable in Section 5.4, this would mean that regions with less infrastructure receive higher infrastructure investment. In other words, a region is expected to receive higher investments in transportation and communication in the future period if they have relatively less stock of this type of infrastructure in the current period. The implication is that policy makers are concerned with infrastructure deprivation and prioritize regions lagging in infrastructure stock. On the other hand, this result is not supported by the system-GMM estimation. Similarly, congestion $\left(V_{i t} / N_{i t}\right)$ does not seem to play a role in the allocation decisions.

Finally, according to our results geography is not a determinant of the allocation decisions: neither the size or the hilliness of a region yield significant estimates. 
TABLE 5.2

ESTIMATION RESULTS FOR EQUATION (10)

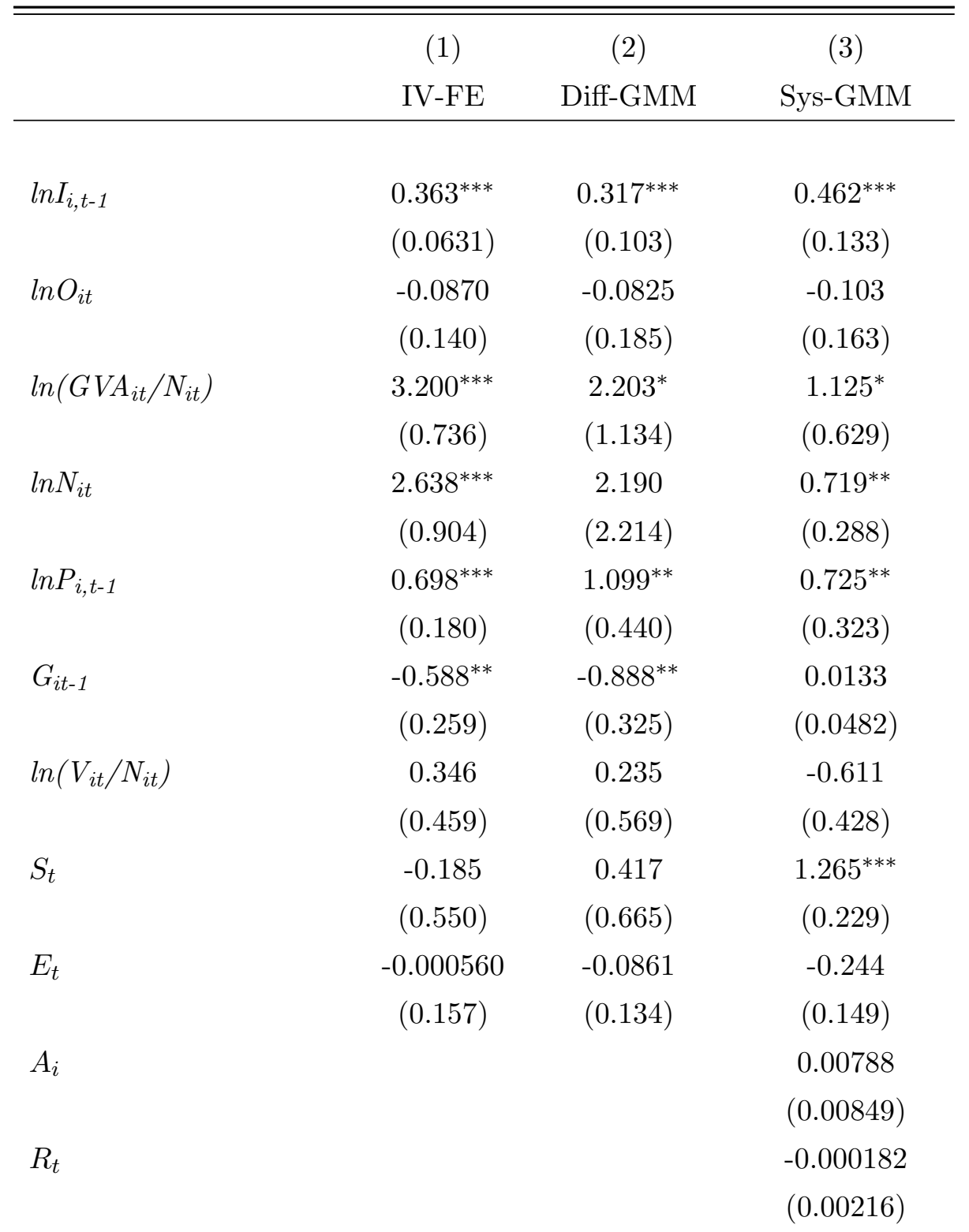


TABle 5.2

Estimation Results for Equation (10) (CONT'D)

(1)

(2)

(3)

\begin{tabular}{lccc} 
& IV-FE & Diff-GMM & Sys-GMM \\
\hline Constant & & & -13.81 \\
& 312 & 286 & $(8.837)$ \\
\hline Observations & 26 & 26 & 312 \\
Number of regions & 12 & 11 & 26 \\
Observations per region & 1 & 21 & 12 \\
Number of instruments & & $(1,2)$ & 26 \\
GMM lag limits & $\ln O_{i t}$ & $\ln (I)_{i, t-1}, \ln O_{i t}$ & $\ln (I)_{i, t-1}, \ln O_{i t}$ \\
Instrumented variable(s) & & 0.000343 & 0.000278 \\
AR1 test (p-value) & & 0.724 & 0.872 \\
AR2 test (p-value) & & 0.590 & 0.221 \\
Hansen test (p-value) & & 0.650 & 0.136 \\
Sargan test (p-value) & & Yes & Yes \\
Year Dummies & Yes & & \\
& & &
\end{tabular}

Standard errors in parentheses: ${ }^{*} p<0.10,{ }^{* *} p<0.05,{ }^{* * *} p<0.01$ 


\subsection{Concluding Discussion and Policy Implica- tions}

The main outcome of this study is the observed strong evidence that political influences have been present in the allocation decisions of regional transportation and communication investments in Turkey through the period 1999 - 2011. Elaborating on this result by considering the various economic benefits presented by this category of infrastructure, we argue that the regional allocation of transportation and communication infrastructure in Turkey is not efficiently conducted. A diversion of regional policies - caused by political influences - from their goals of economic efficiency or equity could result in a lower level of welfare not only for regions, but also for the national economy as a whole. The main policy recommendation resulting from this study is to reduce the political effects arising from the political connections between regions and the central authority from this decision-making process in the favor of concentrating on national economic goals such as the efficiency or equity of regions. Our results provide evidence to actors who can contribute to this process. NGO's, opposition parties, and economic agents in general that operate in regions that suffer the negative results of these political influences can pressure policy-makers for a less politically biased process of regional public capital allocation. Our results could help the discussion on whether or not this influence exists (and how strong it is) to become more than a media-based debate by providing academic evidence.

In the context of a possible dependence between infrastructure types, we had assumed that investments in transportation and communication share the same government resource constraint with other types of investments. However, no evidence for the complementability or substitutabilty between these infrastructure categories was observed. Nevertheless, we believe further evidence is necessary - including industry specific research on the other categories of public investment such as health and education - as allocation behavior may differ based on sectors. 


\section{A Appendix}

\section{A.1 Gross Value Added Data Adjustments}

As earlier mentioned, the output indicators for Turkish regions do not follow a comparable structure for the data range used in this study. Therefore, we modified the available data as follows: First, the province level data was aggregated so that the spatial units would correspond to NUTS2 regions. Next, the series was deflated to 1998 constant national currency. The output series which is in terms of GDP for 1987-2001 and in terms of GVA for 2004-2011 (thus, with a two-year gap) is labeled as "R_Output," the national GDP series for Turkey as a whole for the whole range is labeled as " $N_{-}$Output." A dummy variable that takes the value of one if the year is between 2004-2011 (i.e. if the measured regional output is in terms of GVA), is labeled as "GVAdum."

In order to express all the series in terms of GVA, for each region, the below pooled OLS regression was estimated for the years in the sample before 2004:

$$
\ln \left(R_{-} \text {Output }\right)_{t}=\beta_{0}+\beta_{1} \ln \left(N_{-} \text {Output }\right)_{t}+\beta_{2} \text { GVAdum }{ }_{t}+\epsilon_{t}
$$

Using the parameter estimates from equation (5.3), ln regional GVA $\left(\ln R G V A_{t}\right)$ in constant 1998 currency was predicted. Finally, the already existing regional GVA figures for 2004-2011 were merged into this predicted series. As a result, a reconciled and interpolated regional NUTS2 GVA series for 1987-2011 has been available for the analyses conducted in this study.

\section{A.2 Public Investments Data Adjustments}

By the time our data collection was completed, data on public investments were available in provincial level (NUTS3) for 1999-2011. As for the GVA adjustments, the provincial data has been first aggregated to 
regional (NUTS2) scale. The original source reports provincial data only if all investment has been directed within the province. In other words, data is not recorded in a province-specific manner if investments were directed to more than one province. For example, investments on a road connecting two or more provinces did not appear within the category of any of those provinces. However, such investments are reported under a "Multifarious Provinces" category, where the investment and the directed provinces are explained in detail. For the three large provinces (Istanbul, Izmir, and Ankara), we have distributed the investments by hand from the "Multifarious Provinces" category to the three provinces. When investments were specified as directed to more than one province, we weighted all those provinces equally when distributing the investment figures, as it is impossible to know how project investments were shared among them. As a result, we were able to compare the original data to our modified data for the three major provinces. Transportation and communication investment trend lines for each province were shifted upwards, mostly following very similar trends, as can be seen in the below graphs. 
FiguRe 5.A.1

COMPARISON OF ORIGINAL AND MODIFIED DATA

(A) Istanbul

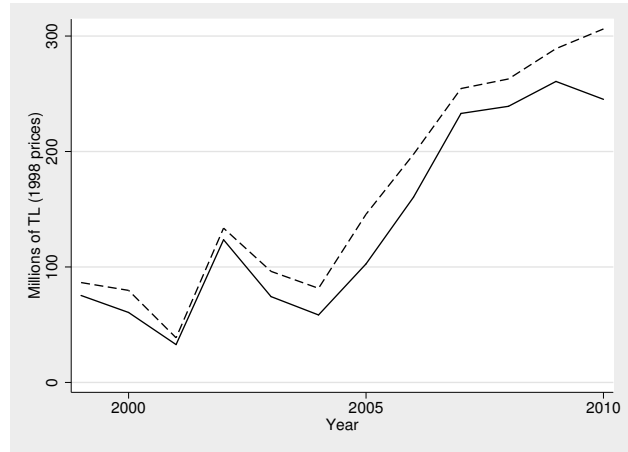

(c) Ankara

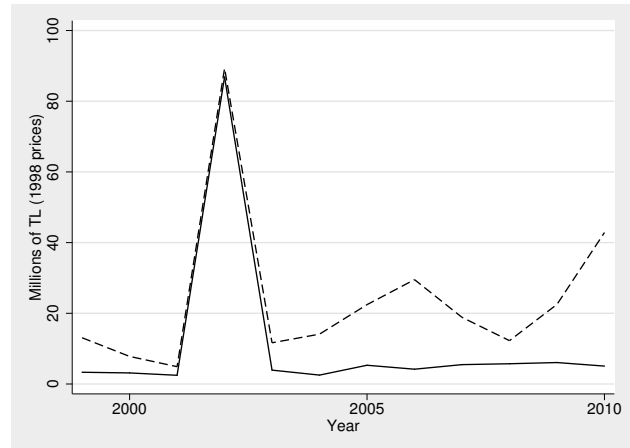

(B) IZMIR

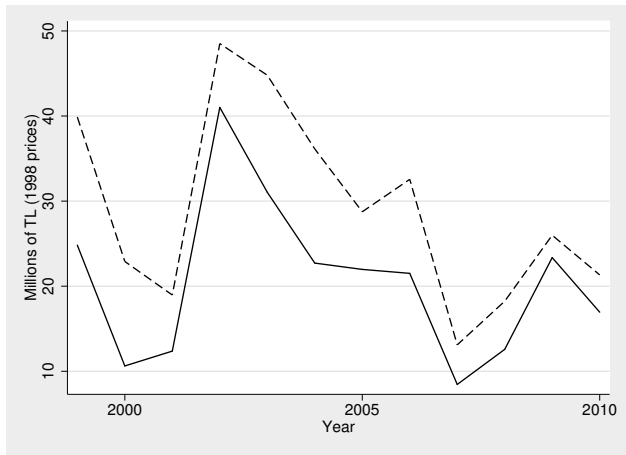


Therefore, upon deflating all investment figures to 1998 prices in national currency using the GDP deflator for 1998, we have made an assumption that is possible to inflate each province's investment figures by the recorded figures times the ratio of the missing investments for each year. The details of this modification are as follows:

If we call the

- transportation and communication investments that are summed from available provincial data "Recorded TPI,"

- reported aggregate country investments in transportation and communication where no investment figure is missing, "Aggregate TPI," and

- the originally available provincial investment data "OTPI,"

and if for each year,

- Aggregate TPI - Recorded TPI = Missing TPI, i.e. those that are not showing up under provincial categories but are "hidden" under "Multifarious Provinces," and

- Missing Ratio = Missing TPI / Recorded TPI,

then for each region, the investment figures used in this study $(T P I)$ equals:

OTPI + OTPI $\times$ MissingRatio

The resulting regional figures add up to the officially reported total country aggregate investment in transportation and communication for each year. 
TABLE 5.A.1

REGION CODES AND NAMES

TR10: Istanbul

TR21: Tekirdag, Edirne, Kirklareli

TR22: Balikesir, Canakkale

TR31: Izmir

TR32: Aydin, Denizli, Mugla

TR33: Manisa, Afyon, Kutahya, Usak

TR41: Bursa, Eskisehir, Bilecik

TR42: Kocaeli, Sakarya, Duzce, Bilecik

TR51: Ankara

TR52: Konya, Karaman

TR61: Antalya, Isparta, Burdur

TR62: Adana, Mersin

TR63: Hatay, Kahramanmaras, Osmaniye

TR71: Kirikkale, Aksaray, Nigde,

TR72: Kayseri, Sivas, Yozgat

TR81: Zonguldak, Karabuk, Bartin

TR82: Kastamonu, Cankiri, Sinop

TR83: Samsun, Tokat, Corum, Amasya

TR90: Trabzon, Ordu, Giresun, Rize

TRA1: Erzurum, Erzincan, Bayburt

TRA2: Agri, Kars, Igdir, Ardahan

TRB1: Malatya, Elazig, Bingol, Tunceli

TRB2: Van, Mus, Bitlis, Hakkari

TRC1: Gaziantep, Adiyaman, Kilis

TRC2: Sanliurfa, Diyarbakir

TRC3: Mardin, Batman, Sirnak, Siirt 
TABle 5.A.2. Measurement of POlitical AFFiliation in Other STUDIES

$\begin{array}{lll}\text { Author } & \text { Title } & \text { Measurement } \\ \text { Cadot et al. (1999) } & \begin{array}{l}\text { A Political Economy Model of } \\ \text { Infrastructure Allocation: An } \\ \text { Empirical Assessment }\end{array} & \begin{array}{l}\text { Dummy equal to 1 when the } \\ \text { majority in a regional council } \\ \text { and that of the national par- } \\ \text { liament are either both right- } \\ \text { wing or both left-wing. }\end{array}\end{array}$

Costa-I-Font et al. Political competition and pork(2003) barrel politics in the allocation of public investment in Mexico

The share of votes in the municipalities received by the governing party in each state, A dummy variable for states governed by a political party different from the governing party.

Castells and Sole-Olle The regional allocation of in(2005) frastructure investment: The role of equity, efficiency and political factors

Moré and Ollé (2005) Does decentralization improve the efficiency in the allocation of public investment? Evidence from Spain

Kemmerling and Bo- Partisan Politics in Regional denstein (2006) Redistribution Do Parties Affect the Distribution of EU Structural Funds across Regions?

Sole-Olle and SorribasNavarro (2008)

The effects of partisan alignment on the allocation of intergovernmental transfers. Differences-in-differences estimates for Spain
Numerous variables constructed from data on election results, election system, and other political characteristics.

The incumbent party's vote share in the last election.

Size of the left and eurosceptic parties.

The relative policital position of the grant receiving government (partner, leader, etc. of the upper and lower level governments) or the difference between the vote share of the party in government and the vote share of the second party. 
Table 5.A.2. Variable Measurement of political affiliation IN OTHER STUDIES (CONT'D)

\begin{tabular}{|c|c|c|}
\hline Author & Title & Measurement \\
\hline $\begin{array}{l}\text { Mizutani and Tanaka } \\
(2008)\end{array}$ & $\begin{array}{l}\text { Productivity effects and deter- } \\
\text { minants of public infrastruc- } \\
\text { ture investment }\end{array}$ & $\begin{array}{l}\text { Ratio of majority vote to mi- } \\
\text { nority vote in the House of Rep- } \\
\text { resentatives, or percentage of } \\
\text { votes for the gov't party in the } \\
\text { prefectureal congress (depend- } \\
\text { ing on the investment source). }\end{array}$ \\
\hline Zheng et al. (2013) & $\begin{array}{l}\text { Central government's infras- } \\
\text { tructure investment across Chi- } \\
\text { nese regions: A dynamic spa- } \\
\text { tial panel data approach }\end{array}$ & $\begin{array}{l}\text { Number of committee mem- } \\
\text { bers (or candidates) each } \\
\text { province has in the Central } \\
\text { Committee of the Communist } \\
\text { Party of China. }\end{array}$ \\
\hline
\end{tabular}

TABLE 5.A.3

VARIABLE DEFINITIONS

\begin{tabular}{|c|c|c|}
\hline Name & Year Coverage & Description \\
\hline$I_{i t}$ & 1999-2011 & $\begin{array}{l}\text { Public investments in transportation and com- } \\
\text { munication deflated to } 1998 \text { prices (national } \\
\text { currency). Inflated to account for the missing } \\
\text { amounts due to the "Various Provinces" classi- } \\
\text { fication. Source: Republic of Turkey, Ministry } \\
\text { of Development. }\end{array}$ \\
\hline$O_{i t}$ & 1999-2011 & $\begin{array}{l}\text { Public investments in areas other than trans- } \\
\text { portation and communication deflated to } 1998 \\
\text { prices (national currency). Inflated to account } \\
\text { for the missing amounts due to the "Various } \\
\text { Provinces" classification. Source: Republic of } \\
\text { Turkey, Ministry of Development. }\end{array}$ \\
\hline$P_{i t}$ & 1999-2011 & $\begin{array}{l}\text { Number of members of parliament (MP) in } \\
\text { the government from the region divided by the } \\
\text { lagged total number of MP's allocated to the } \\
\text { region. Source for the base variables: Turkish } \\
\text { Statistical Institute (Turkstat). }\end{array}$ \\
\hline$S_{t}$ & 1999-2011 & $\begin{array}{l}\text { Dummy variable that equals one if a single } \\
\text { party government was in power, and equals } \\
\text { zero if a coalition government was in power. }\end{array}$ \\
\hline
\end{tabular}


TABLE 5.A.3

VARIABLE DEFINITIONS (CONT'D)

\begin{tabular}{|c|c|}
\hline Name & Year Cover \\
\hline$Y_{i t}$ & $1987-2011$ \\
\hline$N_{i t}$ & $1990-2011$ \\
\hline$R_{i}$ & Constant \\
\hline$A_{i}$ & Constant \\
\hline$V_{i t}$ & $1990-2011$ \\
\hline$E_{t}$ & $1999-2011$ \\
\hline$K_{i t}$ & $1995-2011$ \\
\hline$G_{i t}$ & $1995-2011$ \\
\hline Road density ${ }_{i t}$ & $1995-2011$ \\
\hline Hway density ${ }_{i t}$ & $1995-2011$ \\
\hline Railroad density ${ }_{i t}$ & $1995-2011$ \\
\hline Air capacity ${ }_{i t}$ & $1987-2011$ \\
\hline
\end{tabular}

Description

Regional Gross value added in 1998 prices (national currency). Source: Turkstat. Modified as specified in Appendix A.

Population. Source: OECD Stat.

Elevation of the highest provincial center of the region minus that of the lowest, divided by regional area. The elevations of the provincial centers in each region has been obtained using the Google Earth software search box. Source: Google Earth 7.0.3.8542.

Area in 1000 square meters, excluding lakes. Source: Turkstat.

Total number of vehicles except trailers or tractors. Source: Eurostat.

The number of years remaining to a year in which elections took place. Takes the value of zero if a given year is an election year.

Electricity consumption by industrial establishments (MWh). Source: Turkish Statistical Institute. Divided by population. Only used as an instrument in the estimations.

Index of transportation and communication infrastructure stock constructed using the first principal components of the variables $\ln$ Road density, ln Hway density, In Railway density, ln total length of public piers, In Air transport capacity.

Provincial road length $(\mathrm{km})$. Source: Turkstat. Divided by Area.

Highway length $(\mathrm{km})$. Source: Turkstat. Divided by Area.

Railroad length $(\mathrm{km})$. Source: Turkstat. Divided by Area.

Total passenger capacity in the regional airports. Compiled from the information on area and establishment dates available at the airport interactive map at the website of the Republic of Turkey: Ministry of Transport, Maritime Affairs and Communication. 
TABLE 5.A.3

VARIABLE DEFINITIONS (CONT'D)

\begin{tabular}{lll}
\hline Name & Year Coverage & Description \\
Pub. pier & as of 2005 (constant) & $\begin{array}{l}\text { Total public pier length (m). Source Repub- } \\
\text { lic of Turkey - Ministry of Transport, Mar- } \\
\text { itime Affairs and Communication "1995 - 2005 }\end{array}$ \\
& Ulaştırma ve Haberleşme", Ankara 2005. \\
ADSL & Table Number of ADSL lines in the PTT of- \\
& fices. Source: Republic of Turkey - General \\
& Directorate of PTT. \\
\hline
\end{tabular}


TABLE 5.A.4

CORRESPONDENCE OF INFRASTRUCTURE INVESTMENT

AND CHANGES INFRASTRUCTURE STOCK, ISTANBUL

\begin{tabular}{ccccccc}
\hline $\begin{array}{c}\text { TR10: Istanbul } \\
\text { Year }\end{array}$ & TPI & $\% \Delta G$ & $\begin{array}{c}\% \Delta \text { Road } \\
\text { length }\end{array}$ & $\begin{array}{c}\% \Delta \\
\text { Highway } \\
\text { length }\end{array}$ & $\begin{array}{c}\% \Delta \\
\text { Railway } \\
\text { length }\end{array}$ & $\begin{array}{c}\% \Delta \text { Airport } \\
\text { capacity }\end{array}$ \\
\hline 2000 & 382969824.0 & 0 & 0 & 0 & 0 & 0 \\
2001 & 167828656.0 & -0.0574539 & -0.4983389 & 0 & 0 & 14 \\
2002 & 213587648.0 & -0.4102019 & -10.35058 & 0 & 0 & 0 \\
2003 & 257194000.0 & 0.2213662 & -2.793296 & 7.612457 & 0 & 0 \\
2004 & 236313232.0 & -0.0238267 & 2.490422 & -2.572347 & 0 & 0 \\
2005 & 354826336.0 & -0.4317256 & -10.84112 & 0 & 0 & 0 \\
2006 & 430711040.0 & -0.6652262 & -16.14256 & 0 & 0 & 0 \\
2007 & 500065440.0 & 0 & 0 & 0 & 0 & 0 \\
2008 & 566248384.0 & -0.1564413 & -0.25 & 0 & -5.238095 & 0 \\
2009 & 647192448.0 & 0 & 0 & 0 & 0 & 0 \\
2010 & 711365696.0 & 0.9425969 & 28.07018 & 0 & 0 & 0 \\
\hline
\end{tabular}


TABLE 5.A.5

CORRESPONDENCE OF INFRASTRUCTURE INVESTMENT AND CHANGES INFRASTRUCTURE STOCK, IZMIR

\begin{tabular}{ccccccc}
\hline \multicolumn{7}{c}{ TR31: Izmir } \\
Year & TPI & $\% \Delta G$ & $\begin{array}{c}\% \text { Road } \\
\text { length }\end{array}$ & $\begin{array}{c}\% \Delta \\
\text { Highway } \\
\text { length }\end{array}$ & $\begin{array}{c}\% \Delta \\
\text { Railway } \\
\text { length }\end{array}$ & $\begin{array}{c}\% \Delta \\
\text { Airport } \\
\text { capacity }\end{array}$ \\
\hline 2000 & 67156928.0 & 0.0137332 & -1.570681 & 1.704545 & 0 & 0 \\
2001 & 63317432.0 & 0 & 0 & 0 & 0 & 0 \\
2002 & 70885456.0 & 0.0891208 & -0.987842 & 2.793296 & 0 & 0 \\
2003 & 107371784.0 & 0.3773066 & -0.5372218 & 3.26087 & 8.695652 & 0 \\
2004 & 91823424.0 & 0.0483963 & 1.774691 & -0.5263158 & 0 & 0 \\
2005 & 75945392.0 & -0.0221887 & -0.5307051 & 0 & 0 & 0 \\
2006 & 57746812.0 & 0.2745712 & 0.152439 & 5.820106 & 0 & 0 \\
2007 & 18143602.0 & 0 & 0 & 0 & 0 & 0 \\
2008 & 29777156.0 & 0.169755 & -1.674277 & 4 & 1.846154 & 0 \\
2009 & 57994792.0 & 0.0162951 & -0.1547988 & .4807692 & 0 & 0 \\
2010 & 49172868.0 & -0.0128944 & -0.3100775 & 0 & 0 & 0 \\
\hline
\end{tabular}


TABLE 5.A.6

CORRESPONDENCE OF INFRASTRUCTURE INVESTMENT AND CHANGES INFRASTRUCTURE STOCK, Bursa

\begin{tabular}{ccccccc}
\hline \multicolumn{7}{c}{ TR41: Bursa } \\
Year & TPI & $\% \Delta G$ & $\begin{array}{c}\% \text { Road } \\
\text { length }\end{array}$ & $\begin{array}{c}\% \Delta \\
\text { Highway } \\
\text { length }\end{array}$ & $\begin{array}{c}\text { Railway } \\
\text { length }\end{array}$ & $\begin{array}{c}\% \Delta \\
\text { Airport } \\
\text { capacity }\end{array}$ \\
\hline 2000 & 119625496.0 & -0.9927337 & 0 & 0 & 0 & 750 \\
2001 & 59517364.0 & -0.0074947 & -0.1258917 & 0 & 0 & 0 \\
2002 & 43827840.0 & 0 & 0 & 0 & 0 & 0 \\
2003 & 104561104.0 & -0.0125159 & -0.210084 & 0 & 0 & 0 \\
2004 & 133385976.0 & 3.810494 & -0.0421053 & 0 & 0 & 0 \\
2005 & 92055936.0 & -0.0048307 & -0.084246 & 0 & 0 & 0 \\
2006 & 97656920.0 & 4.53861 & 0 & 225 & 0 & 0 \\
2007 & 57448380.0 & 0 & 0 & 0 & 0 & 0 \\
2008 & 59269704.0 & -0.1369844 & 0.2107926 & 0 & -3.899721 & 0 \\
2009 & 50056440.0 & 1.910984 & .4627682 & 0 & 64.34782 & 0 \\
2010 & 65583524.0 & 0.7201613 & 1.675042 & 13.84615 & .8818342 & 0 \\
\hline
\end{tabular}


TABLE 5.A.7

CORRESPONDENCE OF INFRASTRUCTURE INVESTMENT AND Changes infrastructure STOCK, AnKara

\begin{tabular}{ccccccc}
\hline \multicolumn{7}{c}{ TR51: Ankara } \\
Year & TPI & $\% \Delta G$ & $\begin{array}{c}\% \text { Road } \\
\text { length }\end{array}$ & $\begin{array}{c}\% \Delta \\
\text { Highway } \\
\text { length }\end{array}$ & $\begin{array}{c}\text { Railway } \\
\text { length }\end{array}$ & $\begin{array}{c}\% \Delta \\
\text { Airport } \\
\text { capacity }\end{array}$ \\
\hline 2000 & 19805366.0 & -0.0787801 & -0.968523 & 0 & 0 & 0 \\
2001 & 12619823.0 & -0.5572711 & -0.3667482 & -5.882353 & 0 & 0 \\
2002 & 150107392.0 & 0.0448568 & 0.5521472 & 0 & 0 & 0 \\
2003 & 13581354.0 & 0.3077955 & -0.0610128 & 3.645833 & 0 & 0 \\
2004 & 10192342.0 & -0.0637925 & -0.2442002 & -0.5025126 & 0 & 0 \\
2005 & 18341684.0 & 0.0099385 & 0.122399 & 0 & 0 & 0 \\
2006 & 11244385.0 & 0 & 0 & 0 & 0 & 0 \\
2007 & 11763313.0 & -0.0498027 & -0.6112469 & 0 & 0 & 0 \\
2008 & 13571400.0 & -0.0150068 & -0.1845018 & 0 & 0 & 0 \\
2009 & 15077028.0 & 2.412661 & 0.6161429 & 0 & 52.25806 & 0 \\
2010 & 14717430.0 & 1.941078 & 1.592162 & 0 & 38.34746 & 0 \\
\hline
\end{tabular}


TABLE 5.A.8

CORRESPONDENCE OF INFRASTRUCTURE INVESTMENT AND CHANGES INFrastruCTURE STOCK, KocAELI

\begin{tabular}{ccccccc}
\hline \multicolumn{7}{c}{ TR42: Kocaeli } \\
Year & TPI & $\% \Delta G$ & $\begin{array}{c}\% \text { Road } \\
\text { length }\end{array}$ & $\begin{array}{c}\% \Delta \\
\text { Highway } \\
\text { length }\end{array}$ & $\begin{array}{c}\% \Delta \\
\text { Railway } \\
\text { length }\end{array}$ & $\begin{array}{c}\% \Delta \\
\text { Airport } \\
\text { capacity }\end{array}$ \\
\hline 2000 & 37998184.0 & -0.1056419 & 0.39801 & 0 & -4.198473 & 0 \\
2001 & 25127580.0 & -0.006224 & -0.148662 & 0 & 0 & 0 \\
2002 & 5258311.5 & -0.07326 & -1.736973 & 0 & 0 & 0 \\
2003 & 8329263.0 & -0.1528016 & -3.585859 & 0 & 0 & 0 \\
2004 & 16884820.0 & 0.0177367 & -0.3143007 & .6535948 & 0 & 0 \\
2005 & 39677976.0 & 0 & 0 & 0 & 0 & 0 \\
2006 & 25727916.0 & 0.3315606 & -0.262743 & 7.467533 & 0 & 0 \\
2007 & 13138195.0 & -0.0021968 & -0.052687 & 0 & 0 & 0 \\
2008 & 23087934.0 & 0.0361985 & -0.2108593 & 0 & 1.593626 & 0 \\
2009 & 14432585.0 & -0.0132495 & -0.3169572 & 0 & 0 & 0 \\
2010 & 20988992.0 & 0.0154567 & 0.3709592 & 0 & 0 & 0 \\
\hline
\end{tabular}




\title{
CHAPTER 6
}

\author{
Conclusion
}

\subsection{A Review of the Research Findings and Their Implications}

By presenting four separate articles, this dissertation aimed to shed further light on the outcomes and determinants of specific regional economic policies, directed particularly towards infrastructure. This was done by seeking answers to the questions: "what types of regional infrastructure policies are useful for achieving regional economic targets in terms of convergence and trade?" and "how are these policies decided in Turkey?"

These questions were posed and tackled empirically by looking at how specific types of connectivity-enhancing infrastructures are allocated among regions in Turkey by central planners, and how these types of infrastructures (in some cases also their relative locations), impact regional convergence and trade. While the convergence and allocation questions were examined in a case specific manner on Turkish regions, the question regarding trade was studied also in more general terms, through meta-analytic methods.

The results of this dissertation can potentially be translated into activities related to the enhancement and location choice of connectivity-related infrastructure, based on specific regional and national needs. In turn, such 
region-specific activities could influence the general economic geography within a national economy as a whole. The results can also support political activities that aim the attainment of an optimal strategy in allocating infrastructure across the regions of Turkey.

More specifically, aside of a meta-analytic study looking at the infrastructure-trade relationship, three studies were presented on infrastructure-related regional policies in Turkey. The focus was on communication and transportation infrastructure. There were several key findings. Firstly, in Chapter 2, internet infrastructure was found to help reduce spatial inequalities in Turkey, which have been a longstanding issue (Gezici and Hewings, 2007). As put in this chapter, the mechanism through which communication infrastructures such as internet infrastructure, impact regional per capita income differences may work through reducing the information gap between regional markets (Carey, 2008), stimulating the flows of capital (Dokmeci and Berkoz, 1996), creating new patterns of regional homogenization (Robins and Gillespie, 1992), homogenizing regional culture and institutions (Breuer et al., 2014), creating productivity spillovers and attracting resources from other regions (Ding et al., 2008), generating locational advantages due to being in digital networks (Tranos and Gillespie, 2009), and influencing the New Economic Geography (NEG) equilibrium through the reduction of the costs of communication (Tranos, 2012).

Secondly, the meta-analytic study presented in Chapter 3 found an interesting result: previous studies persistently estimated that traderelated infrastructures enhance the exports of the home economy more than its imports. The chapter further elaborates on this result which presents important implications in terms of trade balances. Regarding regional exports, further evidence was presented in Chapter 4 on the role of transportation and communication infrastructure for the specific case of Turkey. This chapter distinguished among specific types of traderelated infrastructure, and found the extent to which their presence, density, and location within a region influence regional export flows through the reduction of transportation and communication costs.

Finally, the dissertation has identified in Chapter 5 that political influ- 
ences are present in Turkish regional policy-making. These influences are considered in the dissertation as effects which could potentially distort the equity-efficiency trade-off in the regional allocation of public capital. More specifically, the results of this chapter suggested that regions which are aligned closer to the central government are more likely to receive higher public investments in transportation and communication public infrastructure.

Each of the four studies have presented several new elements in terms of analytical contributions and syntheses, applications of various methodologies on previously non-examined cases, and reconciliations of existing but imperfect official data sets for the use of future research projects. Some major novelties can be discussed in relation to the social and economic relevance of this dissertation. All studies are original in terms of their focuses: for instance, in Chapter 2, a modern type of telecommunication infrastructure was taken into focus in relation to regional convergence, namely, internet infrastructure. The chapter presents the first study which looks at this proposed internet-regional income convergence relationship. The results of this chapter have supported the hypothesis that better internet infrastructure makes regional economies more similar by increasing their speeds of convergence to their steady-state levels of per capita income, and also by making the individual steady-states of regional economies more alike. As a result, for countries like Turkey where infrastructure deprivation is still an issue to some extent, increasing the regional communication ties through modern communication technologies is suggested as a relevant policy tool - if regional disparities are seen as a problem. In terms of scientific relevance, Chapter 2 provided an application of methods of spatial analysis on Turkey. Descriptive and empirical approaches have underlined a significant core-periphery pattern in the country, where poor and rich regions are clustered together.

Furthermore, Chapter 3 presented the first meta-analysis of the impacts of infrastructure on trade. The relationship between infrastructure and trade has attracted much empirical attention since the late 1990's and early 2000's. The results of this chapter suggested that previous research findings imply that infrastructure enhances exports more than imports. This is obviously an outcome that needs further attention. Such 
a result would suggest from a policy making perspective that ceteris paribus, enhancing own trade related infrastructure could help improve the trade balance in an economy. Why such results have been observed in the literature is further discussed in the concluding section of this chapter. In terms of scientific relevance, this chapter presents the first meta-analytic application on the question of the relationship between infrastructure and trade which synthesizes previously observed statistical results by explicitly taking publication bias (or file drawer bias) into account. This is done by following the concrete guidelines for conducting a meta-analysis provided in Stanley et al. (2013)

The gravity model application on Turkish exports to world countries that was presented in Chapter 4 is the first such study for Turkey which is carried out on the sub-national level. The chapter assessed not only the impact of the presence of infrastructure, but also the effects of the location of point infrastructures (e.g. ports and airports) as exit or entry nodes for trade flows. Results have essential implications on policy decisions regarding where to locate infrastructure and what type of infrastructure is good for exports. The chapter underlined the importance of private ports, air transport infrastructure, and land infrastructure for regional export performance. For the purpose of creating a suitable data set for this study, the specific locations (through the use of coordinates) of the airports and ports in Turkish regions were recorded. Moreover, the intensity of most infrastructure types such as airport and port capacities and communication infrastructure statistics were collected. This compiled data set from fragmented resources is also the first of its kind for Turkish regions. Using this data has enabled a deep examination of the effects of infrastructure on trade by not only looking at what type of infrastructure is important for exports, but also by examining how enhancements in these specific types of infrastructures impact regional exports. Moreover, the locations of these types of infrastructures relative to the major provincial centers within regions were examined. As a novel approach for Turkish regional research, the average distances between the largest urban agglomerations in the regions and closest point infrastructures such as airports and ports were put into focus. For instance one relevant implication that resulted from the usage of this data set, was that in Turkey, the locations of airports have important 
effects on regional exports.

Finally, the study presented Chapter 5 which looks at the determinants of the allocation of regional transportation and communication infrastructure is the only study that recognizes the dependency between allocation decisions of different infrastructure types. Again taking Turkey as the country of focus, it is a key chapter as this dissertation does not only look at how transportation and communication public infrastructure affects convergence and trade, but also at how it is allocated by the central planners. This is especially relevant for regional policy making and its applications. It was assumed, in line with the prominent theories in the literature, that there are two main points of interest in the decision making process regarding the allocation of public capital: national efficiency and regional equity. The policymakers, depending on their purposes, allocate weights on these goals in various degrees. However, this mechanism of preference may be influenced by other factors, mainly, political ones. This chapter shows that in Turkey, the regional allocation of investments in transportation and communication public capital has been subject to political influences. These influences are in a way moving the balance point in the efficiency and equity trade-off, putting it to a potentially sub-optimal state. The results are highly relevant for Turkey, given that an alleged strong presence of such influences have long been part of political debates.

\subsection{Remedies Provided for the Problems in Turk- ish Regional Data Sets}

For the three studies on Turkish regions, the official data that was initially available were highly fragmented. Currently, if a researcher desires to do a regional level economic research on Turkey using recent official data, she/he cannot use a regional output series with a time span that includes observations from before 2004. Moreover, the researcher will not find any output data for any region for the years 2002 and 2003. For covering the 1990's, the researcher would have to use a gross 
regional product data set that covers the non-recent period of 1987-2001. However, this series would not be comparable to the aforementioned recent series; while the latter is measured as gross regional product, the former is measured as gross regional value added. Moreover, the former is NUTS- $2^{1}$ level data while the latter is in NUTS-3 level. Additionally, a similar problem exists for regional investment data on transportation and communication public investments; they are not recorded by province unless investments are done exclusively only to one province (NUTS-3 level region). Therefore, if a road investment was made to more than one province, the value of this investment would not be recorded under the investment figures for any spatial unit.

In the presence of these difficulties, the spatial units used in the analysis within this thesis were the 26 NUTS-2 level statistical regions of the Republic of Turkey. Using certain methods for managing the fragmented data, the thesis presents a regional gross value added series for Turkey for a relatively long period of time $\left(1987-2011,{ }^{2}\right.$ with the possibility to be extended further as new data is published). As stated earlier, this regional gross value added (GVA) series was previously not available due to gaps in the official data, differences in measurement of regional output in different periods, and differences in the spatial scale of data collection in different periods. The appendices of Chapter 5 elaborate in detail on how these problems were tackled. As a result, a consistent regional GVA series for the period 1987-2011 (and potentially further) is made available to researchers who aim to use regional output data for Turkish regions. The appendices also elaborate on the remedy suggested for the above-mentioned drawback on regional public investment data. To summarize, the techniques used were imputation by the estimation of the missing years' gross value added for each region, consolidation of the figures measured as gross regional product and gross regional value added, aggregation of spatial units, and inflation of figures to account for missing investment values. As a result, the appendices of Chapter 5

\footnotetext{
"Nomenclature of territorial units for statistics," see: http://epp.eurostat.ec.europa.eu/portal/page/portal/nuts _nomenclature/introduction

${ }^{2}$ At the time of the submission of this thesis, the Turkish regional national accounts data existed only for until 2011.
} 
present customizable guidelines for researchers to cope with these data issues regarding the Turkish regional output and public investment series.

Moreover, as earlier stated, a ready-to-use data set for Turkish regions consisting of the densities of various types of infrastructure and their locations is used in Chapter 4. This data set brings together figures that were highly detached for each year and province, and constructs a full NUTS-2 level data set for Turkish regions.

\subsection{Methodological Approaches, Challenges and Their Implications}

The methodological approaches adopted in this thesis were meta-analysis with an emphasis on the possibility of publication bias, dynamic panel and instrumental variable estimations, spatial econometric models, and gravity model estimations where sample selection bias is considered.

Certain limitations exist in relation to the utilized empirical approaches. One limitation that is not specific to this dissertation is related to Chapter 4 where a gravity model was used. The Turkish regional variables were gathered from various sources and their measurements did not correspond to those for world countries found in commonly used global data sets. As a result, it was not possible to construct a full data matrix where all variables for all units of observations could be put together. Therefore, instead of a full bilateral setting where each spatial unit would enter as both importer and exporter, the research strategy resulted in focusing only on Turkish regions as exporters and world countries as importers, and infrastructure-related explanatory variables were included only for the exporting regions. On the other hand, this limitation which is dictated by data unavailability, applies to any research that would aim to do a gravity study using infrastructurerelated variables for Turkish regions.

Another limitation is regarding a trade-off between taking into account 
time dynamics versus spatial effects. This is because, as also mentioned in Chapter 2, a dynamic spatial panel model is still a huge challenge for researchers who are not econometricians with high expertise in spatial models. Software routines for correctly estimating such models are not yet widely available to researchers from other disciplines. While one could "run" such a model and obtain some results using certain statistical packages, the econometric concerns regarding their validity would be many, as the method itself is still under development.

\subsection{Future Research Prospects}

The answers and approaches to the research questions in this dissertation also have certain implications for future research possibilities. The topic of "digital divide," which "refers to the perceived gap between those who have access to the latest information technologies and those who do not" (Compaine, 2001), is subject to heated debates (Van Dijk and Hacker, 2003). As observed in this thesis, internet infrastructure is a relevant policy measure within this context, especially in terms of regional disparities. It is remarkable that the relationship has not attracted empirical attention. While Chapter 2 specifically focuses on this topic, further research would shed more light on how enhancements in internet infrastructure can help regions converge in terms of per-capita income through increasing the speed of the distribution of information and the resulting homogenization of regional economies and cultures. For instance, the effect of internet on convergence can be further solidified by researching the micro level mechanisms such as firm and market connectivities leading to the homogenization of, say, corporate cultures across the firms located in different regions of an economy.

The results observed in the meta-analytic study presented in Chapter 3 suggest that empirical research has persistently found infrastructure improvements to enhance exports of an economy more than its imports. While we elaborate on the reasons why this result may have been observed, it is of importance that further attention is devoted to this 
finding. The utilization of trade-enhancing infrastructure within the context of export/import activities and the significance of improvements in such infrastructures regarding firm behavior can be elaborated further, as this may provide more information on how the same infrastructure may influence export flows and import flows differently, and why this non-symmetric outcome is observed.

Finally, a more in-depth look on political factors that play a role in the allocation decisions of transportation and communication public infrastructure can be done by designing case studies which implement face-to-face interviews or surveys. While this dissertation adopted a purely statistical approach to this question, discussions with stakeholders could shed more light on the topic. 


\section{Valorization $^{1}$}

This addendum to the $\mathrm{PhD}$ thesis, in accordance with article 23 of section "Note on the Regulation Governing the Attainment of Doctoral Degrees" of the document "Regulation governing the attainment of doctoral degrees" of Maastricht University, ${ }^{2}$ aims to address the following five points specified in this document: relevance, target groups, activities/products, innovation, and schedule and implementation. As specified in the aforementioned official document, "the addendum about valorisation does not form part of the dissertation and should not be assessed as part of the dissertation."

\section{Relevance}

The social and economic relevance of this dissertation, in addition to the scientific relevance, can be discussed within several contexts. Firstly, from a public policy making perspective, the dissertation elaborates on the outcomes and determinants of investments in certain types of public infrastructure. For instance, in the second chapter, the impact of internet infrastructure on regional per capita income convergence in an upper-middle income economy was examined. The theoretical base was built on the assumptions that the diffusion of information makes regional economies more similar by homogenizing them in terms of markets, financial services, and culture. In turn, it was recognized that telecommunications infrastructure increases the speed of information transfer

\footnotetext{
${ }^{1}$ This addendum contains text from the introduction and conclusion (chapters 1 and $6)$.

${ }^{2}$ http://www.maastrichtuniversity.nl/web/Main/Research/PostgraduateResearch/ PracticalMatters/Regulation $\backslash$ GoverningTheAttainmentOfDoctoralDegrees.htm.
} 
among regions. Finally, a specific, and modern type of telecommunication infrastructure was taken into focus, namely, internet infrastructure. In this chapter, the core-periphery structure of the Turkish economic geography is also explored.

The spatial units used in the analysis within this chapter were the 26 NUTS-2 level statistical regions ${ }^{3}$ of the Republic of Turkey, an uppermiddle income economy (The World Bank, 2014). The results have supported the hypothesis that better internet infrastructure makes regional economies more similar by increasing their speeds of convergence to their steady-state levels of per capita income, and also by making the individual steady-states of regional economies more alike. As a result, for countries like Turkey, if regional disparities are seen as a problem, increasing the regional communication ties through modern communication technologies is suggested as a relevant policy tool. On the other hand, in terms of scientific relevance, Chapter 2 provided an application of methods of spatial analysis on Turkey. The dissertation in general obtained and used data on Turkish regions that prior to the remedies suggested in the dissertation, were highly fragmented. Extensive use was made using the statistical software STATA 13 in conducting all analyses.

The results of the dissertation also provided relevant results in terms of the policies regarding trade-related infrastructure. A meta-analytic study is presented in Chapter 3 with the purpose of synthesizing previous research results on studies looking at the relationship between infrastructure and trade. An interesting result was observed which also rises policy-relevant questions. This can be summarized as such: previous studies persistently estimated that trade-related infrastructure such as ports, roads, airports, railroads, and communication infrastructures increase the exports of the economy in which they are located more than its imports. This is obviously an outcome that needs further attention. Such a result would suggest from a policy making perspective that ceteris paribus, enhancing own trade related infrastructure could help improve the trade balance in an economy. Why such results have

\footnotetext{
"Nomenclature of territorial units for statistics," see: http://epp.eurostat.ec.europa.eu/portal/page/portal/nuts _nomenclature/introduction
} 
been observed in the literature is further discussed in the concluding section of this chapter. In terms of scientific relevance, this chapter presents the first meta-analytic application on the question of the relationship between infrastructure and trade. The analysis follows the concrete guidelines for conducting a meta-analysis provided in Stanley et al. (2013). The study also considers the multidimensional nature of the concept of "infrastructure" and distinguishes its components.

Regarding the policy relevance of trade-related infrastructure, further evidence is presented in Chapter 4 where a case-specific focus is taken. Using the regions in Turkey, the chapter assesses not only the impact of the presence of infrastructure, but also the effects of the location of point infrastructures (e.g. ports and airports) as exit or entry nodes for trade flows. Results have essential implications on policy decisions regarding where to locate infrastructure and what type of infrastructure is good for exports. The study is the first application of a gravity model on the export flows of Turkish regions, and underlines the importance of private ports, air transport infrastructure, and land infrastructure.

The dissertation does not only look at how infrastructure affects convergence and trade, but also at how it is allocated by the central planners in Turkey. This is especially relevant for regional policy making and its applications. I assume, in line with the dominant theories in the literature, that there are two main points of interest in the decision making process regarding the allocation of public capital: national efficiency and regional equity. The policymakers, depending on their purposes, allocate weights on these goals in various degrees. On the other hand, this mechanism of preference may be influenced by other factors, mainly, political ones. This chapter shows that in Turkey, the regional allocation of investments in transportation and communication public capital has been subject to political influences. These influences are in a way moving the balance point in the efficiency and equity trade-off, putting it to a potentially sub-optimal state. The results are highly relevant for Turkey, given that an alleged strong presence of such influences have long been part of political debates.

Scientifically, the research in Chapter 5 , is the only study that recognizes 
the dependency between allocation decisions of different infrastructure types when looking at the determinants of public investments. Moreover, the study also presents a regional gross value added series for Turkey for a relatively long period of time (1987-2011, with the possibility to be extended further as new data is published). This regional gross value added series was previously not available due to gaps in the official data, differences in measurement of regional output in different periods, and differences in the spatial scale of data collection in different periods. Finally, aside of public policy related implications, the dissertation also is relevant for location choices of firms. Firms will locate to where increasing external returns to scale is higher. Moreover, reduction in transportation and communication costs also highly affect the agglomeration of firms and industries, as formulated in the New Economic Geography literature by Paul Krugman. ${ }^{4}$ Therefore, infrastructure-related policies are important policy tools that partly determine the agglomeration of economic activity over space.

\section{Target Groups}

The target groups of this dissertation are academics, policy-makers and policy-analysts, firms, non-governmental organizations, and political parties. In the academic sense, as mentioned in the previous section, the study presents novelties in terms of analytical contributions, synthesis, applications of various methodologies, and reconciled data sets for the use of future research projects.

For policy-makers and policy-analysts, this dissertation provides information and evidence regarding infrastructure related policies and their outcomes, how they are undertaken, how their location choices should be, and also present suggestions on the separate functions of different types of public infrastructures.

In the context of private firms, the dissertation has implication on

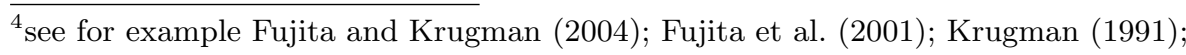
David (1999). 
location choices in relation to infrastructure presence and agglomeration. The dissertation also gives information for firms regarding what benefits to to expect from what infrastructure type.

Finally, for non-governmental organizations and political parties in Turkey, Chapter 5 provides important evidence that allocation decisions of the central government in relation to regional transportation and communication public capital is subject to political influence. This evidence can contribute scientifically to political discussions in need of academic research findings.

\section{Activities/Products}

The results of this dissertation can be translated to activities regarding the enhancement and location choice of infrastructure based on specific regional and national needs. In turn, such activities would influence the general economic geography in a given group of regional economies. Moreover, results can also support political activities that aim towards attaining an optimal strategy in allocating infrastructure across the regions of Turkey.

Further activities that could arise are related to an international dimension of investing in the infrastructures of trade partners. Also, general national policies on trade promotion or export strategies could benefit from the results mentioned earlier. Such activities, of course, are dependent on the general policy goals of the relevant policymakers.

\section{Innovation}

The results presented in this dissertation stem from a collection of innovative components. For instance, recognizing dependencies between capital allocation decisions in Chapter 5, or using an interaction variable approach to see how the speed of convergence is affected by the main 
explanatory variable of interest are innovative contributions. Moreover, focusing on internet infrastructure as a modern mode of communication infrastructure in assessing regional per capita income convergence, providing meta-analytic results on the infrastructure-trade relationship, and special focus on the implications of infrastructure enhancements in the Turkish regions within the last decade provide new approaches and case specific results to the relevant academic strand of literature.

\section{Schedule and Implementation}

The schedule and implementation in relation to the results presented in this $\mathrm{PhD}$ dissertation, are of course entirely dependent on the potential interest of policymakers. Naturally, it is unlikely that states shape whole policies by considering one paper or dissertation on economic policies. It is rather an accumulation of knowledge and empirical results that ultimately impact policy making. It is my hope that this dissertation has contributed numerous novel empirical bits of evidence that will add to this pool of knowledge that eventually translates into policy making in conjunction with the results presented in the literature in general.

\section{Concluding Discussion for the Valorization Addendum}

Connectivity of regional economies in relation to costs of transportation and communication, the agglomeration of industries over space, and the decision mechanisms that affect these processes are key components of the spatial economy. This valorisation addendum to the $\mathrm{PhD}$ dissertation titled "Regional policies, convergence and trade: studies on Turkish regions and a meta-analysis" aimed to address how the findings of the studies composing the thesis is relevant to the economic and social occurrences.

Three our of the four studies represented as separate chapters in the thesis focus on the regional economies of Turkey. Therefore, for similar 
upper-middle income economies, the dissertation presents policy relevant suggestions within the context of regional infrastructural policies. Another study presented in the thesis aims to combine the research findings of many previous research findings on how regional trade performances can be enhanced through policies related to infrastructure.

A diverse collection of methodologies have been used in answering the research questions which where (1) to what extent internet infrastructure reduces regional disparities? (2) to what extent infrastructure impacts on trade flows? and (3) to what extent political influences determine the regional allocation of transportation and communication public infrastructure? These questions were addressed using methodologies such as dynamic panel estimation, meta-analysis, gravity models, and spatial econometrics.

The results, other than academic contributions, also underline certain indirect implications about firm location and economic clustering. For instance, the spatial analyses presented in Chapter 2 point out how regions with similar economic characteristics are clustered over space, implying that firms must be concentrating on certain areas of the country in question. Moreover, as mentioned in the concluding section of Chapter 3, the difference between the estimated exporter infrastructure elasticity of trade and the importer infrastructure elasticity of trade could be due to how exporting firms respond to improvements in own infrastructure versus trade partner infrastructure. The firm location choices also depend on the presence of infrastructure in given areas and the type of such infrastructures together with their distance to economic centers. Finally, the dissertation also suggests that firms in Turkey need to be wary about the political atmosphere in their regions as they can have important implications on how regional infrastructure is supplied and maintained.

Certainly, future research will shed more light on the research questions this dissertation focuses on. For instance, the utilization of infrastructure within the context of trade activities can be elaborated further, as it may provide more information how the same infrastructure may influence export flows and import flows differently. Also, the affect of 
internet on convergence can be further solidified by researching the micro level mechanisms such as firm and market connectivities leading to the homogenization of, say, corporate cultures across the firms located in different regions of an economy. Finally, the lobbying power of regions for attracting more investments from the central government can also be researched using case study approaches, interview based data collection, or survey focused research. 


\section{Bibliography}

Altunc, Ö. F. and Senturk, B. (2010). The role of public and private investment to ensure sustainable macroeconomic stability in Turkey.

Anderson, J. E. and van Wincoop, E. (2003). Gravity with gravitas: A solution to the border puzzle. American Economic Review, 93(1):170192.

Anderson, J. E. and van Wincoop, E. (2004). Trade costs. Journal of Economic Literature, 42(3):691-751.

Anselin, L. (1988). Spatial econometrics: methods and models, volume 4. Springer.

Anselin, L. (1995). Local indicators of spatial association-LISA. Geographical Analysis, 27(2):93-115.

Anselin, L. (1996). The Moran scatterplot as an ESDA tool to assess local instability in spatial association. Spatial analytical perspectives on GIS, 4:111-127.

Anselin, L., Syabri, I., and Kho, Y. (2006). GeoDa: An introduction to spatial data analysis. Geographical Analysis, 38(1):5-22.

Appold, S. J. (1995). Agglomeration, interorganizational networks, and competitive performance in the US metalworking sector. Economic geography, pages 27-54.

Arbia, G. (2006). Spatial econometrics: statistical foundations and applications to regional convergence. Springer. 
Arellano, M. and Bond, S. (1991a). Some tests of specification for panel data: Monte carlo evidence and an application to employment equations. The Review of Economic Studies, 58(2):277-297.

Arellano, M. and Bond, S. (1991b). Some tests of specification for panel data: Monte carlo evidence and an application to employment equations. The Review of Economic Studies, 58(2):277-297.

Arestis, P., Baddeley, M., and McCombie, J. S. (2007). Economic growth: new directions in theory and policy. Edward Elgar Publishing.

Armstrong, H. and Taylor, J. (2000). Regional economics and policy, volume Third edition. Blackwell Publishing.

Aschauer, D. A. (1989a). Does public capital crowd out private capital? Journal of Monetary Economics, 24(2):171-188.

Aschauer, D. A. (1989b). Is public-expenditure productive. Journal of Monetary Economics, 23(2):177-200. ISI Document Delivery No.: U9363 Times Cited: 842 Cited Reference Count: 50 Aschauer, da Elsevier science bv Amsterdam.

Aschauer, D. A. (2000). Do states optimize? public capital and economic growth. The Annals of Regional Science, 34(3):343-363.

Ashenfelter, O., Harmon, C., and Oosterbeek, H. (1999). A review of estimates of the schooling/earnings relationship, with tests for publication bias. Labour Economics, 6(4):453-470.

Australian Business Deans Council (ABDC) (2010). Australian Business Deans Council Journal Quality List. Retrieved on September 24, 2012 from http://www.abdc.edu.au/.

Badinger, H., Müller, W., and Tondl, G. (2004). Regional convergence in the European Union, 1985- 1999: A spatial dynamic panel analysis. Regional Studies, 38(3):241-253.

Baier, S. L. and Bergstrand, J. H. (2009). Bonus vetus ols: A simple method for approximating international trade-cost effects using the gravity equation. Journal of International Economics, 77(1):77 - 85. 
Baltagi, B. H. and Bresson, G. (2011). Maximum likelihood estimation and lagrange multiplier tests for panel seemingly unrelated regressions with spatial lag and spatial errors: An application to hedonic housing prices in Paris. Journal of Urban Economics, 69(1):24-42.

Bandyopadhyay, U. (1999). Trade and the distribution sector: Some evidence from OECD countries. The Canadian Journal of Economics / Revue canadienne d'Economique, 32(5):1299-1312.

Barro, R. J. and Sala-i Martin, X. (1992). Convergence. Journal of political Economy, pages 223-251.

Barro, R. J. and Sala-i-Martin, X. (2003). Economic Growth, 2nd Edition, volume 1 of MIT Press Books. The MIT Press.

Barro, R. J., Sala-i Martin, X., Blanchard, O. J., and Hall, R. E. (1991). Convergence across states and regions. Brookings papers on economic activity, pages 107-182.

Battisti, M. and De Vaio, G. (2008). A spatially filtered mixture of $\beta$-convergence regressions for EU regions, 1980-2002. Empirical Economics, 34(1):105-121.

Becker, B. J. and Wu, M.-J. (2007). The synthesis of regression slopes in meta-analysis. Statistical Science, pages 414-429.

Beckerman, W. (1956). Distance and the pattern of intra-European trade. The Review of Economics and Statistics, 38(1):31-40.

Behrman, J. R. and Craig, S. G. (1987). The distribution of public services: An exploration of local governmental preferences. The American Economic Review, pages 37-49.

Belotti, F., Hughes, G., and Mortari, A. P. (2013). XSMLE: Stata module for spatial panel data models estimation. Statistical Software Components, Boston College Department of Economics.

Berechman, J., Ozmen, D., and Ozbay, K. (2006). Empirical analysis of transportation investment and economic development at state, county and municipality levels. Transportation, 33(6):537-551. 
Bergstrand, J. H. (1985). The gravity equation in international trade: Some microeconomic foundations and empirical evidence. The Review of Economics and Statistics, 67(3):474-481.

Berthelon, M. and Freund, C. (2008). On the conservation of distance in international trade. Journal of International Economics, 75(2):310 -320 .

Bhatta, S. D. and Drennan, M. P. (2003). The economic benefits of public investment in transportation: A review of recent literature. Journal of Planning Education and Research, 22(3):288-296.

Biehl, D. (1986). The contribution of infrastructure to regional development : final report / by Dieter Biehl, Intrastructure Study Group. Office for Official Publications of the European Communities ; European Community Information Service [distributor], Luxembourg : Washington, DC. Vol. 2 has subtitle: Annex.

Bikker, J. A. (1987). An international trade flow model with substitution: an extension of the gravity model. Kyklos, 40(3):315-337.

Blundell, R. and Bond, S. (1998). Initial conditions and moment restrictions in dynamic panel data models. Journal of econometrics, 87(1):115-143.

Bom, P. and Ligthart, J. E. (2008). How productive is public capital? a meta-analysis.

Bouet, A., Mishra, S., and Roy, D. (2008). Does Africa trade less than it should, and if so, why?: the role of market access and domestic factors. International Food Policy Research Institute, Washington DC, USA.

Bougheas, S., Demetriades, P. O., and Morgenroth, E. L. (1999). Infrastructure, transport costs and trade. Journal of International Economics, 47(1):169-189.

Breuer, J. B., Hauk Jr, W., and McDermott, J. (2014). The return of convergence in the US states. Applied Economics Letters, 21(1):64-68.

Bröcker, J. (2002). Spatial effects of European transport policy: a cge approach. In Trade, networks and hierarchies, pages 11-28. Springer. 
Bröcker, J. and Rietveld, P. (2009). 9 infrastructure and regional development. Handbook of Regional Growth and Development Theories, page 152 .

Bruinsma, F., Nijkamp, P., and Rietveld, P. (1989). Employment impacts of infrastructure investments: a case study for the Netherlands. Serie Research MeMoranda 0051, VU University Amsterdam, Faculty of Economics, Business Administration and Econometrics, Amsterdam.

Brun, J.-F., Carrère, C., Guillaumont, P., and De Melo, J. (2005). Has distance died? Evidence from a panel gravity model. The World Bank Economic Review, 19(1):99-120.

Busemeyer, M. R. (2007). Determinants of public education spending in 21 OECD democracies, 1980-2001. Journal of European Public Policy, 14(4):582-610.

Button, K. (1998). Infrastructure investment, endogenous growth and economic convergence. The Annals of Regional Science, 32(1):145-162.

Buys, P., Deichmann, U., and Wheeler, D. (2010). Road network upgrading and overland trade expansion in Sub-Saharan Africa. Journal of African Economies, 19(3):399-432.

Cadot, O., Röller, L., and Stephan, A. (1999). A Political Economy Model of Infrastructure Allocation: An Empirical Assessment. Centre for Economic Policy Research.

Cantos, P., Gumbau Albert, M., and Maudos, J. (2005). Transport infrastructures, spillover effects and regional growth: evidence of the Spanish case. Transport Reviews, 25(1):25-50.

Capello, R. (2009). Space, growth and development. Handbook of regional growth and development theories, page 33 .

Capello, R., Fratesi, U., and Resmini, L. (2011). Globalization and Regional Growth in Europe: Past Trends and Future Scenarios. Springer.

Capello, R. and Nijkamp, P. (2009). Introduction: regional growth and development theories in the twenty-first century-recent theoretical 
advances and future challenges. Handbook of regional growth and development theories, pages 1-18.

Carey, J. W. (2008). Communication as culture, revised edition: Essays on media and society. Routledge.

Carrère, C. (2006). Revisiting the effects of regional trade agreements on trade flows with proper specification of the gravity model. European Economic Review, 50(2):223-247.

Carrère, C. and Schiff, M. (2005). On the Geography of Trade. Distance is Alive and Well. Revue économique, 56(6):1249-1274.

Casas, F. R. (1983). International trade with produced transport services. Oxford Economic Papers, 35(1):89-109.

Castells, A. and Sole-Olle, A. (2005). The regional allocation of infrastructure investment: The role of equity, efficiency and political factors. European Economic Review, 49(5):1165-1205.

Castells, M., Fernandez-Ardevol, M., Qiu, J. L., and Sey, A. (2007). Mobile Communication and Society: a global perspective. The MIT Press.

Cipollina, M. and Pietrovito, F. (2011). Trade impact of EU preferential policies: a meta-analysis of the literature. In De Benedictis, L. and Salvatici, L., editors, The Trade Impact of European Union Preferential Policies: An Analysis Through Gravity Models, pages 91-110. SpringerVerlag, Berlin, Heidelberg.

Cipollina, M. and Salvatici, L. (2010). Reciprocal trade agreements in gravity models: A meta-analysis. Review of International Economics, 18(1):63-80.

Commission for Africa (2005). Report of the Commission for Africa, www. commissionforafrica.info. Technical report, Commission for Africa.

Compaine, B. M. (2001). The digital divide: Facing a crisis or creating a myth? Mit Press. 
Costa-I-Font, J., Rodriguez-Oreggia, E., and Lunapla, D. (2003). Political competition and pork-barrel politics in the allocation of public investment in mexico. Public Choice, 116(1/2):185-204.

Coulibaly, S. and Fontagné, L. (2005). South-south trade: geography matters. Journal of African Economies, 15(2):313-341.

Crain, W. M. and Oakley, L. K. (1995). The politics of infrastructure. Journal of Law and Economics, 38(1):1-17.

David, P. A. (1999). Krugman's economic geography of development: NEGs, POGs, and naked models in space. International Regional Science Review, 22(2):162-172.

De, P. (2007). Empirical estimates of trade costs for Asia. Research and information system for developing countries (RIS), India Habitat Centre, New Delhi 110003.

De Groot, H. L., Poot, J., and Smit, M. J. (2009). Agglomeration externalities, innovation and regional growth: theoretical perspectives and meta-analysis. In Capello, R. and Nijkamp, P., editors, Handbook of Regional Growth and Development Theories, pages 256-281. Edward Elgar Publishing.

De Groot, M. A. H. L. and Florax, R. J. (2005). A meta-analysis of $\beta$-convergence: the legendary 2\%. Journal of Economic Surveys, 19(3):389-420.

Debarsy, N. and Ertur, C. (2010). Testing for spatial autocorrelation in a fixed effects panel data model. Regional Science and Urban Economics, 40(6):453-470.

Del Bo, C., Florio, M., and Manzi, G. (2010). Regional infrastructure and convergence: Growth implications in a spatial framework. Transition Studies Review, 17(3):475-493.

Dettmer, B. (2011). International service transactions: Is time a trade barrier in a connected world? Jena Economic Research Papers 2011003, Friedrich Schiller University Jena, Jena. 
Devlet Planlama Teskilati. Dokuzuncu kalkinma plani 2007-2013, 2009 yili yatirim programi. http://www.kalkinma.gov.tr/Pages/ KamuYatirimProgramlari.aspx.

Devlet Planlama Teskilati. Dokuzuncu kalkinma plani 2007-2013, 2010 yili yatirim programi. http://www.kalkinma.gov.tr/Pages/ KamuYatirimProgramlari.aspx.

Ding, L., Haynes, K., and Liu, Y. (2008). Telecommunications infrastructure and regional income convergence in China: panel data approaches. The Annals of Regional Science, 42(4):843-861.

Disdier, A.-C. and Head, K. (2008). The puzzling persistence of the distance effect on bilateral trade. The Review of Economics and Statistics, 90(1):37-48.

Dobbs, R., Pohl, H., Lin, D.-Y., Mischke, J., Garemo, N., Hexter, J., Matzinger, S., Palter, R., and Nanavatty, R. (2013). Infrastructure productivity: how to save $\$ 1$ trillion a year. Instututional report, McKinsey Global Institute.

Dokmeci, V. and Berkoz, L. (1996). International telecommunications in Turkey. Telecommunications Policy, 20(2):125 - 130.

Donaghy, K. P. (2009). Regional Growth and Trade in the New Economic Geography and Other Recent Theories. Edward Elgar Publishing, Inc., Cheltenham, UK.

Doucouliagos, H. and Laroche, P. (2009). Unions and profits: A metaregression analysis. Industrial Relations: A Journal of Economy and Society, 48(1):146-184.

Drukker, D. M., Peng, H., Prucha, I., and Raciborski, R. (2011). Sppack: Stata module for cross-section spatial-autoregressive models. Statistical Software Components, Boston College Department of Economics.

Duffy-Deno, K. T. and Eberts, R. W. (1991). Public infrastructure and regional economic development: a simultaneous equations approach. Journal of Urban Economics, 30(3):329-343. 
Durlauf, S. N., Johnson, P. A., and Temple, J. R. (2005). Growth econometrics. Handbook of economic growth, 1:555-677.

Egger, M., Smith, G. D., Schneider, M., and Minder, C. (1997). Bias in meta-analysis detected by a simple, graphical test. The $B M J$, 315(7109):629-634.

Egger, P. and Larch, M. (2008). The bilateral and multilateral trade effects of road and railway transport infrastructure. Working paper, Munich, Germany: Ifo Institute for Economic Research, LudwigMaximilian University of Munich.

Egger, P. H. and Lassmann, A. (2012). The language effect in international trade: A meta-analysis. Economics Letters, 116(2):221-224.

Elbadawi, I. (1999). Can Africa Export Manufactures?: The Role of Endowment, Exchange Rates and Transaction Costs, volume 2120. World Bank Publications, Washington DC, USA.

Elbadawi, I., Mengistae, T., and Zeufack, A. (2006). Market access, supplier access, and Africa's manufactured exports: A firm level analysis. The Journal of International Trade $\&$ Economic Development, 15(4):493-523.

Elhorst, J. P. (2003). Specification and estimation of spatial panel data models. International regional science review, 26(3):244-268.

Elhorst, J. P. (2010). Applied spatial econometrics: Raising the bar. Spatial Economic Analysis, 5(1):9-28.

Elhorst, J. P. (2012). Dynamic spatial panels: models, methods, and inferences. Journal of Geographical Systems, 14(1):5-28.

Elhorst, J. P. and Freret, S. (2009). Evidence of political yardstick competition in France using a two-regime spatial durbin model with fixed effects*. Journal of Regional Science, 49(5):931-951.

Elhorst, P., Piras, G., and Arbia, G. (2010). Growth and convergence in a multiregional model with space-time dynamics. Geographical Analysis, 42(3):338-355. 
Engels, E. A., Schmid, C. H., Terrin, N., Olkin, I., and Lau, J. (2000). Heterogeneity and statistical significance in meta-analysis: an empirical study of 125 meta-analyses. Statistics in Medicine, 19(13):17071728 .

Ersoy, A. (2014). Turkey's protean regional policy: Does it happen on the ground? Middle Eastern Studies, (ahead-of-print):1-20.

Ertugal, E. and Dobre, A. M. (2011). Dynamics of regionalisation and the impact of the EU: Comparing regional reforms in romania and Turkey. Europe-Asia Studies, 63(7):1195-1222.

Ertur, C. and Koch, W. (2007). Growth, technological interdependence and spatial externalities: theory and evidence. Journal of Applied Econometrics, 22(6):1033-1062.

Ertur, C. and Musolesi, A. (2012). Spatial autoregressive spillovers vs unobserved common factors models. a panel data analysis of international technology diffusion. Technical report, INRA UMR CESAER, Centre d'Economie et Sociologie appliquées à l'Agriculture et aux Espaces Ruraux.

Fernald, J. G. (1999). Roads to prosperity? assessing the link between public capital and productivity. The American Economic Review, 89(3):pp. 619-638.

Filiztekin, A. (2008). Türkiye'de bölgesel farklar ve politikalar. TUSIAD.

Florax, R. J., de Groot, H. L., and De Mooij, R. A. (2002). Metaanalysis: A tool for upgrading inputs of macroeconomic policy models. Tinbergen Institute Discussion Paper 02-041/3, Tinbergen Institute, 1082 MS Amsterdam, the Netherlands.

Florax, R. J. and Van der Vlist, A. J. (2003). Spatial econometric data analysis: Moving beyond traditional models. International Regional Science Review, 26(3):223-243.

Forman, C., Goldfarb, A., and Greenstein, S. (2009). The internet and local wages: Convergence or divergence? Technical report, National Bureau of Economic Research. 
Forman, C., Goldfarb, A., and Greenstein, S. (2012). The internet and local wages: A puzzle. American Economic Review, 102(1):556.

Francois, J. and Manchin, M. (2007). Institutions, infrastructure, and trade. Policy Research Working Paper Series 4152, The World Bank, Washington DC, USA.

Fujimura, M. and Edmonds, C. (2006). Impact of cross-border transport infrastructure on trade and investment in the GMS. Asian Development Bank Institute Discussion Paper 48, Asian Development Bank Institute, Tokyo 100-6008, Japan.

Fujita, M. and Krugman, P. (2004). The new economic geography: Past, present and the future*. Papers in regional science, 83(1):139-164.

Fujita, M., Krugman, P., and Venables, A. J. (1999). The Spatial Economy: Cities, Regions and International Trade. MIT Press, Cambridge, MA, London.

Fujita, M., Krugman, P. R., and Venables, A. J. (2001). The spatial economy: Cities, regions, and international trade. MIT press.

Garcia-Mila, T. and McGuire, T. J. (1992). The contribution of publicly provided inputs to states' economies. Regional Science and Urban Economics, 22(2):229-241.

Genc, M., Gheasi, M., Nijkamp, P., and Poot, J. (2012). The impact of immigration on international trade: a meta-analysis. In Nijkamp, P., Poot, J., and Sahin, M., editors, Migration Impact Assessment: New Horizons, chapter 9, pages 301-337. Edward Elgar Publishing, Northampton, Massachusetts 01060.

Geraci, V. J. and Prewo, W. (1977). Bilateral trade flows and transport costs. The Review of Economics and Statistics, 59(1):67-74.

Gezici, F. and Hewings, G. J. (2004). Regional convergence and the economic performance of peripheral areas in Turkey. Review of Urban 83 Regional Development Studies, 16(2):113-132.

Gezici, F. and Hewings, G. J. (2007). Spatial analysis of regional inequalities in Turkey. European Planning Studies, 15(3):383-403. 
Glass, G. V. (1976). Primary, secondary, and meta-analysis of research. Educational Researcher, 5(10):3-8.

Glomm, G. and Ravikumar, B. (1994). Public investment in infrastructure in a simple growth model. Journal of Economic Dynamics and Control, 18(6):1173 - 1187.

Goddard, J. (1992). Geography of the UK information economy. In Robins, K., editor, Understanding Information: Business, technology and geography. Belhaven Press, London and New York.

Gokyurt, F. (2010). Kamu yatırımlarının programlama izleme sürecine yerelin katılımı. Başbakanlık Devlet Planlama Teşkilatı.

Granato, M. F. (2008). Regional export performance: first nature, agglomeration... and destiny? the role of infrastructure. Research Project supported through funds of the Andean Development Corporation.

Grigoriou, C. (2007). Landlockedness, infrastructure and trade: new estimates for central Asian countries. Policy Research Working Paper Series 4335, The World Bank, Washington DC, USA.

Hamalainen, P. and Malinen, T. (2011). The relationship between regional value-added and public capital in Finland: what do the new panel econometric techniques tell us? Empirical Economics, 40(1):237-252.

Hansen, N. M. (1965). The structure and determinants of local public investment expenditures. The Review of Economics and Statistics, 47(2):150-162.

Harbord, R. M. and Higgins, J. P. T. (2008). Meta-regression in Stata. Stata Journal, 8(4):493-519.

Heckman, J. J. (1979). Sample selection bias as a specification error. Econometrica: Journal of the econometric society, pages 153-161.

Hedges, L. V. et al. (1992). Modeling publication selection effects in meta-analysis. Statistical Science, 7(2):246-255. 
Helpman, E., Melitz, M., and Rubinstein, Y. (2008). Estimating trade flows: Trading partners and trading volumes. The Quarterly Journal of Economics, 123(2):pp. 441-487.

Hernandez, J. and Taningco, A. B. (2010). Behind-the-border determinants of bilateral trade flows in East Asia. Working paper 80, Asia-Pacific Research and Training Network on Trade (ARTNeT).

Heston, A., Summers, R., and Aten, B. (2012). Penn world table version 7.1. Center for International ComParisons of Production, Income and Prices at the University of Pennsylvania.

Hirsch, W. Z. (1959). Expenditure implications of metropolitan growth and consolidation. The Review of Economics and Statistics, 41(3):232241.

Holmgren, J. (2007). Meta-analysis of public transport demand. Transportation Research Part A: Policy and Practice, 41(10):1021-1035.

Holtz-Eakin, D. and Lovely, M. E. (1996). Scale economies, returns to variety, and the productivity of public infrastructure. Regional Science and Urban Economics, 26(2):105-123.

Hoover, E. M. and Giarratani, F. (1971). An introduction to regional economics.

Islam, N. (1995). Growth Empirics: A Panel Data Approach. The Quarterly Journal of Economics, 110(4):1127-70.

Islam, N. (2003). What have we learnt from the convergence debate? Journal of economic surveys, 17(3):309-362.

Iwanow, T. and Kirkpatrick, C. (2007). Trade facilitation, regulatory quality and export performance. Journal of International Development, 19(6):735-753.

Iwanow, T. and Kirkpatrick, C. (2009). Trade facilitation and manufactured exports: Is Africa different? World Development, 37(6):10391050. 
Jansen, M. and Nordås, H. K. (2004). Institutions, trade policy and trade flows. Centre for Economic Policy Research, London EC1V 3PZ, UK.

Joanis, M. (2011). The road to power: partisan loyalty and the centralized provision of local infrastructure. Public Choice, 146(1-2):117-143.

Johansson, B., Karlsson, C., and Stough, R. (2001). Theories of Endogenous Regional Growth - Lessons for Regional Policies. Springer.

Kalkinma Bakanligi. http://www.kalkinma.gov.tr/Pages/ TemelEkonomikGostergeler.aspx.

Karacadag Development Agency (2011). Sanlıurfa sanayisinin yeniden yapılandırılması icin teknik destek projesi - lojistik sektör analiz raporu. Industry report TR 06 02.08-02/001, Karacadag Development Agency Sanlıurfa Investment Support Office.

Karlsson, C. and Olsson, M. (2006). The identification of functional regions: theory, methods, and applications. The annals of regional science, 40(1):1-18.

Kelejian, H. H. and Prucha, I. R. (1998). A generalized spatial two-stage least squares procedure for estimating a spatial autoregressive model with autoregressive disturbances. The Journal of Real Estate Finance and Economics, 17(1):99-121.

Kemmerling, A. and Bodenstein, T. (2006). Partisan politics in regional redistribution do parties affect the distribution of EU structural funds across regions? European Union Politics, 7(3):373-392.

Kemmerling, A. and Stephan, A. (2008). The politico-economic determinants and productivity effects of regional transport investment in Europe. EIB Papers, 13(2):36-60.

Keskin, H. and Sungur, O. (2010). Bolgesel politika ekseninde yasanan donusum: Turkiye'de kalkınma planlarında bolgesel politikaların degisimi. Sosyal BiLIMLer Dergisi, (21):271-293. 
Kirdar, M. and Saracoglu, S. (2007). Migration and regional convergence: an empirical investigation for Turkey. Working paper 2648, Munich personal RePEc archive.

Krugman, P. R. (1991). Geography and trade. MIT press.

Kurmanalieva, E. and Parpiev, Z. (2008). Geography and trade in Central Asia. Technical report, EERC Research Grant Paper RO6-085.

Kustepeli, Y., Gulcan, Y., and Akgungor, S. (2012). Transportation infrastructure investment, growth and international trade in Turkey. Applied Economics, 44(20):2619-2629.

Lagendijk, A., Kayasu, S., and Yasar, S. (2009). The role of regional development agencies in Turkey: From implementing EU directives to supporting regional business communities? European Urban and Regional Studies, 16(4):383-396.

Lakshmanan, T., Nijkamp, P., Rietveld, P., and Verhoef, E. T. (2001). Benefits and costs of transport. Papers in Regional Science, 80(2):139164.

Lall, S. V. and Yilmaz, S. (2001). Regional economic convergence: Do policy instruments make a difference? The annals of regional science, 35(1):153-166.

Lawless, M. (2010). Destinations of Irish exports: A gravity model approach. Journal of the Statistical \& Social Inquiry Society of Ireland, 39:1-22.

Le Gallo, J. and Ertur, C. (2003). Exploratory spatial data analysis of the distribution of regional per capita gdp in Europe, 1980- 1995. Papers in regional science, 82(2):175-201.

Lee, L. and Yu, J. (2010a). Estimation of spatial autoregressive panel data models with fixed effects. Journal of Econometrics, 154(2):165 185 .

Lee, L.-f. and Yu, J. (2010b). Some recent developments in spatial panel data models. Regional Science and Urban Economics, 40(5):255-271. 
Leon-Gonzalez, R. and Montolio, D. (2004). Growth, convergence and public investment. a bayesian model averaging approach. Applied Economics, 36(17):1925-1936.

León-González, R. and Montolio, D. (2004). Growth, convergence and public investment. a bayesian model averaging approach. Applied Economics, 36(17):1925-1936.

LeSage, J. and Pace, R. K. (2009). Introduction to spatial econometrics. Boca Raton, FL: Chapman EHall/CRC.

Limao, N. and Venables, A. J. (2001). Infrastructure, geographical disadvantage, transport costs, and trade. The World Bank Economic Review, 15(3):451-479.

Linders, G.-J. M. (2005). Distance Decay in International Trade Patterns - a Meta-analysis. ERSA conference papers ersa05p679, European Regional Science Association.

Longo, R. and Sekkat, K. (2004). Economic obstacles to expanding intra-African trade. World Development, 32(8):1309-1321.

Maignan, C., Ottaviano, G., and Pinelli, D. (2003). ICT, clusters and regional cohesion: A summary of theoretical and empirical research.

Mankiw, N. G., Romer, D., and Weil, D. N. (1992). A contribution to the empirics of economic growth. The Quarterly Journal of Economics, 107(2):pp. 407-437.

Marin, D. (1992). Is the export-led growth hypothesis valid for industrialized countries? The Review of Economics and Statistics, pages 678-688.

Marquez-Ramos, L. and Martinez-Zarzoso, I. (2005). Does heterogeneity matter in the context of the gravity model? Economics Bulletin, $6(17): 1-7$.

Martin, P. and Rogers, C. A. (1995). Industrial location and public infrastructure. Journal of International Economics, 39(3-4):335-351. 
Martincus, C. V., de Carballo, J., and Cusolito, A. (2012). Routes, exports, and employment in developing countries: following the trace of the Inca roads. Preliminary paper.

Martínez-Zarzoso, I. and Nowak-Lehmann, F. (2003). Augmented gravity model: An empirical application to MERCOSUR-European Union trade flows. Journal of applied economics, 6(2):291-316.

Melo, P. C., Graham, D. J., and Noland, R. B. (2009). A meta-analysis of estimates of urban agglomeration economies. Regional Science and Urban Economics, 39(3):332-342.

Mizutani, F. and Tanaka, T. (2008). Productivity effects and determinants of public infrastructure investment. The Annals of Regional Science, 44(3):493-521.

Monfort, P. (2008). Convergence of EU regions: measures and evolution. European Commission, Regional Policy.

Montolio, D. and Sole-Olle, A. (2009). Road investment and regional productivity growth: the effects of vehicle intensity and congestion. Papers in Regional Science, 88(1):99-118.

Moran, P. A. (1950). Notes on continuous stochastic phenomena. Biometrika, 37(1-2):17-23.

Moré, A. E. and Ollé, A. S. (2005). Does decentralization improve the efficiency in the allocation of public investment? evidence from Spain. Documents de treball IEB, (5):1.

Mundell, R. A. (1957). International trade and factor mobility. The American Economic Review, 47(3):321-335.

Munnell, A. H. (1990). Why has productivity growth declined? productivity and public investment. New England Economic Review, 30:3-22.

Munnell, A. H. and Cook, L. M. (1990). How does public infrastructure affect regional economic performance? In Is There a Shortfall in Public Capital Investment? Proceedings of a Conference. 
Nickell, S. (1981). Biases in dynamic models with fixed effects. Econometrica: Journal of the Econometric Society, pages 1417-1426.

Nicoletti, G., Golub, S., Hajkova, D., Mirza, D., and Yoo, K.-Y. (2003). Policies and international integration: influences on trade and foreign direct investment. OECD Economics Department Working Papers 359, OECD Publishing, OECD France.

Nijkamp, P. (1986). Infrastructure and regional development: a multidimensional policy analysis. Empirical Economics, 11(1):1-21.

Nijkamp, P. (2000). Infrastructure and suprastructure in regional competition: A deus ex maChina? Springer.

Nijkamp, P., Gheasi, M., and Rietveld, P. (2011). Migrants and international economic linkages: A meta-overview. Spatial Economic Analysis, 6(4):359-376.

Nijkamp, P. and Poot, J. (2004). Meta-analysis of the effect of fiscal policies on long-run growth. European Journal of Political Economy, 20(1):91-124.

Ninkovic, J. (2009). What determines export success in labor-intensive goods. In Annual meeting of the ISA's 50th Annual Convention on "Exploring the Past, Anticipating the Future" held in New York City, volume 15.

Njinkeu, D., Wilson, J. S., and Powo Fosso, B. (2008). Expanding trade within Africa the impact of trade facilitation. World Bank, Washington DC, USA.

Nordas, H. K. and Piermartini, R. (2004). Infrastructure and trade. WTO Staff Working Paper ERSD-2004-04, Economic Research and Statistics Division WTO.

OECD/WTO (2009). Aid for trade at a glance 2009.

Onder, A. O., Deliktas, E., and Karadag, M. (2010). The impact of public capital stock on regional convergence in Turkey. European Planning Studies, 18(7):1041-1055. 
Onder, Z. and Ozyildirim, S. (2011). Political connection, bank credits and growth: evidence from Turkey. The World Economy, 34(6):10421065 .

Owyang, M. T., Ramey, V. A., and Zubairy, S. (2013). Are government spending multipliers greater during periods of slack? Evidence from twentieth-century historical data. The American Economic Review, 103(3):129-134.

Ozgen, C., Nijkamp, P., and Poot, J. (2010). The effect of migration on income growth and convergence: Meta-analytic evidence. Papers in Regional Science, 89(3):537-561.

Painter, G. and Bae, K.-H. (2001). The changing determinants of state expenditure in the united states: 1965-1992. Public Finance and Management, 9(4):370-392.

Persky, J., Soot, S., Sriraj, P., et al. (2013). Costs and benefits of employment transportation for low-wage workers: An assessment of job access public transportation services. Evaluation and program planning, 37:31-42.

Persson, J. (1997). Convergence across the Swedish counties, 1911-1993. European Economic Review, 41(9):1835 - 1852.

Persson, M. (2007). Trade facilitation and the EU-ACP economic partnership agreements. Conference paper, 10th annual conference on global economic analysis.

Peters, J. L., Sutton, A. J., Jones, D. R., Abrams, K. R., Rushton, L., and Moreno, S. G. (2010). Assessing publication bias in meta-analyses in the presence of between-study heterogeneity. Journal of the Royal Statistical Society: Series A (Statistics in Society), 173(3):575-591.

Pfaffermayr, M. (2012). Spatial convergence of regions revisited: a spatial maximum likelihood panel aapproach. Journal of Regional Science, 52(5):857-873.

Picard, R. and Stepner, M. (2012). MERGEPOLY: Stata module to merge adjacent polygons from a shapefile. Statistical Software Components, Boston College Department of Economics. 
Pisati, M. (2007). SPMAP: Stata module to visualize spatial data. Statistical Software Components, Boston College Department of Economics.

Pisati, M. (2012). Exploratory spatial data analysis using Stata. German Stata Users' Group Meetings 2012 07, Stata Users Group.

Poot, J. (2014). Meta-analysis of previous empirical research findings. In Stimson, R. J., editor, Handbook of Research Methods and Applications in Spatially Integrated Social Science. Cheltenham UK: Edward Elgar.

Portugal-Perez, A. and Wilson, J. S. (2012a). Export performance and trade facilitation reform: hard and soft infrastructure. World Development, 40(7):1295-1307.

Portugal-Perez, A. and Wilson, J. S. (2012b). Export performance and trade facilitation reform: Hard and soft infrastructure. World Development, 40(7):1295-1307.

Puhani, P. (2000). The heckman correction for sample selection and its critique. Journal of Economic Surveys, 14(1):53-68.

Raballand, G. (2003). Determinants of the negative impact of being landlocked on trade: An empirical investigation through the Central Asian case. Comparative Economic Studies, 45(4):520-536.

Ramcharan, R. (2009). Why an economic core: domestic transport costs. Journal of Economic Geography, 9(4):559-581.

Randolph, S., Bogetic, Z., and Hefley, D. (1999). Determinants of public expenditure on infrastructure: Transportation and communication. Availability Note: Information provided in collaboration with the RePEc Project: http://repec.org Accession Number: 0747859; Keywords: Infrastructure; Publication Type: Working Paper; Update Code: 200410.

Ridhwan, M. M., De Groot, H. L., and Nijkamp, P. (2010). The impact of monetary policy on economic activity: Evidence from a meta-analysis. Discussion Paper 10-043/3, Tinbergen Institute, 1082 MS Amsterdam, the Netherlands. 
Robins, K. and Gillespie, A. (1992). Communication, organization and territory. In Robins, K., editor, Understanding Information: Business, technology and geography. Belhaven Press, London and New York.

Romp, W. and De Haan, J. (2007). Public capital and economic growth: A critical survey. Perspektiven der Wirtschaftspolitik, 8(S1):6-52.

Roodman, D. (2009). How to do XTABOND2: An introduction to difference and system GMM in stata. Stata Journal, 9(1):86-136.

Rose, A. K. and Stanley, T. D. (2005). A meta-analysis of the effect of common currencies on international trade. Journal of Economic Surveys, 19(3):347-365.

Sala-i Martin, X. X. (1996a). The classical approach to convergence analysis. The Economic Journal, 106(437):pp. 1019-1036.

Sala-i Martin, X. X. (1996b). Regional cohesion: evidence and theories of regional growth and convergence. European Economic Review, 40(6):1325-1352.

Samuelson, P. A. (1952). The transfer problem and transport costs: The terms of trade when impediments are absent. The Economic Journal, 62(246):278-304.

Samuelson, P. A. (1954). The transfer problem and transport costs, ii: Analysis of effects of trade impediments. The Economic Journal, 64(254):264-289.

Schaffer, M. E. (2005). XTIVREG2: Stata module to perform extended IV/2SLS, GMM and AC/2HAC, LIML and k-class regression for panel data models. Statistical Software Components, Boston College Department of Economics.

Shadish, W. R. and Haddock, C. K. (1994). Combining estimates of effect size. In Cooper, H., Hedges, L. V., and Valentine, J. C., editors, The handbook of research synthesis. Russel Sage Foundation, New York 10065, USA. 
Shehata, E. A. E. (2011). SPAUTOREG: Stata module to estimate Spatial (Lag-Error-Durbin-SAC-SPGKS-SPGSAR-GS2SLS-GS3SLSSPML-SPGS-SPIVREG-IVTobit). Statistical Software Components, Boston College Department of Economics.

Shepherd, B. (2012). The gravity model of international trade: A user guide. Manual by UNESCAP and ARTNeT Gravity Modeling Initiative, United Nations - Economic and Social Commission for Asia and the Pacific, (ST/ESCAP/2645).

Shepherd, B. and Wilson, J. S. (2006). Road infrastructure in Europe and Central Asia: does network quality affect trade? World Bank Policy Research Working Paper 4104, The World Bank, Washington DC, USA.

Sole-Olle, A. (2011). Inter-regional redistribution through infrastructure investment: tactical or programmatic? Public Choice.

Sole-Olle, A. and Sorribas-Navarro, P. (2008). The effects of partisan alignment on the allocation of intergovernmental transfers. differencesin-differences estimates for Spain. Journal of Public Economics, 92(12):2302-2319.

Solow, R. M. (1956). A contribution to the theory of economic growth. The Quarterly Journal of Economics, 70(1):65-94.

Stanley, T. D. (2005). Beyond publication bias. Journal of Economic Surveys, 19(3):309-345.

Stanley, T. D., Doucouliagos, C., and Jarrell, S. B. (2008). Metaregression analysis as the socio-economics of economics research. The Journal of Socio-Economics, 37(1):276-292.

Stanley, T. D., Doucouliagos, H., Giles, M., Heckemeyer, J. H., Johnston, R. J., Laroche, P., Nelson, J. P., Paldam, M., Poot, J., Pugh, G., et al. (2013). Meta-analysis of economics research reporting guidelines. Journal of Economic Surveys, 27(2):390-394.

Stanley, T. D. and Jarrell, S. B. (1989). Meta-regression analysis: A quantitative method of literature surveys. Journal of Economic Surveys, 19(3):299-308. 
StataCorp, LP (2013). Stata data analysis and statistical software. Release 13, College Station, TX, USA.

Stephan, A. (2001). Regional infrastructure policy and its impact on productivity: A comParison of Germany and France. Technical report, Discussion papers//WZB, Wissenschaftszentrum Berlin für Sozialforschung, Forschungsschwerpunkt Markt und Politische Ökonomie.

Suda, M. (1997). Office and plant location with transport costs of information. Journal of Regional Science, 37(1):23-34.

Swan, T. W. (1956). Economic growth and capital accumulation. Economic Record, 32(2):334-361.

The World Bank (2013). Gross domestic product 2013. Retreived November 30, 2014 from http://databank.worldbank.org/data/download/GDP.pdf.

The World Bank (2014). Country and lending groups. Retreived June 2, 2014 from http://data.worldbank.org/about/countryclassifications/country-and-lending-groups.

Tranos, E. (2012). The causal effect of the internet infrastructure on the economic development of European city regions. Spatial Economic Analysis, 7(3):319-337.

Tranos, E. and Gillespie, A. (2009). The spatial distribution of internet backbone networks in Europe a metropolitan knowledge economy perspective. European urban and regional studies, 16(4):423-437.

UN Economic Commission for Africa (UNECA) (2013). Supply-anddemand constraints to intra-Africa trade. In Assessing regional integration in Africa IV, chapter 9. Addis Ababa, Ethiopia.

van Dijk, J., Folmer, H., and Oosterhaven, J. (2009). Regional policy: rationale, foundations and measurement of its effects. Handbook of regional growth and development theories, pages 461-478.

Van Dijk, J. and Hacker, K. (2003). The digital divide as a complex and dynamic phenomenon. The information society, 19(4):315-326. 
Varga, A. (1998). University research and regional innovation: a spatial econometric analysis of academic technology transfers, volume 13. Springer.

Vijil, M. and Wagner, L. (2012). Does aid for trade enhance export performance? investigating the infrastructure channel. The World Economy, 35(7):838-868.

Volpe Martincus, C., Carballo, J., Garcia, P. M., and Graziano, A. (2014). How do transport costs affect firms' exports? Evidence from a vanishing bridge. Economics Letters, 123(2):149-153.

von Thünen, J. (1826). Der isolierte Staat in Beziehung auf Landwirtschaft und Nationalökonomie. Gustav Fischer, Jena.

Ward, M. D. and Gleditsch, K. S. (2008). Spatial regression models, volume 155. Sage.

Wilson, J. S., Mann, C. L., and Otsuki, T. (2004). Assessing the potential benefit of trade facilitation : A global perspective. Policy Research Working Paper Series 3224, The World Bank, Washington DC, USA.

Wu, Y. (2007). Export performance in China's regional economies. Applied Economics, 39(10):1283-1293.

Yamano, N. and Ohkawara, T. (2000). The regional allocation of public investment: Efficiency or equity? Journal of Regional Science, 40(2):205-229.

Yildirim, J., Ocal, N., and Ozyildirim, S. (2009). Income inequality and economic convergence in Turkey: A spatial effect analysis. International Regional Science Review, 32(2):221-254.

Young, A. T., Higgins, M. J., and Levy, D. (2008). Sigma convergence versus beta convergence: Evidence from US county-level data. Journal of Money, Credit and Banking, 40(5):1083-1093.

Yu, Y., Zhang, L., Li, F., and Zheng, X. (2011). On the determinants of public infrastructure spending in Chinese cities: A spatial econometric perspective. The Social Science Journal, 48(3):458-467. 
Zheng, X., Li, F., Song, S., and Yu, Y. (2013). Central government's infrastructure investment across Chinese regions: A dynamic spatial panel data approach. China Economic Review. 


\section{Biography}

After graduating from The Koç School - one of Turkey's most selective high schools - Güney Celbiş attended the bilingual program in Banking and Finance at Bilkent University. Upon obtaining his BA degree in 2002, he earned an MBA in global business from Johnson \& Wales University at Providence, Rhode Island. Güney completed his mandatory military service in 2005 and returned to the United States to study in the MA program in Economics of the University of South Carolina which he completed by writing his thesis titled "The Impact of Economic Activity on Life Expectancy." A member of the Omicron Delta Epsilon Honor Society in Economics, Güney was a PhD student in Economics at North Carolina State University where he completed the core doctoral courses. In UNU-MERIT/MGSoG where he began his $\mathrm{PhD}$ in 2009, he focused on the topics of regional economics, trade, innovation, and political economy in a spatial context. Four of the five articles he authored are collected in his doctoral thesis. 